\title{
Catalytic Cascade Cyclization and Regioselective Hydroheteroarylation of Unactivated Alkenes
}

\author{
Rahul K. Shukla, Atul K. Chaturvedi, Chandra M. R. Volla* \\ Department of Chemistry, Indian Institute of Technology Bombay \\ Powai, Mumbai-400076, India. \\ E-mail: chandra.volla@chem.iitb.ac.in
}

\section{Supporting Information}

Table of Contents

1. General considerations and reagent information

2. General procedure for the synthesis of starting materials

3. Optimization details

4. General synthetic procedures

5. Gram scale synthesis and removal of direction group.

6. Control experiment.

7. Spectroscopic data for new compounds

S16

8. References

S52

9. ${ }^{1} \mathrm{H}$ and ${ }^{13} \mathrm{C}$ NMR spectra of the compounds 
1) General considerations reagent information. Unless otherwise stated, all reactions were carried out under air atmosphere in screw cap reaction tubes. All the solvents were bought from Aldrich in sure-seal bottle and were used as received. Palladium acetate and another reagent was bought from Aldrich. For column chromatography, silica gel (100-200 mesh) from Finar Co. was used. A gradient elution using petroleum ether and ethyl acetate was performed based on Merck aluminum TLC sheets (silica gel 60F254). All directing group attach alkenamide were prepared according to the reported procedures in the literatures and the analytical data are in accord with the literature. ${ }^{1}$

Analytical information. All isolated compounds are characterized by ${ }^{1} \mathrm{H}$ NMR, ${ }^{13} \mathrm{C}$ NMR spectroscopy. In addition, all the compounds are further characterized by HRMS. Copies of ${ }^{1} \mathrm{H}$ NMR and ${ }^{13} \mathrm{C}$ NMR can be found in the supporting information. Nuclear magnetic resonance spectra were recorded either on a Bruker 500 or a $400 \mathrm{MHz}$ instrument. All ${ }^{1} \mathrm{H}$ NMR experiments are reported in units, parts per million (ppm), and were measured relative to the signals for residual chloroform $(7.26 \mathrm{ppm})$ in the deuterated solvent, unless otherwise stated. All ${ }^{13} \mathrm{C}$ NMR spectra was reported in ppm relative to deuteron chloroform $(77.16$ ppm), unless otherwise stated, and all were obtained with ${ }^{1} \mathrm{H}$ decoupling.

\section{2) General procedure for the synthesis of starting materials.}

a) Synthesis of $N$-(quinolin-8-yl)but-3-enamide (1a): Vinyl acetic acid (1.12 mL, $13 \mathrm{mmol}$ ) was charged into a $250 \mathrm{~mL}$ RB flask containing $30 \mathrm{~mL}$ DCM. 8-Aminoquinoline (1.44 g, 10 mmol), collidine (2.6 mL, $20 \mathrm{mmol}$ ), and HATU (4.94 g, $13 \mathrm{mmol}$ ) were added sequentially, and the reaction was stirred at ambient temperature for $16 \mathrm{~h}$. The deep brown solution was diluted with EtOAc $(200 \mathrm{~mL})$, washed with sat. $\mathrm{NaHCO}_{3}(100 \mathrm{~mL} \times 2)$ and brine $(100 \mathrm{~mL} \times 1)$, and purified by column chromatography (10-15\% EtOAc in Hexanes) to afford $1.73 \mathrm{~g}$ (82\%) yield of 1a as a yellow oil. Collidine was found to co-elute during column chromatography and was removed by repeated co-evaporation with large volumes of toluene with care to keep the temperature $\leq 35{ }^{\circ} \mathrm{C}$. Elevated temperatures may lead to isomerization to the conjugated alkene. $^{1}$

b) Synthesis of $N$-protected 2-alkynylbenzenamine.

\section{Step-1 ${ }^{\text {st }}$}




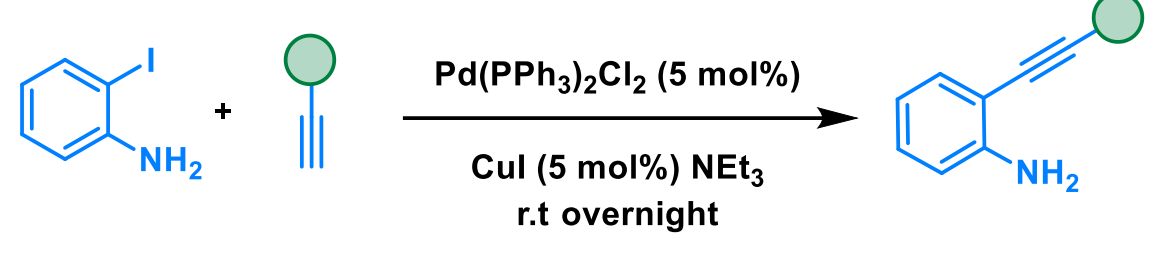

Adapting a known procedure, $\mathrm{CuI}(43.5 \mathrm{mg}, 0.23 \mathrm{mmol}, 5 \mathrm{~mol} \%), \mathrm{PdCl}_{2}\left(\mathrm{PPh}_{3}\right)_{2}(160.2 \mathrm{mg}$, $0.23 \mathrm{mmol}, 5 \mathrm{~mol} \%$ ) and alkyne (5.70 mmol, 1.25 equiv.) were added to a stirred solution of 2-iodoaniline (1.00 g, $4.566 \mathrm{mmol}, 1.0$ equiv.) in $\mathrm{Et}_{3} \mathrm{~N}$ (12.7 mL, $91.32 \mathrm{mmol}, 20$ equiv.), at room temperature. The mixture was allowed to react for $18 \mathrm{~h}$, concentrated under reduced pressure, and submitted to flash Colum chromatography on silica gel to afford the compound.

\section{Step-2 $2^{\text {nd }}$}

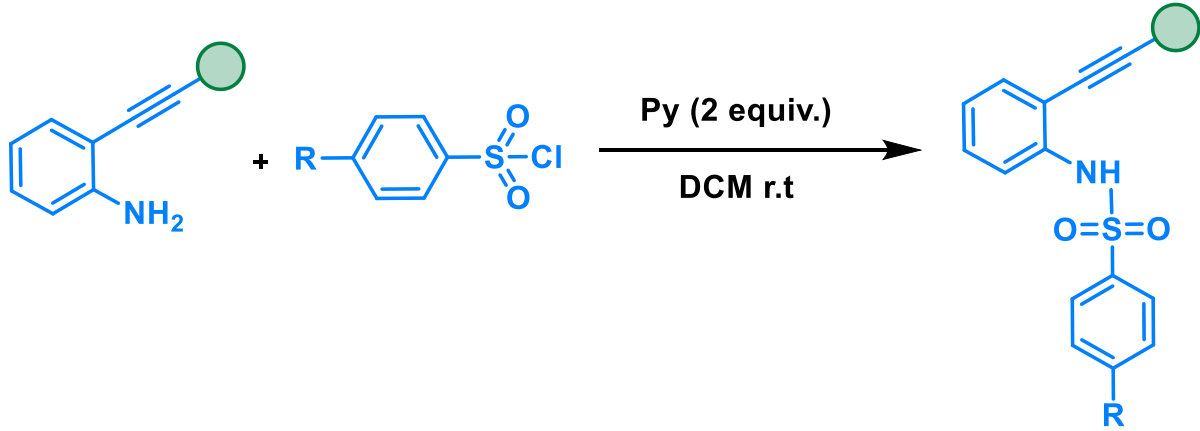

To a solution of 2-(ethynyl)benzenamine (1 equiv.) and pyridine (2 equiv.) in $20 \mathrm{~mL}$ of dichloromethane was added 4-methylbenzene-1-sulfonyl chloride or substituted benzene-1sulfonyl chloride ( 1 equiv.) at $0{ }^{\circ} \mathrm{C}$, then the reaction solution was warmed to room temperature. After the reaction was complete (about $10 \mathrm{~h}$ ) as monitored by TLC, water was added, and the mixture was extracted with ether. The combined organic phase was washed with brine, dried over anhydrous $\mathrm{Na}_{2} \mathrm{SO}_{4}$. The solvent was evaporated under the reduced pressure and the residue was purified by chromatography on silica gel to afford the compound 2. The spectroscopic data of unknown starting materials are given below.

Spectroscopic data for unknown $N$-protected 2-alkynylbenzenamine:-<smiles>CCCCCNc1ccccc1C#CCC(C)O</smiles>

$\underline{N \text {-(2-(4-hydroxypent-1-yn-1-yl)phenyl)-4-methylbenzenesulfonamide(2m) }}$ 
$80 \%$ yield, yellow solid

${ }^{1}$ H NMR (400 MHz, CDCl $) \delta 7.76-7.66(\mathrm{~m}, 2 \mathrm{H}), 7.54(\mathrm{dd}, \mathrm{J}=8.3,0.6 \mathrm{~Hz}, 1 \mathrm{H}), 7.24-$ $7.16(\mathrm{~m}, 4 \mathrm{H}), 6.96(\mathrm{td}, \mathrm{J}=7.6,1.1 \mathrm{~Hz}, 1 \mathrm{H}), 4.09$ (qt, J = 7.3, 3.6 Hz, 1H), 2.63 (dd, J = 16.9, $4.8 \mathrm{~Hz}, 1 \mathrm{H}), 2.53(\mathrm{dd}, \mathrm{J}=16.8,6.6 \mathrm{~Hz}, 1 \mathrm{H}), 2.33(\mathrm{~s}, 3 \mathrm{H}), 1.32(\mathrm{~d}, \mathrm{~J}=6.2 \mathrm{~Hz}, 3 \mathrm{H})$.

${ }^{13}$ C NMR (100 MHz, CDCl3) $\delta$ 143.97, 138.39, 136.34, 131.49, 129.63, 129.08, 127.36, $124.08,119.35,114.55,94.66,77.88,66.38,29.66,23.00,21.59$.

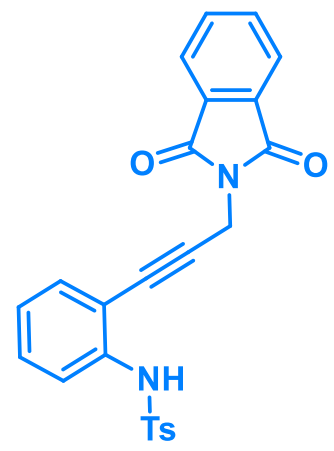

$\underline{N-(2-(3-(1,3-d i o x o i s o i n d o l i n-2-y l) p r o p-1-y n-1-y l) p h e n y l)-4-~}$

methylbenzenesulfonamide(2n)

$63 \%$ yield, white solid

${ }^{1}$ H NMR (400 MHz, CDCl $) \delta 7.93(\mathrm{dd}, \mathrm{J}=5.4,3.1 \mathrm{~Hz}, 2 \mathrm{H}), 7.77(\mathrm{dd}, \mathrm{J}=5.5,3.0 \mathrm{~Hz}, 2 \mathrm{H})$, $7.63(\mathrm{dd}, \mathrm{J}=19.4,8.3 \mathrm{~Hz}, 3 \mathrm{H}), 7.44(\mathrm{~s}, 1 \mathrm{H}), 7.23$ (d, J = 6.4 Hz, 2H), 7.11 (d, J = $8.2 \mathrm{~Hz}$, 2H), $6.97(\mathrm{t}, \mathrm{J}=7.5 \mathrm{~Hz}, 1 \mathrm{H}), 4.63(\mathrm{~s}, 2 \mathrm{H}), 2.29(\mathrm{~s}, 3 \mathrm{H})$.

${ }^{13}$ C NMR (125 MHz, CDCl $) \delta 167.19,143.89,138.64,136.38,134.53,134.38,132.10$, $131.97,130.02$, 129.55, 127.33, 124.31, 123.82, 123.69, 120.30, 113.42, 89.32, 78.77, 28.17, 21.59 .<smiles>CC(C)(C)c1ccc(S(=O)(=O)Nc2ccccc2C#Cc2ccccc2)cc1</smiles>

4-(tert-butyl)-N-(2-(phenylethynyl)phenyl)benzenesulfonamide(2o) $83 \%$ yield, white solid 
${ }^{1} \mathbf{H}$ NMR (400 MHz, CDCl 3 ) $\delta 7.72(\mathrm{~d}, \mathrm{~J}=8.5 \mathrm{~Hz}, 2 \mathrm{H}), 7.64(\mathrm{~d}, \mathrm{~J}=8.2 \mathrm{~Hz}, 1 \mathrm{H}), 7.45$ (dd, J $=6.7,2.9 \mathrm{~Hz}, 2 \mathrm{H}), 7.39(\mathrm{dd}, \mathrm{J}=5.2,3.1 \mathrm{~Hz}, 6 \mathrm{H}), 7.34-7.28(\mathrm{~m}, 1 \mathrm{H}), 7.22(\mathrm{~s}, 1 \mathrm{H}), 7.08(\mathrm{t}, \mathrm{J}$ $=7.5 \mathrm{~Hz}, 1 \mathrm{H}), 1.25(\mathrm{~s}, 9 \mathrm{H})$.

${ }^{13}$ C NMR (100 MHz, CDCl3) $\delta$ 157.05, 137.69, 136.26, 132.12, 131.73, 129.77, 129.16, $128.64,127.14,126.15,124.67,122.13,120.51,114.75,96.15,83.83,35.22,31.08$.<smiles>O=S(=O)(Nc1ccccc1C#Cc1ccccc1)c1ccc(F)cc1F</smiles>

\section{2,4-difluoro- $N$-(2-(phenylethynyl)phenyl)benzenesulfonamide(2q)}

$87 \%$ yield, white solid

${ }^{1}$ H NMR (400 MHz, CDCl3) $\delta 7.91-7.82(\mathrm{~m}, 1 \mathrm{H}), 7.61(\mathrm{~d}, \mathrm{~J}=8.2 \mathrm{~Hz}, 2 \mathrm{H}), 7.57-7.53(\mathrm{~m}$, 2H), $7.45-7.38(\mathrm{~m}, 4 \mathrm{H}), 7.30-7.24(\mathrm{~m}, 1 \mathrm{H}), 7.08(\mathrm{td}, \mathrm{J}=7.6,0.9 \mathrm{~Hz}, 1 \mathrm{H}), 6.94-6.86(\mathrm{~m}$, $1 \mathrm{H}), 6.85-6.76(\mathrm{~m}, 1 \mathrm{H})$.

${ }^{13}$ C NMR (100 MHz, CDCl $) \delta 167.56,167.44,164.99,164.87,161.24,161.11,158.66$, $158.53,136.74,132.77,132.66,132.36,131.74,129.71,129.24,128.66,124.97,122.04$, $119.82,114.59,112.03,111.99,111.81,111.77,106.10,105.84,105.59,96.65,83.25$.

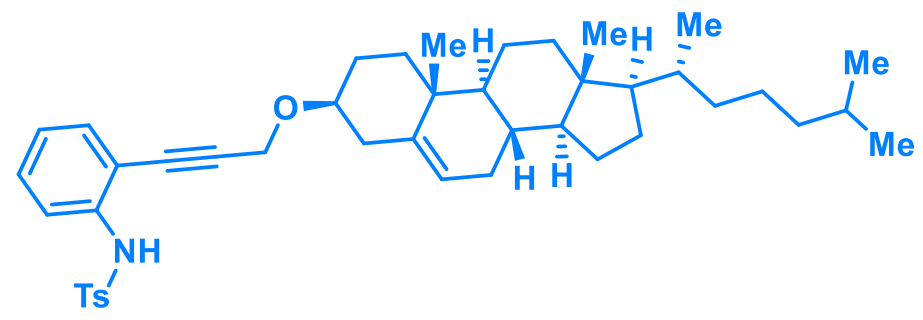

N-(2-(3-(((3S,8S,9S,10R,13R,14S,17R)-10,13-dimethyl-17-((R)-6-methylheptan-2-yl)2,3,4,7,8,9,10,11,12,13,14,15,16,17-tetradecahydro-1H-cyclopenta[a]phenanthren-3yl)oxy)prop-1-yn-1-yl)phenyl)-4-methylbenzenesulfonamide(18a)

$73 \%$ yield, white solid

${ }^{1} \mathbf{H}$ NMR (400 MHz, CDCl3) $\delta 7.68(\mathrm{~d}, \mathrm{~J}=8.3 \mathrm{~Hz}, 2 \mathrm{H}), 7.57(\mathrm{~d}, \mathrm{~J}=8.2 \mathrm{~Hz}, 1 \mathrm{H}), 7.31-7.23$ $(\mathrm{m}, 2 \mathrm{H}), 7.21(\mathrm{~d}, \mathrm{~J}=7.8 \mathrm{~Hz}, 3 \mathrm{H}), 7.00(\mathrm{t}, \mathrm{J}=7.6 \mathrm{~Hz}, 1 \mathrm{H}), 5.41(\mathrm{~d}, \mathrm{~J}=5.1 \mathrm{~Hz}, 1 \mathrm{H}), 4.40$ (s, 2H), $3.48-3.28(\mathrm{~m}, 1 \mathrm{H}), 2.47-2.39(\mathrm{~m}, 1 \mathrm{H}), 2.37(\mathrm{~s}, 3 \mathrm{H}), 2.28(\mathrm{t}, \mathrm{J}=12.2 \mathrm{~Hz}, 1 \mathrm{H}), 2.05-$ $1.81(\mathrm{~m}, 5 \mathrm{H}), 1.60-1.42(\mathrm{~m}, 7 \mathrm{H}), 1.40-1.24(\mathrm{~m}, 4 \mathrm{H}), 1.21-1.05(\mathrm{~m}, 7 \mathrm{H}), 1.03(\mathrm{~s}, 3 \mathrm{H})$, 
$0.97(\mathrm{dd}, \mathrm{J}=16.3,6.4 \mathrm{~Hz}, 3 \mathrm{H}), 0.92(\mathrm{~d}, \mathrm{~J}=6.5 \mathrm{~Hz}, 3 \mathrm{H}), 0.87(\mathrm{~d}, \mathrm{~J}=1.5 \mathrm{~Hz}, 3 \mathrm{H}), 0.86(\mathrm{~d}, \mathrm{~J}=$ $1.5 \mathrm{~Hz}, 3 \mathrm{H}), 0.69$ (s, 3H).

${ }^{13}$ C NMR (100 MHz, CDCl $) \delta 144.13,140.57,138.05,136.25,132.49,129.87,129.75$, $127.49,124.32$, 122.30, 119.58, 113.69, 93.31, 80.37, 78.81, 56.93, 56.31, 55.87, 50.33, $42.48,39.93,39.67,39.00,37.28,37.01,36.34,35.93,32.11,32.05,28.37,28.16,24.44$, 23.97, 22.96, 22.71, 21.73, 21.23, 19.51, 18.87, 12.02 .

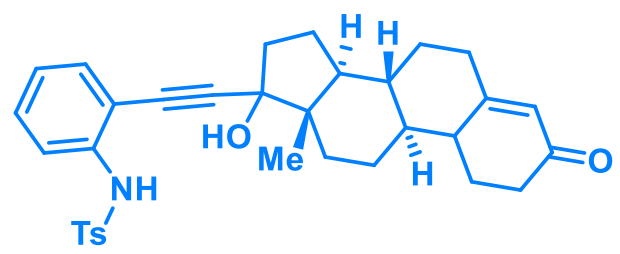

$\underline{N-(2-((8 R, 9 S, 13 S, 14 S)-17-h y d r o x y-13-m e t h y l-3-0 x 0-2,3,6,7,8,9,10,11,12,13,14,15,16,17-}$

tetradecahydro-1H-cyclopenta[a]phenanthren-17-yl)ethynyl)phenyl)-4-

methylbenzenesulfonamide $(18 \mathrm{c})$

$70 \%$ yield, white solid

${ }^{1}$ H NMR (400 MHz, CDCl $) \delta 7.66(\mathrm{~d}, \mathrm{~J}=8.0 \mathrm{~Hz}, 2 \mathrm{H}), 7.54(\mathrm{~d}, \mathrm{~J}=8.2 \mathrm{~Hz}, 1 \mathrm{H}), 7.32$ - 7.21 (m, 3H), 7.19 (d, J = 8.0 Hz, 2H), $7.00(\mathrm{t}, \mathrm{J}=7.6 \mathrm{~Hz}, 1 \mathrm{H}), 5.74(\mathrm{~s}, 1 \mathrm{H}), 2.56$ (s, 1H), 2.48 $2.36(\mathrm{~m}, 3 \mathrm{H}), 2.35(\mathrm{~s}, 3 \mathrm{H}), 2.33-2.25(\mathrm{~m}, 2 \mathrm{H}), 2.16-2.08(\mathrm{~m}, 1 \mathrm{H}), 2.07-2.00(\mathrm{~m}, 1 \mathrm{H})$, $1.90-1.84(\mathrm{~m}, 1 \mathrm{H}), 1.77(\mathrm{~s}, 3 \mathrm{H}), 1.74-1.66(\mathrm{~m}, 2 \mathrm{H}), 1.61(\mathrm{~d}, \mathrm{~J}=8.8 \mathrm{~Hz}, 1 \mathrm{H}), 1.51-1.39$ (m, 3H), $1.20(\mathrm{~s}, 3 \mathrm{H}), 1.13-0.98(\mathrm{~m}, 2 \mathrm{H}), 0.95$ (s, 3H).

${ }^{13}$ C NMR (100 MHz, CDCl3) $\delta$ 199.74, 171.16, 144.20, 137.91, 136.39, 132.15, 129.79, $129.74,127.38,124.35,124.09$, 119.55, 113.93, 100.13, 81.00, 80.36, 53.48, 50.78, 47.32, $39.43,38.76,36.40,35.73,34.05,33.34,32.87,31.58,23.34,21.69,20.92$, 17.59, 12.98 .<smiles>Cc1ccc(C(C)C)c(OCC#Cc2ccccc2NC(F)(F)F)c1</smiles>

$\underline{N-(2-(3-(2-i s o p r o p y l-5-m e t h y l p h e n o x y) p r o p-1-y n-1-y l) p h e n y l)-4-~}$ methylbenzenesulfonamide $(\mathbf{1 8 d})$

$85 \%$ yield, yellow solid

${ }^{1}$ H NMR (400 MHz, CDCl 3$) \delta 7.65-7.62(\mathrm{~m}, 1 \mathrm{H}), 7.59-7.56(\mathrm{~m}, 2 \mathrm{H}), 7.31-7.26(\mathrm{~m}$, 2H), $7.22(\mathrm{dd}, J=8.5,0.6 \mathrm{~Hz}, 1 \mathrm{H}), 7.17(\mathrm{~d}, J=7.7 \mathrm{~Hz}, 1 \mathrm{H}), 7.10(\mathrm{dd}, J=8.5,0.5 \mathrm{~Hz}, 2 \mathrm{H})$, 
$7.00(\mathrm{td}, J=7.6,1.1 \mathrm{~Hz}, 1 \mathrm{H}), 6.85(\mathrm{dd}, J=4.7,3.0 \mathrm{~Hz}, 1 \mathrm{H}), 6.80(\mathrm{~s}, 1 \mathrm{H}), 4.92(\mathrm{~s}, 2 \mathrm{H}), 3.36$ (dt, $J=13.8,6.9 \mathrm{~Hz}, 1 \mathrm{H}), 2.38(\mathrm{~s}, 3 \mathrm{H}), 2.33(\mathrm{~s}, 3 \mathrm{H}), 1.25(\mathrm{~d}, J=6.9 \mathrm{~Hz}, 6 \mathrm{H})$.

${ }^{13}$ C NMR (100 MHz, CDCl $) \delta$ 154.64, 143.95, 139.12, 138.03, 136.56, 135.94, 132.40, $130.01,129.66,129.55,127.47,127.29,126.35,124.26,122.48,119.64,112.85,91.68$, $81.39,56.55,26.52,22.95,21.52,21.43$.<smiles>CNc1ccccc1C#CCOc1ccc(C)cc1</smiles>

\section{4-methyl- $N$-(2-(3-(p-tolyloxy)prop-1-yn-1-yl)phenyl)benzenesulfonamide(26)}

$90 \%$ yield, light yellow solid

${ }^{1}$ H NMR (400 MHz, CDCl3) $\delta 7.56(\mathrm{t}, \mathrm{J}=8.3 \mathrm{~Hz}, 3 \mathrm{H}), 7.26(\mathrm{dd}, \mathrm{J}=7.8,7.0 \mathrm{~Hz}, 2 \mathrm{H}), 7.18$ $(\mathrm{d}, \mathrm{J}=8.3 \mathrm{~Hz}, 2 \mathrm{H}), 7.10(\mathrm{t}, \mathrm{J}=7.0 \mathrm{~Hz}, 3 \mathrm{H}), 7.00(\mathrm{td}, \mathrm{J}=7.7,1.0 \mathrm{~Hz}, 1 \mathrm{H}), 6.96-6.88(\mathrm{~m}$, $2 \mathrm{H}), 4.88(\mathrm{~s}, 2 \mathrm{H}), 2.33$ (s, 3H), $2.33(\mathrm{~s}, 3 \mathrm{H})$.

${ }^{13}$ C NMR (100 MHz, CDCl $) \delta 155.42,144.07,138.18,136.06,132.49,131.29,130.37$, 130.16, 129.68, 127.41, 124.41, 119.96, 114.88, 113.39, 91.35, 81.84, 56.45, 21.66, 20.66.

c) Synthesis of aryl propargyl ether.

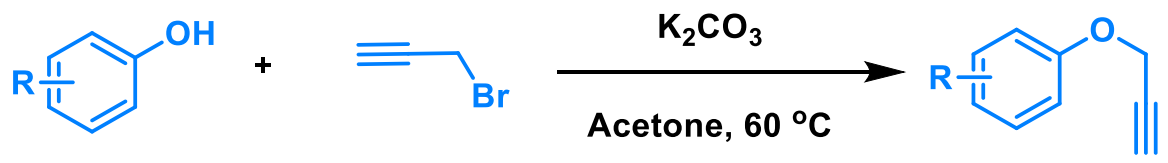

Preparation of (prop-2-yn-1-yloxy) benzene Phenol (10.0 mmol, 1.0 eq.) was suspended in acetone $(20 \mathrm{~mL})$, followed by addition of $\mathrm{K}_{2} \mathrm{CO}_{3}(20.0 \mathrm{mmol}, 2.0$ eq.). The suspension was stirred for $10 \mathrm{~min}$, and then propargyl bromide (15.0 mmol, 1.5 eq.) was added via syringe at room temperature. The reaction mixture was stirred for $5 \mathrm{~h}$ at room temperature. The solvent was removed under reduced pressure and the crude product was purified by flash column chromatography to give the desired product.

Preparation of (3-phenoxyprop-1-yn-1-yl) benzene:<smiles>[R][R]1cccc(OCC#C)c1</smiles>

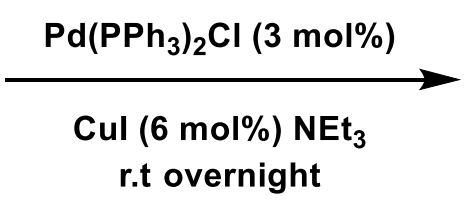<smiles>[R]c1ccc(C#CCCOc2ccccc2)cc1</smiles> 
To a dried schlenk flask was added $\mathrm{Pd}\left(\mathrm{PPh}_{3}\right)_{2} \mathrm{Cl}_{2}$ (3 mol\%), CuI (6 mol\%), iodobenzene (12.0 mmol, 1.2 eq.), (prop-2-yn-1-yloxy) benzene (10.0 mmol, 1.0 eq.) and freshly distilled $\mathrm{Et}_{3} \mathrm{~N}$ with THF under argon. The resulting mixture was stirred for overnight at room temperature. The solvent was removed under reduced pressure and the crude product was purified by flash column chromatography to give the desired product $\mathbf{4}$. The spectroscopic data of unknown starting materials are given below.

Spectroscopic data for unknown compound 4:-<smiles>Cc1ccc(OCC#Cc2ccc([N+](=O)[O-])cc2)cc1</smiles>

\section{1-methyl-4-((3-(4-nitrophenyl)prop-2-yn-1-yl)oxy)benzene(4c)}

$79 \%$ yield, white solid

${ }^{1}$ H NMR (400 MHz, CDCl3) $\delta 8.17(\mathrm{~d}, \mathrm{~J}=8.8 \mathrm{~Hz}, 2 \mathrm{H}), 7.57(\mathrm{~d}, \mathrm{~J}=8.8 \mathrm{~Hz}, 2 \mathrm{H}), 7.13(\mathrm{~d}, \mathrm{~J}=$ $8.3 \mathrm{~Hz}, 2 \mathrm{H}), 6.97-6.88(\mathrm{~m}, 2 \mathrm{H}), 4.91(\mathrm{~s}, 2 \mathrm{H}), 2.31$ (s, 3H).

${ }^{13}$ C NMR (100 MHz, CDCl $) \delta 155.60,147.45,138.78,132.67,131.22,130.14,129.29$, $124.96,123.66,114.93,89.68,85.16,56.66,20.64$.<smiles>Cc1ccc(OCC#Cc2cccc3ccccc23)cc1</smiles>

\section{1-(3-(p-tolyloxy)prop-1-yn-1-yl)naphthalene(4d)}

$83 \%$ yield, brown sticky solid.

${ }^{1}$ H NMR (400 MHz, CDCl3) $\delta 8.26(\mathrm{~d}, \mathrm{~J}=7.6 \mathrm{~Hz}, 1 \mathrm{H}), 7.85(\mathrm{dd}, \mathrm{J}=8.9,4.1 \mathrm{~Hz}, 2 \mathrm{H}), 7.71$ $(\mathrm{d}, \mathrm{J}=7.1 \mathrm{~Hz}, 1 \mathrm{H}), 7.59-7.48(\mathrm{~m}, 2 \mathrm{H}), 7.47-7.38(\mathrm{~m}, 1 \mathrm{H}), 7.18(\mathrm{~d}, \mathrm{~J}=8.4 \mathrm{~Hz}, 2 \mathrm{H}), 7.06$ $(\mathrm{d}, \mathrm{J}=8.4 \mathrm{~Hz}, 2 \mathrm{H}), 5.07$ (s, 2H), $2.36(\mathrm{~s}, 3 \mathrm{H})$. 
${ }^{13}$ C NMR (100 MHz, CDCl3) $\delta 155.81,133.44,133.18,130.92,130.84,130.07,129.20$, $128.34,126.92,126.53,126.23,125.22,120.12,115.24,89.17,85.33,57.11,20.66$.<smiles>CC(C)(c1ccccc1)c1ccc(OCC#CPc2ccccc2)cc1</smiles>

\section{1-((3-phenylprop-2-yn-1-yl)oxy)-4-(2-phenylpropan-2-yl)benzene (4f)}

$83 \%$ yield, white solid

${ }^{1}$ H NMR (400 MHz, CDCl3) $\delta 7.49-7.43(\mathrm{~m}, 2 \mathrm{H}), 7.35-7.23(\mathrm{~m}, 7 \mathrm{H}), 7.21-7.15(\mathrm{~m}$, $3 \mathrm{H}), 6.95$ (dd, J = 9.4, 2.6 Hz, 2H), 4.90 (s, 2H), 1.69 (s, 6H).

13C NMR (100 MHz, CDCl $) \delta 155.92,150.96,143.84,131.98,128.76,128.41,128.11$, $127.96,126.89,125.71,122.51,114.54,87.16,84.31,56.90,42.51,31.02$.

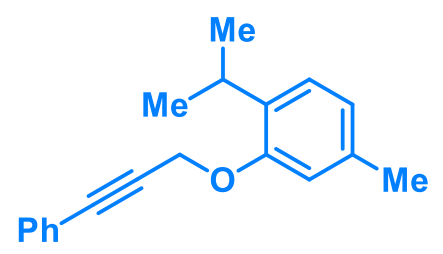

\section{1-isopropyl-4-methyl-2-((3-phenylprop-2-yn-1-yl)oxy)benzene(18e)}

$73 \%$ yield, light brown solid

${ }^{1}$ H NMR (400 MHz, CDCl 3$) \delta 7.59-7.50(\mathrm{~m}, 2 \mathrm{H}), 7.39$ (d, $\left.J=4.2 \mathrm{~Hz}, 3 \mathrm{H}\right), 7.24(\mathrm{~d}, J=7.6$ $\mathrm{Hz}, 1 \mathrm{H}), 6.97(\mathrm{~s}, 1 \mathrm{H}), 6.91(\mathrm{~d}, J=7.4 \mathrm{~Hz}, 1 \mathrm{H}), 5.01(\mathrm{~s}, 2 \mathrm{H}), 3.58-3.38(\mathrm{~m}, 1 \mathrm{H}), 2.45$ (s, $3 \mathrm{H}), 1.34(\mathrm{~d}, J=6.9 \mathrm{~Hz}, 6 \mathrm{H})$.

${ }^{13}$ C NMR (100 MHz, CDCl $) \delta 155.14,136.34,134.89,131.90,128.65,128.37,126.15$, $122.63,122.23,113.52,86.84,84.75,57.18,26.55,23.06,21.48$.<smiles>C(#Cc1ccc2c(c1)OCO2)COc1ccccc1</smiles>

\section{5-((3-phenylprop-2-yn-1-yl)oxy)benzo[d][1,3] dioxole(18f)}

93\% yield, light yellow sticky solid.

${ }^{1}$ H NMR (400 MHz, CDCl3) $\delta 7.47-7.42(\mathrm{~m}, 2 \mathrm{H}), 7.35-7.27(\mathrm{~m}, 3 \mathrm{H}), 6.74(\mathrm{~d}, \mathrm{~J}=8.5 \mathrm{~Hz}$, $1 \mathrm{H}), 6.63(\mathrm{~d}, \mathrm{~J}=2.5 \mathrm{~Hz}, 1 \mathrm{H}), 6.48(\mathrm{dd}, \mathrm{J}=8.5,2.5 \mathrm{~Hz}, 1 \mathrm{H}), 5.93(\mathrm{~s}, 2 \mathrm{H}), 4.84(\mathrm{~s}, 2 \mathrm{H})$.

${ }^{13}$ C NMR (100 MHz, CDCl $) \delta 153.42,148.38,142.41,131.94,128.80,128.42,122.42$, $108.05,106.75,101.36,98.91,87.29,84.12,57.91$. 


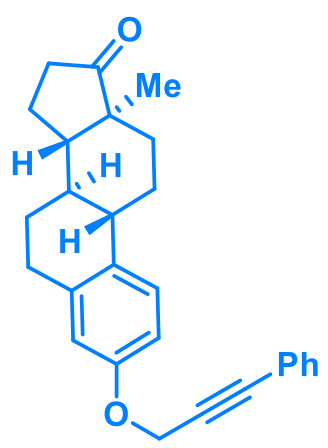

(8R,9S,13S,14S)-13-methyl-3-((3-phenylprop-2-yn-1-yl)oxy)-6,7,8,9,11,12,13,14,15,16decahydro-17H-cyclopenta[a]phenanthren-17-one(18g)

$69 \%$ yield, white sticky solid.

${ }^{1}$ H NMR (400 MHz, CDCl 3$) \delta 7.47-7.41$ (m, 2H), 7.40 - 7.35 (m, 1H), 7.33 - 7.30 (m, 2H), $7.23(\mathrm{~d}, \mathrm{~J}=8.6 \mathrm{~Hz}, 1 \mathrm{H}), 6.85(\mathrm{dd}, \mathrm{J}=8.6,2.8 \mathrm{~Hz}, 1 \mathrm{H}), 6.78(\mathrm{~d}, \mathrm{~J}=2.7 \mathrm{~Hz}, 1 \mathrm{H}), 4.88(\mathrm{~s}$, 2H), $2.92(\mathrm{dd}, \mathrm{J}=9.6,4.8 \mathrm{~Hz}, 2 \mathrm{H}), 2.51(\mathrm{dd}, \mathrm{J}=18.8,8.5 \mathrm{~Hz}, 1 \mathrm{H}), 2.44-2.37(\mathrm{~m}, 1 \mathrm{H}), 2.31$ $-2.23(\mathrm{~m}, 1 \mathrm{H}), 2.18-2.09(\mathrm{~m}, 1 \mathrm{H}), 2.05-1.94(\mathrm{~m}, 2 \mathrm{H}), 1.65-1.58(\mathrm{~m}, 3 \mathrm{H}), 1.55-1.41$ (m, 4H), $0.91(\mathrm{~s}, 3 \mathrm{H})$.

${ }^{13}$ C NMR (100 MHz, CDCl $) \delta 221.14,155.98,137.97,132.96,131.96,128.77,128.40$, $126.50,122.49,115.19,112.58,87.14,84.28,56.78,50.54,48.15,44.13,38.43,36.01,31.70$, 29.81, 26.66, 26.02, 21.72, 13.99 .

d) Typical procedure for the synthesis of compound 6 .

i) Synthesis of 2-(Prop-2'-ynyloxy)nitrobenzene.

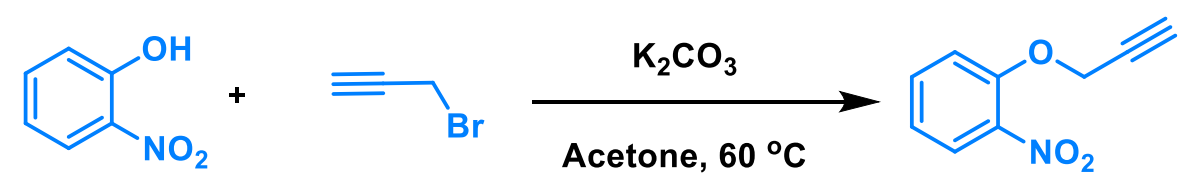

A mixture of 2-nitrophenol $(5.0 \mathrm{~g}, 35.9 \mathrm{mmol})$ and anhydrous $\mathrm{K}_{2} \mathrm{CO}_{3}(7.45 \mathrm{~g}, 35.9 \mathrm{mmol})$ in dry acetone $(30 \mathrm{~mL})$ was stirred for $2 \mathrm{~h}$ at room temperature. Propargyl bromide $(5.12 \mathrm{~g}$, $43.08 \mathrm{mmol})$ in dry acetone $(10 \mathrm{~mL})$ was added during $20 \mathrm{~min}$. The whole mixture was heated under reflux for $16 \mathrm{~h}$ with constant stirring under nitrogen atmosphere. Acetone was removed from the mixture, and the residue was poured in distilled water $(100 \mathrm{~mL})$ and extracted with chloroform $(3 \times 50 \mathrm{~mL})$. The combined organic layer was washed with water $(100 \mathrm{~mL})$ and dried over anhydrous $\mathrm{Na}_{2} \mathrm{SO}_{4}$. After removal of solvent, the residue was purified through column chromatography over silica gel using chloroform-petroleum ether $(1: 1)$ as eluent. Finally, the product was crystallized from petroleum etherchloroform. A colorless white crystalline solid. 
ii) Synthesis of 2-(Prop-2'-ynyloxy)-aniline. It was prepared by the reduction of compound 2-(Prop-2' -ynyloxy) nitrobenzene with $\mathrm{Fe} / \mathrm{AcOH}$ following the usual procedure. ${ }^{2}$

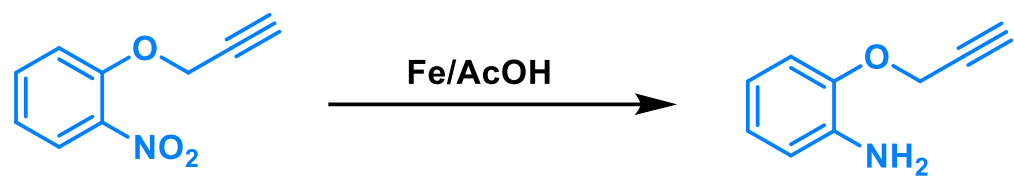

iii) Palladium catalyzed coupling of substituted iodobenzene with aniline and protection with tosyl chloride.<smiles>C#CCOc1ccccc1N</smiles><smiles>[R]Cc1ccc(I)cc1</smiles>
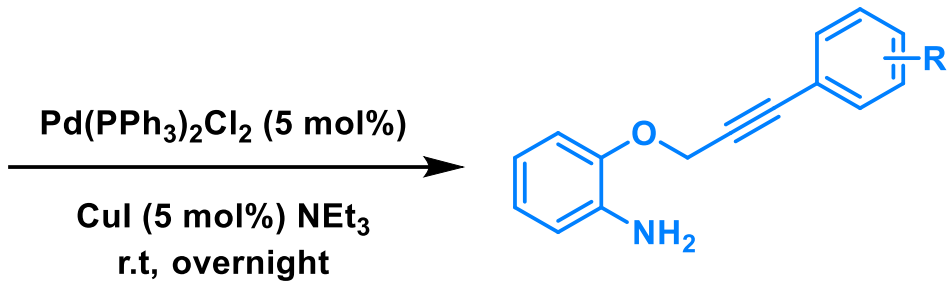

\section{Step-1 ${ }^{\text {st }}$}

A mixture of substituted iodobenzene $(2.03 \mathrm{mmol}),\left(\mathrm{PPh}_{3}\right)_{2} \mathrm{PdCl}_{2}(5 \mathrm{~mol} \%)$, and $\mathrm{CuI}(5$ mol\%) in triethylamine $(9 \mathrm{~mL})$ was stirred under $\mathrm{N}_{2}$ atmosphere for $20 \mathrm{~min}$. Then 2-(prop-2'ynyloxy) aniline (360 mg, $2.44 \mathrm{mmol})$ in triethylamine $(3 \mathrm{~mL})$ was added very slowly. The resulting reaction mixture was stirred at room temperature for $16 \mathrm{~h}$ under $\mathrm{N}_{2}$ atmosphere. After the removal of triethylamine, the reaction mixture was poured in water $(50 \mathrm{~mL})$ and extracted with chloroform $(3 \times 40 \mathrm{~mL})$. The combined chloroform layer was washed with water and dried over anhydrous $\mathrm{Na}_{2} \mathrm{SO}_{4}$. After the removal of solvent, the residue was chromatographed over silica gel using chloroform/petroleum ether (75/25, V/V) as eluent, affording desired product which directly subjected for $2^{\text {nd }}$ step.
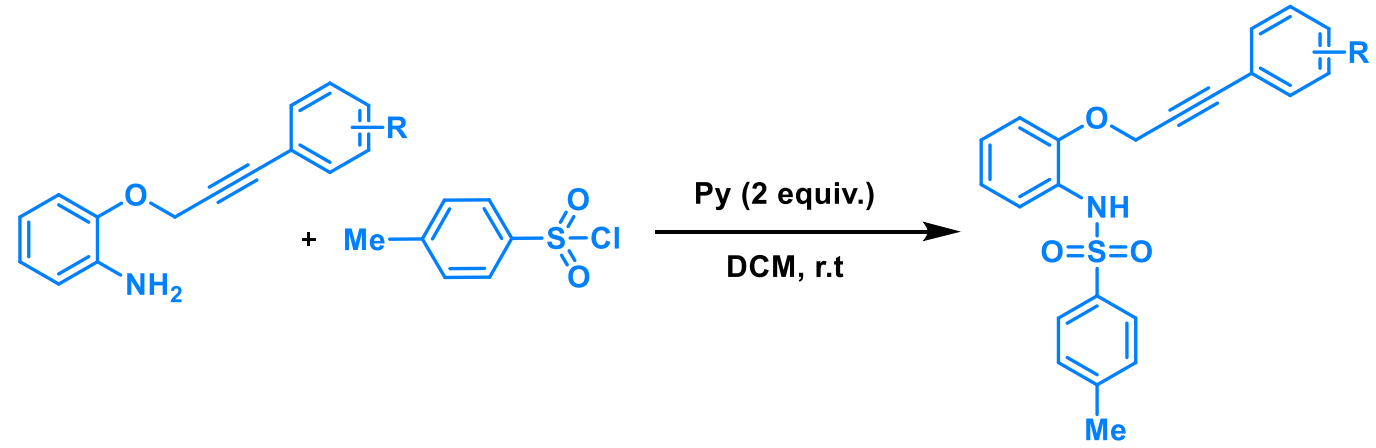

Step $2^{\text {nd }}$ - To a solution of 2-(ethynyl)benzenamine (1 equiv.) and pyridine (2 equiv.) in $20 \mathrm{~mL}$ of dichloromethane was added 4-methylbenzene-1-sulfonyl chloride or substituted benzene1-sulfonyl chloride ( 1 equiv.) at $0{ }^{\circ} \mathrm{C}$, then the reaction solution was warmed to room temperature. After the reaction was complete (about $10 \mathrm{~h}$ ) as monitored by TLC, water was added, and the mixture was extracted with ether. The combined organic phase was washed with brine, dried over anhydrous $\mathrm{Na}_{2} \mathrm{SO}_{4}$. The solvent was evaporated under the reduced 
pressure and the residue was purified by chromatography on silica gel to afford the compound 6. The spectroscopic data of unknown starting materials are given below.

Spectroscopic data for unknown compounds 6:-

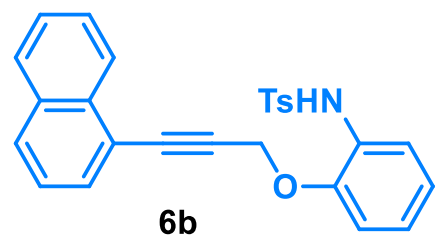

4-methyl- $N$-(2-((3-(naphthalen-1-yl)prop-2-yn-1-yl)oxy)phenyl)benzenesulfonamide(6b) $78 \%$ yield, white solid

${ }^{1} \mathbf{H}$ NMR (400 MHz, CDCl $) \delta 7.95(\mathrm{~d}, J=8.2 \mathrm{~Hz}, 1 \mathrm{H}), 7.85(\mathrm{~d}, J=8.4 \mathrm{~Hz}, 2 \mathrm{H}), 7.70-7.59$ (m, 4H), $7.53-7.40(\mathrm{~m}, 3 \mathrm{H}), 7.18(\mathrm{~s}, 1 \mathrm{H}), 7.14-6.95(\mathrm{~m}, 5 \mathrm{H}), 4.90(\mathrm{~s}, 2 \mathrm{H}), 2.03(\mathrm{~s}, 3 \mathrm{H})$.

${ }^{13}$ C NMR (100 MHz, CDCl $) \delta 147.80,143.71,136.11,133.27,133.14,130.76,129.48$, $129.44,128.37,127.28,127.04,126.78,126.62,125.95,125.41,125.19,122.12,121.83$, $119.59,112.79,87.72,86.18,57.40,21.28$<smiles>Nc1ccccc1OCC#Cc1cccc(C(F)(F)F)c1</smiles>

4-methyl- $N$-(2-((3-(3-(trifluoromethyl)phenyl)prop-2-yn-1yl)oxy)phenyl)benzenesulfonamide $(6 \mathrm{c})$

$70 \%$ yield, white solid.

${ }^{1}$ H NMR (400 MHz, CDCl 3$) \delta 7.67(\mathrm{~d}, J=7.2 \mathrm{~Hz}, 3 \mathrm{H}), 7.57$ (d, $\left.J=6.4 \mathrm{~Hz}, 3 \mathrm{H}\right), 7.46$ (s, 1H), $7.23-7.10(\mathrm{~m}, 3 \mathrm{H}), 7.06(\mathrm{~s}, 1 \mathrm{H}), 6.94(\mathrm{~d}, J=10.8 \mathrm{~Hz}, 2 \mathrm{H}), 4.73(\mathrm{~s}, 2 \mathrm{H}), 2.27(\mathrm{~s}, 3 \mathrm{H})$. ${ }^{13}$ C NMR (100 MHz, CDCl $) \delta 147.77,143.82,136.35,135.00,131.29,129.50,129.10$, $128.60(\mathrm{q}, J=4.1 \mathrm{~Hz}), 127.39,126.63,125.60(\mathrm{q}, J=3.4 \mathrm{~Hz}), 125.36,125.03,122.95$, $122.32,122.23,121.58,112.40,86.31,84.64,57.20,21.50$. 
3) Optimization of the reaction conditions ${ }^{a}$

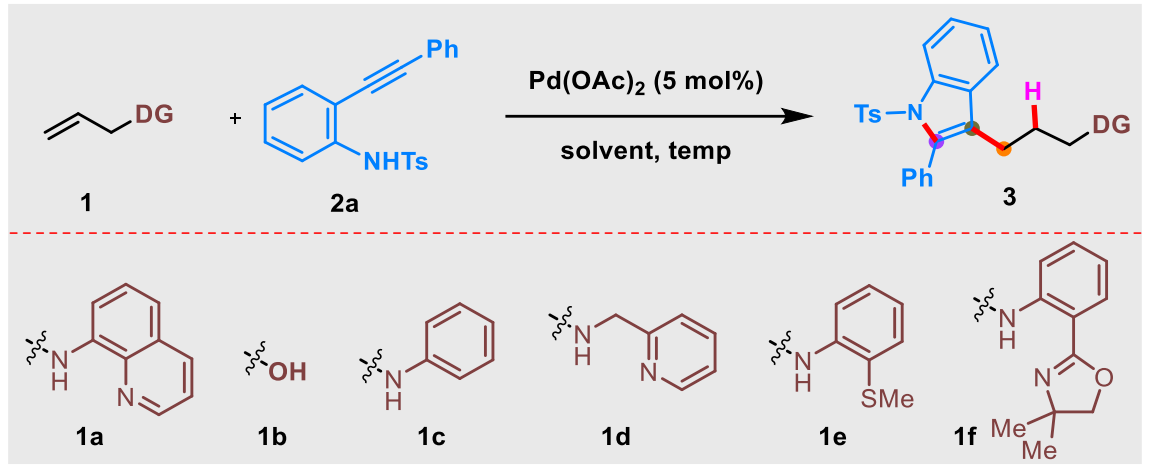

\begin{tabular}{|c|c|c|c|c|}
\hline S. No & DG & Temp & Solvent & Yield $(\%)^{\mathrm{t}}$ \\
\hline 1 & $1 \mathrm{a}$ & 80 & $\mathrm{CH}_{3} \mathrm{CN}$ & Traces \\
\hline 2 & $1 \mathrm{a}$ & 80 & DCE & n.r \\
\hline 3 & $1 \mathrm{a}$ & 80 & 1,4 dioxane & Traces \\
\hline 4 & $1 \mathbf{a}$ & 80 & $t$-Amyl-OH & 20 \\
\hline 5 & $1 \mathbf{a}$ & 80 & $t$-Amyl-OH & 38 \\
\hline $6^{\mathrm{c}}$ & $1 \mathbf{a}$ & 80 & TFE & 47 \\
\hline $7^{\mathrm{c}}$ & $1 \mathbf{a}$ & 80 & HFIP & 60 \\
\hline 8 & $1 \mathbf{a}$ & 80 & $\mathrm{AcOH}$ & 20 \\
\hline 9 & $\mathbf{1 a}$ & 80 & HFIP & 75 \\
\hline 10 & $1 \mathbf{a}$ & r.t. & $t$-Amyl-OH & traces \\
\hline 11 & $1 \mathbf{a}$ & 50 & HFIP & 64 \\
\hline 12 & $1 \mathbf{a}$ & 100 & HFIP & 72 \\
\hline 13 & $1 b$ & 80 & HFIP & n.r \\
\hline 14 & $1 c$ & 80 & HFIP & n.r \\
\hline 15 & 1d & 80 & HFIP & 10 \\
\hline 16 & $1 e$ & 80 & HFIP & 5 \\
\hline 17 & 1f & 80 & HFIP & 10 \\
\hline $18^{\mathrm{d}}$ & $1 \mathbf{a}$ & 80 & HFIP & 60 \\
\hline $19^{\mathrm{e}}$ & $1 \mathbf{a}$ & 80 & HFIP & 55 \\
\hline $20^{f}$ & $1 \mathbf{a}$ & 80 & HFIP & $98(93)^{\mathrm{g}}$ \\
\hline $21^{\mathrm{f}, \mathrm{h}}$ & $1 \mathbf{a}$ & 80 & HFIP & 97 \\
\hline $22^{\mathrm{f}, \mathrm{i}}$ & $1 \mathbf{a}$ & 80 & HFIP & 95 \\
\hline $23^{\mathrm{j}}$ & $1 \mathbf{a}$ & 80 & HFIP & n.r \\
\hline
\end{tabular}

Reaction conditions: [a] $0.1 \mathrm{mmol}$ of $\mathbf{1}, 0.12 \mathrm{mmol}$ of $\mathbf{2 a}, 5 \mathrm{~mol} \%$ $\mathrm{Pd}(\mathrm{OAc})_{2}$ in $1 \mathrm{~mL}$ solvent. [b] NMR yield using $0.1 \mathrm{mmol}$ of $1,3,5-$ 
trimethoxybenzene as an internal standard. [c] $30 \mathrm{~mol} \%$ of $\mathrm{AcOH}$ [d] $5 \mathrm{~mol}_{\%} \mathrm{PdCl}_{2}$ [e] $5 \mathrm{~mol} \% \mathrm{Pd}\left(\mathrm{PhCN}_{2} \mathrm{Cl}_{2}\right.$. [f] $0.2 \mathrm{mmol}$ of $\mathbf{2 a}$ [g] Yield in parenthesis refers to isolated yield. [h] reaction under $\mathrm{N}_{2}$. [i] reaction under $\mathrm{O}_{2}$. [j] no catalyst

\section{4) General procedure for hydro-heteroarylation of unactivated alkenes.}

a) General procedure for "palladium catalyzed hydroindolation of alkenamides.

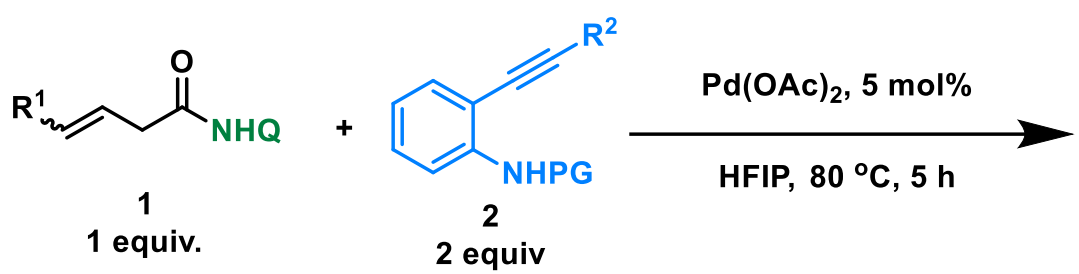<smiles>[R]c1c(C([R1])CCC(=O)NO)c2ccccc2n1[Po]</smiles>

To an oven dried screw-cap reaction tube equipped with stir bar, alkenamide $1(0.1 \mathrm{mmol}), \mathbf{2}$ $(0.2 \mathrm{mmol})$ and $\mathrm{Pd}(\mathrm{OAc})_{2}$ catalyst $(5 \mathrm{~mol} \%)$ were added sequentially, followed by HFIP (1 $\mathrm{mL}$ ). The reaction mixture was heated for $5 \mathrm{~h}$ at $80{ }^{\circ} \mathrm{C}$. The completion of the reaction was confirmed by checking TLC under UV detector. Then, the organic phase was evaporated under reduced pressure and the product was purified by using silica-gel column chromatography (eluent:Hexane/Ethyl acetate $=8 / 2$ )

b) General procedure for "palladium catalyzed hydrochromenation or hydro-heteroarylation of alkenamides via C-cyclization.
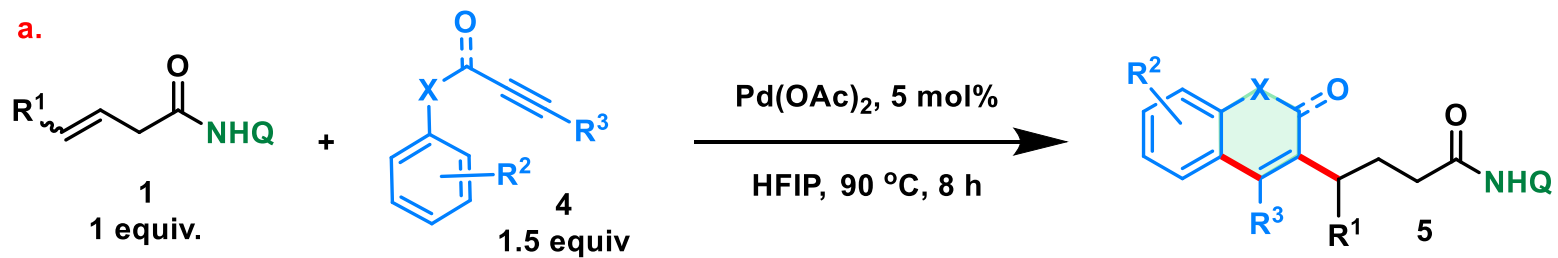

To an oven dried screw-cap reaction tube equipped with stir bar, alkenamide 1 (0.1 mmol), 2 $(0.15 \mathrm{mmol})$ and $\mathrm{Pd}(\mathrm{OAc})_{2}$ catalyst $(5 \mathrm{~mol} \%)$ were added sequentially, followed by HFIP (1 $\mathrm{mL}$ ). The reaction mixture was heated for $8 \mathrm{~h}$ at $90{ }^{0} \mathrm{C}$. The completion of the reaction was confirmed by checking TLC under UV detector. Then, the organic phase was evaporated under reduced pressure and the product was purified by using silica-gel column chromatography (eluent:Hexane/Ethyl acetate $=8: 1$ ) 


\section{Gram scale synthesis and removal of direction group.}

a. Gram scale: A mixture of alkenamide 1u (1g, $3.47 \mathrm{mmol})$, 2a (2.4 g $6.93 \mathrm{mmol})$, $\mathrm{Pd}(\mathrm{OAc})_{2}$ catalyst $(5 \mathrm{~mol} \%)$ were added sequentially, followed by HFIP ( $\left.15 \mathrm{~mL}\right)$. The reaction mixture was heated for $5 \mathrm{~h}$ at $80{ }^{\circ} \mathrm{C}$. The completion of the reaction was confirmed by checking TLC under UV detector. Afterwards, the solvent was evaporated under reduced pressure, and the residue was adsorbed onto small amounts of silica. The purification was performed by flash column chromatography on silica gel (eluent: EtOAc/petroleum ether = 2:8) affording $3 \mathbf{u}(1.89 \mathrm{~g}, 85 \%)$.

\section{b. Removal of directing group.}

In an oven dried reaction tube $0.1 \mathrm{mmol}$ of $\mathbf{3}(\mathbf{3 m}$ or $\mathbf{3 u})$ was taken. Then 3 equiv. $p$ toluenesulphonic acid and $1 \mathrm{~mL}$ methanol was added to it. The mixture was stirred in a preheated oil bath at $90{ }^{\circ} \mathrm{C}$ for 24 hours. After completion reaction was cooled to room temperature, filtered through celite and evaporated under vacuum. Pure product of 21 (21a or 21b) was isolated by column chromatography through a silica gel column (mesh 100-200). Eluent: ethyl acetate / petroleum ether $(5: 95 \mathrm{v} / \mathrm{v})$

\section{Control experiment.}

a. Comparative reactivity between activated alkene and directed unactivated alkene for hydroheterocarbofunctionalization.

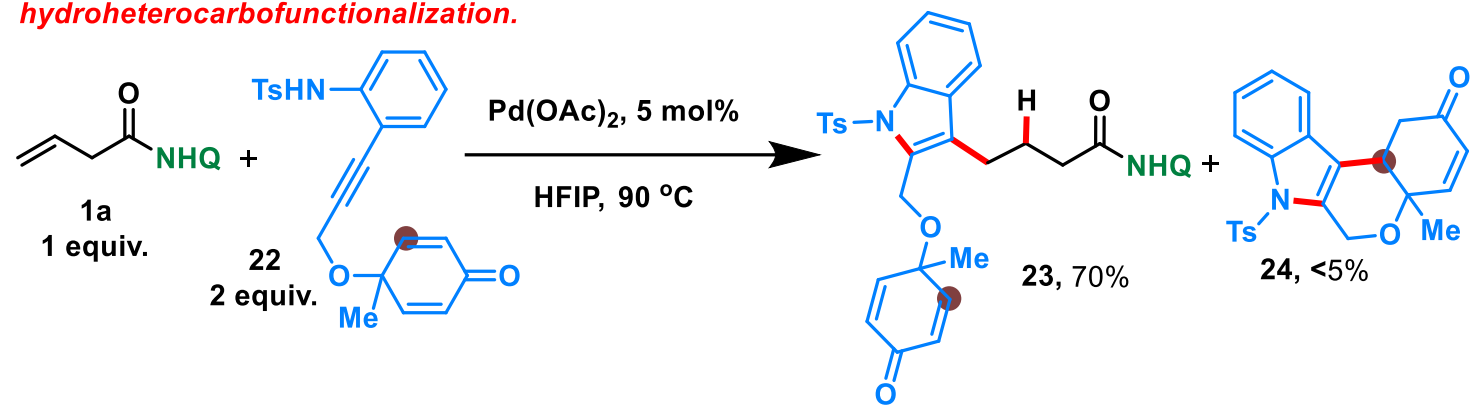

A mixture of indole 1a $(0.1 \mathrm{mmol})$, activated alkene tethered $22(0.2 \mathrm{mmol}), \mathrm{Pd}(\mathrm{OAc})_{2}$ catalyst $(5 \mathrm{~mol} \%)$ were added sequentially, followed by HFIP $(1 \mathrm{~mL})$ and the mixture was stirred at $80{ }^{\circ} \mathrm{C}$ for $6 \mathrm{~h}$ under air atmosphere. Afterwards, the solvent was evaporated under reduced pressure, and the residue was adsorbed onto small amounts of silica. The purification was performed by flash column chromatography on silica gel (eluent: EtOAc/petroleum ether $=2: 8$ ) affording $\mathbf{2 3}$ over $\mathbf{2 4}$. The formation of $\mathbf{2 3}$ is clearly suggesting that, directed intermolecular hydroindolation of unactivated alkene is most preferred as compare to intramolecular hydroindolation of activated $\alpha, \beta$-unsaturated carbonyl. 
b. Palladium catalyzed direct hydroindolation of unactivated alkene with $\mathbf{N}$-tosyl-2-phenyl indole
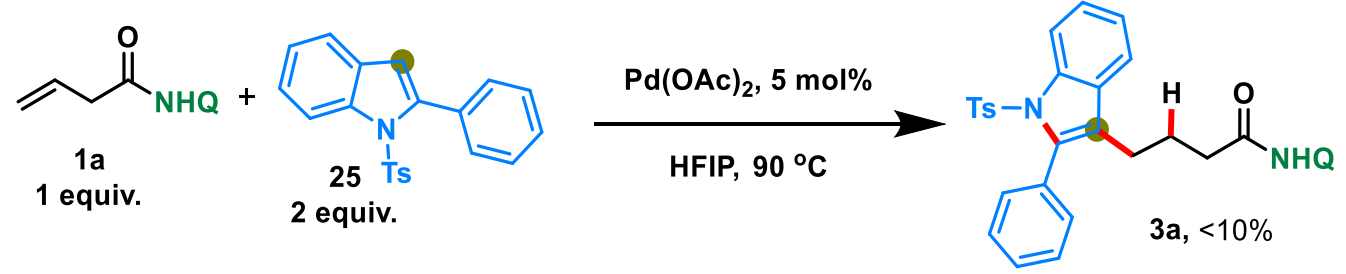

A mixture of $1 \mathbf{a}(0.1 \mathrm{mmol})$, indole $25(0.2 \mathrm{mmol}), \mathrm{Pd}(\mathrm{OAc})_{2}$ catalyst $(5 \mathrm{~mol} \%)$ were added sequentially, followed by HFIP ( $1 \mathrm{~mL})$ and the mixture was stirred at $80{ }^{\circ} \mathrm{C}$ for $6 \mathrm{~h}$ under air atmosphere. The target product was not been observed, suggesting that, palladium-indole intermediate undergoing migratory insertion rather than nucleophilic attack of indole on alkene.

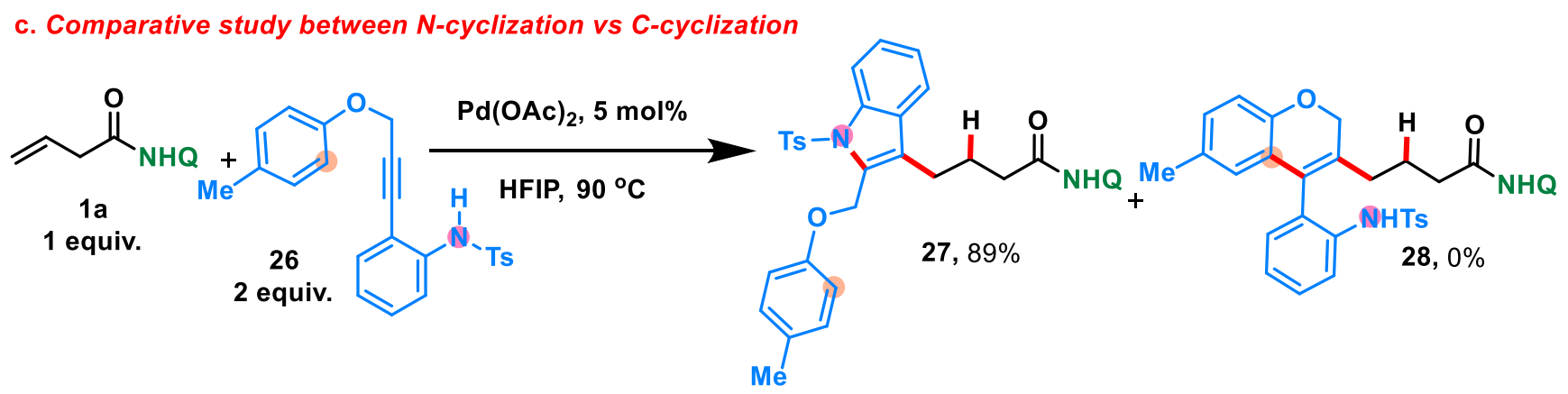

Competitive experiment for carbopalladation and aminopalladation was carried out by using 4-methyl- $N$-(2-(3-((1-methyl-4-oxocyclohexa-2,5-dien-1-yl)oxy)prop-1-yn-1-yl)phenyl)

benzenesulfonamide $\mathbf{2 6}$ and 1a as model substrate in standard condition and found the exclusive formation of cascade aminopalladation product 27 (89\%).

\section{Spectroscopic data for new compounds}<smiles>O=C(CCc1c(-c2ccccc2)n([125I])c2ccccc12)NO</smiles>

\section{4-(2-phenyl-1-tosyl-1H-indol-3-yl)- $N$-(quinolin-8-yl)butanamide (3a)}

$52.1 \mathrm{mg}, 93 \%$ yield, white solid.

$\boldsymbol{R}_{\boldsymbol{f}}: 0.40$ (hexane/ethyl acetate, $6: 4 \mathrm{v} / \mathrm{v}$ ).

${ }^{1}$ H NMR (400 MHz, CDCl3) $\delta 9.65(\mathrm{~s}, 1 \mathrm{H}), 8.77(\mathrm{dd}, J=4.2,1.5 \mathrm{~Hz}, 1 \mathrm{H}), 8.72(\mathrm{dd}, J=7.1$, $1.2 \mathrm{~Hz}, 1 \mathrm{H}), 8.35(\mathrm{~d}, J=8.3 \mathrm{~Hz}, 1 \mathrm{H}), 8.13(\mathrm{dd}, J=8.3,1.5 \mathrm{~Hz}, 1 \mathrm{H}), 7.60(\mathrm{~d}, J=7.6 \mathrm{~Hz}, 1 \mathrm{H})$, $7.54-7.46(\mathrm{~m}, 2 \mathrm{H}), 7.43(\mathrm{dd}, J=8.3,4.2 \mathrm{~Hz}, 1 \mathrm{H}), 7.41-7.37(\mathrm{~m}, 1 \mathrm{H}), 7.36(\mathrm{~s}, 5 \mathrm{H}), 7.33-$ 
$7.25(\mathrm{~m}, 3 \mathrm{H}), 7.05(\mathrm{~d}, J=8.1 \mathrm{~Hz}, 2 \mathrm{H}), 2.70-2.59(\mathrm{~m}, 2 \mathrm{H}), 2.41(\mathrm{t}, J=7.3 \mathrm{~Hz}, 2 \mathrm{H}), 2.26(\mathrm{~s}$, $3 \mathrm{H}), 2.03-1.93(\mathrm{~m}, 2 \mathrm{H})$.

${ }^{13}$ C NMR (100 MHz, CDCl $) \delta 171.02,148.15,144.51,138.31,137.41,137.07,136.41$, $135.26,134.45,131.40,131.30,130.85$, 129.28, 128.54, 127.97, 127.56, 127.40, 126.81, 124.97, 124.01, 123.42, 121.65, 121.47, 119.50, 116.45, 116.23, 37.27, 25.44, 23.81, 21.52.

HRMS (ESI-TOF) m/z: [M+H] $]^{+}$calcd for $\mathrm{C}_{34} \mathrm{H}_{30} \mathrm{~N}_{3} \mathrm{O}_{3} \mathrm{~S} 560.2002$; Found 560.2012.

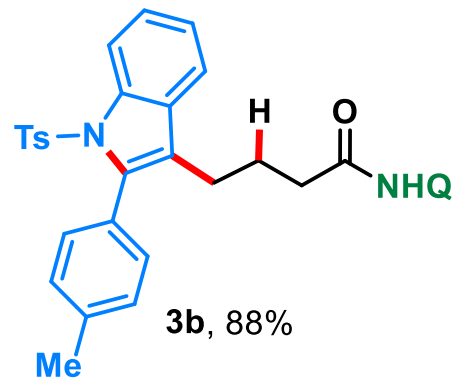

\section{$\underline{N \text {-(quinolin-8-yl)-4-(2-(p-tolyl)-1-tosyl-1H-indol-3-yl)butanamide (3b) }}$}

$50.5 \mathrm{mg}, 88 \%$ yield, white solid.

$\boldsymbol{R}_{\boldsymbol{f}}: 0.40$ (hexane/ethyl acetate, $6: 4 \mathrm{v} / \mathrm{v}$ ).

${ }^{1}$ H NMR (400 MHz, CDCl $) \delta 9.62(\mathrm{~s}, 1 \mathrm{H}), 8.79(\mathrm{dd}, J=4.1,1.4 \mathrm{~Hz}, 1 \mathrm{H}), 8.71(\mathrm{~d}, J=6.9$ $\mathrm{Hz}, 1 \mathrm{H}), 8.33(\mathrm{~d}, J=8.3 \mathrm{~Hz}, 1 \mathrm{H}), 8.16(\mathrm{~d}, J=8.2 \mathrm{~Hz}, 1 \mathrm{H}), 7.61-7.42(\mathrm{~m}, 4 \mathrm{H}), 7.40-7.21$ $(\mathrm{m}, 6 \mathrm{H}), 7.14(\mathrm{~d}, J=7.8 \mathrm{~Hz}, 2 \mathrm{H}), 7.06(\mathrm{~d}, J=8.1 \mathrm{~Hz}, 2 \mathrm{H}), 2.63(\mathrm{t}, J=7.6 \mathrm{~Hz}, 2 \mathrm{H}), 2.40(\mathrm{t}, J$ $=7.3 \mathrm{~Hz}, 2 \mathrm{H}), 2.31(\mathrm{~s}, 3 \mathrm{H}), 2.27(\mathrm{~s}, 3 \mathrm{H}), 2.04-1.93(\mathrm{~m}, 2 \mathrm{H})$.

${ }^{13}$ C NMR (100 MHz, CDCl $) \delta 171.13,148.20,144.50,138.44,138.34,137.35,136.48$, 135.21 , 134.48, 131.16, 130.98, 129.29, 128.40, 128.02, 127.48, 126.87, 124.89, 124.02, $123.23,121.71,121.50,119.43,116.49,116.30,37.19,25.37,23.80,21.60,21.46$.

HRMS (ESI-TOF) m/z: $[\mathrm{M}+\mathrm{H}]^{+}$calcd for $\mathrm{C}_{35} \mathrm{H}_{32} \mathrm{~N}_{3} \mathrm{O}_{3} \mathrm{~S}$ 574.2159; Found 574.2158.

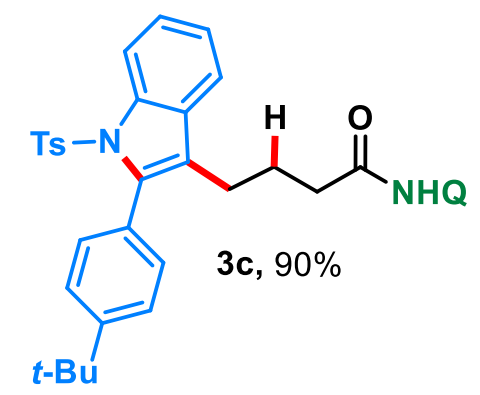

4-(2-(4-(tert-butyl)phenyl)-1-tosyl-1H-indol-3-yl)- $N$-(quinolin-8-yl)butanamide (3c)

$55.4 \mathrm{mg}, 90 \%$ yield, white solid. 
$\boldsymbol{R}_{f}: 0.40$ (hexane/ethyl acetate, 6:4 v/v).

${ }^{1}$ H NMR (400 MHz, CDCl 3$) \delta 9.67(\mathrm{~s}, 1 \mathrm{H}), 8.79$ (dd, $\left.J=4.1,1.4 \mathrm{~Hz}, 1 \mathrm{H}\right), 8.73$ (dd, $J=6.9$, $1.5 \mathrm{~Hz}, 1 \mathrm{H}), 8.33(\mathrm{~d}, J=8.3 \mathrm{~Hz}, 1 \mathrm{H}), 8.16(\mathrm{dd}, J=8.3,1.3 \mathrm{~Hz}, 1 \mathrm{H}), 7.57(\mathrm{~d}, J=7.6 \mathrm{~Hz}, 1 \mathrm{H})$, $7.55-7.43(\mathrm{~m}, 3 \mathrm{H}), 7.37-7.24(\mathrm{~m}, 8 \mathrm{H}), 7.05(\mathrm{~d}, J=8.1 \mathrm{~Hz}, 2 \mathrm{H}), 2.62(\mathrm{t}, J=7.6 \mathrm{~Hz} 2 \mathrm{H})$, $2.42(\mathrm{t}, J=7.3 \mathrm{~Hz}, 2 \mathrm{H}), 2.27(\mathrm{~s}, 3 \mathrm{H}), 2.07-1.94(\mathrm{~m}, 2 \mathrm{H}), 1.33(\mathrm{~s}, 9 \mathrm{H})$.

${ }^{13}$ C NMR (125 MHz, CDCl $) \delta 171.10,151.40,148.13,144.34,138.26,137.39,137.28$, 136.43 , 135.18, 134.42, 130.96, 130.85, 129.17, 128.14, 127.96, 127.44, 126.89, 124.77, 124.42 , 123.87, 123.09, 121.64, 121.44, 119.36, 116.43, 116.23, 37.23, 34.68, 31.33, 25.39, 23.84, 21.53.

HRMS (ESI-TOF) m/z: [M+H] $]^{+}$calcd for $\mathrm{C}_{38} \mathrm{H}_{38} \mathrm{~N}_{3} \mathrm{O}_{3} \mathrm{~S}$ 616.2628; Found 616.2633.

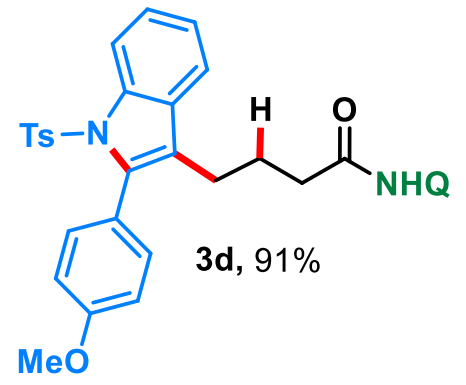

\section{4-(2-(4-methoxyphenyl)-1-tosyl-1H-indol-3-yl)- $N$-(quinolin-8-yl)butanamide (3d)}

$53.7 \mathrm{mg}, 91 \%$ yield, white solid.

$\boldsymbol{R}_{f}: 0.40$ (hexane/ethyl acetate, 6:4 v/v).

${ }^{1}$ H NMR (400 MHz, CDCl3) $\delta 9.64(\mathrm{~s}, 1 \mathrm{H}), 8.79(\mathrm{dd}, J=4.2,1.6 \mathrm{~Hz}, 1 \mathrm{H}), 8.71(\mathrm{dd}, J=6.9$, $1.6 \mathrm{~Hz}, 1 \mathrm{H}), 8.33(\mathrm{~d}, J=8.3 \mathrm{~Hz}, 1 \mathrm{H}), 8.16(\mathrm{dd}, J=8.3,1.4 \mathrm{~Hz}, 1 \mathrm{H}), 7.58-7.44(\mathrm{~m}, 4 \mathrm{H})$, $7.36(\mathrm{~m}, 1 \mathrm{H}), 7.27(\mathrm{~m}, 5 \mathrm{H}), 7.06(\mathrm{~d}, J=8.1 \mathrm{~Hz}, 2 \mathrm{H}), 6.86(\mathrm{~d}, J=8.7 \mathrm{~Hz}, 2 \mathrm{H}), 3.77(\mathrm{~s}, 3 \mathrm{H})$, $2.62(\mathrm{t}, 2 \mathrm{H}), 2.41(\mathrm{t}, J=7.3 \mathrm{~Hz}, 2 \mathrm{H}), 2.28(\mathrm{~s}, 3 \mathrm{H}), 2.03-1.92(\mathrm{~m}, 2 \mathrm{H})$.

${ }^{13}$ C NMR (100 MHz, CDCl $) \delta 171.03,159.70,148.09,144.40,138.22,137.26,136.99$, $136.45,135.28,134.39,132.55,130.82$, 129.22, 127.95, 127.43, 126.78, 124.75, 123.89, $123.38,122.95,121.63,121.41,119.28,116.46,116.20,113.00,55.13,37.15,25.29,23.74$, 21.53 .

HRMS (ESI-TOF) m/z:[M+H] $]^{+}$calcd for $\mathrm{C}_{35} \mathrm{H}_{32} \mathrm{~N}_{3} \mathrm{O}_{4} \mathrm{~S} 590.2108$; Found 590.2119. 


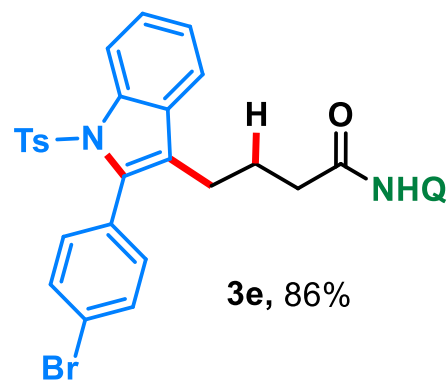

\section{4-(2-(4-bromophenyl)-1-tosyl-1H-indol-3-yl)- $N$-(quinolin-8-yl)butanamide (3e)}

$54.8 \mathrm{mg}, 86 \%$ yield, white solid.

$\boldsymbol{R}_{f}: 0.40$ (hexane/ethyl acetate, $6: 4 \mathrm{v} / \mathrm{v}$ ).

${ }^{1}$ H NMR (400 MHz, CDCl3) $\delta 9.64(\mathrm{~s}, 1 \mathrm{H}), 8.79(\mathrm{dd}, J=4.2,1.6 \mathrm{~Hz}, 1 \mathrm{H}), 8.71(\mathrm{dd}, J=7.2$, $1.5 \mathrm{~Hz}, 1 \mathrm{H}), 8.32$ (d, $J=8.3 \mathrm{~Hz}, 1 \mathrm{H}), 8.16$ (dd, $J=8.3,1.6 \mathrm{~Hz}, 1 \mathrm{H}), 7.62-7.49$ (m, 3H), $7.49-7.42(\mathrm{~m}, 3 \mathrm{H}), 7.42-7.34(\mathrm{~m}, 1 \mathrm{H}), 7.34-7.25(\mathrm{~m}, 3 \mathrm{H}), 7.22(\mathrm{~d}, J=8.4 \mathrm{~Hz}, 2 \mathrm{H}), 7.07$ $(\mathrm{d}, J=8.1 \mathrm{~Hz}, 2 \mathrm{H}), 2.67-2.55(\mathrm{~m}, 2 \mathrm{H}), 2.41(\mathrm{t}, J=7.2 \mathrm{~Hz}, 2 \mathrm{H}), 2.28(\mathrm{~s}, 3 \mathrm{H}), 2.04-1.91$ $(\mathrm{m}, 2 \mathrm{H})$.

${ }^{13}$ C NMR (100 MHz, CDCl3) $\delta 170.79,148.14,144.69,138.24,137.43,136.45,135.70$, $134.95,134.33,132.74,130.84,130.72,130.29$, 129.34, 127.97, 127.46, 126.70, 125.25, $124.15,123.97,123.00,121.66,121.52,119.57,116.43,116.27,37.03,25.28,23.71,21.55$. HRMS (ESI-TOF) m/z: [M+H $]^{+}$calcd for $\mathrm{C}_{34} \mathrm{H}_{29} \mathrm{BrN}_{3} \mathrm{O}_{3} \mathrm{~S}$ 638.1108; Found 638.1108.<smiles>O=C(CCc1c(-c2ccccc2Br)n([123I])c2ccccc12)NO</smiles>

\section{4-(2-(2-bromophenyl)-1-tosyl-1H-indol-3-yl)- $N$-(quinolin-8-yl)butanamide (3f)}

$52.9 \mathrm{mg}, 83 \%$ yield, white solid.

$\boldsymbol{R}_{f}: 0.40$ (hexane/ethyl acetate, $6: 4 \mathrm{v} / \mathrm{v}$ ).

${ }^{1}$ H NMR (400 MHz, CDCl3) $\delta 9.66(\mathrm{~s}, 1 \mathrm{H}), 8.78(\mathrm{dd}, J=4.2,1.6 \mathrm{~Hz}, 1 \mathrm{H}), 8.69$ (dd, $J=6.8$, $2.1 \mathrm{~Hz}, 1 \mathrm{H}), 8.30(\mathrm{~d}, J=8.4 \mathrm{~Hz}, 1 \mathrm{H}), 8.15(\mathrm{dd}, J=8.3,1.6 \mathrm{~Hz}, 1 \mathrm{H}), 7.65(\mathrm{~d}, J=7.6 \mathrm{~Hz}, 1 \mathrm{H})$, 7.59 (dd, $J=7.9,0.9 \mathrm{~Hz}, 1 \mathrm{H}), 7.51-7.43(\mathrm{~m}, 5 \mathrm{H}), 7.42-7.35(\mathrm{~m}, 1 \mathrm{H}), 7.32-7.20(\mathrm{~m}, 4 \mathrm{H})$, $7.12(\mathrm{~d}, J=8.1 \mathrm{~Hz}, 2 \mathrm{H}), 2.72-2.49(\mathrm{~m}, 2 \mathrm{H}), 2.46-2.41(\mathrm{~m}, 2 \mathrm{H}), 2.31(\mathrm{~s}, 3 \mathrm{H}), 2.10-1.91$ $(\mathrm{m}, 2 \mathrm{H})$. 
${ }^{13}$ C NMR (100 MHz, CDCl3) $\delta 171.07,148.10,144.61,138.27,136.53,136.34,135.93$, $134.79,134.42$, 133.22, 132.77, 132.58, 130.34, 129.98, 129.47, 127.90, 127.37, 127.01, 126.59 , 126.47, 125.08, 123.52, 122.84, 121.59, 121.36, 119.72, 116.37, 115.25, 37.31, $24.83,23.87,21.56$.

HRMS (ESI-TOF) m/z: [M+H] $]^{+}$calcd for $\mathrm{C}_{34} \mathrm{H}_{29} \mathrm{BrN}_{3} \mathrm{O}_{3} \mathrm{~S}$ 638.1108; Found 638.1106.

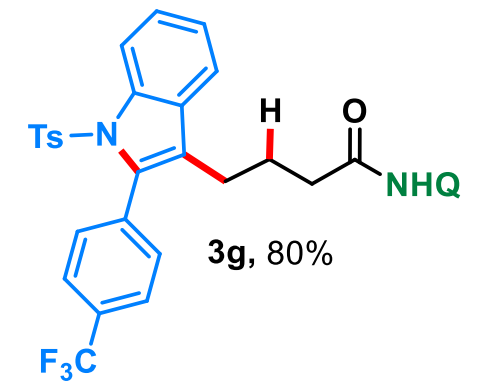

\section{$\underline{N \text {-(quinolin-8-yl)-4-(1-tosyl-2-(4-(trifluoromethyl)phenyl)-1H-indol-3-yl)butanamide }}$}

$\underline{(3 g)}$

$50.2 \mathrm{mg}, 80 \%$ yield, yellow solid.

$\boldsymbol{R}_{f}: 0.35$ (hexane/ethyl acetate, 6:4 v/v).

${ }^{1}$ H NMR (500 MHz, CDCl $) \delta 9.68(\mathrm{~s}, 1 \mathrm{H}), 8.81(\mathrm{dd}, J=4.2,1.6 \mathrm{~Hz}, 1 \mathrm{H}), 8.72(\mathrm{dd}, J=6.9$, $1.9 \mathrm{~Hz}, 1 \mathrm{H}), 8.35(\mathrm{~d}, J=8.4 \mathrm{~Hz}, 1 \mathrm{H}), 8.19(\mathrm{dd}, J=8.3,1.6 \mathrm{~Hz}, 1 \mathrm{H}), 7.63(\mathrm{~d}, J=8.0 \mathrm{~Hz}, 3 \mathrm{H})$, $7.58-7.46(\mathrm{~m}, 5 \mathrm{H}), 7.45-7.40(\mathrm{~m}, 1 \mathrm{H}), 7.32(\mathrm{~m}, 3 \mathrm{H}), 7.10(\mathrm{~d}, J=8.1 \mathrm{~Hz}, 2 \mathrm{H}), 2.65(\mathrm{dd}, J$ $=8.7,6.9 \mathrm{~Hz}, 2 \mathrm{H}), 2.46(\mathrm{t}, J=7.2 \mathrm{~Hz}, 2 \mathrm{H}), 2.31(\mathrm{~s}, 3 \mathrm{H}), 2.07-1.95(\mathrm{~m}, 2 \mathrm{H})$.

${ }^{13}$ C NMR (125 MHz, CDCl3) $\delta$ 170.84, 148.24, 144.91, 138.32, 137.67, 136.57, 135.42, $135.26,134.84,134.36,131.58,130.79,130.42(\mathrm{q}, J=32.5 \mathrm{~Hz}) 130.03,129.47,128.07$, $127.53,126.79,126.03(\mathrm{q}, J=192.8 \mathrm{~Hz}), 125.60,124.83,124.63(\mathrm{q}, J=3.5 \mathrm{~Hz}), 124.38$, 121.76, 121.66, 119.83, 116.54, 116.44, 37.10, 25.41, 23.85, 21.64.

HRMS (ESI-TOF) m/z: [M+H] $]^{+}$calcd for $\mathrm{C}_{35} \mathrm{H}_{29} \mathrm{~F}_{3} \mathrm{~N}_{3} \mathrm{O}_{3} \mathrm{~S}$ 628.1876; Found 628.1877.

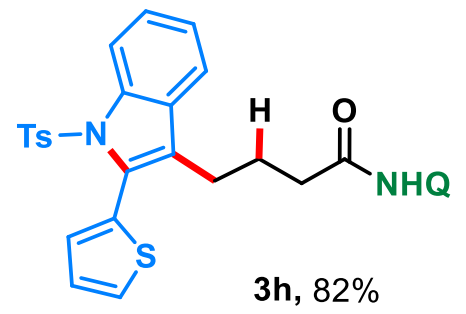

\section{$\underline{N \text {-(quinolin-8-yl)-4-(2-(thiophen-2-yl)-1-tosyl-1H-indol-3-yl)butanamide (3h) }}$}

$46.3 \mathrm{mg}, 82 \%$ yield, white solid.

$\boldsymbol{R}_{f}: 0.40$ (hexane/ethyl acetate, $6: 4 \mathrm{v} / \mathrm{v}$ ). 


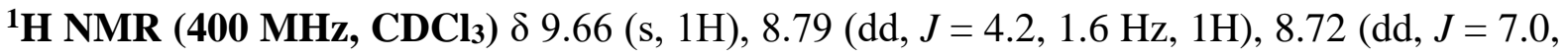
$1.6 \mathrm{~Hz}, 1 \mathrm{H}), 8.33$ (d, $J=8.3 \mathrm{~Hz}, 1 \mathrm{H}), 8.16$ (dd, $J=8.3,1.5 \mathrm{~Hz}, 1 \mathrm{H}), 7.60-7.42(\mathrm{~m}, 4 \mathrm{H})$, $7.38-7.34(\mathrm{~m}, 1 \mathrm{H}), 7.33-7.24(\mathrm{~m}, 4 \mathrm{H}), 7.20(\mathrm{dd}, J=2.9,1.1 \mathrm{~Hz}, 1 \mathrm{H}), 7.16(\mathrm{dd}, J=4.9,1.1$ $\mathrm{Hz}, 1 \mathrm{H}), 7.07(\mathrm{~d}, J=8.1 \mathrm{~Hz}, 2 \mathrm{H}), 2.71-2.59(\mathrm{~m}, 2 \mathrm{H}), 2.42(\mathrm{t}, J=7.3 \mathrm{~Hz}, 2 \mathrm{H}), 2.27(\mathrm{~s}, 3 \mathrm{H})$, $2.04-1.93(\mathrm{~m}, 2 \mathrm{H})$.

${ }^{13}$ C NMR (100 MHz, CDCl $) \delta 171.11,148.24,144.59,138.38,137.44,136.53,135.38$, 134.49 , 132.03, 131.19, 131.00, 130.66, 129.37, 128.05, 127.53, 126.83, 126.46, 125.12, $124.40,123.98,123.91,121.74,121.56,119.45,116.55,116.17,37.28,25.58,24.01,21.63$.

HRMS (ESI-TOF) m/z: [M+H] $]^{+}$calcd for $\mathrm{C}_{32} \mathrm{H}_{28} \mathrm{~N}_{3} \mathrm{O}_{3} \mathrm{~S}_{2}$ 566.1567; Found 566.1568.

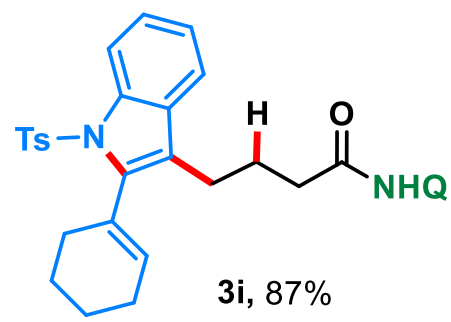

4-(2-(cyclohex-1-en-1-yl)-1-tosyl-1H-indol-3-yl)- $N$-(quinolin-8-yl)butanamide (3i) $49.0 \mathrm{mg}, 87 \%$ yield, white solid.

$\boldsymbol{R}_{f}: 0.40$ (hexane/ethyl acetate, 6:4 v/v).

${ }^{1}$ H NMR (400 MHz, CDCl 3$) \delta 9.74(\mathrm{~s}, 1 \mathrm{H}), 8.85-8.72(\mathrm{~m}, 2 \mathrm{H}), 8.22(\mathrm{~d}, J=8.3 \mathrm{~Hz}, 1 \mathrm{H})$, $8.16(\mathrm{dd}, J=8.3,1.4 \mathrm{~Hz}, 1 \mathrm{H}), 7.58-7.42(\mathrm{~m}, 6 \mathrm{H}), 7.33-7.19(\mathrm{~m}, 2 \mathrm{H}), 7.09(\mathrm{~d}, J=8.1 \mathrm{~Hz}$, $2 \mathrm{H}), 5.56(\mathrm{~s}, 1 \mathrm{H}), 2.70(\mathrm{~m}, 3 \mathrm{H}), 2.52(\mathrm{t}, J=7.3 \mathrm{~Hz}, 2 \mathrm{H}), 2.26(\mathrm{~m}, 3 \mathrm{H}), 2.19(\mathrm{~m}, 2 \mathrm{H}), 2.03(\mathrm{~d}$, $J=12.2 \mathrm{~Hz}, 3 \mathrm{H}), 1.78(\mathrm{~m}, 3 \mathrm{H}), 1.65(\mathrm{~m}, 1 \mathrm{H})$.

${ }^{13}$ C NMR (100 MHz, CDCl 3$) \delta 171.27,148.23,144.41,140.03,138.42,137.03,136.53$, $135.51,134.58,131.31,131.08,130.68,129.32,128.07,127.55,126.83,124.55,123.76$, $121.74,121.55,121.50,119.42,116.52,115.87,37.69,31.02,25.98,25.77,24.15,22.85$, 22.07, 21.61.

HRMS (ESI-TOF) m/z: [M+H] $]^{+}$calcd for $\mathrm{C}_{34} \mathrm{H}_{34} \mathrm{~N}_{3} \mathrm{O}_{3} \mathrm{~S} 564.2315$; Found 564.2318.

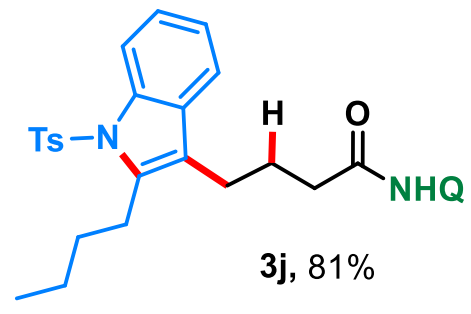

4-(2-butyl-1-tosyl-1H-indol-3-yl)- $N$-(quinolin-8-yl)butanamide (3j) 
$43.7 \mathrm{mg}, 81 \%$ yield, white solid.

$\boldsymbol{R}_{f}: 0.40$ (hexane/ethyl acetate, $6: 4 \mathrm{v} / \mathrm{v}$ ).

${ }^{1}$ H NMR (400 MHz, CDCl $) \delta 9.73(\mathrm{~s}, 1 \mathrm{H}), 8.88-8.73(\mathrm{~m}, 2 \mathrm{H}), 8.18$ (d, J = 8.2 Hz, 2H), $7.60-7.49(\mathrm{~m}, 4 \mathrm{H}), 7.49-7.42(\mathrm{~m}, 2 \mathrm{H}), 7.27-7.19(\mathrm{~m}, 2 \mathrm{H}), 7.14(\mathrm{~d}, J=8.1 \mathrm{~Hz}, 2 \mathrm{H}), 2.97$ $(\mathrm{t}, J=8.0 \mathrm{~Hz}, 2 \mathrm{H}), 2.75(\mathrm{t}, J=7.5 \mathrm{~Hz}, 2 \mathrm{H}), 2.45(\mathrm{t}, J=7.2 \mathrm{~Hz}, 2 \mathrm{H}), 2.23(\mathrm{~s}, 3 \mathrm{H}), 2.05(\mathrm{t}, J=$ $7.4 \mathrm{~Hz}, 2 \mathrm{H}), 1.71-1.63(\mathrm{~m}, 2 \mathrm{H}), 1.42-1.29(\mathrm{~m}, 2 \mathrm{H}), 0.88$ (t, $J=7.3 \mathrm{~Hz}, 3 \mathrm{H})$.

${ }^{13}$ C NMR (100 MHz, CDCl3) $\delta$ 171.14, 148.13, 144.42, 138.39, 138.29, 137.03, 136.48, $134.41,130.98,129.60,127.99,127.46,126.18,124.01,123.59,121.67,121.52,120.84$, $118.64,116.48,115.57,36.95,33.17,26.46,25.34,23.51,22.70,21.44,13.82$.

HRMS (ESI-TOF) m/z: [M+H] $]^{+}$calcd for $\mathrm{C}_{32} \mathrm{H}_{34} \mathrm{~N}_{3} \mathrm{O}_{3} \mathrm{~S} 540.2315$; Found 540.2315.<smiles>O=C(CCCc1c(C2CC2)n([125I])c2ccccc12)NO</smiles>

\section{4-(2-cyclopropyl-1-tosyl-1H-indol-3-yl)- $N$-(quinolin-8-yl)butanamide (3k)}

$45.5 \mathrm{mg}, 87 \%$ yield, white solid.

$\boldsymbol{R}_{f}: 0.40$ (hexane/ethyl acetate, $6: 4 \mathrm{v} / \mathrm{v}$ ).

${ }^{1}$ H NMR (500 MHz, CDCl 3$) \delta 9.74(\mathrm{~s}, 1 \mathrm{H}), 8.88-8.75(\mathrm{~m}, 2 \mathrm{H}), 8.23(\mathrm{~d}, J=8.3 \mathrm{~Hz}, 1 \mathrm{H})$, $8.19(\mathrm{~d}, J=8.2 \mathrm{~Hz}, 1 \mathrm{H}), 7.64(\mathrm{~d}, J=8.3 \mathrm{~Hz}, 2 \mathrm{H}), 7.61-7.51(\mathrm{~m}, 2 \mathrm{H}), 7.48(\mathrm{dd}, J=8.1,3.5$ $\mathrm{Hz}, 2 \mathrm{H}), 7.30(\mathrm{~m}, 1 \mathrm{H}), 7.22(\mathrm{~m}, 1 \mathrm{H}), 7.18(\mathrm{~d}, J=8.2 \mathrm{~Hz}, 2 \mathrm{H}), 2.88(\mathrm{t}, J=7.5 \mathrm{~Hz}, 2 \mathrm{H}), 2.43$ (t, $J=7.3 \mathrm{~Hz}, 2 \mathrm{H}), 2.25(\mathrm{~s}, 3 \mathrm{H}), 2.15-2.06(\mathrm{~m}, 2 \mathrm{H}), 2.04-1.99(\mathrm{~m}, 1 \mathrm{H}), 1.06-0.97$ (m, $2 \mathrm{H}), 0.74-0.65(\mathrm{~m}, 2 \mathrm{H})$.

${ }^{13}$ C NMR (125 MHz, CDCl $) \delta 171.25,148.22,144.48,138.34,138.23,137.18,136.55$, $136.29,134.50,130.82,129.59,128.05,127.53,126.43,124.62,123.59,122.91,121.75$, $121.60,118.91,116.52,115.53,37.01,25.32,23.72,21.52,8.65,8.40$.

HRMS (ESI-TOF) m/z: [M+H] $]^{+}$calcd for $\mathrm{C}_{31} \mathrm{H}_{30} \mathrm{~N}_{3} \mathrm{O}_{3} \mathrm{~S}$ 524.2002; Found 524.2015.<smiles>O=C(CCCc1c(CO)n([12F])c2ccccc12)NO</smiles>

$\underline{\text { 4-(2-(hydroxymethyl)-1-tosyl-1H-indol-3-yl)- } N \text {-(quinolin-8-yl)butanamide (3l) }}$ 
$36.9 \mathrm{mg}, 72 \%$ yield, white solid.

$\boldsymbol{R}_{f}: 0.40$ (hexane/ethyl acetate, $6: 4 \mathrm{v} / \mathrm{v}$ ).

${ }^{1}$ H NMR (400 MHz, CDCl $) \delta 9.79$ (s, 1H), 8.81 (dd, $\left.J=4.1,1.5 \mathrm{~Hz}, 1 \mathrm{H}\right), 8.74(\mathrm{dd}, J=6.4$, $2.4 \mathrm{~Hz}, 1 \mathrm{H}), 8.16(\mathrm{dd}, J=8.3,1.4 \mathrm{~Hz}, 1 \mathrm{H}), 8.06(\mathrm{~d}, J=8.3 \mathrm{~Hz}, 1 \mathrm{H}), 7.73(\mathrm{~d}, J=8.3 \mathrm{~Hz}, 2 \mathrm{H})$, $7.58-7.48(\mathrm{~m}, 3 \mathrm{H}), 7.46(\mathrm{dd}, J=8.2,4.2 \mathrm{~Hz}, 1 \mathrm{H}), 7.33-7.25(\mathrm{~m}, 1 \mathrm{H}), 7.22(\mathrm{t}, J=7.2 \mathrm{~Hz}$, $1 \mathrm{H}), 7.17(\mathrm{~d}, J=8.1 \mathrm{~Hz}, 2 \mathrm{H}), 4.94(\mathrm{~s}, 2 \mathrm{H}), 2.87(\mathrm{t}, J=7.2 \mathrm{~Hz}, 2 \mathrm{H}), 2.54(\mathrm{t}, J=6.9 \mathrm{~Hz}, 2 \mathrm{H})$, $2.27(\mathrm{~s}, 3 \mathrm{H}), 2.21-2.07(\mathrm{~m}, 2 \mathrm{H})$.

${ }^{13}$ C NMR (100 MHz, CDCl3) $\delta 171.51,148.32,144.99,138.42,136.61,136.44,135.89$, $135.68,134.47,129.97,129.94,128.11,127.50,126.56,125.40,123.71,123.00,121.71$, $119.80,116.81,114.82,54.87,36.59,25.55,23.41,21.61$.

HRMS (ESI-TOF) m/z: [M+H] $]^{+}$calcd for $\mathrm{C}_{29} \mathrm{H}_{28} \mathrm{~N}_{3} \mathrm{O}_{4} \mathrm{~S} 514.1795$; Found 514.1805.<smiles>CC(O)Cc1c(CCC(=O)NO)c2ccccc2n1[13CH3]</smiles>

\section{4-(2-(2-hydroxypropyl)-1-tosyl-1H-indol-3-yl)- $N$-(quinolin-8-yl)butanamide (3m)}

$40.0 \mathrm{mg}, 74 \%$ yield, white solid.

$\boldsymbol{R}_{f}: 0.40$ (hexane/ethyl acetate, 6:4 v/v).

${ }^{1}$ H NMR (500 MHz, CDCl3) $\delta 9.72(\mathrm{~s}, 1 \mathrm{H}), 8.84-8.71$ (m, 2H), 8.15 (m, 2H), 7.56 - 7.47 $(\mathrm{m}, 5 \mathrm{H}), 7.46-7.41(\mathrm{~m}, 1 \mathrm{H}), 7.24(\mathrm{t}, J=7.6 \mathrm{~Hz}, 1 \mathrm{H}), 7.20(\mathrm{t}, J=7.4 \mathrm{~Hz}, 1 \mathrm{H}), 7.11(\mathrm{~d}, J=$ $8.0 \mathrm{~Hz}, 2 \mathrm{H}), 4.42-4.31(\mathrm{~m}, 1 \mathrm{H}), 3.27$ (dd, $J=14.5,4.1 \mathrm{~Hz}, 1 \mathrm{H}), 3.09$ (dd, $J=14.5,8.0 \mathrm{~Hz}$, 1H), $2.85-2.75(\mathrm{~m}, 2 \mathrm{H}), 2.47(\mathrm{dd}, J=9.8,4.6 \mathrm{~Hz}, 2 \mathrm{H}), 2.19$ (s, 3H), 2.09 (dd, $J=14.8,7.3$ $\mathrm{Hz}, 2 \mathrm{H}), 1.31(\mathrm{~d}, J=6.2 \mathrm{~Hz}, 3 \mathrm{H})$.

${ }^{13}$ C NMR (125 MHz, CDCl3) $\delta 171.35,148.14,144.64,138.21,137.08,136.51,135.32$, $134.56,134.31,130.91,129.66,127.97,127.38,126.11,124.37,123.79$, 123.47, 121.65, $121.60,118.97,116.57,115.62,68.19,36.85,36.51,25.24,23.68,23.28,21.39$.

HRMS (ESI-TOF) m/z: [M+H] $]^{+}$calcd for $\mathrm{C}_{31} \mathrm{H}_{32} \mathrm{~N}_{3} \mathrm{O}_{4} \mathrm{~S} 542.2108$; Found 542.2128. 
<smiles>CCCCCCCCCCCCC(=O)NO</smiles>

\section{4-(2-((1,3-dioxoisoindolin-2-yl)methyl)-1-tosyl-1H-indol-3-yl)-N-(quinolin-8-}

yl)butanamide (3n)

$51.4 \mathrm{mg}, 80 \%$ yield, white solid.

$\boldsymbol{R}_{\boldsymbol{f}}: 0.30$ (hexane/ethyl acetate, $6: 4 \mathrm{v} / \mathrm{v}$ ).

${ }^{1}$ H NMR (400 MHz, CDCl3) $\delta 9.74(\mathrm{~s}, 1 \mathrm{H}), 8.81-8.70$ (m, 2H), 8.15 (dd, $J=13.3,8.3 \mathrm{~Hz}$, 2H), 7.70 - $7.68(\mathrm{~m}, 2 \mathrm{H}), 7.63-7.56(\mathrm{~m}, 3 \mathrm{H}), 7.54-7.46(\mathrm{~m}, 4 \mathrm{H}), 7.46-7.41(\mathrm{~m}, 1 \mathrm{H}), 7.30$ (t, $J=7.7 \mathrm{~Hz}, 1 \mathrm{H}), 7.23(\mathrm{t}, J=7.5 \mathrm{~Hz}, 1 \mathrm{H}), 7.00(\mathrm{~d}, J=8.1 \mathrm{~Hz}, 2 \mathrm{H}), 5.25(\mathrm{~s}, 2 \mathrm{H}), 3.02-2.91$ (m, 2H), $2.51(\mathrm{t}, J=7.1 \mathrm{~Hz}, 2 \mathrm{H}), 2.18-2.05(\mathrm{~m}, 5 \mathrm{H})$.

${ }^{13}$ C NMR (100 MHz, CDCl $) \delta 171.12,167.66,148.05,144.54,138.07,137.50,136.67$, $135.77,134.35,133.81,131.96,130.18,129.67,128.95,128.01,127.49$, 126.32, 126.00, $125.38,123.82$, 123.15, 121.66, 121.57, 119.62, 116.72, 115.47, 77.36, 37.01, 34.49, 25.13, $23.94,21.47$.

HRMS (ESI-TOF) m/z: [M+H $]^{+}$calcd for $\mathrm{C}_{37} \mathrm{H}_{31} \mathrm{~N}_{4} \mathrm{O}_{5} \mathrm{~S}$ 643.2010; Found 643.2015

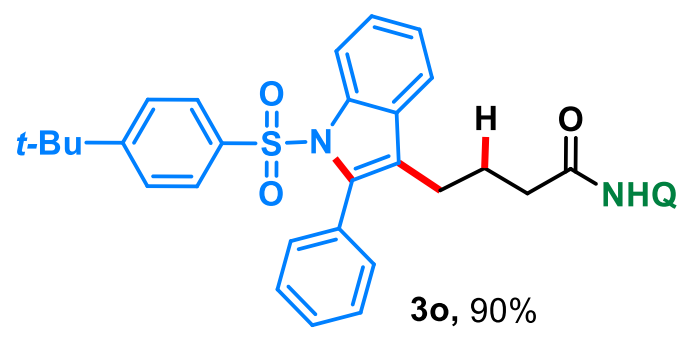

4-(1-((4-(tert-butyl)phenyl)sulfonyl)-2-phenyl-1H-indol-3-yl)- $N$-(quinolin-8-

yl)butanamide (3o)

$54.2 \mathrm{mg}, 90 \%$ yield, white solid.

$\boldsymbol{R}_{f}: 0.40$ (hexane/ethyl acetate, 6:4 v/v).

${ }^{1}$ H NMR (500 MHz, CDCl3) $\delta 9.67(\mathrm{~s}, 1 \mathrm{H}), 8.80(\mathrm{dd}, J=4.2,1.6 \mathrm{~Hz}, 1 \mathrm{H}), 8.74(\mathrm{~d}, J=7.1$ $\mathrm{Hz}, 1 \mathrm{H}), 8.37(\mathrm{~d}, J=8.4 \mathrm{~Hz}, 1 \mathrm{H}), 8.18(\mathrm{dd}, J=8.2,1.4 \mathrm{~Hz}, 1 \mathrm{H}), 7.63(\mathrm{~d}, J=7.7 \mathrm{~Hz}, 1 \mathrm{H})$, $7.57-7.50(\mathrm{~m}, 2 \mathrm{H}), 7.47(\mathrm{dd}, J=8.2,4.2 \mathrm{~Hz}, 1 \mathrm{H}), 7.43-7.38(\mathrm{~m}, 2 \mathrm{H}), 7.36(\mathrm{t}, J=8.6 \mathrm{~Hz}$, 
6H), $7.32(\mathrm{dd}, J=11.2,4.6 \mathrm{~Hz}, 3 \mathrm{H}), 2.70-2.61(\mathrm{~m}, 2 \mathrm{H}), 2.44(\mathrm{t}, J=7.3 \mathrm{~Hz}, 2 \mathrm{H}), 2.04-$ $1.96(\mathrm{~m}, 2 \mathrm{H}), 1.25(\mathrm{~s}, 9 \mathrm{H})$.

${ }^{13}$ C NMR (125 MHz, CDCl $) \delta 171.10,157.44,148.19,138.35,137.31,137.00,136.48$, 135.46 , 134.48, 131.40, 131.38, 130.70, 128.59, 128.00, 127.60, 127.47, 126.78, 125.76, $124.99,123.92$, 123.09, 121.70, 121.50, 119.50, 116.50, 116.12, 37.35, 35.22, 31.02, 25.48, 23.85 .

HRMS (ESI-TOF) m/z: [M+H] $]^{+}$calcd for $\mathrm{C}_{37} \mathrm{H}_{36} \mathrm{~N}_{3} \mathrm{O}_{3} \mathrm{~S}$ 602.2472; Found 602.2496.

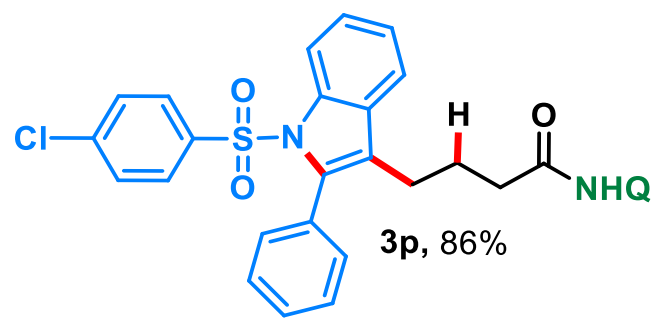

4-(1-((4-chlorophenyl)sulfonyl)-2-phenyl-1H-indol-3-yl)- $N$-(quinolin-8-yl)butanamide $\underline{(3 p)}$

$49.9 \mathrm{mg}, 86 \%$ yield, white solid.

$\boldsymbol{R}_{f}: 0.40$ (hexane/ethyl acetate, $6: 4 \mathrm{v} / \mathrm{v}$ ).

${ }^{1}$ H NMR (500 MHz, CDCl 3$) \delta 9.67(\mathrm{~s}, 1 \mathrm{H}), 8.81(\mathrm{dd}, J=4.2,1.7 \mathrm{~Hz}, 1 \mathrm{H}), 8.74(\mathrm{dd}, J=7.2$, $1.5 \mathrm{~Hz}, 1 \mathrm{H}), 8.34(\mathrm{~d}, J=8.3 \mathrm{~Hz}, 1 \mathrm{H}), 8.18(\mathrm{dd}, J=8.3,1.6 \mathrm{~Hz}, 1 \mathrm{H}), 7.64(\mathrm{~d}, J=7.4 \mathrm{~Hz}, 1 \mathrm{H})$, $7.57-7.50(\mathrm{~m}, 2 \mathrm{H}), 7.47(\mathrm{dd}, J=8.3,4.2 \mathrm{~Hz}, 1 \mathrm{H}), 7.43-7.40(\mathrm{~m}, 1 \mathrm{H}), 7.39-7.37(\mathrm{~m}, 5 \mathrm{H})$, $7.36(\mathrm{t}, J=2.1 \mathrm{~Hz}, 2 \mathrm{H}), 7.35-7.31(\mathrm{~m}, 1 \mathrm{H}), 7.29-7.25(\mathrm{~m}, 2 \mathrm{H}), 2.72-2.62(\mathrm{~m}, 2 \mathrm{H}), 2.45$ $(\mathrm{t}, J=7.3 \mathrm{~Hz}, 2 \mathrm{H}), 2.06-1.96(\mathrm{~m}, 2 \mathrm{H})$.

${ }^{13}$ C NMR (125 MHz, CDCl $) \delta 170.96,148.19,140.18,138.32,137.29,136.86,136.48$, 136.42 , 134.45, 131.29, 131.11, 130.96, 129.05, 128.77, 128.28, 128.00, 127.74, 127.48, $125.29,124.41,124.04,121.70,121.52,119.76,116.50,116.20,37.25,25.38,23.86$.

HRMS (ESI-TOF) m/z: [M+H] $]^{+}$calcd for $\mathrm{C}_{33} \mathrm{H}_{27} \mathrm{ClN}_{3} \mathrm{O}_{3} \mathrm{~S} 580.1456$; Found 580.1460.<smiles></smiles>

4-(1-((2,4-difluorophenyl)sulfonyl)-2-phenyl-1H-indol-3-yl)- $N$-(quinolin-8yl)butanamide (3q) 
$51.2 \mathrm{mg}, 88 \%$ yield, yellow solid.

$\boldsymbol{R}_{f}: 0.40$ (hexane/ethyl acetate, 6:4 v/v).

${ }^{1}$ H NMR (500 MHz, CDCl $) \delta 9.69$ (s, 1H), 8.80 (dd, $\left.J=4.2,1.6 \mathrm{~Hz}, 1 \mathrm{H}\right), 8.74(\mathrm{dd}, J=7.2$, $1.5 \mathrm{~Hz}, 1 \mathrm{H}), 8.23-8.16(\mathrm{~m}, 2 \mathrm{H}), 7.72-7.65(\mathrm{~m}, 1 \mathrm{H}), 7.58-7.50(\mathrm{~m}, 2 \mathrm{H}), 7.48(\mathrm{dd}, J=8.3$, $4.2 \mathrm{~Hz}, 1 \mathrm{H}), 7.40-7.28(\mathrm{~m}, 6 \mathrm{H}), 7.27-7.22(\mathrm{~m}, 2 \mathrm{H}), 6.80-6.72(\mathrm{~m}, 2 \mathrm{H}), 2.71-2.64(\mathrm{~m}$, $2 \mathrm{H}), 2.49(\mathrm{t}, J=7.4 \mathrm{~Hz}, 2 \mathrm{H}), 2.12-2.02(\mathrm{~m}, 2 \mathrm{H})$.

${ }^{13}$ C NMR (125 MHz, CDCl3) $\delta 171.08,166.18(\mathrm{dd}, J=259.3,11.2 \mathrm{~Hz}), 160.21(\mathrm{dd}, J=$ 262.2, $13.0 \mathrm{~Hz}), 148.19,138.33,137.11,136.50$ (d, $J=4.9 \mathrm{~Hz}), 134.46,132.55$ (d, $J=10.3$ Hz), 131.55, 130.69, 130.09, 128.72, 128.02, 127.66, 127.50, 125.06, 124.00, 122.94, 121.71, 121.54, 119.63, 116.55, 115.85, 111.54 (dd, $J=22.1,3.6 \mathrm{~Hz}), 105.64$ (dd, $J=25.8,24.8 \mathrm{~Hz})$, $37.38,25.50,23.83$.

HRMS (ESI-TOF) m/z: [M+H $]^{+}$calcd for $\mathrm{C}_{33} \mathrm{H}_{26} \mathrm{~F}_{2} \mathrm{~N}_{3} \mathrm{O}_{3} \mathrm{~S}$ 582.1657; Found 582.1665.

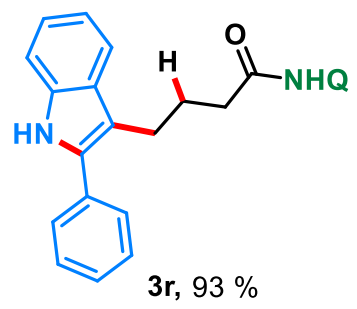

\section{4-(2-phenyl-1H-indol-3-yl)-N-(quinolin-8-yl)butanamide (3r)}

$37.7 \mathrm{mg}, 93 \%$ yield, white solid.

$\boldsymbol{R}_{f}: 0.40$ (hexane/ethyl acetate, $7: 3 \mathrm{v} / \mathrm{v}$ ).

${ }^{1}$ H NMR (400 MHz, CDCl3) $\delta 9.76$ (s, 1H), $8.80-8.77$ (m, 2H), 8.22 (s, 1H), 8.16 (dd, $J=$ 8.3, 1.3 Hz, 1H), 7.73 (d, $J=7.8 \mathrm{~Hz}, 1 \mathrm{H}), 7.56-7.49$ (m, 4H), $7.44(\mathrm{dd}, J=8.3,4.2 \mathrm{~Hz}, 1 \mathrm{H})$, $7.36(\mathrm{~m}, 3 \mathrm{H}), 7.28-7.19(\mathrm{~m}, 2 \mathrm{H}), 7.15(\mathrm{t}, J=7.4 \mathrm{~Hz}, 1 \mathrm{H}), 3.12-3.01(\mathrm{~m}, 2 \mathrm{H}), 2.62(\mathrm{t}, J=$ $7.3 \mathrm{~Hz}, 2 \mathrm{H}), 2.34-2.19(\mathrm{~m}, 2 \mathrm{H})$.

${ }^{13}$ C NMR (100 MHz, CDCl $) \delta 171.71,148.03,138.24,136.62,136.03,134.69,134.54$, $133.28,129.31,128.88,128.07,128.04,127.59,122.32,121.61,121.45,119.70,119.43$, $116.75,112.72,110.92,37.86,26.64,24.12$.

HRMS (ESI-TOF) m/z: [M+H] $]^{+}$calcd for $\mathrm{C}_{27} \mathrm{H}_{24} \mathrm{~N}_{3} \mathrm{O}$ 406.1914; Found 406.1913. 


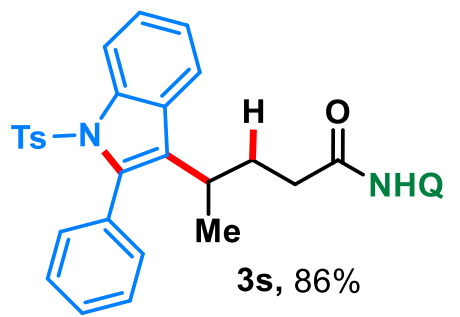

\section{(R)-4-(2-phenyl-1-tosyl-1H-indol-3-yl)-N-(quinolin-8-yl)pentanamide (3s)}

$49.3 \mathrm{mg}, 86 \%$ yield, white solid.

$\boldsymbol{R}_{f}: 0.50$ (hexane/ethyl acetate, $6: 4 \mathrm{v} / \mathrm{v}$ ).

${ }^{1}$ H NMR (400 MHz, CDCl3) $\delta 9.54(\mathrm{~s}, 1 \mathrm{H}), 8.78(\mathrm{dd}, J=4.2,1.6 \mathrm{~Hz}, 1 \mathrm{H}), 8.63(\mathrm{dd}, J=5.7$, $3.3 \mathrm{~Hz}, 1 \mathrm{H}), 8.36(\mathrm{~d}, J=8.4 \mathrm{~Hz}, 1 \mathrm{H}), 8.16(\mathrm{dd}, J=8.3,1.6 \mathrm{~Hz}, 1 \mathrm{H}), 7.71(\mathrm{~d}, J=7.7 \mathrm{~Hz}, 1 \mathrm{H})$, $7.50(\mathrm{~d}, J=2.4 \mathrm{~Hz}, 1 \mathrm{H}), 7.49(\mathrm{~s}, 1 \mathrm{H}), 7.45(\mathrm{dd}, J=8.3,4.2 \mathrm{~Hz}, 1 \mathrm{H}), 7.42-7.34(\mathrm{~m}, 2 \mathrm{H})$, $7.33-7.25(\mathrm{~m}, 7 \mathrm{H}), 7.08(\mathrm{~d}, J=8.1 \mathrm{~Hz}, 2 \mathrm{H}), 2.92-2.77(\mathrm{~m}, 1 \mathrm{H}), 2.26(\mathrm{~s}, 3 \mathrm{H}), 2.25-2.13$ $(\mathrm{m}, 4 \mathrm{H}), 1.31(\mathrm{~d}, J=7.1 \mathrm{~Hz}, 3 \mathrm{H})$.

${ }^{13}$ C NMR (100 MHz, CDCl3) $\delta$ 171.16, 148.19, 144.59, 138.29, 137.77, 136.84, 136.48, 135.42 , 134.43, 131.45, 129.34, 129.07, 128.59, 127.97, 127.52, 127.45, 126.86, 126.63, $124.75,123.69$, 121.69, 121.45, 120.75, 116.46, 116.33, 77.48, 77.16, 76.84, 36.27, 31.14, $31.01,21.59,20.87$.

HRMS (ESI-TOF) m/z: [M+H] $]^{+}$calcd for $\mathrm{C}_{35} \mathrm{H}_{32} \mathrm{~N}_{3} \mathrm{O}_{3} \mathrm{~S} 574.2159$; Found 574.2150.

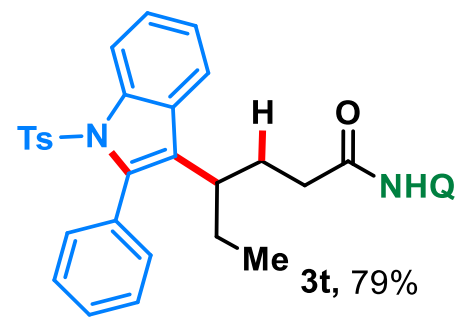

\section{4-(2-phenyl-1-tosyl-1H-indol-3-yl)- $N$-(quinolin-8-yl)hexanamide (3t)}

$46.4 \mathrm{mg}, 79 \%$ yield, white solid.

$\boldsymbol{R}_{f}: 0.50$ (hexane/ethyl acetate, 6:4 v/v).

${ }^{1}$ H NMR (400 MHz, CDCl 3$) \delta 9.51(\mathrm{~s}, 1 \mathrm{H}), 8.78(\mathrm{dd}, J=4.2,1.7 \mathrm{~Hz}, 1 \mathrm{H}), 8.67-8.57(\mathrm{~m}$, $1 \mathrm{H}), 8.36(\mathrm{~d}, J=8.4 \mathrm{~Hz}, 1 \mathrm{H}), 8.16(\mathrm{dd}, J=8.3,1.6 \mathrm{~Hz}, 1 \mathrm{H}), 7.67(\mathrm{~d}, J=7.8 \mathrm{~Hz}, 1 \mathrm{H}), 7.53-$ $7.48(\mathrm{~m}, 2 \mathrm{H}), 7.45$ (dd, $J=8.3,4.2 \mathrm{~Hz}, 1 \mathrm{H}), 7.39$ - 7.34 (m, 1H), 7.35 - 7.29 (m, 4H), 7.29 $7.23(\mathrm{~m}, 3 \mathrm{H}), 7.13(\mathrm{t}, J=7.2 \mathrm{~Hz}, 1 \mathrm{H}), 7.08(\mathrm{~d}, J=8.1 \mathrm{~Hz}, 2 \mathrm{H}), 2.73-2.57(\mathrm{~m}, 1 \mathrm{H}), 2.24(\mathrm{~s}$, $3 \mathrm{H}), 2.18(\mathrm{~d}, J=3.1 \mathrm{~Hz}, 4 \mathrm{H}), 1.86-1.69(\mathrm{~m}, 2 \mathrm{H}), 0.63(\mathrm{t}, J=7.4 \mathrm{~Hz}, 3 \mathrm{H})$.

${ }^{13}$ C NMR (100 MHz, CDCl3) $\delta 171.21,148.18,144.62,138.30,138.14,138.00,136.48$, $135.18,134.44,131.73,131.53,131.37,129.30$, 129.25, 128.50, 127.98, 127.46, 126.81, 
$125.28,124.83,123.82,121.69,121.44,120.81,116.54,116.45,38.18,36.08,29.90,27.72$, $21.56,12.57$.

HRMS (ESI-TOF) m/z: [M+H] $]^{+}$calcd for $\mathrm{C}_{36} \mathrm{H}_{34} \mathrm{~N}_{3} \mathrm{O}_{3} \mathrm{~S} 588.2315$; Found 588.2324.<smiles>O=C(CCC(c1ccccc1)c1c(-c2ccccc2)n([125I])c2ccccc12)NO</smiles>

\section{4-phenyl-4-(2-phenyl-1-tosyl-1H-indol-3-yl)- $N$-(quinolin-8-yl)butanamide (3u)}

$56.6 \mathrm{mg}, 89 \%$ yield, white solid.

$\boldsymbol{R}_{f}: 0.40$ (hexane/ethyl acetate, $6: 4 \mathrm{v} / \mathrm{v}$ ).

${ }^{1}$ H NMR (500 MHz, CDCl3) $\delta 9.55(\mathrm{~s}, 1 \mathrm{H}), 8.81(\mathrm{~d}, J=3.7 \mathrm{~Hz}, 1 \mathrm{H}), 8.74-8.57(\mathrm{~m}, 1 \mathrm{H})$, $8.41(\mathrm{~d}, J=8.4 \mathrm{~Hz}, 1 \mathrm{H}), 8.19(\mathrm{~d}, J=8.2 \mathrm{~Hz}, 1 \mathrm{H}), 7.53(\mathrm{~d}, J=4.7 \mathrm{~Hz}, 2 \mathrm{H}), 7.51-7.42$ (m, 3H), $7.41-7.28(\mathrm{~m}, 5 \mathrm{H}), 7.24-7.19(\mathrm{~m}, 5 \mathrm{H}), 7.14-7.10(\mathrm{~m}, 5 \mathrm{H}), 4.18(\mathrm{dd}, J=10.5,5.4$ $\mathrm{Hz}, 1 \mathrm{H}), 2.77$ (dd, $J=13.5,5.9 \mathrm{~Hz}, 1 \mathrm{H}), 2.67-2.49$ (m, 1H), $2.41-2.25$ (m, 5H).

${ }^{13}$ C NMR (125 MHz, CDCl $) \delta 170.76,148.18,144.66,142.84,138.59,138.26,138.10$, $136.43,134.92$, 134.36, 131.99, 131.14, 130.55, 129.63, 129.30, 128.71, 128.34, 127.93, $127.61,127.45,127.40,126.83,126.26,124.99,124.95,124.14,121.68,121.46,121.16$, $116.69,116.45,40.40,35.91,28.52,21.59$.

HRMS (ESI-TOF) m/z: $[\mathrm{M}+\mathrm{H}]^{+}$calcd for $\mathrm{C}_{40} \mathrm{H}_{34} \mathrm{~N}_{3} \mathrm{O}_{3} \mathrm{~S}$ 636.2315; Found 636.2320.

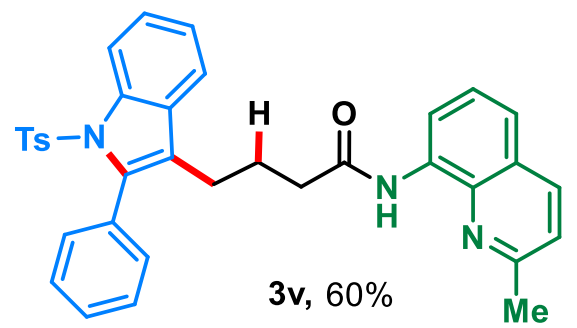

\section{$\underline{N \text {-(2-methylquinolin-8-yl)-4-(2-phenyl-1-tosyl-1H-indol-3-yl)butanamide (3v) }}$}

$34.4 \mathrm{mg}, 60 \%$ yield, white solid.

$\boldsymbol{R}_{f}: 0.40$ (hexane/ethyl acetate, $6: 4 \mathrm{v} / \mathrm{v}$ ).

${ }^{1}$ H NMR (500 MHz, CDCl $) \delta 9.72(\mathrm{~s}, 1 \mathrm{H}), 8.81-8.58(\mathrm{~m}, 1 \mathrm{H}), 8.37(\mathrm{~d}, J=8.3 \mathrm{~Hz}, 1 \mathrm{H})$, $8.05(\mathrm{~d}, J=8.4 \mathrm{~Hz}, 1 \mathrm{H}), 7.62(\mathrm{~d}, J=7.7 \mathrm{~Hz}, 1 \mathrm{H}), 7.47(\mathrm{~d}, J=4.4 \mathrm{~Hz}, 2 \mathrm{H}), 7.43-7.36(\mathrm{~m}$, 6H), $7.36-7.27(\mathrm{~m}, 4 \mathrm{H}), 7.09(\mathrm{~d}, J=8.1 \mathrm{~Hz}, 2 \mathrm{H}), 2.75(\mathrm{~s}, 3 \mathrm{H}), 2.70-2.61(\mathrm{~m}, 2 \mathrm{H}), 2.44(\mathrm{t}$, $J=7.4 \mathrm{~Hz}, 2 \mathrm{H}), 2.29(\mathrm{~s}, 3 \mathrm{H}), 2.06-1.95(\mathrm{~m}, 2 \mathrm{H})$. 
${ }^{13}$ C NMR (125 MHz, CDCl $) \delta 170.98,157.23,144.56,137.69,137.44,137.08,136.55$, $135.24,133.87,131.45,131.35,130.90,129.33$, 128.60, 127.61, 126.87, 126.41, 126.12, $125.03,124.07,123.52$, 122.51, 121.26, 119.53, 116.40, 116.29, 37.37, 25.49, 25.37, 23.88, 21.60 .

HRMS (ESI-TOF) m/z: [M+H] $]^{+}$calcd for $\mathrm{C}_{35} \mathrm{H}_{32} \mathrm{~N}_{3} \mathrm{O}_{3} \mathrm{~S} 574.2159$; Found 574.2165.<smiles>O=C(NO)c1ccccc1CCc1c(-c2ccccc2)n([AsH3])c2ccccc12</smiles>

\section{$\underline{\text { 2-(2-(2-phenyl-1-tosyl-1H-indol-3-yl)ethyl)- } N \text {-(quinolin-8-yl)benzamide (3w) }}$}

$18.7 \mathrm{mg}, 30 \%$ yield, yellow liquid.

$\boldsymbol{R}_{f}: 0.40$ (hexane/ethyl acetate, $9: 1 \mathrm{v} / \mathrm{v}$ ).

${ }^{1}$ H NMR (400 MHz, CDCl $) \delta 10.25(\mathrm{~s}, 1 \mathrm{H}), 8.95(\mathrm{~d}, J=6.8 \mathrm{~Hz}, 1 \mathrm{H}), 8.58(\mathrm{dd}, J=4.2,1.7$ $\mathrm{Hz}, 1 \mathrm{H}), 8.31(\mathrm{~d}, J=8.4 \mathrm{~Hz}, 1 \mathrm{H}), 8.17(\mathrm{dd}, J=8.3,1.6 \mathrm{~Hz}, 1 \mathrm{H}), 7.76(\mathrm{~d}, J=7.9 \mathrm{~Hz}, 1 \mathrm{H})$, $7.70(\mathrm{dd}, J=8.9,7.6 \mathrm{~Hz}, 1 \mathrm{H}), 7.64-7.51(\mathrm{~m}, 4 \mathrm{H}), 7.43$ (dd, $J=5.0,2.0 \mathrm{~Hz}, 3 \mathrm{H}), 7.39$ (dd, $J=7.1,3.0 \mathrm{~Hz}, 2 \mathrm{H}), 7.37-7.35(\mathrm{~m}, 1 \mathrm{H}), 7.34-7.30(\mathrm{~m}, 1 \mathrm{H}), 7.26(\mathrm{~d}, J=1.4 \mathrm{~Hz}, 2 \mathrm{H}), 7.00$ $(\mathrm{d}, J=8.0 \mathrm{~Hz}, 2 \mathrm{H}), 6.97-6.92(\mathrm{~m}, 1 \mathrm{H}), 6.82(\mathrm{~m}, 1 \mathrm{H}), 2.26(\mathrm{~s}, 2 \mathrm{H}), 2.17(\mathrm{~s}, 5 \mathrm{H})$.

${ }^{13}$ C NMR (100 MHz, CDCl3) $\delta 167.87,148.38,144.83,139.34,138.71,137.69,136.55$, $136.43,135.73,134.79,132.03,131.12,130.72,129.49$, 129.07, 128.78, 128.13, 128.08, $127.95,127.63$, 127.58, 126.94, 126.08, 125.21, 124.44, 123.48, 122.07, 121.80, 121.39, $120.97,116.83,116.24,31.07,21.66$.

HRMS (ESI-TOF) m/z: [M+H] $]^{+}$calcd for $\mathrm{C}_{39} \mathrm{H}_{32} \mathrm{~N}_{3} \mathrm{O}_{3} \mathrm{~S}$ 622.2159; Found 622.2135.<smiles>Cc1ccc2c(c1)C(c1ccccc1)=C(CCC(=O)NO)CO2</smiles>

$5 a, 78 \%$

\section{4-(6-methyl-4-phenyl-2H-chromen-3-yl)- $N$-(quinolin-8-yl)butanamide (5a)}

$33.9 \mathrm{mg}, 78 \%$ yield, yellow sticky liquid.

$\boldsymbol{R}_{f}: 0.50$ (hexane/ethyl acetate, $8: 2 \mathrm{v} / \mathrm{v}$ ). 
${ }^{1}$ H NMR (500 MHz, CDCl 3 ) $\delta 9.73(\mathrm{~s}, 1 \mathrm{H}), 8.81(\mathrm{dd}, J=4.2,1.5 \mathrm{~Hz}, 1 \mathrm{H}), 8.74(\mathrm{~d}, J=7.3$ $\mathrm{Hz}, 1 \mathrm{H}), 8.17(\mathrm{dd}, J=8.3,1.4 \mathrm{~Hz}, 1 \mathrm{H}), 7.58-7.50(\mathrm{~m}, 2 \mathrm{H}), 7.47$ (dd, $J=8.2,4.2 \mathrm{~Hz}, 1 \mathrm{H})$, $7.37(\mathrm{t}, J=7.3 \mathrm{~Hz}, 2 \mathrm{H}), 7.30(\mathrm{~m}, 1 \mathrm{H}), 7.25-7.19(\mathrm{~m}, 2 \mathrm{H}), 6.91(\mathrm{~d}, J=8.1 \mathrm{~Hz}, 1 \mathrm{H}), 6.79$ (d, $J=8.1 \mathrm{~Hz}, 1 \mathrm{H}), 6.41(\mathrm{~s}, 1 \mathrm{H}), 4.85(\mathrm{~s}, 2 \mathrm{H}), 2.49(\mathrm{t}, J=7.5 \mathrm{~Hz}, 2 \mathrm{H}), 2.18(\mathrm{dd}, J=13.5,5.3$ $\mathrm{Hz}, 2 \mathrm{H}), 2.14$ (d, $J=7.6 \mathrm{~Hz}, 3 \mathrm{H}), 1.97-1.88(\mathrm{~m}, 2 \mathrm{H})$.

${ }^{13}$ C NMR (125 MHz, CDCl $) \delta 171.06,151.18,148.21,138.37,136.96,136.48,134.46$, $132.23,130.47,130.28,129.95,128.78,128.57,128.01,127.50,127.31,126.36,125.10$, $121.69,121.54,116.58,115.35,68.12,37.55,30.68,23.73,20.79$.

HRMS (ESI-TOF) m/z: [M+H $]^{+}$calcd for $\mathrm{C}_{29} \mathrm{H}_{27} \mathrm{~N}_{2} \mathrm{O}_{2}$ 435.2067; Found 435.2070.<smiles>Cc1ccc2c(c1)C(c1ccccc1C)=C(CCC(=O)NO)CO2</smiles>

4-(6-methyl-4-(o-tolyl)-2H-chromen-3-yl)- $N$-(quinolin-8-yl)butanamide (5b)

$31.4 \mathrm{mg}, 70 \%$ yield, yellow sticky liquid.

$\boldsymbol{R}_{f}: 0.50$ (hexane/ethyl acetate, $8: 2 \mathrm{v} / \mathrm{v}$ ).

${ }^{1}$ H NMR (400 MHz, CDCl3) $\delta 9.72(\mathrm{~s}, 1 \mathrm{H}), 8.79(\mathrm{~d}, J=4.1 \mathrm{~Hz}, 1 \mathrm{H}), 8.72(\mathrm{~d}, J=7.0 \mathrm{~Hz}$, $1 \mathrm{H}), 8.16(\mathrm{~d}, J=8.2 \mathrm{~Hz}, 1 \mathrm{H}), 7.56-7.48(\mathrm{~m}, 2 \mathrm{H}), 7.45(\mathrm{dd}, J=8.2,4.2 \mathrm{~Hz}, 1 \mathrm{H}), 7.26-7.14$ (m, 3H), $7.10(\mathrm{~d}, J=7.4 \mathrm{~Hz}, 1 \mathrm{H}), 6.88(\mathrm{~d}, J=8.1 \mathrm{~Hz}, 1 \mathrm{H}), 6.78(\mathrm{~d}, J=8.1 \mathrm{~Hz}, 1 \mathrm{H}), 6.24(\mathrm{~s}$, $1 \mathrm{H}), 4.97-4.69(\mathrm{~m}, 2 \mathrm{H}), 2.54-2.38(\mathrm{~m}, 2 \mathrm{H}), 2.12(\mathrm{~s}, 3 \mathrm{H}), 2.11(\mathrm{~s}, 3 \mathrm{H}), 2.09-1.95(\mathrm{~m}, 2 \mathrm{H})$, $1.95-1.82(\mathrm{~m}, 2 \mathrm{H})$.

${ }^{13}$ C NMR (100 MHz, CDCl $) \delta 171.06,151.14,148.20,138.37,136.96,136.51,136.23$, $134.46,131.42$, 130.60, 130.32, 130.19, 128.78, 128.03, 127.63, 127.52, 126.00, 125.76, $124.38,121.70,121.55,116.60,115.36,67.93,37.71,30.63,23.44,20.80,19.62$.

HRMS (ESI-TOF) m/z: [M+H] $]^{+}$calcd for $\mathrm{C}_{30} \mathrm{H}_{29} \mathrm{~N}_{2} \mathrm{O}_{2} 449.2224$; Found 449.2237.<smiles>Cc1ccc2c(c1)C(c1ccc([N+](=O)[O-])cc1)=C(CCC(=O)NO)CO2</smiles>

4-(6-methyl-4-(4-nitrophenyl)-2H-chromen-3-yl)- $N$-(quinolin-8-yl)butanamide (5c) 
$36.0 \mathrm{mg}, 75 \%$ yield, yellow solid.

$\boldsymbol{R}_{\boldsymbol{f}}: 0.50$ (hexane/ethyl acetate, $7: 3 \mathrm{v} / \mathrm{v}$ ).

${ }^{1}$ H NMR (400 MHz, CDCl $) \delta 9.67(\mathrm{~s}, 1 \mathrm{H}), 8.77$ (dd, $\left.J=4.2,1.6 \mathrm{~Hz}, 1 \mathrm{H}\right), 8.65$ (dd, $J=5.4$, $3.5 \mathrm{~Hz}, 1 \mathrm{H}), 8.16(\mathrm{dd}, J=8.3,1.6 \mathrm{~Hz}, 1 \mathrm{H}), 8.14-8.08(\mathrm{~m}, 2 \mathrm{H}), 7.50(\mathrm{dd}, J=6.9,3.4 \mathrm{~Hz}$, 2H), $7.45(\mathrm{dd}, J=8.3,4.2 \mathrm{~Hz}, 1 \mathrm{H}), 7.39-7.31(\mathrm{~m}, 2 \mathrm{H}), 6.91(\mathrm{dd}, J=8.1,1.5 \mathrm{~Hz}, 1 \mathrm{H}), 6.78$ (d, $J=8.1 \mathrm{~Hz}, 1 \mathrm{H}), 6.23(\mathrm{~d}, J=1.5 \mathrm{~Hz}, 1 \mathrm{H}), 4.81(\mathrm{~s}, 2 \mathrm{H}), 2.48$ (t, $J=7.1 \mathrm{~Hz}, 2 \mathrm{H}), 2.22-$ $2.12(\mathrm{~m}, 2 \mathrm{H}), 2.11(\mathrm{~s}, 3 \mathrm{H}), 1.96-1.86(\mathrm{~m}, 2 \mathrm{H})$.

${ }^{13}$ C NMR (100 MHz, CDCl $) \delta 170.57,151.11,148.22,147.18,144.19,138.23,136.58$, $134.21,131.62,131.04,130.76,130.62,129.39,128.03,127.47,125.85,124.02,123.84$, $121.75,121.72,116.51,115.70,67.87,37.08,30.48,23.52,20.72$.

HRMS (ESI-TOF) m/z: [M+H $]^{+}$calcd for $\mathrm{C}_{29} \mathrm{H}_{26} \mathrm{~N}_{3} \mathrm{O}_{4} 480.1918$; Found 480.1909.<smiles>Cc1ccc2c(c1)C(c1cccc3ccccc13)=C(CCC(=O)NO)CO2</smiles>

4-(6-methyl-4-(naphthalen-1-yl)-2H-chromen-3-yl)- $N$-(quinolin-8-yl)butanamide (5d)

$40.2 \mathrm{mg}, 83 \%$ yield, brown sticky liquid.

$\boldsymbol{R}_{\boldsymbol{f}}: 0.50$ (hexane/ethyl acetate, $8: 2 \mathrm{v} / \mathrm{v}$ ).

${ }^{1}$ H NMR (400 MHz, CDCl $) \delta 9.61(\mathrm{~s}, 1 \mathrm{H}), 8.76(\mathrm{dd}, J=4.2,1.6 \mathrm{~Hz}, 1 \mathrm{H}), 8.69$ (dd, $J=7.1$, $1.4 \mathrm{~Hz}, 1 \mathrm{H}), 8.13(\mathrm{dd}, J=8.3,1.6 \mathrm{~Hz}, 1 \mathrm{H}), 7.85(\mathrm{dd}, J=9.8,4.4 \mathrm{~Hz}, 2 \mathrm{H}), 7.79$ (d, $J=8.1 \mathrm{~Hz}$, 1H), $7.55-7.46(\mathrm{~m}, 3 \mathrm{H}), 7.45-7.34(\mathrm{~m}, 4 \mathrm{H}), 6.93-6.78(\mathrm{~m}, 2 \mathrm{H}), 6.20(\mathrm{~s}, 1 \mathrm{H}), 4.98(\mathrm{~s}, 2 \mathrm{H})$, $2.40-2.30(\mathrm{~m}, 2 \mathrm{H}), 2.13-2.01(\mathrm{~m}, 2 \mathrm{H}), 1.99(\mathrm{~s}, J=4.3 \mathrm{~Hz}, 3 \mathrm{H}), 1.90-1.83(\mathrm{~m}, 2 \mathrm{H})$.

${ }^{13}$ C NMR (100 MHz, CDCl $) \delta 170.93,150.98,148.12,138.30,136.39,134.48,134.40$, $133.78,132.38,132.16,130.59$, 130.26, 128.87, 128.34, 127.95, 127.92, 127.64, 127.44, $126.31,126.22,126.03,125.87,125.64,125.07,121.63,121.46,116.48,115.37,68.06$, $37.47,30.89,23.40,20.66$.

HRMS (ESI-TOF) m/z: [M+H $]^{+}$calcd for $\mathrm{C}_{33} \mathrm{H}_{29} \mathrm{~N}_{2} \mathrm{O}_{2}$ 485.2224; Found 485.2239.<smiles>Cc1ccc2c(c1)C(c1cccs1)=C(CCC(=O)NO)CO2</smiles> 


\section{4-(6-methyl-4-(thiophen-2-yl)-2H-chromen-3-yl)- $N$-(quinolin-8-yl)butanamide (5e)}

$32.2 \mathrm{mg}, 73 \%$ yield, brown sticky liquid.

$\boldsymbol{R}_{f}: 0.50$ (hexane/ethyl acetate, $8: 2 \mathrm{v} / \mathrm{v}$ ).

${ }^{1}$ H NMR (400 MHz, CDCl3) $\delta 9.75(\mathrm{~s}, 1 \mathrm{H}), 8.79(\mathrm{dt}, J=6.5,3.2 \mathrm{~Hz}, 1 \mathrm{H}), 8.75(\mathrm{dd}, J=7.2$, $1.5 \mathrm{~Hz}, 1 \mathrm{H}), 8.15(\mathrm{dd}, J=8.3,1.6 \mathrm{~Hz}, 1 \mathrm{H}), 7.57-7.48(\mathrm{~m}, 2 \mathrm{H}), 7.44(\mathrm{dd}, J=8.3,4.2 \mathrm{~Hz}$, $1 \mathrm{H}), 7.32(\mathrm{dd}, J=5.1,1.1 \mathrm{~Hz}, 1 \mathrm{H}), 7.03(\mathrm{dd}, J=5.1,3.4 \mathrm{~Hz}, 1 \mathrm{H}), 6.94(\mathrm{dd}, J=3.4,1.1 \mathrm{~Hz}$, $1 \mathrm{H}), 6.90(\mathrm{dd}, J=8.1,1.6 \mathrm{~Hz}, 1 \mathrm{H}), 6.76(\mathrm{~d}, J=8.1 \mathrm{~Hz}, 1 \mathrm{H}), 6.61(\mathrm{~d}, J=1.7 \mathrm{~Hz}, 1 \mathrm{H}), 4.81(\mathrm{~s}$, 2H), $2.53(\mathrm{t}, J=7.5 \mathrm{~Hz}, 2 \mathrm{H}), 2.34-2.26(\mathrm{~m}, 2 \mathrm{H}), 2.16(\mathrm{~s}, 3 \mathrm{H}), 1.99-1.92(\mathrm{~m}, 2 \mathrm{H})$.

${ }^{13}$ C NMR (100 MHz, CDCl3) $\delta 170.99,151.03,148.21,138.37,136.97,136.45,134.50$, $134.48,130.65$, 129.06, 127.99, 127.48, 127.13, 126.15, 125.87, 125.16, 125.03, 121.67, $121.53,116.56,115.35,68.23,37.51,31.10,23.83,20.82$.

HRMS (ESI-TOF) m/z: $[\mathrm{M}+\mathrm{H}]^{+}$calcd for $\mathrm{C}_{27} \mathrm{H}_{25} \mathrm{~N}_{2} \mathrm{O}_{2} \mathrm{~S} 441.1631$; Found 441.1653.<smiles>CC(C)(c1ccccc1)c1ccc2c(c1)C(c1ccccc1)=C(CCC(=O)NO)CO2</smiles>

\section{4-(4-phenyl-6-(2-phenylpropan-2-yl)-2H-chromen-3-yl)- $N$-(quinolin-8-yl)butanamide} $\underline{(5 \mathbf{f})}$

$39.3 \mathrm{mg}, 72 \%$ yield, yellow sticky liquid.

$\boldsymbol{R}_{f}: 0.50$ (hexane/ethyl acetate, $8: 2 \mathrm{v} / \mathrm{v}$ ).

${ }^{1}$ H NMR (400 MHz, CDCl $) \delta 9.71(\mathrm{~s}, 1 \mathrm{H}), 8.79$ (dd, $\left.J=4.1,1.5 \mathrm{~Hz}, 1 \mathrm{H}\right), 8.72(\mathrm{dd}, J=7.1$, $1.7 \mathrm{~Hz}, 1 \mathrm{H}), 8.16(\mathrm{dd}, J=8.3,1.5 \mathrm{~Hz}, 1 \mathrm{H}), 7.52(\mathrm{t}, J=4.9 \mathrm{~Hz}, 2 \mathrm{H}), 7.45(\mathrm{dd}, J=8.3,4.2 \mathrm{~Hz}$, 1H), $7.28-7.19(\mathrm{~m}, 6 \mathrm{H}), 7.14-7.12(\mathrm{~m}, 4 \mathrm{H}), 6.88(\mathrm{dd}, J=8.4,2.3 \mathrm{~Hz}, 1 \mathrm{H}), 6.75(\mathrm{~d}, J=8.4$ $\mathrm{Hz}, 1 \mathrm{H}), 6.52$ (d, $J=2.3 \mathrm{~Hz}, 1 \mathrm{H}), 4.84(\mathrm{~s}, 2 \mathrm{H}), 2.47$ (t, $J=7.5 \mathrm{~Hz}, 2 \mathrm{H}), 2.24-2.15(\mathrm{~m}, 2 \mathrm{H})$, $1.95-1.87(\mathrm{~m}, 2 \mathrm{H}), 1.49(\mathrm{~s}, 6 \mathrm{H})$.

${ }^{13}$ C NMR (100 MHz, CDCl3) $\delta 171.10,151.32,150.75,148.23,143.45,138.42,136.74$, $136.50,134.50,132.48,130.53,129.91,129.70,128.42,128.05,127.94,127.54,127.28$, $127.17,126.72,125.52,124.47,124.28,121.71,121.55,116.62,115.01,68.21,42.42,37.58$, $30.72,23.77$.

HRMS (ESI-TOF) m/z: [M+H] $]^{+}$calcd for $\mathrm{C}_{37} \mathrm{H}_{35} \mathrm{~N}_{2} \mathrm{O}_{2}$ 539.2693; Found 539.2685. 
<smiles>O=C(CCC1=C(c2ccccc2)c2cc(-c3ccccc3)ccc2OC1)NO</smiles>

$5 \mathrm{~g}, 81 \%$

4-(4,6-diphenyl-2H-chromen-3-yl)- $N$-(quinolin-8-yl)butanamide (5g)

$40.2 \mathrm{mg}, 81 \%$ yield, yellow sticky liquid.

$\boldsymbol{R}_{f}: 0.50$ (hexane/ethyl acetate, 8:2 v/v).

${ }^{1}$ H NMR (400 MHz, CDCl $) \delta 9.73(\mathrm{~s}, 1 \mathrm{H}), 8.79(\mathrm{~d}, J=3.0 \mathrm{~Hz}, 1 \mathrm{H}), 8.73$ (d, $J=6.9 \mathrm{~Hz}$, $1 \mathrm{H}), 8.16(\mathrm{~d}, J=7.9 \mathrm{~Hz}, 1 \mathrm{H}), 7.53(\mathrm{q}, J=8.0 \mathrm{~Hz}, 2 \mathrm{H}), 7.45(\mathrm{dd}, J=8.1,4.2 \mathrm{~Hz}, 1 \mathrm{H}), 7.39-$ $7.29(\mathrm{~m}, 7 \mathrm{H}), 7.28-7.15(\mathrm{~m}, 4 \mathrm{H}), 6.93(\mathrm{~d}, J=8.2 \mathrm{~Hz}, 1 \mathrm{H}), 6.81(\mathrm{~s}, 1 \mathrm{H}), 4.91(\mathrm{~s}, 2 \mathrm{H}), 2.49$ (t, $J=7.3 \mathrm{~Hz}, 2 \mathrm{H}), 2.29-2.12(\mathrm{~m}, 2 \mathrm{H}), 1.97-1.92(\mathrm{~m}, 2 \mathrm{H})$.

${ }^{13}$ C NMR (100 MHz, CDCl $) \delta$ 171.04, 153.07, 148.23, 141.12, 138.39, 136.68, 136.54, $134.49,134.45,132.20,130.54,129.95,128.70,128.06,127.55,127.51,127.13,126.84$, 126.70, 125.52, 124.71, 121.72, 121.58, 116.64, 115.96, 68.31, 37.56, 30.73, 23.71.

HRMS (ESI-TOF) m/z: [M+H $]^{+}$calcd for $\mathrm{C}_{34} \mathrm{H}_{29} \mathrm{~N}_{3} \mathrm{O}_{2}$ 497.2224; Found 497.2220.<smiles>COc1ccc2c(c1)C(c1ccccc1)=C(CCC(=O)NO)CO2</smiles>

$5 \mathrm{~h}, 86 \%$

\section{4-(6-methoxy-4-phenyl-2H-chromen-3-yl)- $N$-(quinolin-8-yl)butanamide (5h)}

$38.7 \mathrm{mg}, 86 \%$ yield, yellow sticky liquid.

$\boldsymbol{R}_{\boldsymbol{f}}: 0.40$ (hexane/ethyl acetate, $8: 2 \mathrm{v} / \mathrm{v}$ ).

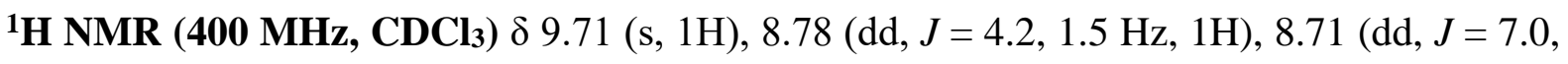
$1.7 \mathrm{~Hz}, 1 \mathrm{H}), 8.15(\mathrm{dd}, J=8.3,1.5 \mathrm{~Hz}, 1 \mathrm{H}), 7.55-7.47$ (m, 2H), $7.44(\mathrm{dd}, J=8.3,4.2 \mathrm{~Hz}$, 1H), $7.36-7.29(\mathrm{~m}, 2 \mathrm{H}), 7.26(\mathrm{dd}, J=8.3,6.2 \mathrm{~Hz}, 1 \mathrm{H}), 7.22-7.12(\mathrm{~m}, 2 \mathrm{H}), 6.80(\mathrm{~d}, J=8.7$ Hz, 1H), 6.63 (dd, $J=8.7,3.0 \mathrm{~Hz}, 1 \mathrm{H}), 6.17$ (d, $J=2.9 \mathrm{~Hz}, 1 \mathrm{H}), 4.80$ (s, 2H), 3.60 (s, 3H), $2.47(\mathrm{t}, J=7.5 \mathrm{~Hz}, 2 \mathrm{H}), 2.25-2.11(\mathrm{~m}, 2 \mathrm{H}), 1.94-1.87(\mathrm{dt}, J=15.1,7.6 \mathrm{~Hz}, 2 \mathrm{H})$.

${ }^{13}$ C NMR (100 MHz, CDCl $) \delta 171.07,154.10,148.23,147.39,138.39,136.69,136.50$, $134.46,132.23,131.25,129.92$, 128.62, 128.03, 127.51, 127.43, 126.26, 121.70, 121.57, $116.62,115.92,112.79,112.15,68.19,55.72,37.55,30.73,23.73$.

HRMS (ESI-TOF) m/z: [M+H] $]^{+}$calcd for $\mathrm{C}_{29} \mathrm{H}_{27} \mathrm{~N}_{2} \mathrm{O}_{3} 451.2016$; Found 451.2025. 
<smiles>Cc1cc(C)c2c(c1)C(c1ccccc1)=C(CCC(=O)NO)CO2</smiles>

4-(6,8-dimethyl-4-phenyl-2H-chromen-3-yl)- $N$-(quinolin-8-yl)butanamide (5i)

$31.0 \mathrm{mg}, 69 \%$ yield, yellow sticky liquid.

$\boldsymbol{R}_{f}: 0.40$ (hexane/ethyl acetate, 8:2 v/v).

${ }^{1}$ H NMR (400 MHz, CDCl $) \delta 9.71(\mathrm{~s}, 1 \mathrm{H}), 8.79(\mathrm{dd}, J=4.2,1.7 \mathrm{~Hz}, 1 \mathrm{H}), 8.72(\mathrm{dd}, J=7.1$, $1.8 \mathrm{~Hz}, 1 \mathrm{H}), 8.16(\mathrm{dd}, J=8.3,1.6 \mathrm{~Hz}, 1 \mathrm{H}), 7.57-7.48(\mathrm{~m}, 2 \mathrm{H}), 7.45(\mathrm{dd}, J=8.3,4.2 \mathrm{~Hz}$, 1H), $7.37-7.24(\mathrm{~m}, 3 \mathrm{H}), 7.21-7.16(\mathrm{~m}, 2 \mathrm{H}), 6.78(\mathrm{~d}, J=0.6 \mathrm{~Hz}, 1 \mathrm{H}), 6.23(\mathrm{~d}, J=1.5 \mathrm{~Hz}$, 1H), $4.82(\mathrm{~s}, 2 \mathrm{H}), 2.47(\mathrm{t}, J=7.5 \mathrm{~Hz}, 2 \mathrm{H}), 2.21(\mathrm{~s}, 3 \mathrm{H}), 2.19-2.13(\mathrm{~m}, 2 \mathrm{H}), 2.09$ (s, 3H), $1.98-1.84(\mathrm{~m}, 2 \mathrm{H})$.

${ }^{13}$ C NMR (100 MHz, CDCl $) \delta 171.13,149.20,148.21,138.39,137.33,136.53,134.52$, $132.57,130.64,130.01,129.71,128.52$, 128.05, 127.55, 127.24, 124.76, 124.66, 124.11, 121.70, 121.53, 116.63, 68.05, 37.60, 30.68, 23.78, 20.75, 15.72.

HRMS (ESI-TOF) m/z: [M+H $]^{+}$calcd for $\mathrm{C}_{30} \mathrm{H}_{29} \mathrm{~N}_{2} \mathrm{O}_{2} 449.2224$; Found 449.2220.<smiles>O=C(CCC1=C(c2ccccc2)c2c(ccc3ccccc23)OC1)NO</smiles>

\section{4-(1-phenyl-3H-benzo[f]chromen-2-yl)- $N$-(quinolin-8-yl)butanamide (5j)}

$39.1 \mathrm{mg}, 83 \%$ yield, brown sticky liquid.

$\boldsymbol{R}_{f}: 0.40$ (hexane/ethyl acetate, 8:2 v/v).

${ }^{1}$ H NMR (400 MHz, CDCl3) $\delta 9.74(\mathrm{~s}, 1 \mathrm{H}), 8.78(\mathrm{dd}, J=4.2,1.4 \mathrm{~Hz}, 1 \mathrm{H}), 8.73$ (d, $J=5.9$ $\mathrm{Hz}, 1 \mathrm{H}), 8.21-8.14(\mathrm{~m}, 2 \mathrm{H}), 7.72-7.68(\mathrm{~m}, 1 \mathrm{H}), 7.54-7.50(\mathrm{~m}, 2 \mathrm{H}), 7.47-7.43(\mathrm{~m}, 3 \mathrm{H})$, $7.41(\mathrm{~d}, J=4.5 \mathrm{~Hz}, 1 \mathrm{H}), 7.38-7.34(\mathrm{~m}, 2 \mathrm{H}), 7.31(\mathrm{~d}, J=7.2 \mathrm{~Hz}, 1 \mathrm{H}), 7.23(\mathrm{~d}, J=8.5 \mathrm{~Hz}$, 2H), $6.77(\mathrm{~d}, J=8.5 \mathrm{~Hz}, 1 \mathrm{H}), 5.03(\mathrm{~s}, 2 \mathrm{H}), 2.51(\mathrm{t}, J=7.4 \mathrm{~Hz}, 2 \mathrm{H}), 2.32-2.21(\mathrm{~m}, 2 \mathrm{H}), 1.98$ $(\mathrm{dt}, J=15.0,7.6 \mathrm{~Hz}, 2 \mathrm{H})$.

${ }^{13}$ C NMR (100 MHz, CDCl $) \delta 171.18,148.86,148.16,137.21,136.62,134.48,133.86$, $132.84,130.59$, 130.06, 128.65, 128.56, 128.29, 128.08, 127.60, 127.42, 126.29, 125.57, 124.38, 123.96, 122.09, 121.69, 121.58, 120.26, 119.74, 116.76, 68.61, 37.60, 30.71, 23.87.

HRMS (ESI-TOF) m/z: [M+H] $]^{+}$calcd for $\mathrm{C}_{32} \mathrm{H}_{27} \mathrm{~N}_{2} \mathrm{O}_{2}$ 471.2067; Found 471.2070. 
<smiles>COc1ccc2[nH]c(=O)c(CCC(=O)NO)c(-c3ccccc3)c2c1</smiles>

4-(6-methoxy-2-oxo-4-phenyl-1,2-dihydroquinolin-3-yl)- $N$-(quinolin-8-yl)butanamide $\underline{(5 \mathbf{k})}$

$33.3 \mathrm{mg}, 72 \%$ yield, brown sticky liquid.

$\boldsymbol{R}_{f}: 0.40$ (hexane/ethyl acetate, $8: 2 \mathrm{v} / \mathrm{v}$ ).

${ }^{1}$ H NMR (400 MHz, CDCl3) $\delta 9.70(\mathrm{~s}, 1 \mathrm{H}), 8.77(\mathrm{dd}, J=4.2,1.7 \mathrm{~Hz}, 1 \mathrm{H}), 8.67(\mathrm{dd}, J=6.7$, $2.2 \mathrm{~Hz}, 1 \mathrm{H}), 8.59(\mathrm{~s}, 1 \mathrm{H}), 8.15(\mathrm{dd}, J=8.3,1.6 \mathrm{~Hz}, 1 \mathrm{H}), 7.51(\mathrm{~d}, J=2.1 \mathrm{~Hz}, 1 \mathrm{H}), 7.50-7.48$ (m, 2H), $7.44(\mathrm{dd}, J=8.3,4.2 \mathrm{~Hz}, 1 \mathrm{H}), 7.39-7.37(\mathrm{~m}, 2 \mathrm{H}), 7.36-7.31(\mathrm{~m}, 2 \mathrm{H}), 7.26$ (s, $1 \mathrm{H}), 6.86(\mathrm{~d}, J=9.0 \mathrm{~Hz}, 2 \mathrm{H}), 3.80(\mathrm{~s}, 3 \mathrm{H}), 2.45(\mathrm{t}, J=7.4 \mathrm{~Hz}, 2 \mathrm{H}), 2.42-2.36(\mathrm{~m}, 2 \mathrm{H}), 1.97$ $-1.86(\mathrm{~m}, 2 \mathrm{H})$.

${ }^{13}$ C NMR (100 MHz, CDCl3) $\delta$ 171.33, 163.74, 156.53, 152.05, 148.20, 138.35, 136.51, $134.50,131.25,130.77,129.99,129.57,129.52,128.04,127.50,121.86,121.69,121.53$, $121.15,116.61,114.26,55.60,37.71,29.42,25.02$.

HRMS (ESI-TOF) m/z: [M+H] $]^{+}$calcd for $\mathrm{C}_{29} \mathrm{H}_{26} \mathrm{~N}_{3} \mathrm{O}_{3} 464.1969$; Found 464.1967.<smiles>COc1cc2c(c(OC)c1OC)c(-c1ccccc1)c(CCC(=O)NO)c(=O)n2C</smiles>

$\mathrm{N}$-(quinolin-8-yl)-4-(5,6,7-trimethoxy-1-methyl-2-oxo-4-phenyl-1,2-dihydroquinolin-3yl)butanamide (5l)

$45.7 \mathrm{mg}, 85 \%$ yield, red sticky liquid.

$\boldsymbol{R}_{f}: 0.150$ (hexane/ethyl acetate, $8: 2 \mathrm{v} / \mathrm{v}$ ).

${ }^{1}$ H NMR (400 MHz, CDCl $) \delta 9.69$ (s, 1H), 8.77 (dd, $\left.J=4.2,1.6 \mathrm{~Hz}, 1 \mathrm{H}\right), 8.67$ (dd, $J=7.0$, $1.8 \mathrm{~Hz}, 1 \mathrm{H}), 8.12(\mathrm{dd}, J=8.3,1.6 \mathrm{~Hz}, 1 \mathrm{H}), 7.50-7.43(\mathrm{~m}, 2 \mathrm{H}), 7.41(\mathrm{dd}, J=8.3,4.3 \mathrm{~Hz}$, 1H), $7.31(\mathrm{t}, J=7.6 \mathrm{~Hz}, 2 \mathrm{H}), 7.19(\mathrm{~m}, 3 \mathrm{H}), 6.56(\mathrm{~s}, 1 \mathrm{H}), 3.96(\mathrm{~s}, 3 \mathrm{H}), 3.75(\mathrm{~s}, 3 \mathrm{H}), 3.73$ (s, $3 \mathrm{H}), 3.15(\mathrm{~s}, 3 \mathrm{H}), 2.48-2.34(\mathrm{~m}, 4 \mathrm{H}), 1.95-1.87(\mathrm{~m}, 2 \mathrm{H})$.

${ }^{13}$ C NMR (125 MHz, CDCl $) \delta 171.83,162.08,155.17,151.54,148.11,145.87,140.54$, $138.41,138.36,136.55,136.33,134.64,129.28,127.94,127.65,127.41,127.21,126.50$, $121.55,121.27,116.57,109.96,92.93,60.87,60.75,56.06,38.52,30.71,28.27,25.46$. 
HRMS (ESI-TOF) m/z: [M+H] $]^{+}$calcd for $\mathrm{C}_{32} \mathrm{H}_{32} \mathrm{~N}_{3} \mathrm{O}_{5}$ 538.2336; Found 538.2346.

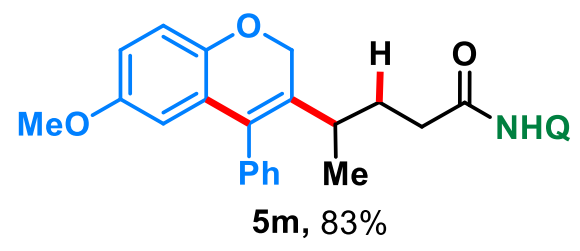

4-(6-methoxy-4-phenyl-2H-chromen-3-yl)- $N$-(quinolin-8-yl)pentanamide (5m)

$40.0 \mathrm{mg}, 83 \%$ yield, yellow sticky liquid.

$\boldsymbol{R}_{f}: 0.50$ (hexane/ethyl acetate, $8: 2 \mathrm{v} / \mathrm{v}$ ).

${ }^{1}$ H NMR (400 MHz, CDCl 3$) \delta 9.72(\mathrm{~s}, 1 \mathrm{H}), 8.77(\mathrm{dd}, J=4.2,1.6 \mathrm{~Hz}, 1 \mathrm{H}), 8.71(\mathrm{dd}, J=7.1$, $1.8 \mathrm{~Hz}, 1 \mathrm{H}), 8.13(\mathrm{dd}, J=8.3,1.6 \mathrm{~Hz}, 1 \mathrm{H}), 7.54-7.47(\mathrm{~m}, 2 \mathrm{H}), 7.44-7.37$ (m, 3H), $7.25(\mathrm{~d}$, $J=8.4 \mathrm{~Hz}, 1 \mathrm{H}), 7.18-7.4(\mathrm{~m}, 2 \mathrm{H}), 6.81(\mathrm{~d}, J=8.7 \mathrm{~Hz}, 1 \mathrm{H}), 6.63(\mathrm{dd}, J=8.7,3.0 \mathrm{~Hz}, 1 \mathrm{H})$, $6.15(\mathrm{~d}, J=3.0 \mathrm{~Hz}, 1 \mathrm{H}), 4.77(\mathrm{~d}, J=2.7 \mathrm{~Hz}, 2 \mathrm{H}), 3.59(\mathrm{~s}, 3 \mathrm{H}), 2.70-2.48(\mathrm{~m}, 2 \mathrm{H}), 2.48-$ $2.33(\mathrm{~m}, 1 \mathrm{H}), 1.91-1.84(\mathrm{~m}, 2 \mathrm{H}), 1.12(\mathrm{~d}, J=7.0 \mathrm{~Hz}, 3 \mathrm{H})$.

${ }^{13}$ C NMR (100 MHz, CDCl $) \delta 171.16,154.03,148.15,147.50,138.27,136.76,136.40$, $134.55,134.40,132.26,129.75,128.56,127.92$, 127.41, 127.31, 126.47, 121.60, 121.48, 116.59, 116.11, 115.86, 114.77, 112.82, 112.28, 64.41, 55.62, 36.18, 34.87, 29.74, 19.12 .

HRMS (ESI-TOF) m/z: [M+H] $]^{+}$calcd for $\mathrm{C}_{30} \mathrm{H}_{29} \mathrm{~N}_{2} \mathrm{O}_{3} 465.2173$; Found 465.2182.<smiles>CCC(CCC(=O)NO)C1=C(c2ccccc2)c2cc(OC)ccc2OC1</smiles>

\section{4-(6-methoxy-4-phenyl-2H-chromen-3-yl)- $N$-(quinolin-8-yl)hexanamide (5n)}

$41.1 \mathrm{mg}, 86 \%$ yield, yellow sticky liquid.

$\boldsymbol{R}_{f}: 0.50$ (hexane/ethyl acetate, $8: 2 \mathrm{v} / \mathrm{v}$ ).

${ }^{1}$ H NMR (400 MHz, CDCl3) $\delta 9.72(\mathrm{~s}, 1 \mathrm{H}), 8.79(\mathrm{dd}, J=4.2,1.7 \mathrm{~Hz}, 1 \mathrm{H}), 8.71(\mathrm{dd}, J=7.1$, $1.8 \mathrm{~Hz}, 1 \mathrm{H}), 8.15(\mathrm{dd}, J=8.3,1.6 \mathrm{~Hz}, 1 \mathrm{H}), 7.56-7.47(\mathrm{~m}, 2 \mathrm{H}), 7.44(\mathrm{dd}, J=8.3,4.2 \mathrm{~Hz}$, 1H), 7.37 (t, $J=7.2 \mathrm{~Hz}, 1 \mathrm{H}), 7.27-7.15(\mathrm{~m}, 4 \mathrm{H}), 6.81(\mathrm{~d}, J=8.7 \mathrm{~Hz}, 1 \mathrm{H}), 6.64(\mathrm{dd}, J=8.7$, $3.0 \mathrm{~Hz}, 1 \mathrm{H}), 6.12(\mathrm{~d}, J=3.0 \mathrm{~Hz}, 1 \mathrm{H}), 4.72(\mathrm{q}, J=13.9 \mathrm{~Hz}, 2 \mathrm{H}), 3.59$ (s, 3H), $2.62-2.55$ (m, $1 \mathrm{H}), 2.47-2.40(\mathrm{~m}, 2 \mathrm{H}), 2.00-1.91(\mathrm{~m}, 1 \mathrm{H}), 1.87-1.77(\mathrm{~m}, 1 \mathrm{H}), 1.54-1.41(\mathrm{~m}, 2 \mathrm{H}), 0.91$ (t, $J=7.4 \mathrm{~Hz}, 3 \mathrm{H})$.

${ }^{13}$ C NMR (100 MHz, CDCl3) $\delta$ 171.27, 154.11, 148.21, 147.65, 138.36, 136.90, 136.45, $134.53,133.83,133.02,130.33,130.10,128.55,128.53,128.00,127.51,127.31,126.68$, 
$121.67,121.47,116.59,115.91,112.92,112.40,64.52,55.72,42.04,36.18,28.66,26.34$, 12.56 .

HRMS (ESI-TOF) m/z: [M+H] $]^{+}$calcd for $\mathrm{C}_{31} \mathrm{H}_{31} \mathrm{~N}_{2} \mathrm{O}_{3} 479.2329$; Found 479.2345.<smiles>COc1ccc2c(c1)C(c1ccccc1)=C(C(CC(=O)NCl)c1ccccc1)CO2</smiles>

5o, $74 \%$

\section{4-(6-methoxy-4-phenyl-2H-chromen-3-yl)-4-phenyl- $N$-(quinolin-8-yl)butanamide (5o)}

$38.9 \mathrm{mg}, 74 \%$ yield, yellow sticky liquid.

$\boldsymbol{R}_{f}: 0.50$ (hexane/ethyl acetate, 8:2 v/v).

${ }^{1}$ H NMR (500 MHz, CDCl $) \delta 9.75(\mathrm{~s}, 1 \mathrm{H}), 8.78(\mathrm{ddd}, J=8.6,5.8,1.4 \mathrm{~Hz}, 2 \mathrm{H}), 8.17(\mathrm{dd}, J$ $=8.3,1.6 \mathrm{~Hz}, 1 \mathrm{H}), 7.60-7.51(\mathrm{~m}, 2 \mathrm{H}), 7.45(\mathrm{~m}, 2 \mathrm{H}), 7.33(\mathrm{~m}, 8 \mathrm{H}), 7.25(\mathrm{~m}, 1 \mathrm{H}), 6.80(\mathrm{dd}, J$ $=8.6,3.2 \mathrm{~Hz}, 1 \mathrm{H}), 6.67(\mathrm{dd}, J=8.7,3.0 \mathrm{~Hz}, 1 \mathrm{H}), 6.21(\mathrm{~d}, J=3.0 \mathrm{~Hz}, 1 \mathrm{H}), 4.86(\mathrm{~d}, J=14.0$ $\mathrm{Hz}, 1 \mathrm{H}), 4.61(\mathrm{~d}, J=14.0 \mathrm{~Hz}, 1 \mathrm{H}), 3.94-3.81(\mathrm{~m}, 1 \mathrm{H}), 3.63(\mathrm{~s}, 3 \mathrm{H}), 2.67-2.53(\mathrm{~m}, 2 \mathrm{H})$, $2.53-2.44(\mathrm{~m}, 1 \mathrm{H}), 2.40-2.26(\mathrm{~m}, 1 \mathrm{H})$.

${ }^{13}$ C NMR (125 MHz, CDCl $) \delta 170.91,154.07,148.17,147.48,141.09,138.33,136.72$, $136.41,134.46,133.11,132.70,130.07,128.74,127.97,127.65,127.60,127.47,126.82$, 126.45, 121.65, 121.52, 116.61, 115.94, 113.19, 112.56, 65.05, 55.69, 45.11, 36.05, 26.97.

HRMS (ESI-TOF) m/z: [M+H] $]^{+}$calcd for $\mathrm{C}_{35} \mathrm{H}_{31} \mathrm{~N}_{2} \mathrm{O}_{3}$ 527.2329; Found 527.2349.<smiles>COc1ccc2c(c1)C(C1=C(CCCC(=O)NO)COc3ccc(OC)cc31)=CCO2</smiles>

\section{4-(6,6'-dimethoxy-2H,2'H-[4,4'-bichromen]-3-yl)-N-(quinolin-8-yl)butanamide (5p)}

$31.5 \mathrm{mg}, 59 \%$ yield, light yellow sticky liquid.

$\boldsymbol{R}_{\boldsymbol{f}}: 0.40$ (hexane/ethyl acetate, $8: 2 \mathrm{v} / \mathrm{v}$ ).

${ }^{1}$ H NMR (500 MHz, CDCl 3$) \delta 9.75(\mathrm{~s}, 1 \mathrm{H}), 8.80(\mathrm{dd}, J=4.2,1.5 \mathrm{~Hz}, 1 \mathrm{H}), 8.75(\mathrm{~d}, J=7.2$ $\mathrm{Hz}, 1 \mathrm{H}), 8.19(\mathrm{~d}, J=8.2 \mathrm{~Hz}, 1 \mathrm{H}), 7.56-7.51(\mathrm{~m}, 2 \mathrm{H}), 7.48(\mathrm{dd}, J=8.2,4.2 \mathrm{~Hz}, 1 \mathrm{H}), 6.81(\mathrm{~d}$, $J=8.7 \mathrm{~Hz}, 1 \mathrm{H}), 6.75(\mathrm{~d}, J=8.7 \mathrm{~Hz}, 1 \mathrm{H}), 6.67-6.64(\mathrm{~m}, 1 \mathrm{H}), 6.62(\mathrm{dd}, J=8.8,3.0 \mathrm{~Hz}, 1 \mathrm{H})$, $6.52(\mathrm{~d}, J=2.9 \mathrm{~Hz}, 1 \mathrm{H}), 6.46(\mathrm{~d}, J=2.9 \mathrm{~Hz}, 1 \mathrm{H}), 5.80(\mathrm{t}, J=3.7 \mathrm{~Hz}, 1 \mathrm{H}), 4.81(\mathrm{~s}, 2 \mathrm{H}), 4.79$ 
(d, $J=3.7 \mathrm{~Hz}, 2 \mathrm{H}), 3.65(\mathrm{~s}, J=5.4 \mathrm{~Hz}, 3 \mathrm{H}), 3.62(\mathrm{~s}, J=6.3 \mathrm{~Hz}, 3 \mathrm{H}), 2.60-2.53(\mathrm{~m}, 2 \mathrm{H})$, $2.37-2.28(\mathrm{~m}, 2 \mathrm{H}), 2.01-1.91(\mathrm{~m}, 2 \mathrm{H})$.

${ }^{13}$ C NMR (125 MHz, CDCl $) \delta 170.99,154.29,154.25,148.27,147.31,136.55,134.47$, $133.40,131.53,128.08,127.97,127.57,124.62$, 123.77, 123.47, 121.76, 121.65, 116.64, 116.49 , 116.27, 116.08, 114.76, 114.09, 113.06, 111.41, 111.15, 67.96, 65.23, 55.79, 55.76, $37.55,30.97,23.76$.

HRMS (ESI-TOF) m/z: [M+H $]^{+}$calcd for $\mathrm{C}_{33} \mathrm{H}_{31} \mathrm{~N}_{2} \mathrm{O}_{5}$ 535.2227; Found 535.2240.

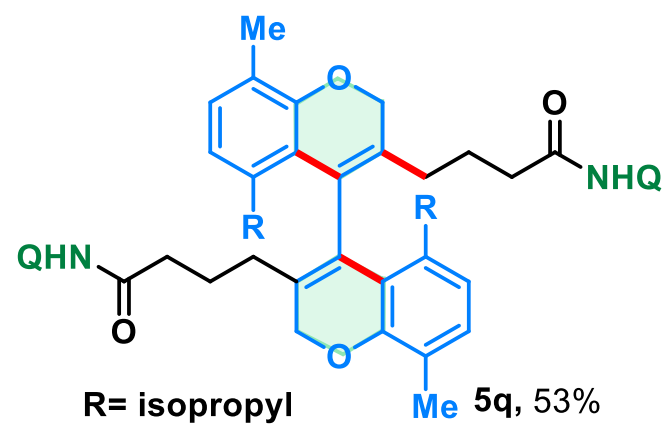

4,4'-(5,5'-diisopropyl-8,8'-dimethyl-2H,2'H-[4,4'-bichromene]-3,3'-divl)bis(N-(quinolin-

\section{8-yl)butanamide)(5q)}

$42.3 \mathrm{mg}, 53 \%$ yield, light yellow liquid.

$\boldsymbol{R}_{f}: 0.30$ (hexane/ethyl acetate, $8: 2 \mathrm{v} / \mathrm{v}$ ).

${ }^{1}$ H NMR (400 MHz, CDCl3) $\delta 9.85$ (s, 1H), 8.79 (t, $\left.J=5.8 \mathrm{~Hz}, 2 \mathrm{H}\right), 8.19$ (d, $J=8.1 \mathrm{~Hz}$, 1H), $7.59-7.50(\mathrm{~m}, 2 \mathrm{H}), 7.47(\mathrm{dd}, J=8.2,4.2 \mathrm{~Hz}, 1 \mathrm{H}), 7.19$ (s, 1H), 7.09 (d, $J=7.6 \mathrm{~Hz}$, 1H), $6.75(\mathrm{~d}, J=7.8 \mathrm{~Hz}, 1 \mathrm{H}), 6.72(\mathrm{~s}, 1 \mathrm{H}), 4.70(\mathrm{~s}, 2 \mathrm{H}), 3.27(\mathrm{dt}, J=13.8,6.9 \mathrm{~Hz}, 1 \mathrm{H}), 2.61$ $(\mathrm{t}, J=7.4 \mathrm{~Hz}, 2 \mathrm{H}), 2.39-2.33(\mathrm{~m}, 2 \mathrm{H}), 2.32(\mathrm{~s}, 3 \mathrm{H}), 2.14(\mathrm{~s}, 3 \mathrm{H}), 2.10-2.01(\mathrm{~m}, 2 \mathrm{H}), 1.18$ (s, 3H), 1.17 (s, 3H).

${ }^{13}$ C NMR (100 MHz, CDCl3) $\delta$ 171.42, 167.67, 155.77, 148.07, 136.35, 134.32, 133.08, $128.14,127.70,126.04$, 121.68, 121.60, 121.46, 120.66, 112.37, 62.96, 37.52, 29.46, 26.67, $23.59,22.91,21.50,20.82$.

HRMS (ESI-TOF) m/z: [M+H] $]^{+}$calcd for $\mathrm{C}_{52} \mathrm{H}_{55} \mathrm{~N}_{4} \mathrm{O}_{4}$ 799.4218; Found 799.4210 .

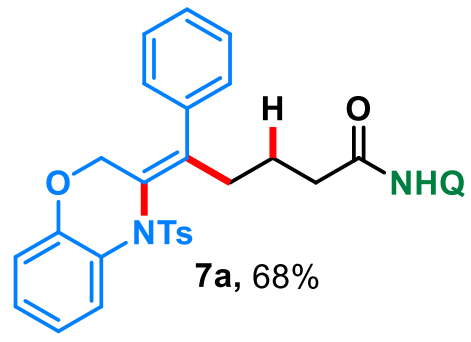




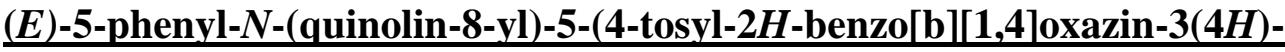

\section{ylidene)pentanamide (7a)}

$40.1 \mathrm{mg}, 68 \%$ yield, yellow sticky liquid.

$\boldsymbol{R}_{f}: 0.40$ (hexane/ethyl acetate, $7: 3 \mathrm{v} / \mathrm{v}$ ).

${ }^{1}$ H NMR (400 MHz, CDCl3) $\delta 9.69(\mathrm{~s}, 1 \mathrm{H}), 8.78(\mathrm{dd}, J=4.2,1.6 \mathrm{~Hz}, 1 \mathrm{H}), 8.70$ (dd, $J=6.6$, $2.2 \mathrm{~Hz}, 1 \mathrm{H}), 8.17(\mathrm{dd}, J=8.3,1.6 \mathrm{~Hz}, 1 \mathrm{H}), 7.69(\mathrm{~d}, J=8.3 \mathrm{~Hz}, 2 \mathrm{H}), 7.57-7.48(\mathrm{~m}, 2 \mathrm{H})$, $7.46(\mathrm{dd}, J=8.3,4.2 \mathrm{~Hz}, 1 \mathrm{H}), 7.35-7.21(\mathrm{~m}, 6 \mathrm{H}), 7.14-7.05(\mathrm{~m}, 2 \mathrm{H}), 6.93(\mathrm{~s}, 1 \mathrm{H}), 6.67(\mathrm{t}$, $J=7.9 \mathrm{~Hz}, 1 \mathrm{H}), 6.29(\mathrm{dd}, J=7.8,1.3 \mathrm{~Hz}, 1 \mathrm{H}), 4.57(\mathrm{~s}, 2 \mathrm{H}), 2.42(\mathrm{t}, J=7.4 \mathrm{~Hz}, 2 \mathrm{H}), 2.37(\mathrm{~s}$, $3 \mathrm{H}), 2.13-2.04(\mathrm{~m}, 2 \mathrm{H}), 1.83(\mathrm{dt}, J=15.0,7.5 \mathrm{~Hz}, 2 \mathrm{H})$.

${ }^{13}$ C NMR (100 MHz, CDCl $) \delta 170.92,148.25,143.80,143.31,138.34,136.59,136.46$, 136.32 , 134.40, 131.92, 130.15, 129.79, 129.60, 128.63, 128.06, 127.58, 127.52, 127.46, 125.32 , 124.61, 122.24, 121.76, 121.69, 121.16, 120.49, 116.62, 68.21, 37.40, 30.50, 23.58, 21.66.

HRMS (ESI-TOF) m/z: [M+H] $]^{+}$calcd for $\mathrm{C}_{35} \mathrm{H}_{32} \mathrm{~N}_{3} \mathrm{O}_{4} \mathrm{~S} 590.2108$; Found 590.2112.

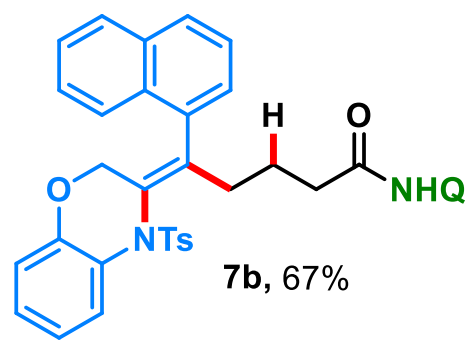

(E)-N,5-di(naphthalen-1-yl)-5-(4-tosyl-2H-benzo[b][1,4]oxazin-3(4H)-

ylidene)pentanamide $(7 \mathbf{b})$

$42.9 \mathrm{mg}, 67 \%$ yield, yellow sticky liquid.

$\boldsymbol{R}_{f}: 0.40$ (hexane/ethyl acetate, $7: 3 \mathrm{v} / \mathrm{v}$ ).

${ }^{1}$ H NMR (500 MHz, CDCl 3$) \delta 9.60(\mathrm{~s}, 1 \mathrm{H}), 8.78(\mathrm{dd}, J=4.2,1.5 \mathrm{~Hz}, 1 \mathrm{H}), 8.68(\mathrm{dd}, J=6.6$, $2.1 \mathrm{~Hz}, 1 \mathrm{H}), 8.18(\mathrm{dd}, J=8.3,1.5 \mathrm{~Hz}, 1 \mathrm{H}), 7.84(\mathrm{t}, J=7.5 \mathrm{~Hz}, 2 \mathrm{H}), 7.74(\mathrm{~d}, J=8.3 \mathrm{~Hz}, 2 \mathrm{H})$, $7.56-7.51(\mathrm{~m}, 3 \mathrm{H}), 7.48-7.42(\mathrm{~m}, 3 \mathrm{H}), 7.40-7.31(\mathrm{~m}, 2 \mathrm{H}), 7.31-7.26(\mathrm{~m}, 3 \mathrm{H}), 6.99$ (s, $1 \mathrm{H}), 6.58(\mathrm{t}, J=7.9 \mathrm{~Hz}, 1 \mathrm{H}), 6.08(\mathrm{dd}, J=7.8,1.1 \mathrm{~Hz}, 1 \mathrm{H}), 4.74(\mathrm{dd}, J=34.6,14.2 \mathrm{~Hz}, 2 \mathrm{H})$, $2.43(\mathrm{~s}, 3 \mathrm{H}), 2.39-2.26(\mathrm{~m}, 2 \mathrm{H}), 2.07-1.92(\mathrm{~m}, 2 \mathrm{H}), 1.82-1.76(\mathrm{~m}, 2 \mathrm{H})$.

${ }^{13}$ C NMR (125 MHz, CDCl $) \delta 170.84,148.21,143.82,143.21,138.33,136.53,136.47$, $134.36,133.82$, 133.77, 132.12, 132.03, 129.99, 129.57, 128.49, 128.25, 128.03, 127.63, $127.54,127.49,126.37,126.14,125.63,125.53,125.20,124.63,122.26,121.75,121.64$, $121.32,120.72,116.54,68.22,37.35,30.73,23.33,21.70$. 
HRMS (ESI-TOF) m/z: [M+H] $]^{+}$calcd for $\mathrm{C}_{39} \mathrm{H}_{34} \mathrm{~N}_{3} \mathrm{O}_{4} \mathrm{~S}$ 640.2265; Found 640.2262.

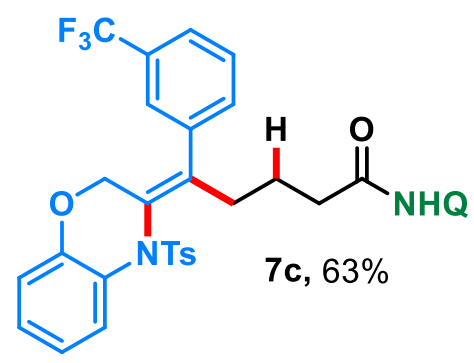

(E)-N-(quinolin-8-yl)-5-(4-tosyl-2H-benzo[b][1,4] oxazin-3(4H)-ylidene)-5-(3(trifluoromethyl)phenyl)pentanamide (7c)

$41.4 \mathrm{mg}, 63 \%$ yield, yellow sticky liquid.

$\boldsymbol{R}_{f}: 0.40$ (hexane/ethyl acetate, $7: 3 \mathrm{v} / \mathrm{v}$ ).

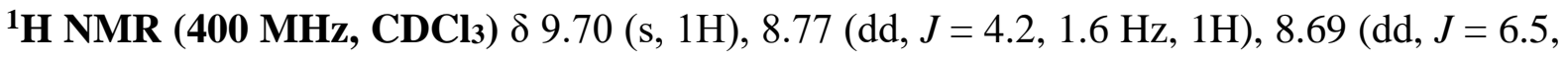
$2.4 \mathrm{~Hz}, 1 \mathrm{H}), 8.18$ (dd, $J=8.3,1.4 \mathrm{~Hz}, 1 \mathrm{H}), 7.69$ (d, $J=8.3 \mathrm{~Hz}, 2 \mathrm{H}), 7.59-7.49$ (m, 3H), $7.49-7.43(\mathrm{~m}, 2 \mathrm{H}), 7.36-7.31(\mathrm{~m}, 3 \mathrm{H}), 7.25(\mathrm{~m}, 2 \mathrm{H}), 6.91(\mathrm{~s}, 1 \mathrm{H}), 6.69(\mathrm{t}, J=8.0 \mathrm{~Hz}, 1 \mathrm{H})$, $6.20(\mathrm{dd}, J=7.8,1.3 \mathrm{~Hz}, 1 \mathrm{H}), 4.58(\mathrm{~s}, 2 \mathrm{H}), 2.43(\mathrm{t}, J=6.8 \mathrm{~Hz}, 2 \mathrm{H}), 2.38$ (s, 3H), $2.15-2.01$ $(\mathrm{m}, 2 \mathrm{H}), 1.90-1.78(\mathrm{~m}, 2 \mathrm{H})$.

${ }^{13}$ C NMR (125 MHz, CDCl3) $\delta 170.69,148.26,143.89,143.27,137.26,136.46,134.34$, $133.36,131.31,130.76,129.65,129.26,128.09,127.55,127.49,126.62$ (q, $J=3.7 \mathrm{~Hz})$, 124.87, 124.72,124.60 (q, $J=3.6 \mathrm{~Hz}), 121.86,121.78$ (d, $J=2.3 \mathrm{~Hz}), 121.41,120.83$, $116.69,77.41,77.16,76.91,68.12,37.28,30.54,23.51,21.68$.

HRMS (ESI-TOF) m/z: [M+H] $]^{+}$calcd for $\mathrm{C}_{36} \mathrm{H}_{31} \mathrm{~F}_{3} \mathrm{~N}_{3} \mathrm{O}_{4} \mathrm{~S}$ 658.1982; Found 658.1980.<smiles>CC1CC(CCC(=O)NO)=C(c2ccccc2)N1[13CH3]</smiles>

4-(5-methyl-2-phenyl-1-tosyl-4,5-dihydro-1H-pyrrol-3-yl)- $N$-(quinolin-8-yl)butanamide

$\underline{(9)}$

$39.4 \mathrm{mg}, 75 \%$ yield, white solid.

$\boldsymbol{R}_{f}: 0.40$ (hexane/ethyl acetate, $5: 5 \mathrm{v} / \mathrm{v}$ ).

${ }^{1}$ H NMR (500 MHz, CDCl $) \delta 9.73(\mathrm{~s}, 1 \mathrm{H}), 8.83(\mathrm{dd}, J=4.2,1.6 \mathrm{~Hz}, 1 \mathrm{H}), 8.76(\mathrm{dd}, J=7.0$, $1.8 \mathrm{~Hz}, 1 \mathrm{H}), 8.20(\mathrm{dd}, J=8.3,1.5 \mathrm{~Hz}, 1 \mathrm{H}), 7.61(\mathrm{~d}, J=8.2 \mathrm{~Hz}, 2 \mathrm{H}), 7.57-7.53(\mathrm{~m}, 2 \mathrm{H})$, $7.49(\mathrm{dd}, J=8.2,4.2 \mathrm{~Hz}, 1 \mathrm{H}), 7.45-7.41(\mathrm{~m}, 2 \mathrm{H}), 7.34-7.31(\mathrm{~m}, 4 \mathrm{H}), 7.29-7.27(\mathrm{~m}, 1 \mathrm{H})$, 
$4.36-4.28(\mathrm{~m}, 1 \mathrm{H}), 2.45-2.39(\mathrm{~m}, 1 \mathrm{H}), 2.38(\mathrm{~s}, 3 \mathrm{H}), 2.37-2.31(\mathrm{~m}, 2 \mathrm{H}), 2.30-2.24(\mathrm{~m}$, $1 \mathrm{H}), 2.23-2.15(\mathrm{~m}, 1 \mathrm{H}), 1.79-1.73(\mathrm{~m}, 3 \mathrm{H}), 1.42(\mathrm{~d}, J=6.6 \mathrm{~Hz}, 3 \mathrm{H})$.

${ }^{13}$ C NMR (125 MHz, CDCl $) \delta 171.01,148.24,143.70,138.32,136.63,135.58,134.77$, $134.41,133.33$, 129.63, 129.46, 128.98, 128.14, 128.09, 127.94, 127.78, 127.53, 121.80, $121.69,116.56,56.85,39.45,37.18,27.61,23.66,22.98,21.62$.

HRMS (ESI-TOF) m/z: [M+H $]^{+}$calcd for $\mathrm{C}_{31} \mathrm{H}_{32} \mathrm{~N}_{3} \mathrm{O}_{3} \mathrm{~S}$ 526.2159; Found 526.2162.

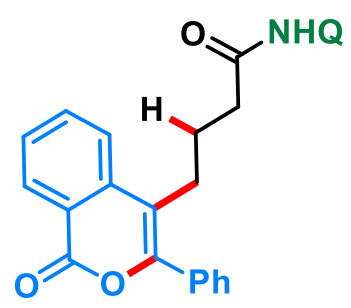

$11,63 \%$

\section{4-(1-oxo-3-phenyl-1H-isochromen-4-yl)- $N$-(quinolin-8-yl)butanamide (11)}

$27.3 \mathrm{mg}, 63 \%$ yield, yellow sticky liquid.

$\boldsymbol{R}_{f}: 0.40$ (hexane/ethyl acetate, $7: 3 \mathrm{v} / \mathrm{v}$ ).

${ }^{1}$ H NMR (500 MHz, CDCl3) $\delta 9.65(\mathrm{~s}, 1 \mathrm{H}), 8.69(\mathrm{dd}, J=4.2,1.6 \mathrm{~Hz}, 1 \mathrm{H}), 8.66(\mathrm{dd}, J=7.3$, $1.4 \mathrm{~Hz}, 1 \mathrm{H}), 8.29(\mathrm{dd}, J=7.9,1.1 \mathrm{~Hz}, 1 \mathrm{H}), 7.80(\mathrm{~d}, J=8.0 \mathrm{~Hz}, 1 \mathrm{H}), 7.77-7.67(\mathrm{~m}, 1 \mathrm{H})$, $7.51-7.39(\mathrm{~m}, 5 \mathrm{H}), 7.36(\mathrm{dd}, J=8.3,4.2 \mathrm{~Hz}, 1 \mathrm{H}), 7.33-7.25(\mathrm{~m}, 3 \mathrm{H}), 2.81-2.70(\mathrm{~m}, 2 \mathrm{H})$, $2.53(\mathrm{t}, J=6.9 \mathrm{~Hz}, 2 \mathrm{H}), 2.11-2.01(\mathrm{~m}, 2 \mathrm{H})$.

${ }^{13}$ C NMR (125 MHz, CDCl $) \delta 170.81,162.52,151.98,148.24,138.32,137.78,136.53$, $135.06,134.38,133.39$, 129.99, 129.49, 129.15, 128.50, 128.06, 128.03, 127.48, 123.89, $121.75,121.66,121.26,116.57,113.51,37.28,26.59,25.45$.

HRMS (ESI-TOF) m/z: [M+H] $]^{+}$calcd for $\mathrm{C}_{28} \mathrm{H}_{23} \mathrm{~N}_{2} \mathrm{O}_{3} 435.1703$; Found 435.1711 .

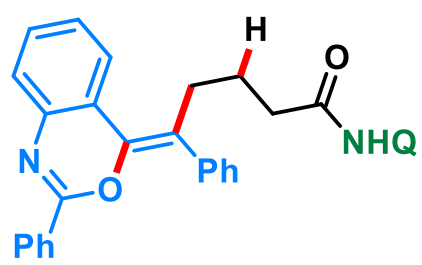

$13,81 \%$

(Z)-5-phenyl-5-(2-phenyl-4H-benzo[d][1,3] oxazin-4-ylidene)- $N$-(quinolin-8yl)pentanamide (13)

$41.3 \mathrm{mg}, 81 \%$ yield, white sticky liquid. 
$\boldsymbol{R}_{f}: 0.40$ (hexane/ethyl acetate, $7: 3 \mathrm{v} / \mathrm{v}$ ).

${ }^{1}$ H NMR (400 MHz, CDCl 3$) \delta 9.79(\mathrm{~s}, 1 \mathrm{H}), 8.81-8.78(\mathrm{~m}, 2 \mathrm{H}), 8.17(\mathrm{~d}, J=7.3 \mathrm{~Hz}, 1 \mathrm{H})$, $7.68(\mathrm{~d}, J=7.8 \mathrm{~Hz}, 1 \mathrm{H}), 7.62(\mathrm{~d}, J=7.6 \mathrm{~Hz}, 2 \mathrm{H}), 7.57-7.50(\mathrm{~m}, 2 \mathrm{H}), 7.47-7.44(\mathrm{~m}, 2 \mathrm{H})$, $7.43-7.33(\mathrm{~m}, 5 \mathrm{H}), 7.33-7.28(\mathrm{~m}, 2 \mathrm{H}), 7.28-7.19(\mathrm{~m}, 3 \mathrm{H}), 2.95-2.81(\mathrm{~m}, 2 \mathrm{H}), 2.63(\mathrm{t}, J$ $=7.2 \mathrm{~Hz}, 2 \mathrm{H}), 2.14-1.99(\mathrm{~m}, 2 \mathrm{H})$.

${ }^{13}$ C NMR (100 MHz, CDCl $) \delta 171.13,156.94,148.05,141.80,140.13,139.46,136.52$, 134.42 , 131.48, 131.08, 129.90, 129.03, 128.35, 128.11, 128.05, 127.97, 127.49, 126.97, $126.69,125.83,125.45,122.50,122.32,121.59,121.47,116.56,37.73,32.21,24.44$.

HRMS (ESI-TOF) m/z: [M+H] $]^{+}$calcd for $\mathrm{C}_{34} \mathrm{H}_{28} \mathrm{~N}_{3} \mathrm{O}_{2}$ 510.2176; Found 510.2180.<smiles>CC(C)(C)CCC(CC(=O)NO)C1=C(c2ccccc2)OC(O)c2ccccc21</smiles>

\section{$\underline{(R)-4-(1-h y d r o x y-3-p h e n y l-1 H}$-isochromen-4-yl)- $N$-(quinolin-8-yl)butanamide (15)}

$26.2 \mathrm{mg}, 60 \%$ yield, brown sticky liquid.

$\boldsymbol{R}_{\boldsymbol{f}}: 0.40$ (hexane/ethyl acetate, $7: 3 \mathrm{v} / \mathrm{v}$ ).

${ }^{1}$ H NMR (400 MHz, CDCl3) $\delta 9.62(\mathrm{~s}, 1 \mathrm{H}), 8.81(\mathrm{dd}, J=4.2,1.7 \mathrm{~Hz}, 1 \mathrm{H}), 8.58(\mathrm{dd}, J=5.8$, $3.2 \mathrm{~Hz}, 1 \mathrm{H}), 8.20(\mathrm{dd}, J=8.3,1.7 \mathrm{~Hz}, 1 \mathrm{H}), 7.52-7.45(\mathrm{~m}, 4 \mathrm{H}), 7.40(\mathrm{td}, J=7.6,1.5 \mathrm{~Hz}$, 1H), 7.33 (dd, $J=7.9,0.9 \mathrm{~Hz}, 3 \mathrm{H}), 7.29-7.17(\mathrm{~m}, 2 \mathrm{H}), 7.15-7.07$ (m, 2H), 6.32 (s, 1H), $5.12(\mathrm{~s}, 1 \mathrm{H}), 2.94(\mathrm{dt}, J=14.6,5.5 \mathrm{~Hz}, 1 \mathrm{H}), 2.90-2.74(\mathrm{~m}, 1 \mathrm{H}), 2.48(\mathrm{ddd}, J=15.2,8.5,5.5$ $\mathrm{Hz}, 1 \mathrm{H}), 2.33$ (ddd, $J=15.4,6.5,5.6 \mathrm{~Hz}, 1 \mathrm{H}), 2.16-1.97$ (m, 2H).

${ }^{13}$ C NMR (100 MHz, CDCl $) \delta 172.14,148.11,147.42,138.21,136.94,135.24,134.33$, $130.15,130.09,129.74,129.28,128.51,128.17,127.95,127.70,126.75,125.70,122.57$, $121.72,121.65,117.11,110.98,92.94,36.81,25.93,23.72$.

HRMS (ESI-TOF) m/z: [M+H] $]^{+}$calcd for $\mathrm{C}_{28} \mathrm{H}_{25} \mathrm{~N}_{2} \mathrm{O}_{3} 437.1860$; Found 437.1853. 


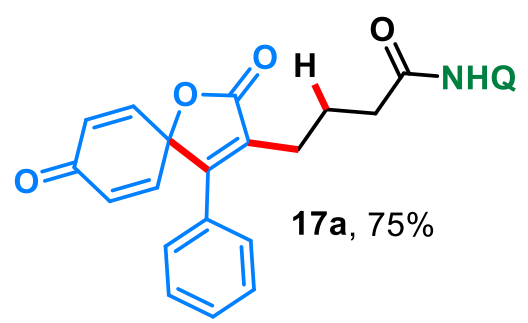

$\underline{\text { 4-(2,8-dioxo-4-phenyl-1-oxaspiro[4.5] deca-3,6,9-trien-3-yl)- } N \text {-(quinolin-8-yl)butanamide }}$ $\underline{(17 \mathbf{a})}$

$33.8 \mathrm{mg}, 75 \%$ yield, brown sticky liquid.

$\boldsymbol{R}_{f}: 0.40$ (hexane/ethyl acetate, $7: 3 \mathrm{v} / \mathrm{v}$ ).

${ }^{1}$ H NMR (400 MHz, CDCl $) \delta 9.74(\mathrm{~s}, 1 \mathrm{H}), 8.80(\mathrm{dd}, J=4.2,1.5 \mathrm{~Hz}, 1 \mathrm{H}), 8.71(\mathrm{dd}, J=6.3$, $2.6 \mathrm{~Hz}, 1 \mathrm{H}), 8.17(\mathrm{dd}, J=8.3,1.5 \mathrm{~Hz}, 1 \mathrm{H}), 7.55-7.49$ (m, 2H), $7.47(\mathrm{dd}, J=8.3,4.2 \mathrm{~Hz}$, 1H), 7.27 (ddd, $J=10.3,7.8,2.1 \mathrm{~Hz}, 3 \mathrm{H}), 7.19-7.07(\mathrm{~m}, 2 \mathrm{H}), 6.64(\mathrm{~d}, J=10.1 \mathrm{~Hz}, 2 \mathrm{H})$, $6.27(\mathrm{~d}, J=10.0 \mathrm{~Hz}, 2 \mathrm{H}), 2.57(\mathrm{t}, J=7.2 \mathrm{~Hz}, 2 \mathrm{H}), 2.55-2.48(\mathrm{~m}, 2 \mathrm{H}), 2.16-2.05(\mathrm{~m}, 2 \mathrm{H})$. ${ }^{13}$ C NMR (100 MHz, CDCl $) \delta 184.04,172.32,170.69,159.62,148.26,143.29,138.30$, 136.62 , 134.42, 131.74, 131.51, 130.13, 129.65, 128.92, 128.08, 127.78, 127.49, 121.81, $121.71,116.60,82.14,37.29,24.68,23.43$.

HRMS (ESI-TOF) m/z: [M+H] $]^{+}$calcd for $\mathrm{C}_{28} \mathrm{H}_{23} \mathrm{~N}_{2} \mathrm{O}_{4} 451.1652$; Found 451.1650 .

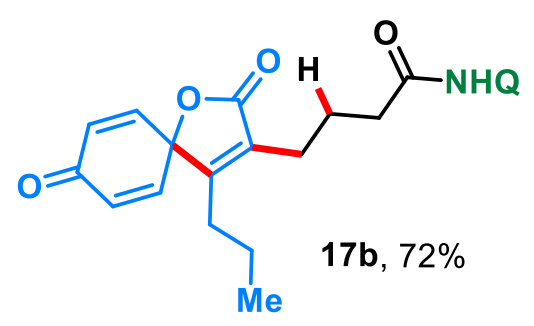

4-(2,8-dioxo-4-propyl-1-oxaspiro[4.5] deca-3,6,9-trien-3-yl)- $N$-(quinolin-8-yl)butanamide $\underline{(17 b)}$

$30.0 \mathrm{mg}, 72 \%$ yield, brown sticky liquid.

$\boldsymbol{R}_{f}: 0.40$ (hexane/ethyl acetate, $7: 3 \mathrm{v} / \mathrm{v}$ ).

${ }^{1}$ H NMR (500 MHz, CDCl $) \delta 9.85(\mathrm{~s}, 1 \mathrm{H}), 8.83(\mathrm{dd}, J=4.2,1.5 \mathrm{~Hz}, 1 \mathrm{H}), 8.77(\mathrm{dd}, J=7.0$, $1.5 \mathrm{~Hz}, 1 \mathrm{H}), 8.20(\mathrm{dd}, J=8.3,1.4 \mathrm{~Hz}, 1 \mathrm{H}), 7.59-7.51(\mathrm{~m}, 2 \mathrm{H}), 7.49$ (dd, $J=8.3,4.2 \mathrm{~Hz}$, $1 \mathrm{H}), 6.50(\mathrm{~d}, J=10.1 \mathrm{~Hz}, 2 \mathrm{H}), 6.39(\mathrm{~d}, J=10.1 \mathrm{~Hz}, 2 \mathrm{H}), 2.68(\mathrm{t}, J=7.1 \mathrm{~Hz}, 2 \mathrm{H}), 2.59-2.45$ (m, 2H), $2.18-2.07$ (m, 4H), $1.51-1.44(\mathrm{~m}, 2 \mathrm{H}), 0.89$ (t, $J=7.3 \mathrm{~Hz}, 3 \mathrm{H})$.

${ }^{13}$ C NMR (125 MHz, CDCl3) $\delta$ 184.37, 172.81, 170.81, 161.54, 148.28, 143.96, 138.28, $136.66,134.40,131.48,130.48,128.09,127.51,121.81$, 121.76, 116.63, 82.31, 37.29, 27.92, $24.23,23.55,22.65,14.45$. 
HRMS (ESI-TOF) m/z: [M+H $]^{+}$calcd for $\mathrm{C}_{25} \mathrm{H}_{25} \mathrm{~N}_{2} \mathrm{O}_{4} 417.1809$; Found 417.1815.

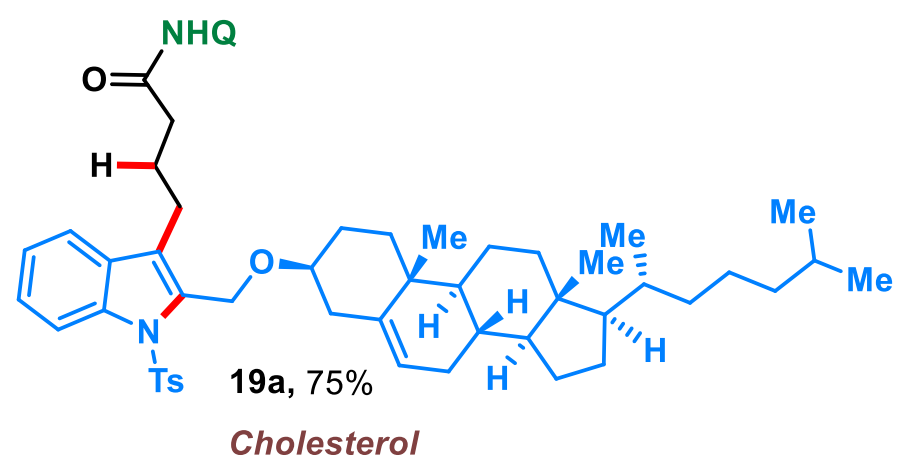

4-(2-((((3S,8S,9S,10R,13R,14S,17R)-10,13-dimethyl-17-((R)-6-methylheptan-2-yl)-

$\underline{2,3,4,7,8,9,10,11,12,13,14,15,16,17-t e t r a d e c a h y d r o-1 H}$-cyclopenta[a]phenanthren-3-

yl)oxy)methyl)-1-tosyl-1H-indol-3-yl)- $N$-(quinolin-8-yl)butanamide (19a)

$66.2 \mathrm{mg}, 75 \%$ yield, brown solid.

$\boldsymbol{R}_{f}: 0.40$ (hexane/ethyl acetate, $6: 4 \mathrm{v} / \mathrm{v}$ ).

${ }^{1}$ H NMR (500 MHz, CDCl3) $\delta 9.78(\mathrm{~s}, 1 \mathrm{H}), 8.82(\mathrm{dd}, J=4.3,1.6 \mathrm{~Hz}, 2 \mathrm{H}), 8.19(\mathrm{dd}, J=8.3$, $1.6 \mathrm{~Hz}, 1 \mathrm{H}), 8.13(\mathrm{~d}, J=8.4 \mathrm{~Hz}, 1 \mathrm{H}), 7.92(\mathrm{~d}, J=8.4 \mathrm{~Hz}, 2 \mathrm{H}), 7.60-7.51$ (m, 3H), 7.49 (dd, $J=8.3,4.2 \mathrm{~Hz}, 1 \mathrm{H}), 7.33-7.28(\mathrm{~m}, 1 \mathrm{H}), 7.23(\mathrm{t}, J=7.5 \mathrm{~Hz}, 1 \mathrm{H}), 7.18(\mathrm{~d}, J=8.2 \mathrm{~Hz}, 2 \mathrm{H})$, $5.31-5.23(\mathrm{~m}, 1 \mathrm{H}), 4.93(\mathrm{~s}, 2 \mathrm{H}), 3.39-3.33(\mathrm{~m}, 1 \mathrm{H}), 2.92(\mathrm{t}, J=7.4 \mathrm{~Hz}, 2 \mathrm{H}), 2.57(\mathrm{t}, J=$ $7.2 \mathrm{~Hz}, 2 \mathrm{H}), 2.43-2.33(\mathrm{~m}, 1 \mathrm{H}), 2.28(\mathrm{~s}, 3 \mathrm{H}), 2.19-2.12(\mathrm{~m}, 2 \mathrm{H}), 2.02(\mathrm{~d}, J=12.7 \mathrm{~Hz}$, 1H), $1.97-1.90(\mathrm{~m}, 2 \mathrm{H}), 1.88-1.81(\mathrm{~m}, 1 \mathrm{H}), 1.77(\mathrm{dd}, J=10.0,3.4 \mathrm{~Hz}, 1 \mathrm{H}), 1.58-1.51$ $(\mathrm{m}, 3 \mathrm{H}), 1.48-1.35(\mathrm{~m}, 7 \mathrm{H}), 1.28(\mathrm{~d}, J=6.9 \mathrm{~Hz}, 2 \mathrm{H}), 1.20-1.07(\mathrm{~m}, 6 \mathrm{H}), 1.04-0.98(\mathrm{~m}$, $2 \mathrm{H}), 0.96-0.92(\mathrm{~m}, 6 \mathrm{H}), 0.90(\mathrm{dd}, J=6.6,2.3 \mathrm{~Hz}, 6 \mathrm{H}), 0.69$ (s, 3H), 0.10 (s, 2H).

${ }^{13}$ C NMR (125 MHz, CDCl3) $\delta$ 171.33, 148.29, 144.45, 140.90, 138.44, 136.71, 136.51, $136.25,134.61,133.87,129.90,129.50,128.12$, 127.60, 127.24, 125.17, 123.91, 123.40, $121.76,121.57,119.57,116.61,115.15,79.24,59.55,56.88,56.29,50.26,42.45,39.92$, $39.67,39.05,37.31,37.03,36.93,36.34,35.93,32.05,32.00,28.38,28.31,28.17,25.51$, 24.42, 23.98, 23.66, 22.97, 22.71, 21.61, 21.18, 19.46, 18.87, 12.00, 1.16.

HRMS (ESI-TOF) m/z: [M+H] $]^{+}$calcd for $\mathrm{C}_{56} \mathrm{H}_{72} \mathrm{~N}_{3} \mathrm{O}_{4} \mathrm{~S} 882.5238$; Found 882.5218. 


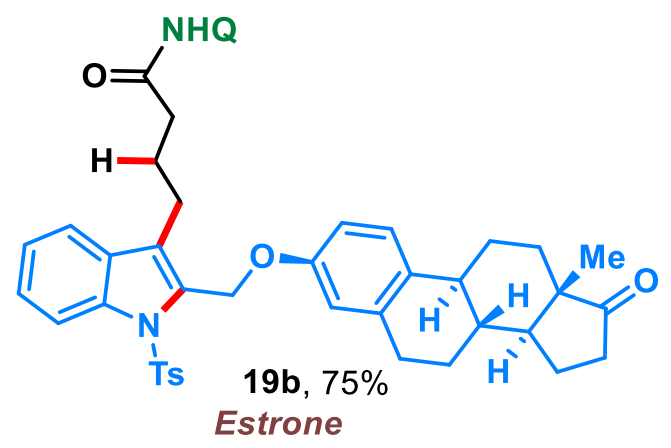

4-(2-((( (8R,9S,13S,14S)-13-methyl-17-oxo-7,8,9,11,12,13,14,15,16,17-decahydro-6Hcyclopenta[a]phenanthren-3-yl)oxy)methyl)-1-tosyl-1H-indol-3-yl)- $N$-(quinolin-8yl)butanamide (19b)

$57.5 \mathrm{mg}, 75 \%$ yield, brown sticky liquid.

$\boldsymbol{R}_{f}: 0.40$ (hexane/ethyl acetate, $6: 4 \mathrm{v} / \mathrm{v}$ ).

${ }^{1}$ H NMR (500 MHz, CDCl $) \delta 9.73(\mathrm{~s}, 1 \mathrm{H}), 8.81(\mathrm{dd}, J=4.1,1.3 \mathrm{~Hz}, 1 \mathrm{H}), 8.80-8.72(\mathrm{~m}$, $1 \mathrm{H}), 8.23(\mathrm{~d}, J=8.4 \mathrm{~Hz}, 1 \mathrm{H}), 8.19(\mathrm{dd}, J=8.2,1.2 \mathrm{~Hz}, 1 \mathrm{H}), 7.88(\mathrm{~d}, J=8.3 \mathrm{~Hz}, 2 \mathrm{H}), 7.62(\mathrm{~d}$, $J=7.8 \mathrm{~Hz}, 1 \mathrm{H}), 7.59-7.51(\mathrm{~m}, 2 \mathrm{H}), 7.48(\mathrm{dd}, J=8.2,4.2 \mathrm{~Hz}, 1 \mathrm{H}), 7.42-7.35(\mathrm{~m}, 1 \mathrm{H})$, $7.28(\mathrm{~d}, J=7.6 \mathrm{~Hz}, 1 \mathrm{H}), 7.20(\mathrm{~d}, J=8.2 \mathrm{~Hz}, 2 \mathrm{H}), 7.14(\mathrm{~d}, J=8.5 \mathrm{~Hz}, 1 \mathrm{H}), 6.73(\mathrm{dd}, J=8.5$, $2.3 \mathrm{~Hz}, 1 \mathrm{H}), 6.67(\mathrm{~s}, 1 \mathrm{H}), 5.41(\mathrm{~s}, 2 \mathrm{H}), 2.91(\mathrm{t}, J=7.3 \mathrm{~Hz}, 2 \mathrm{H}), 2.89-2.78(\mathrm{~m}, 2 \mathrm{H}), 2.58-$ $2.49(\mathrm{~m}, 3 \mathrm{H}), 2.35(\mathrm{t}, J=9.8 \mathrm{~Hz}, 2 \mathrm{H}), 2.31(\mathrm{~s}, 3 \mathrm{H}), 2.24-2.12(\mathrm{~m}, 4 \mathrm{H}), 2.02-1.96(\mathrm{~m}, 2 \mathrm{H})$, $1.72-1.57(\mathrm{~m}, 3 \mathrm{H}), 1.55-1.48(\mathrm{~m}, 3 \mathrm{H}), 0.93(\mathrm{~s}, 3 \mathrm{H})$.

${ }^{13}$ C NMR (125 MHz, CDCl3) $\delta$ 221.19, 171.17, 156.35, 148.23, 144.65, 138.34, 137.84, $136.78,136.47,135.97,134.48,132.55,131.60,129.67,129.53,128.03,127.51,127.24$, $126.44,125.61,125.07,123.57,121.73,121.52$, 119.82, 116.56, 115.13, 114.61, 112.71, $59.97,50.49,48.11,44.05,38.36,36.64,35.98,31.67,29.72,26.61,25.95,25.28,23.51$, $21.68,21.62,13.95$.

HRMS (ESI-TOF) m/z: [M+H] $]^{+}$calcd for $\mathrm{C}_{47} \mathrm{H}_{48} \mathrm{~N}_{3} \mathrm{O}_{5} \mathrm{~S} 766.3309$; Found 766.3314.

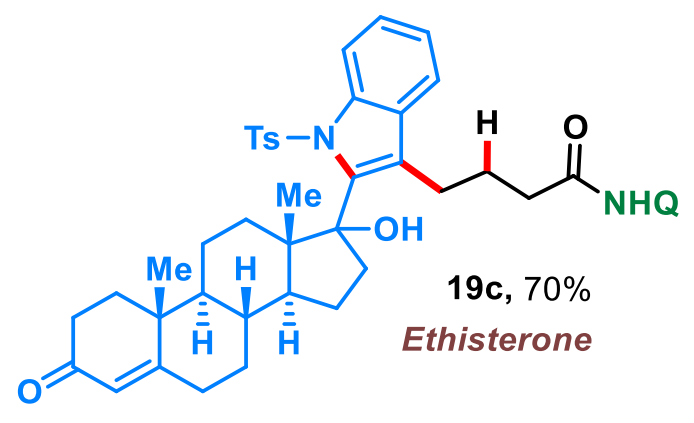


4-(2-((8R,9S,10R,13S,14S)-17-hydroxy-10,13-dimethyl-3-oxo-

$\underline{2,3,6,7,8,9,10,11,12,13,14,15,16,17-t e t r a d e c a h y d r o-1 H}$-cyclopenta[a]phenanthren-17-yl)-

\section{1-tosyl-1H-indol-3-yl)- $N$-(quinolin-8-yl)butanamide (19c)}

$53.9 \mathrm{mg}, 70 \%$ yield, yellow sticky liquid

$\boldsymbol{R}_{f}: 0.40$ (hexane/ethyl acetate, $6: 4 \mathrm{v} / \mathrm{v}$ ).

${ }^{1}$ H NMR (500 MHz, CDCl $) \delta 9.90(\mathrm{~s}, 1 \mathrm{H}), 8.85(\mathrm{~d}, J=7.2 \mathrm{~Hz}, 1 \mathrm{H}), 8.81(\mathrm{dd}, J=4.0,1.2$ $\mathrm{Hz}, 1 \mathrm{H}), 8.18(\mathrm{dd}, J=8.2,1.1 \mathrm{~Hz}, 1 \mathrm{H}), 8.01-7.90(\mathrm{~m}, 1 \mathrm{H}), 7.76(\mathrm{~d}, J=8.3 \mathrm{~Hz}, 2 \mathrm{H}), 7.71-$ $7.62(\mathrm{~m}, 1 \mathrm{H}), 7.61-7.50(\mathrm{~m}, 2 \mathrm{H}), 7.47(\mathrm{dd}, J=8.2,4.2 \mathrm{~Hz}, 1 \mathrm{H}), 7.27-7.17(\mathrm{~m}, 4 \mathrm{H}), 6.11$ (s, 1H), $5.58(\mathrm{~s}, 1 \mathrm{H}), 2.97-2.91(\mathrm{~m}, 1 \mathrm{H}), 2.90-2.80(\mathrm{~m}, 2 \mathrm{H}), 2.77(\mathrm{dd}, J=11.1,6.6 \mathrm{~Hz}$, 2H), $2.43(\mathrm{~d}, J=12.8 \mathrm{~Hz}, 1 \mathrm{H}), 2.32(\mathrm{~s}, 4 \mathrm{H}), 2.26(\mathrm{t}, J=11.6 \mathrm{~Hz}, 3 \mathrm{H}), 1.97$ (d, $J=13.6 \mathrm{~Hz}$, $1 \mathrm{H}), 1.88(\mathrm{dd}, J=9.4,3.6 \mathrm{~Hz}, 1 \mathrm{H}), 1.82-1.72(\mathrm{~m}, 1 \mathrm{H}), 1.58-1.56(\mathrm{~m}, 2 \mathrm{H}), 1.52$ - $1.44(\mathrm{~m}$, $3 \mathrm{H}), 1.38$ - $1.35(\mathrm{~m}, 1 \mathrm{H}), 1.28-1.27(\mathrm{~m}, 1 \mathrm{H}), 1.24-1.18(\mathrm{~m}, 1 \mathrm{H}), 1.11(\mathrm{~s}, 3 \mathrm{H}), 1.08(\mathrm{~s}, 3 \mathrm{H})$, $0.91-0.84(\mathrm{~m}, 2 \mathrm{H}), 0.75-0.68(\mathrm{~m}, 1 \mathrm{H}), 0.65-0.57(\mathrm{~m}, 1 \mathrm{H})$.

${ }^{13}$ C NMR (125 MHz, CDCl3) $\delta$ 199.56, 171.15, 170.87, 148.25, 144.68, 142.28, 138.29, $136.84,136.76,136.49,134.40,130.20,129.86,127.97,127.48,126.42,125.04,123.70$, $123.43,121.98,121.71,121.62,119.50,116.55,115.66,85.61,52.76,51.67,49.78,38.99$, $38.38,38.12,36.36,35.34,34.76,33.88,32.68,31.64,27.03,25.12$, 24.35, 21.57, 21.15, $17.29,14.55$.

HRMS (ESI-TOF) m/z: [M+H] $]^{+}$calcd for $\mathrm{C}_{47} \mathrm{H}_{52} \mathrm{~N}_{3} \mathrm{O}_{5} \mathrm{~S} 770.3622$; Found 770.3649 .

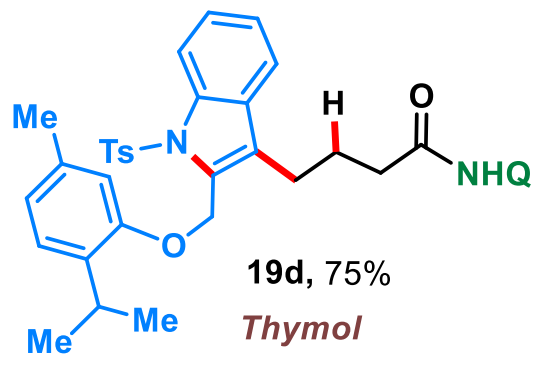

\section{4-(2-((2-isopropyl-5-methylphenoxy)methyl)-1-tosyl-1H-indol-3-yl)- $N$-(quinolin-8-}

yl)butanamide (19d)

$48.4 \mathrm{mg}, 75 \%$ yield, white solid.

$\boldsymbol{R}_{f}: 0.40$ (hexane/ethyl acetate, $6: 4 \mathrm{v} / \mathrm{v}$ ).

${ }^{1}$ H NMR (400 MHz, CDCl3) $\delta 9.73(\mathrm{~s}, 1 \mathrm{H}), 8.83$ - 8.70 (m, 2H), $8.24-8.10$ (m, 2H), 7.85 $7.74(\mathrm{~m}, 2 \mathrm{H}), 7.68-7.61(\mathrm{~m}, 1 \mathrm{H}), 7.57-7.48(\mathrm{~m}, 2 \mathrm{H}), 7.45(\mathrm{dd}, J=8.3,4.2 \mathrm{~Hz}, 1 \mathrm{H}), 7.35$ $(\mathrm{ddd}, J=8.5,7.3,1.3 \mathrm{~Hz}, 1 \mathrm{H}), 7.30-7.21(\mathrm{~m}, 1 \mathrm{H}), 7.13(\mathrm{~d}, J=8.0 \mathrm{~Hz}, 2 \mathrm{H}), 7.09$ (d, $J=7.7$ $\mathrm{Hz}, 1 \mathrm{H}), 6.88(\mathrm{~s}, 1 \mathrm{H}), 6.79(\mathrm{~d}, J=7.7 \mathrm{~Hz}, 1 \mathrm{H}), 5.45$ (s, 2H), $3.12(\mathrm{dt}, J=13.8,6.9 \mathrm{~Hz}, 1 \mathrm{H})$, 
$2.97-2.83(\mathrm{~m}, 2 \mathrm{H}), 2.54(\mathrm{t}, J=7.2 \mathrm{~Hz}, 2 \mathrm{H}), 2.33(\mathrm{~s}, 3 \mathrm{H}), 2.28(\mathrm{~s}, 3 \mathrm{H}), 2.21-2.09(\mathrm{~m}, 2 \mathrm{H})$, $1.11(\mathrm{~s}, 3 \mathrm{H}), 1.09(\mathrm{~s}, 3 \mathrm{H})$.

${ }^{13}$ C NMR (100 MHz, CDCl $) \delta 171.01,155.50,148.24,144.60,138.35,136.82,136.52$, 136.47 , 135.95, 134.47, 131.96, 129.80, 129.58, 128.03, 127.51, 126.93, 125.90, 125.57, $125.47,123.62,122.02,121.73,121.58,119.91,116.56,115.22,113.45,60.54,37.00,25.96$, $25.61,23.64,23.23,21.62,21.46$.

HRMS (ESI-TOF) m/z: [M+H] $]^{+}$calcd for $\mathrm{C}_{39} \mathrm{H}_{40} \mathrm{~N}_{3} \mathrm{O}_{4} \mathrm{~S}$ 646.2734; Found 646.2742.<smiles>Cc1ccc(C(C)C)c2c1C(c1ccccc1)=C(CC(CC(=O)NO)c1ccccc1)CO2</smiles>

4-(8-isopropyl-5-methyl-4-phenyl-2H-chromen-3-yl)-N-(quinolin-8-yl)butanamide (19e) $33.4 \mathrm{mg}, 70 \%$ yield, brown sticky liquid.

$\boldsymbol{R}_{f}: 0.40$ (hexane/ethyl acetate, 8:2 v/v).

${ }^{1}$ H NMR (400 MHz, CDCl3) $\delta 9.72(\mathrm{~s}, 1 \mathrm{H}), 8.79(\mathrm{dd}, J=4.2,1.6 \mathrm{~Hz}, 1 \mathrm{H}), 8.75$ (dd, $J=7.3$, $1.5 \mathrm{~Hz}, 1 \mathrm{H}), 8.16(\mathrm{dd}, J=8.3,1.6 \mathrm{~Hz}, 1 \mathrm{H}), 7.57-7.49$ (m, 2H), 7.45 (dd, $J=8.3,4.2 \mathrm{~Hz}$, 1H), $7.29-7.15(\mathrm{~m}, 5 \mathrm{H}), 7.00(\mathrm{~d}, J=7.8 \mathrm{~Hz}, 1 \mathrm{H}), 6.63$ (d, $J=7.8 \mathrm{~Hz}, 1 \mathrm{H}), 4.59$ (s, 2H), $3.31(\mathrm{dt}, J=13.8,6.9 \mathrm{~Hz}, 1 \mathrm{H}), 2.51(\mathrm{t}, J=7.5 \mathrm{~Hz}, 2 \mathrm{H}), 2.41-2.30(\mathrm{~m}, 2 \mathrm{H}), 2.02-1.90(\mathrm{~m}$, 2H), $1.54(\mathrm{~s}, 3 \mathrm{H}), 1.26(\mathrm{~d}, J=6.9 \mathrm{~Hz}, 6 \mathrm{H})$.

${ }^{13}$ C NMR (100 MHz, CDCl3) $\delta$ 171.18, 152.66, 151.21, 148.21, 139.97, 138.42, 136.48, $134.55,133.61,133.22$, 133.08, 131.97, 129.88, 128.26, 128.04, 127.54, 126.80, 125.03, 124.79, 124.73, 121.70, 121.52, 116.58, 67.86, 37.65, 30.92, 27.04, 24.25, 22.91, 22.14.

HRMS (ESI-TOF) m/z: [M+H] $]^{+}$calcd for $\mathrm{C}_{32} \mathrm{H}_{33} \mathrm{~N}_{2} \mathrm{O}_{2}$ 477.2537; Found 477.2538.

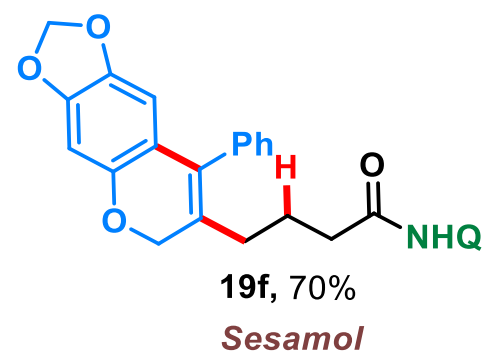

$\underline{\text { 4-(8-phenyl-6H-[1,3]dioxolo[4,5-g]chromen-7-yl)- } N \text {-(quinolin-8-yl)butanamide (19f) }}$ $32.5 \mathrm{mg}, 70 \%$ yield, black sticky liquid. 
$\boldsymbol{R}_{f}: 0.40$ (hexane/ethyl acetate, $8: 2 \mathrm{v} / \mathrm{v}$ ).

${ }^{1}$ H NMR (500 MHz, CDCl $) \delta 9.75(\mathrm{~s}, 1 \mathrm{H}), 8.82(\mathrm{dd}, J=4.2,1.5 \mathrm{~Hz}, 1 \mathrm{H}), 8.74(\mathrm{dd}, J=7.2$, $1.3 \mathrm{~Hz}, 1 \mathrm{H}), 8.20(\mathrm{~d}, J=8.0 \mathrm{~Hz}, 1 \mathrm{H}), 7.58-7.52(\mathrm{~m}, 2 \mathrm{H}), 7.49(\mathrm{dd}, J=8.2,4.2 \mathrm{~Hz}, 1 \mathrm{H})$, $7.35(\mathrm{t}, J=7.3 \mathrm{~Hz}, 2 \mathrm{H}), 7.29(\mathrm{~d}, J=6.1 \mathrm{~Hz}, 1 \mathrm{H}), 7.21-7.17(\mathrm{~m}, 2 \mathrm{H}), 6.47(\mathrm{~s}, 1 \mathrm{H}), 6.10$ (s, 1H), $5.86(\mathrm{~s}, 2 \mathrm{H}), 4.78(\mathrm{~s}, 2 \mathrm{H}), 2.49(\mathrm{t}, J=7.5 \mathrm{~Hz}, 2 \mathrm{H}), 2.26-2.10(\mathrm{~m}, 2 \mathrm{H}), 1.99-1.77$ (m, 2H)

${ }^{13}$ C NMR (125 MHz, CDCl3) $\delta$ 171.18, 148.82, 148.17, 148.15, 146.93, 141.87, 138.26, $137.10,134.45,132.32$, 131.17, 129.91, 128.65, 128.08, 127.62, 127.42, 121.71, 121.59, $118.72,105.79,101.11,98.27,68.37,37.58,30.61,23.87$.

HRMS (ESI-TOF) m/z: $[\mathrm{M}+\mathrm{H}]^{+}$calcd for $\mathrm{C}_{29} \mathrm{H}_{25} \mathrm{~N}_{2} \mathrm{O}_{4} 465.1809$; Found 465.1805 .

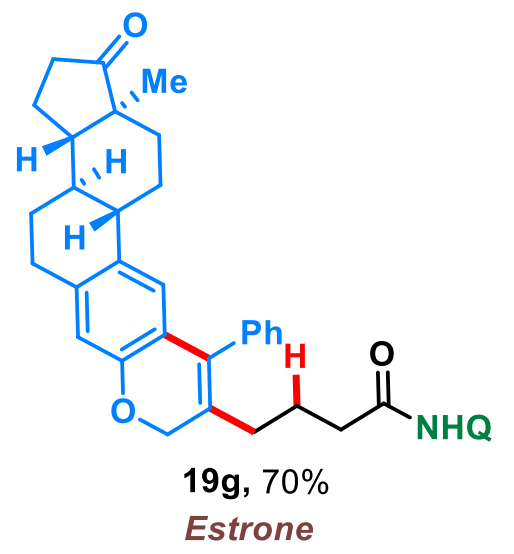

4-((3aS,3bR,11bS,13aS)-13a-methyl-1-oxo-10-phenyl-1,2,3,3a,3b,4,5,8,11b,12,13,13adodecahydrocyclopenta[5,6]naphtho[1,2-g]chromen-9-yl)-N-(quinolin-8-yl)butanamide $\underline{(19 g)}$

$41.7 \mathrm{mg}, 70 \%$ yield, brown sticky liquid.

$\boldsymbol{R}_{\boldsymbol{f}}: 0.40$ (hexane/ethyl acetate, $8: 2 \mathrm{v} / \mathrm{v}$ ).

${ }^{1}$ H NMR (400 MHz, CDCl 3$) \delta 9.70(\mathrm{~s}, 1 \mathrm{H}), 8.79(\mathrm{dd}, J=2.7,1.5 \mathrm{~Hz}, 1 \mathrm{H}), 8.77-8.66(\mathrm{~m}$, 1H), $8.23-8.06(\mathrm{~m}, 1 \mathrm{H}), 7.57-7.48(\mathrm{~m}, 2 \mathrm{H}), 7.45(\mathrm{dd}, J=7.4,4.2 \mathrm{~Hz}, 1 \mathrm{H}), 7.35$ (t, $J=7.2$ $\mathrm{Hz}, 1 \mathrm{H}), 7.31-7.17(\mathrm{~m}, 4 \mathrm{H}), 7.15-7.04(\mathrm{~m}, 1 \mathrm{H}), 6.55$ (d, J = 42.2 Hz, 1H), $4.81(\mathrm{~s}, 1 \mathrm{H})$, $4.54(\mathrm{dd}, J=38.5,12.8 \mathrm{~Hz}, 1 \mathrm{H}), 2.94-2.75$ (m, 1H), $2.58-2.39$ (m, 3H), $2.36-2.29$ (m, 1H), $2.18-2.05(\mathrm{~m}, 3 \mathrm{H}), 1.98-1.85(\mathrm{~m}, 4 \mathrm{H}), 1.78(\mathrm{dd}, J=13.7,6.4 \mathrm{~Hz}, 1 \mathrm{H}), 1.61-1.29$ $(\mathrm{m}, 7 \mathrm{H}), 1.12-1.02(\mathrm{~m}, 1 \mathrm{H}), 0.85(\mathrm{~d}, J=10.9 \mathrm{~Hz}, 3 \mathrm{H})$

${ }^{13}$ C NMR (100 MHz, CDCl3) $\delta$ 221.03, 220.99, 171.03, 153.82, 151.27, 148.12, 140.19, $138.30,136.92$, 136.78, 136.41, 134.92, 134.41, 133.95, 132.69, 132.60, 132.35, 132.17, $129.77,129.34,128.49,127.94,127.92,127.43,127.29,126.63,125.55,124.59,122.94$, 
$122.78,121.60,121.44,116.50,115.45,113.37,68.08,67.99,50.62,50.36,48.05,47.95$, $44.61,43.98,38.32,37.48,35.88,31.70,31.44,31.01,30.58,29.45,29.39,26.45,26.19$, 26.06, 25.62, 24.12, 23.67, 21.54, 21.47, 13.92, 13.82.

HRMS (ESI-TOF) m/z: [M+H] $]^{+}$calcd for $\mathrm{C}_{40} \mathrm{H}_{41} \mathrm{~N}_{2} \mathrm{O}_{3} 597.3112$; Found 597.3102.<smiles>COC(=O)C(Cc1ccc2c(c1)C(c1ccc(O)cc1)=C(CCCC(=O)NO)CO2)N1C(=O)c2ccccc2C1=O</smiles>

methyl (S)-2-(1,3-dioxoisoindolin-2-yl)-3-(4-(4-methoxyphenyl)-3-(4-oxo-4-(quinolin-8ylamino)butyl)-2H-chromen-6-yl)propanoate (19h)

$40.9 \mathrm{mg}, 60 \%$ yield,yellow sticky liquid.

$\boldsymbol{R}_{\boldsymbol{f}}: 0.40$ (hexane/ethyl acetate, 7:3 v/v).

${ }^{1}$ H NMR (400 MHz, CDCl3) $\delta 9.73$ (s, 1H), 8.80 (d, $\left.J=4.0 \mathrm{~Hz}, 1 \mathrm{H}\right), 8.71$ (d, $J=6.9 \mathrm{~Hz}$, $1 \mathrm{H}), 8.19(\mathrm{~d}, J=7.9 \mathrm{~Hz}, 1 \mathrm{H}), 7.77(\mathrm{dt}, J=6.7,3.4 \mathrm{~Hz}, 3 \mathrm{H}), 7.70(\mathrm{dd}, J=5.4,3.1 \mathrm{~Hz}, 1 \mathrm{H})$, $7.68-7.66(\mathrm{~m}, 1 \mathrm{H}), 7.52(\mathrm{t}, J=7.3 \mathrm{~Hz}, 2 \mathrm{H}), 7.48(\mathrm{dd}, J=8.3,4.3 \mathrm{~Hz}, 1 \mathrm{H}), 7.07$ (d, $J=8.5$ $\mathrm{Hz}, 1 \mathrm{H}), 6.84-6.81(\mathrm{~m}, 1 \mathrm{H}), 6.76(\mathrm{~d}, J=5.4 \mathrm{~Hz}, 1 \mathrm{H}), 6.74-6.69(\mathrm{~m}, 1 \mathrm{H}), 6.64(\mathrm{~d}, J=8.1$ $\mathrm{Hz}, 1 \mathrm{H}), 6.36(\mathrm{~d}, J=1.7 \mathrm{~Hz}, 1 \mathrm{H}), 4.91(\mathrm{dd}, J=11.0,5.3 \mathrm{~Hz}, 1 \mathrm{H}), 4.74(\mathrm{~s}, J=14.4 \mathrm{~Hz}, 2 \mathrm{H})$, $3.78(\mathrm{~s}, 3 \mathrm{H}), 3.71(\mathrm{~s}, J=5.7 \mathrm{~Hz}, 3 \mathrm{H}), 3.53-3.47(\mathrm{~m}, 1 \mathrm{H}), 3.33-3.25(\mathrm{~m}, 1 \mathrm{H}), 2.44(\mathrm{t}, J=$ $7.4 \mathrm{~Hz}, 2 \mathrm{H}), 2.13-2.02(\mathrm{~m}, 2 \mathrm{H}), 1.83(\mathrm{dt}, J=15.0,7.5 \mathrm{~Hz}, 2 \mathrm{H})$.

${ }^{13}$ C NMR (100 MHz, CDCl $) \delta 169.45,167.59,167.40,158.71,152.32,134.22,134.17$, $131.74,131.70,131.53,130.32$, 130.11, 130.08, 129.38, 128.69, 128.53, 128.14, 127.72, $126.40,125.56,123.62,123.59,121.68,121.64,115.66,115.61,115.05,68.18,55.24,53.65$, $52.89,37.49,34.02,30.60,23.67$.

HRMS (ESI-TOF) m/z: [M+H $]^{+}$calcd for $\mathrm{C}_{41} \mathrm{H}_{36} \mathrm{~N}_{3} \mathrm{O}_{7}$ 682.2548; Found 682.2555. 
<smiles></smiles>

\section{methyl $(S)$-4-phenyl-4-(2-phenyl-1-tosyl-1H-indol-3-yl)butanoate (22a)}

$48.2 \mathrm{mg}, 92 \%$ yield, white solid.

$\boldsymbol{R}_{\boldsymbol{f}}: 0.40$ (hexane/ethyl acetate, $7: 3 \mathrm{v} / \mathrm{v}$ ).

${ }^{1}$ H NMR (500 MHz, CDCl $) \delta 8.41(\mathrm{~d}, J=8.3 \mathrm{~Hz}, 1 \mathrm{H}), 7.53-7.41(\mathrm{~m}, 4 \mathrm{H}), 7.37$ (t, $J=7.6$ $\mathrm{Hz}, 4 \mathrm{H}), 7.28-7.17(\mathrm{~m}, 5 \mathrm{H}), 7.12(\mathrm{~m}, 4 \mathrm{H}), 4.05(\mathrm{dd}, J=10.7,5.5 \mathrm{~Hz}, 1 \mathrm{H}), 3.52(\mathrm{~s}, 3 \mathrm{H})$, $2.57-2.42(\mathrm{~m}, 2 \mathrm{H}), 2.38(\mathrm{~s}, 3 \mathrm{H}), 2.10-2.05(\mathrm{~m}, 2 \mathrm{H})$.

${ }^{13}$ C NMR (125 MHz, CDCl3) $\delta 173.39,144.65,142.61,138.48,138.05,135.04,132.11$, $131.21,130.66,129.34,129.31,128.85,128.32,127.68,127.31,126.81,126.27,124.93$, $124.71,123.99,121.06,116.60,51.44,40.20,32.24,27.83,21.61$.

HRMS (ESI-TOF) m/z: [M+H] $]^{+}$calcd for $\mathrm{C}_{32} \mathrm{H}_{30} \mathrm{NO}_{4} \mathrm{~S}$ 524.1890; Found 524.1900.

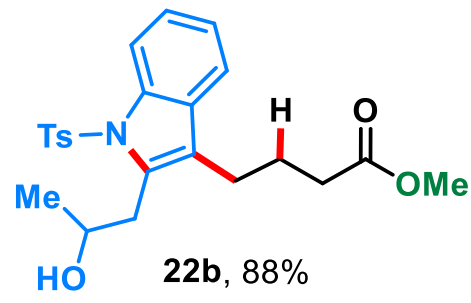

\section{methyl 4-(2-(2-hydroxypropyl)-1-tosyl-1H-indol-3-yl)butanoate (22b)}

$37.8 \mathrm{mg}, 88 \%$ yield, yellow sticky liquid.

$\boldsymbol{R}_{f}: 0.40$ (hexane/ethyl acetate, $7: 3 \mathrm{v} / \mathrm{v}$ ).

${ }^{1}$ H NMR (400 MHz, CDCl3) $\delta 8.15(\mathrm{dd}, \mathrm{J}=7.3,1.2 \mathrm{~Hz}, 1 \mathrm{H}), 7.49(\mathrm{~d}, \mathrm{~J}=8.4 \mathrm{~Hz}, 2 \mathrm{H}), 7.46$ - $7.40(\mathrm{~m}, 1 \mathrm{H}), 7.31-7.20(\mathrm{~m}, 2 \mathrm{H}), 7.12(\mathrm{~d}, \mathrm{~J}=8.1 \mathrm{~Hz}, 2 \mathrm{H}), 4.37-4.22(\mathrm{~m}, 1 \mathrm{H}), 3.63(\mathrm{~s}$, 3H), $3.20(\mathrm{dd}, \mathrm{J}=14.5,4.2 \mathrm{~Hz}, 1 \mathrm{H}), 3.01(\mathrm{dd}, \mathrm{J}=14.5,7.9 \mathrm{~Hz}, 1 \mathrm{H}), 2.74-2.62(\mathrm{~m}, 2 \mathrm{H})$, $2.29(\mathrm{~s}, 3 \mathrm{H}), 2.22$ (t, J = 7.2 Hz, 2H), $1.94-1.79$ (m, 2H), $1.30(\mathrm{~d}, \mathrm{~J}=6.2 \mathrm{~Hz}, 3 \mathrm{H})$.

${ }^{13}$ C NMR (100 MHz, CDCl3) $\delta$ 173.95, 144.72, 137.15, 135.47, 134.40, 130.78, 129.77, $126.21,124.58,123.86,123.29,118.95,115.72$, 68.30, 51.67, 36.49, 33.24, 24.80, 23.74, 23.18, 21.60 .

HRMS (ESI-TOF) m/z: [M+H] ${ }^{+}$calcd for $\mathrm{C}_{23} \mathrm{H}_{28} \mathrm{NO}_{5} \mathrm{~S} 430.1683$; Found 430.1687. 
<smiles>CC(C)(C)OCc1c(CCC(N)=O)c2ccccc2n1Cc1ccccc1</smiles>

\section{4-(2-(((1-methyl-4-oxocyclohexa-2,5-dien-1-yl)oxy)methyl)-1-tosyl-1H-indol-3-yl)- $N$ -} (quinolin-8-yl)butanamide (24)

$43.4 \mathrm{mg}, 70 \%$ yield, white solid.

$\boldsymbol{R}_{\boldsymbol{f}}: 0.40$ (hexane/ethyl acetate, $6: 4 \mathrm{v} / \mathrm{v}$ ).

${ }^{1}$ H NMR (500 MHz, CDCl 3$) \delta 9.78(\mathrm{~s}, 1 \mathrm{H}), 8.87-8.76(\mathrm{~m}, 2 \mathrm{H}), 8.20(\mathrm{~d}, J=8.2 \mathrm{~Hz}, 1 \mathrm{H})$, $8.11(\mathrm{~d}, J=8.4 \mathrm{~Hz}, 1 \mathrm{H}), 7.85(\mathrm{~d}, J=8.3 \mathrm{~Hz}, 2 \mathrm{H}), 7.61-7.53(\mathrm{~m}, 3 \mathrm{H}), 7.48(\mathrm{dd}, J=8.2,4.2$ $\mathrm{Hz}, 1 \mathrm{H}), 7.33$ (t, $J=7.7 \mathrm{~Hz}, 1 \mathrm{H}), 7.24(\mathrm{t}, J=7.5 \mathrm{~Hz}, 1 \mathrm{H}), 7.18$ (d, $J=8.1 \mathrm{~Hz}, 2 \mathrm{H}), 7.06$ (d, $J$ $=10.1 \mathrm{~Hz}, 2 \mathrm{H}), 6.31(\mathrm{~d}, J=10.1 \mathrm{~Hz}, 2 \mathrm{H}), 4.73(\mathrm{~s}, 2 \mathrm{H}), 2.86(\mathrm{t}, J=7.5 \mathrm{~Hz}, 2 \mathrm{H}), 2.55(\mathrm{t}, J=$ $6.9 \mathrm{~Hz}, 2 \mathrm{H}), 2.29$ (s, 3H), $2.19-2.12(\mathrm{~m}, 2 \mathrm{H}), 1.45$ (s, 3H).

${ }^{13}$ C NMR (125 MHz, CDCl3) $\delta 185.25,171.10,152.05,148.26,144.64,138.29,136.62$, $134.41,132.85,130.33,129.69,129.58,128.08,127.53,126.88,125.54,125.07,123.60$, $121.78,119.66,116.47,115.20,72.87,57.40,32.02,29.75,29.46,22.79,14.22$.

HRMS (ESI-TOF) m/z: [M+H] $]^{+}$calcd for $\mathrm{C}_{36} \mathrm{H}_{34} \mathrm{~N}_{3} \mathrm{O}_{5} \mathrm{~S}$ 620.2214; Found 620.2216.

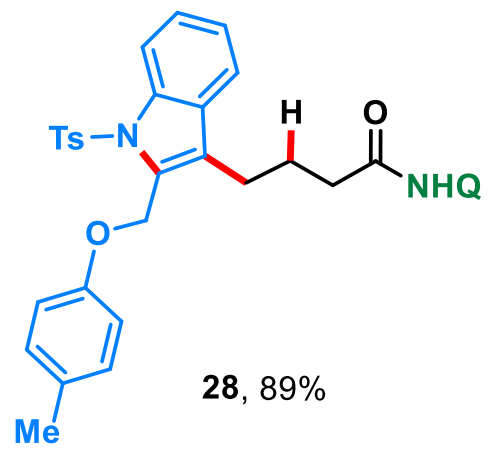

$\underline{\mathrm{N} \text {-(quinolin-8-yl)-4-(2-((p-tolyloxy)methyl)-1-tosyl-1H-indol-3-yl)butanamide (28) }}$

$53.7 \mathrm{mg}, 89 \%$ yield, white solid.

$\boldsymbol{R}_{f}: 0.40$ (hexane/ethyl acetate, $6: 4 \mathrm{v} / \mathrm{v}$ ).

${ }^{1}$ H NMR (500 MHz, CDCl3) $\delta 9.73(\mathrm{~s}, 1 \mathrm{H}), 8.81$ (dd, $\left.J=4.2,1.6 \mathrm{~Hz}, 1 \mathrm{H}\right), 8.76(\mathrm{dd}, J=7.0$, $1.8 \mathrm{~Hz}, 1 \mathrm{H}), 8.23(\mathrm{~d}, J=8.4 \mathrm{~Hz}, 1 \mathrm{H}), 8.19(\mathrm{dd}, J=8.3,1.5 \mathrm{~Hz}, 1 \mathrm{H}), 7.86(\mathrm{~d}, J=8.4 \mathrm{~Hz}, 2 \mathrm{H})$, $7.62(\mathrm{~d}, J=7.7 \mathrm{~Hz}, 1 \mathrm{H}), 7.58-7.51(\mathrm{~m}, 2 \mathrm{H}), 7.48(\mathrm{dd}, J=8.2,4.2 \mathrm{~Hz}, 1 \mathrm{H}), 7.38$ (ddd, $J=$ 
8.4, 7.3, $1.2 \mathrm{~Hz}, 1 \mathrm{H}), 7.28-7.24(\mathrm{~m}, 1 \mathrm{H}), 7.19(\mathrm{~d}, J=8.2 \mathrm{~Hz}, 2 \mathrm{H}), 7.03(\mathrm{~d}, J=8.3 \mathrm{~Hz}, 2 \mathrm{H})$, $6.81(\mathrm{~d}, J=8.5 \mathrm{~Hz}, 2 \mathrm{H}), 5.40(\mathrm{~s}, 2 \mathrm{H}), 2.91(\mathrm{t}, J=7.3 \mathrm{~Hz}, 2 \mathrm{H}), 2.53(\mathrm{t}, J=7.2 \mathrm{~Hz}, 2 \mathrm{H}), 2.30$ (s, 3H), $2.28(\mathrm{~s}, 3 \mathrm{H}), 2.14$ (p, $J=7.3 \mathrm{~Hz}, 2 \mathrm{H})$.

${ }^{13}$ C NMR (125 MHz, CDCl3) $\delta 171.18,156.30,148.25,144.67,138.39,136.85,136.48$, $136.05,134.53,131.67,130.48,130.03,129.71,129.56,128.07,127.55,127.25,125.62$, $125.08,123.58,121.75,121.54,119.86,116.58,115.18,114.80,60.19,36.73,25.33,23.54$, 21.63, 20.62.

HRMS (ESI-TOF) m/z: [M+H] $]^{+}$calcd for $\mathrm{C}_{36} \mathrm{H}_{34} \mathrm{~N}_{3} \mathrm{O}_{4} \mathrm{~S}$ 604.2265; Found 604.2250.

\section{References.}

1) (a) Gurak, J. A., Jr.; Yang, K. S.; Liu, Z.; Engle, K. M. J. Am. Chem. Soc. 2016, 138, 5805. (b) Yang, K. S.; Gurak, J. A., Jr.; Liu, Z.; Engle, K. M. J. Am. Chem. Soc. 2016, 138, 14705.

2) Vogel, A. I. A Text Book of Practical Organic Chemistry, 4th ed.; ELBS, Longman Group Limited: London, 1978; p 658. 
9. ${ }^{1} \mathrm{H}$ and ${ }^{13} \mathrm{C}$ NMR Spectra of the Compounds

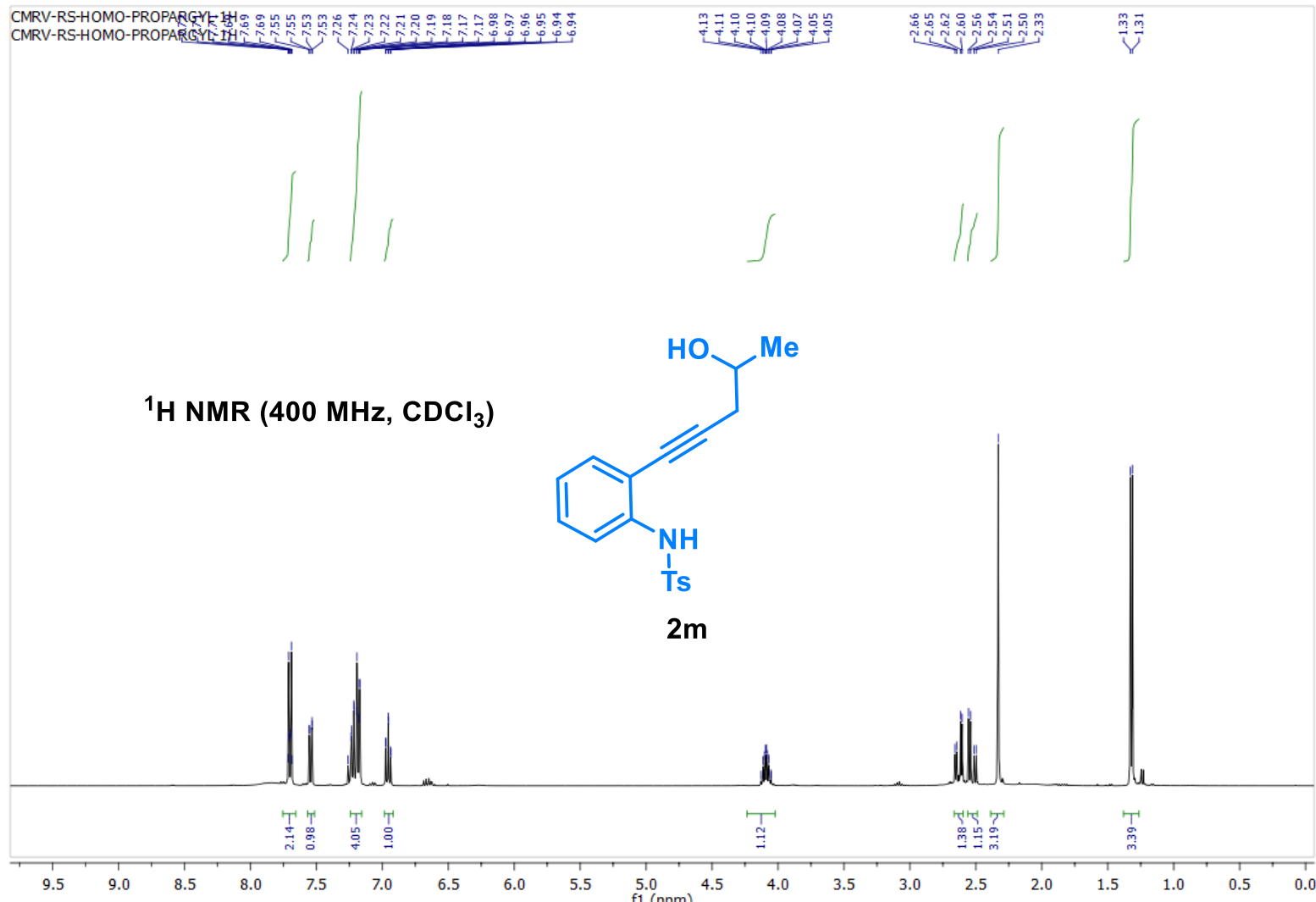

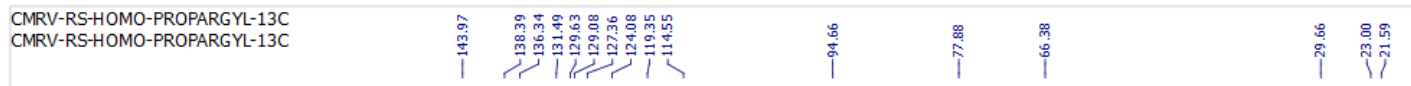

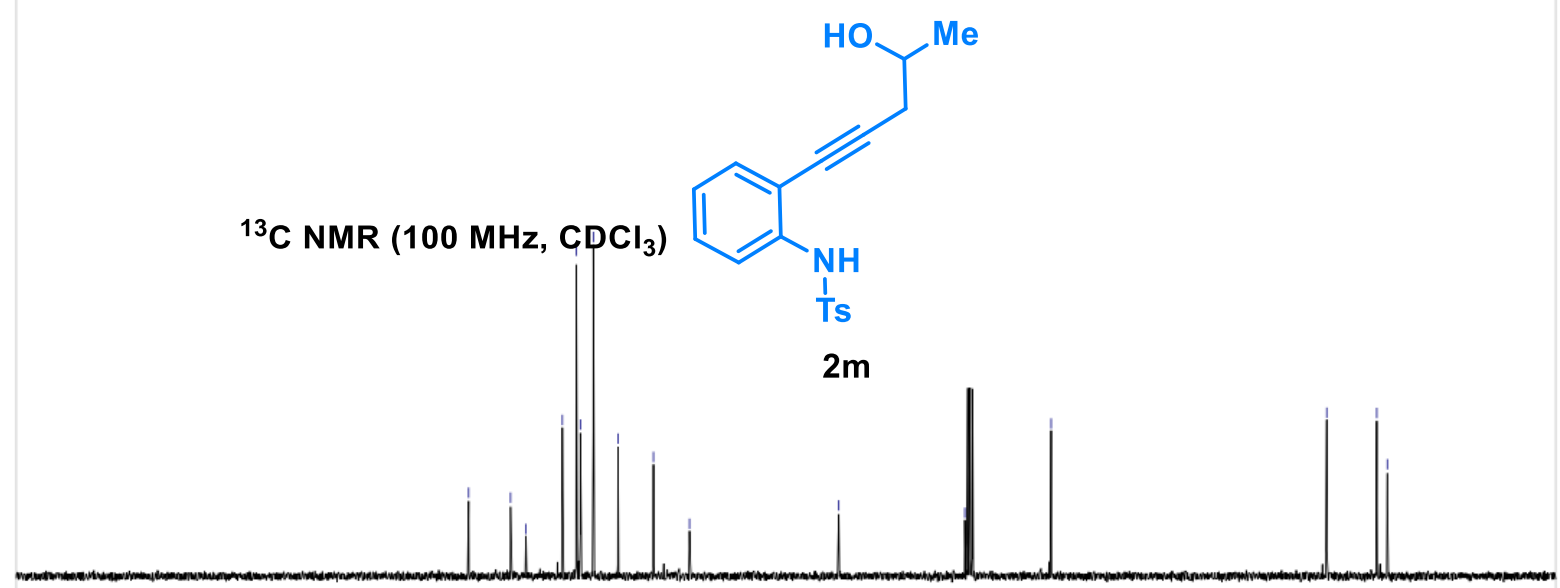

$\begin{array}{llllllllll}200 & 190 & 180 & 170 & 160 & 150 & 140 & 130 & 120 & 110 \\ \mathrm{f} 1(\mathrm{ppm})\end{array}$ 


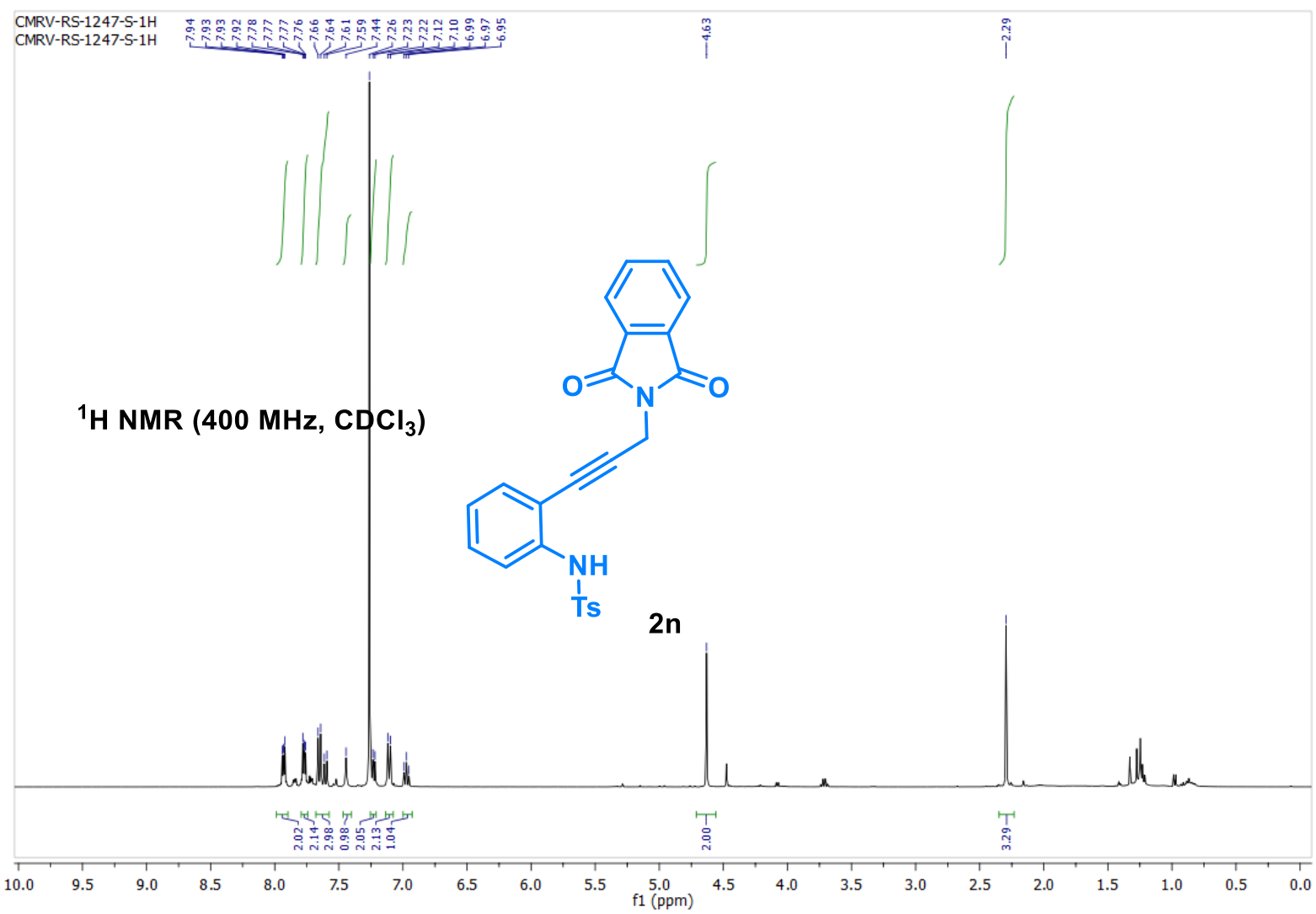
CMRV-RS-1247-S-13C
CMRV-RS-1247-S-13C

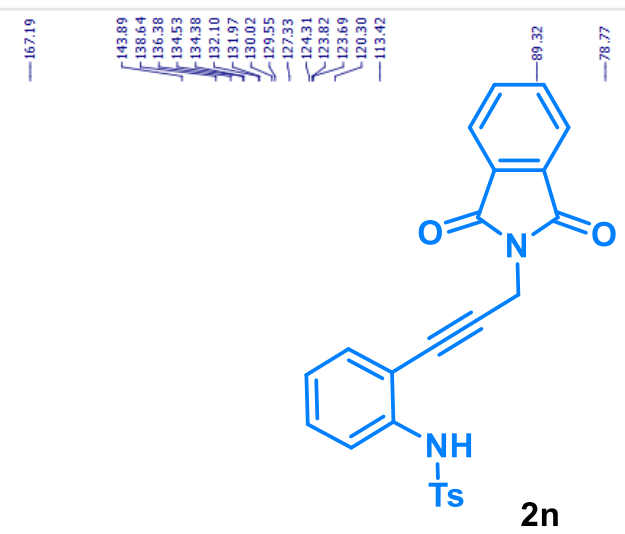

${ }^{13} \mathrm{C}$ NMR (125 MHz, $\left.\mathrm{CDCl}_{3}\right)$

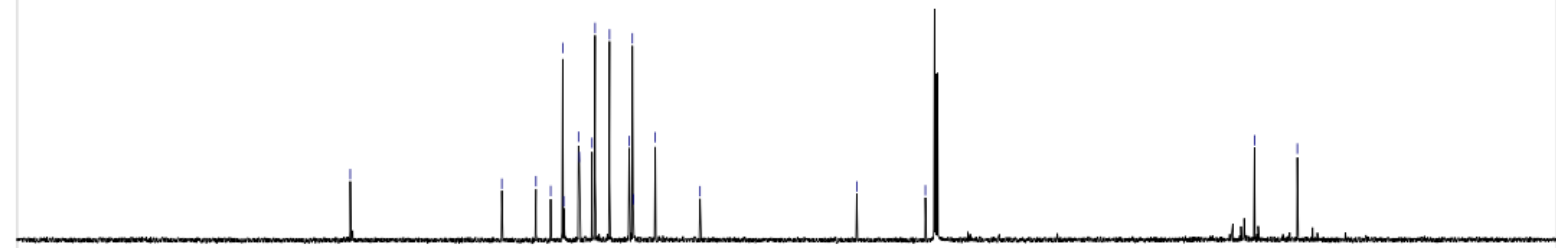

$\begin{array}{llllllllllllllllllllllllllll} & 210 & 200 & 190 & 180 & 170 & 160 & 150 & 140 & 130 & 120 & 110 & 100 & 90 & 80 & 70 & 60 & 50 & 40 & 30 & 20 & 10 & 0 & -10\end{array}$ 

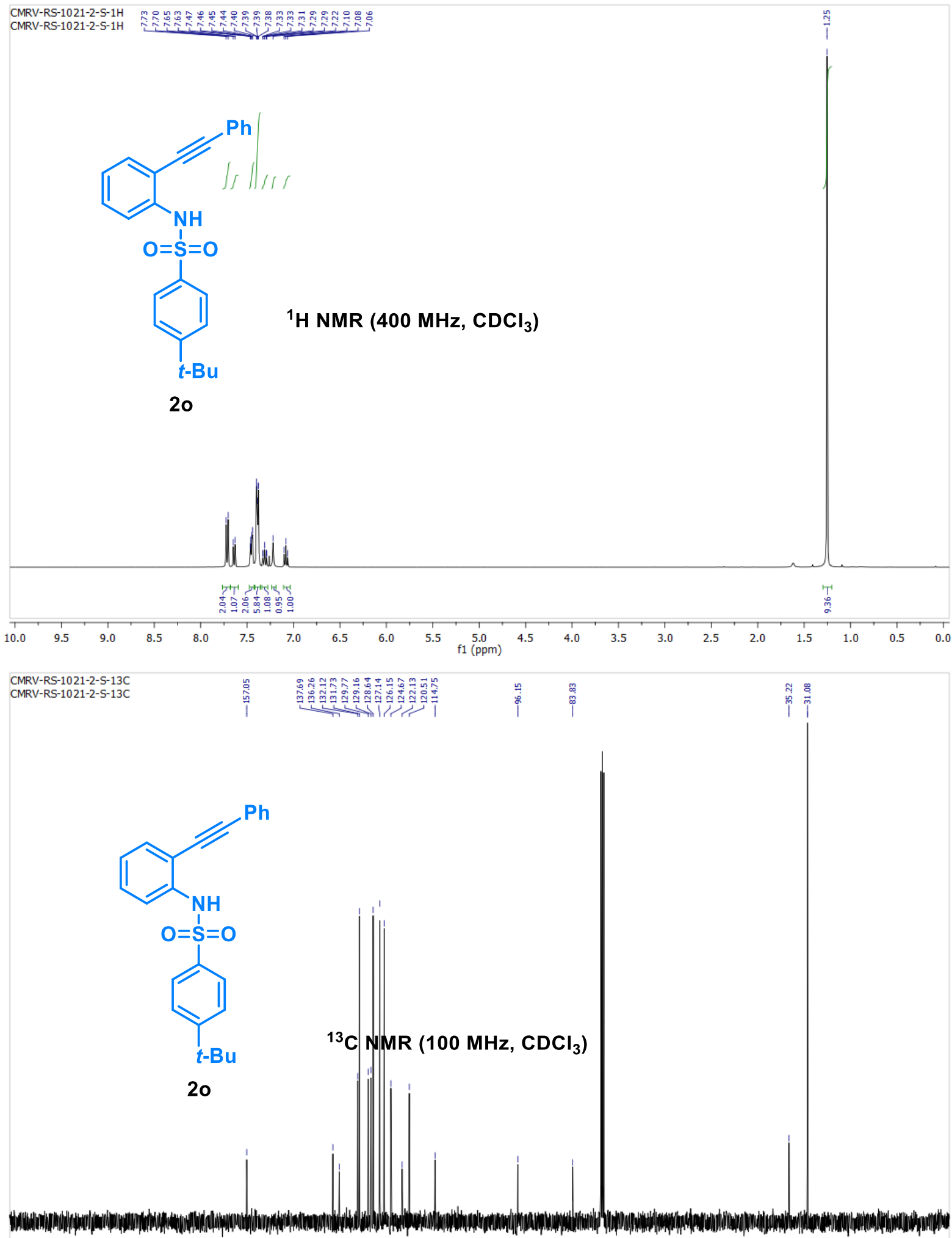

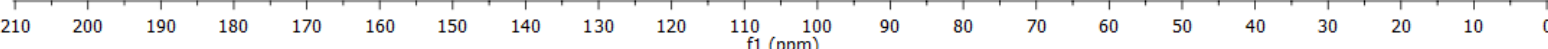




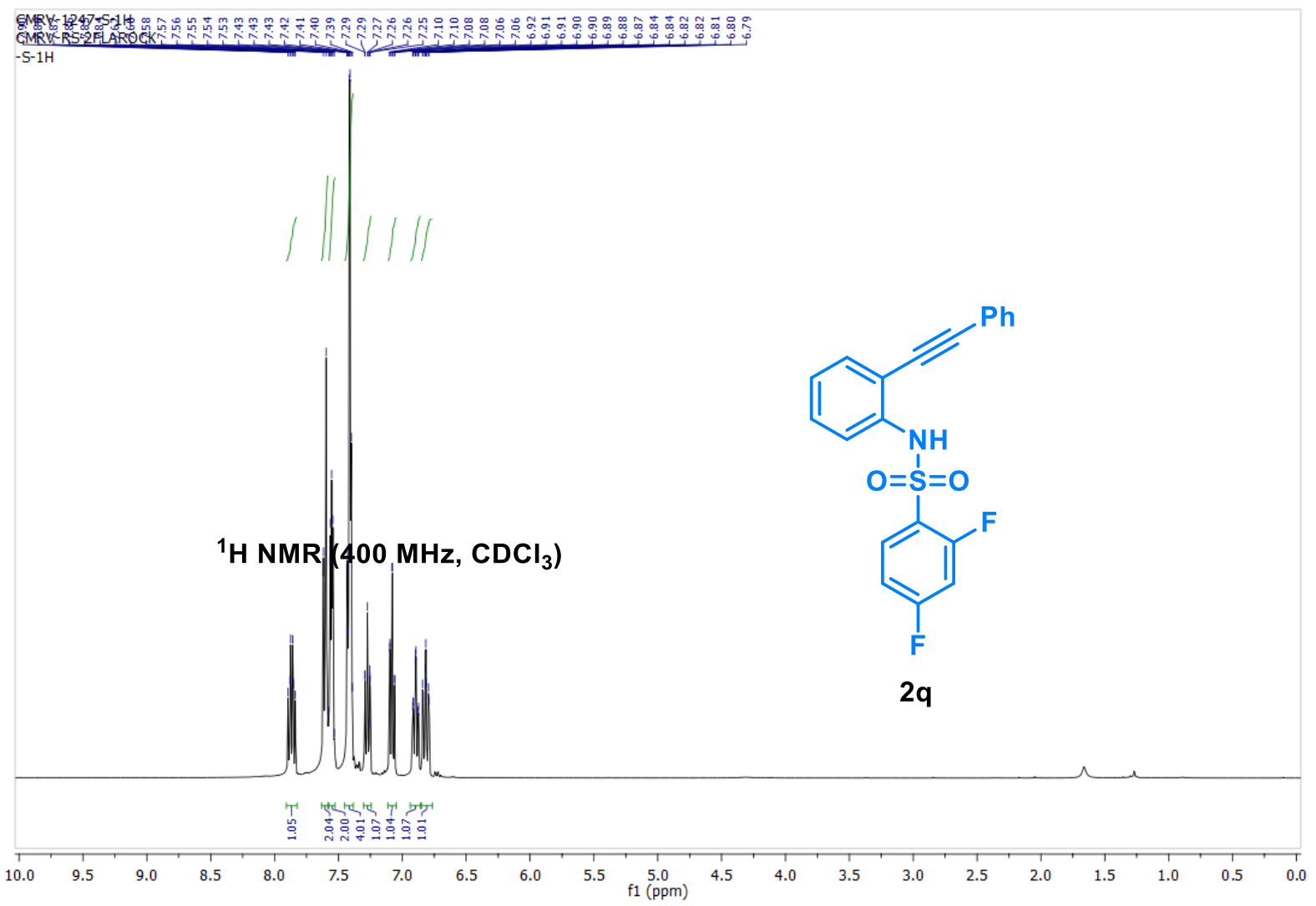

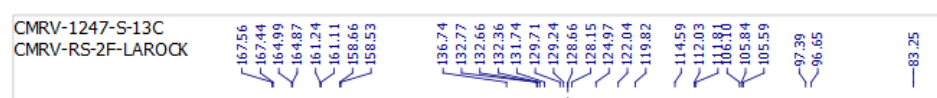

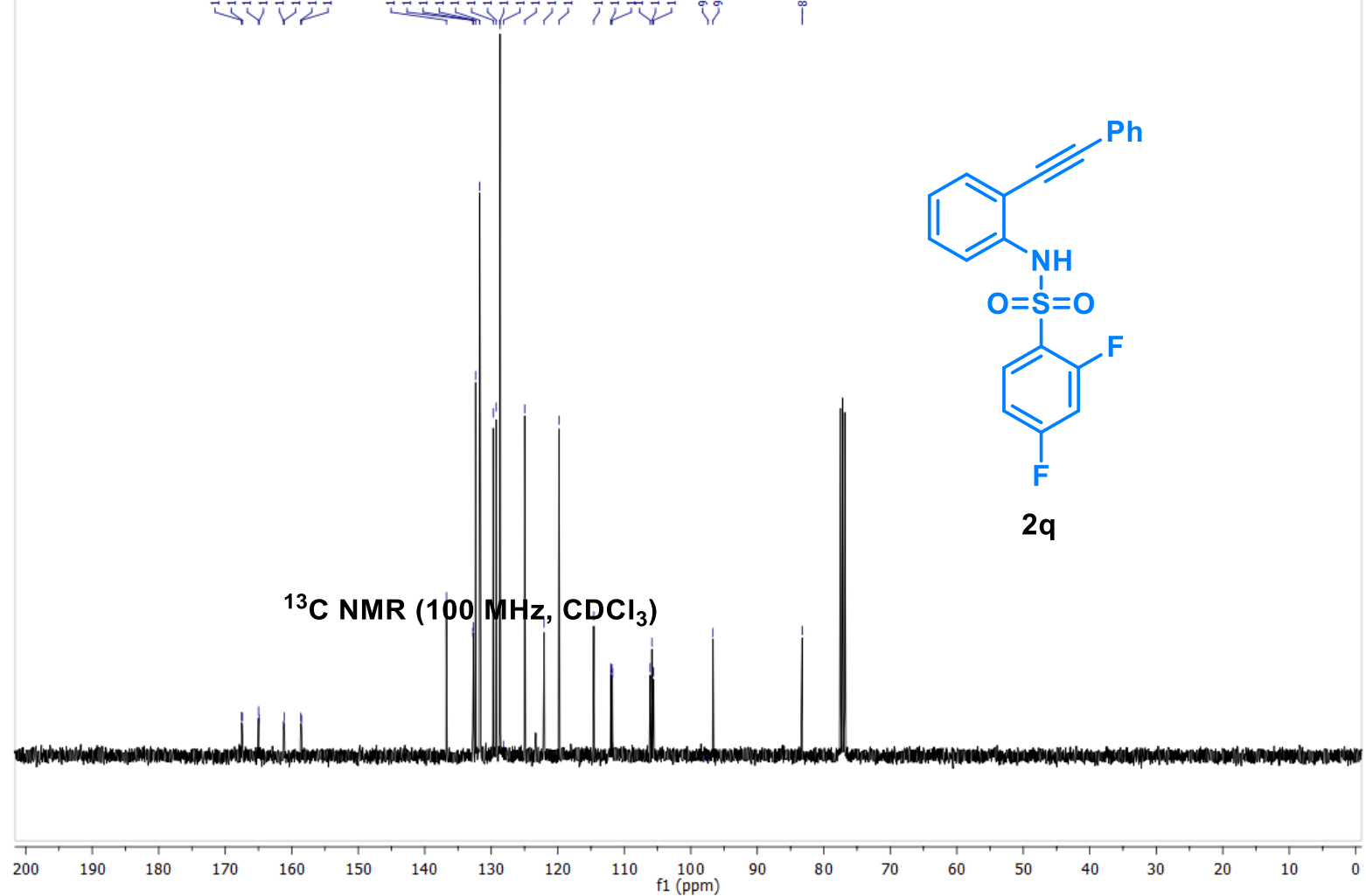



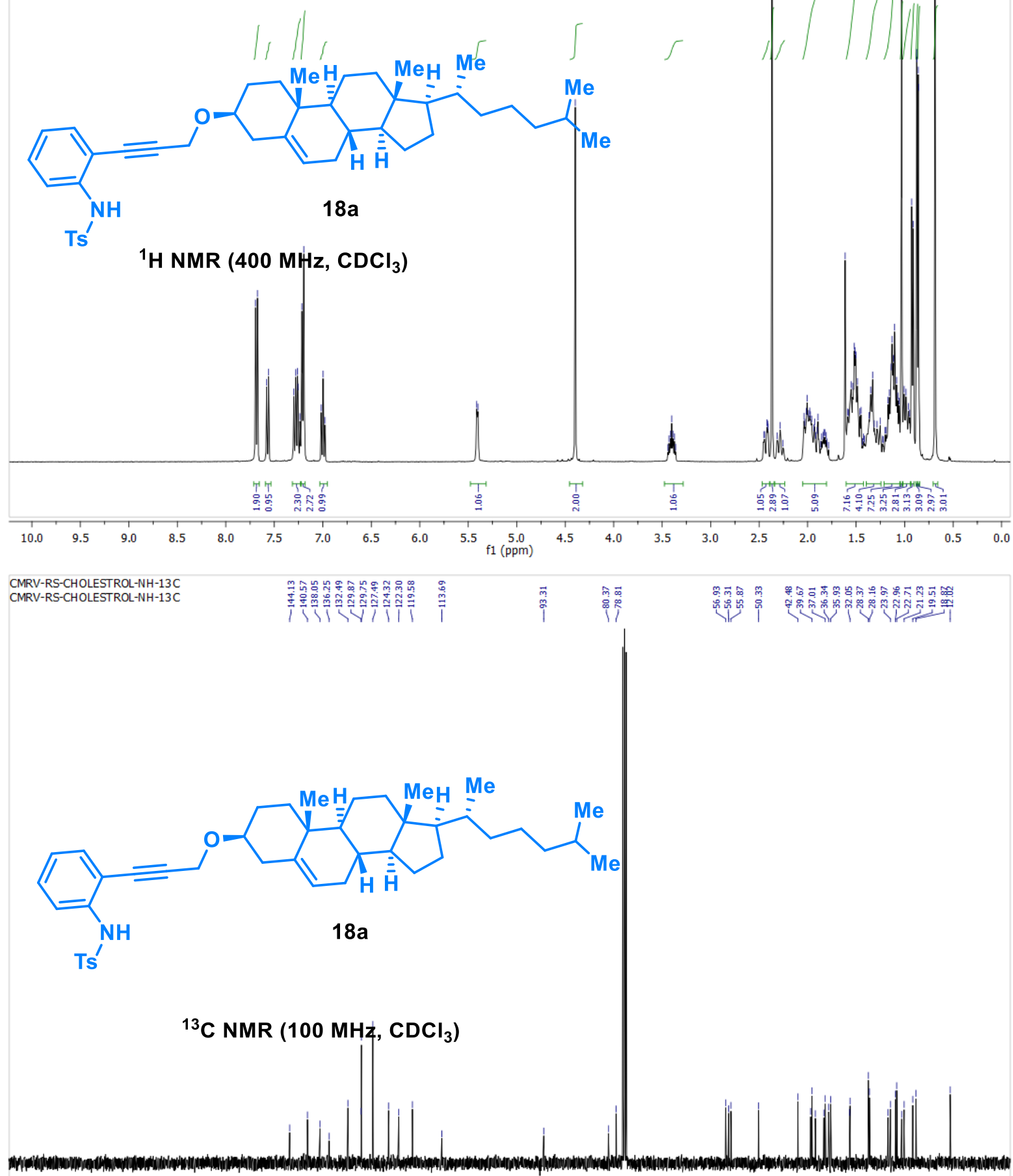
CMRV-RS-ETHYSTRONE-NH-1H
CMRV-RS-ETHYSTRONE-NH-1H
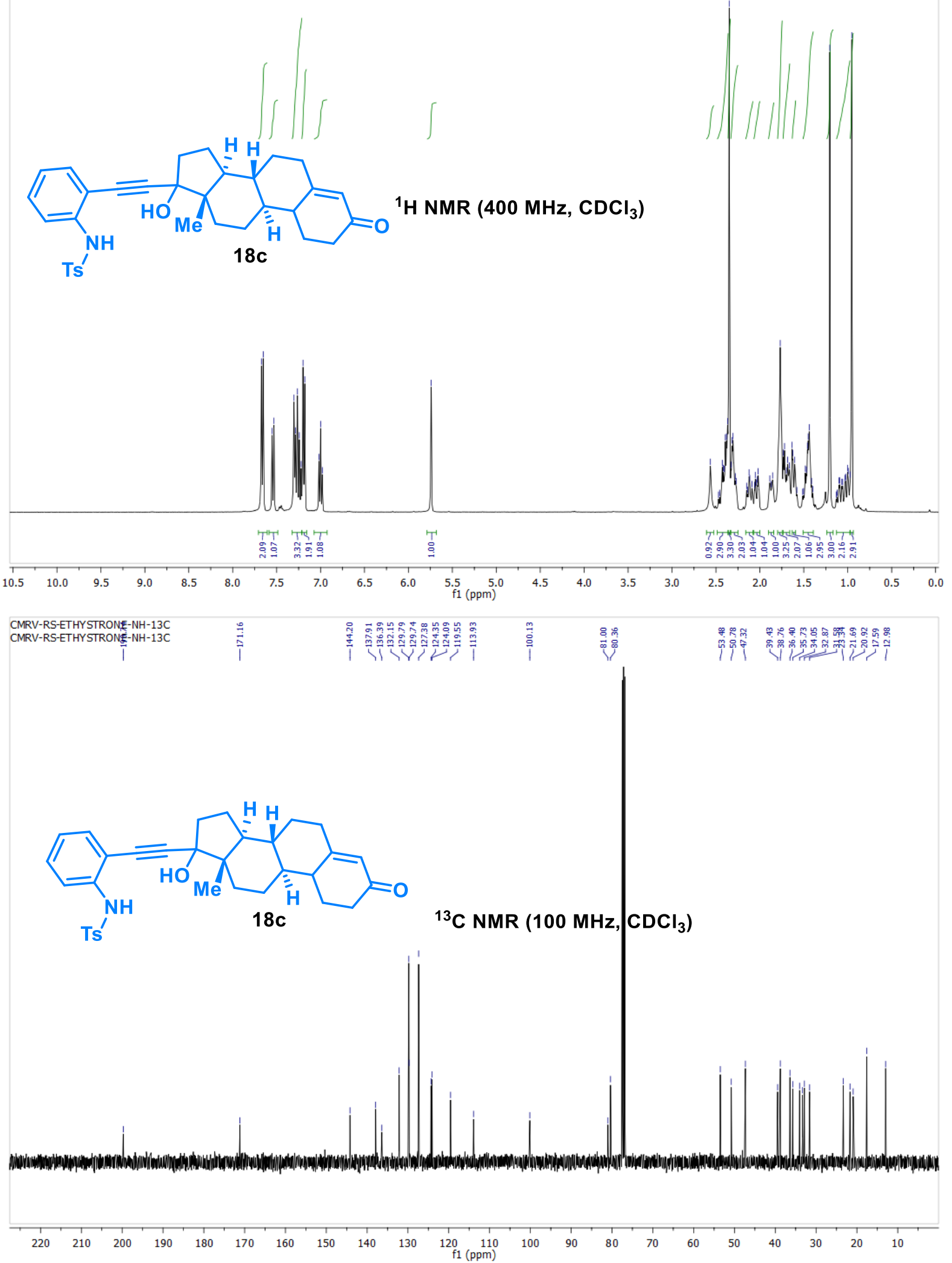


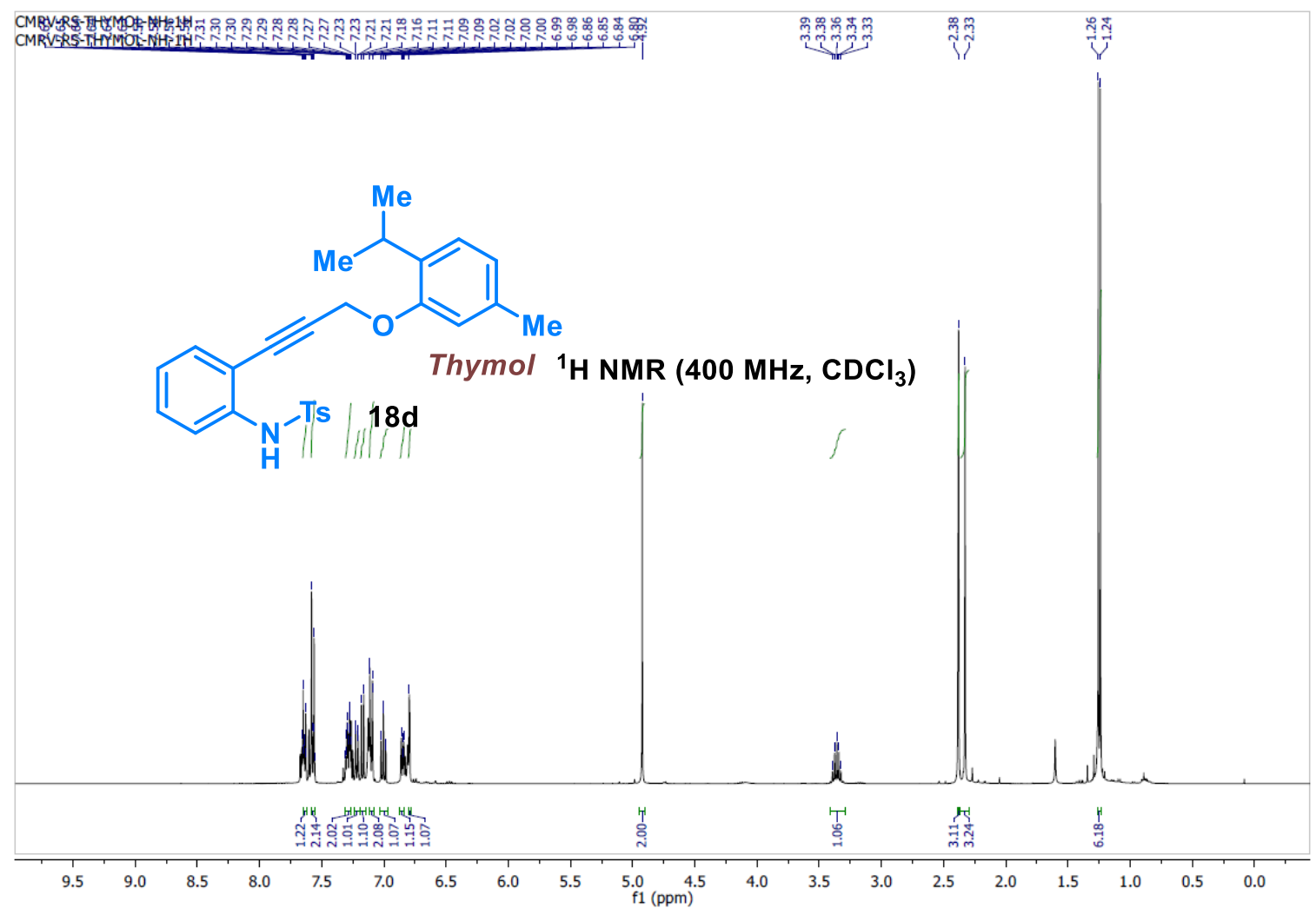

\begin{tabular}{|c|c|c|c|c|}
\hline $\begin{array}{l}\text { CMRV-RS-THYMOL-NH-13C } \\
\text { CMRV-RS-THYMOL-NH-13C }\end{array}$ & 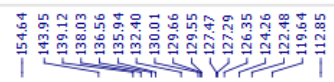 & $\stackrel{\circ}{i}$ & 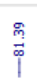 & 㞻 \\
\hline
\end{tabular}<smiles>COc1ccc(C(C)C)c(OCC#Cc2ccccc2N[As])c1</smiles>
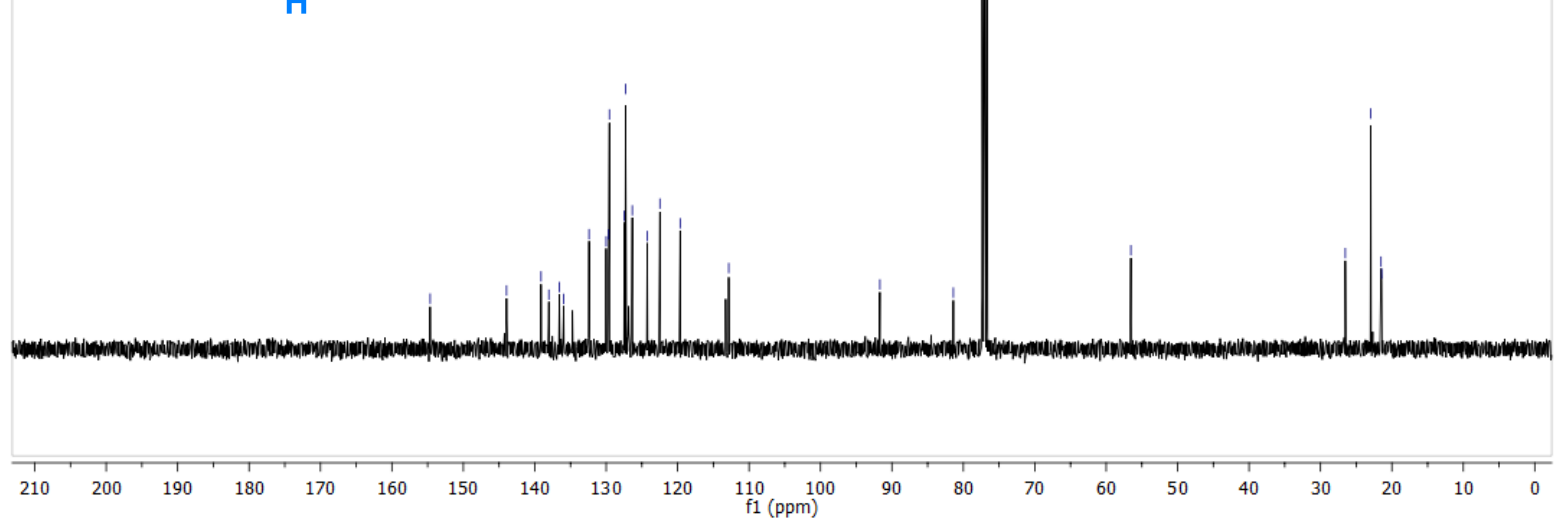

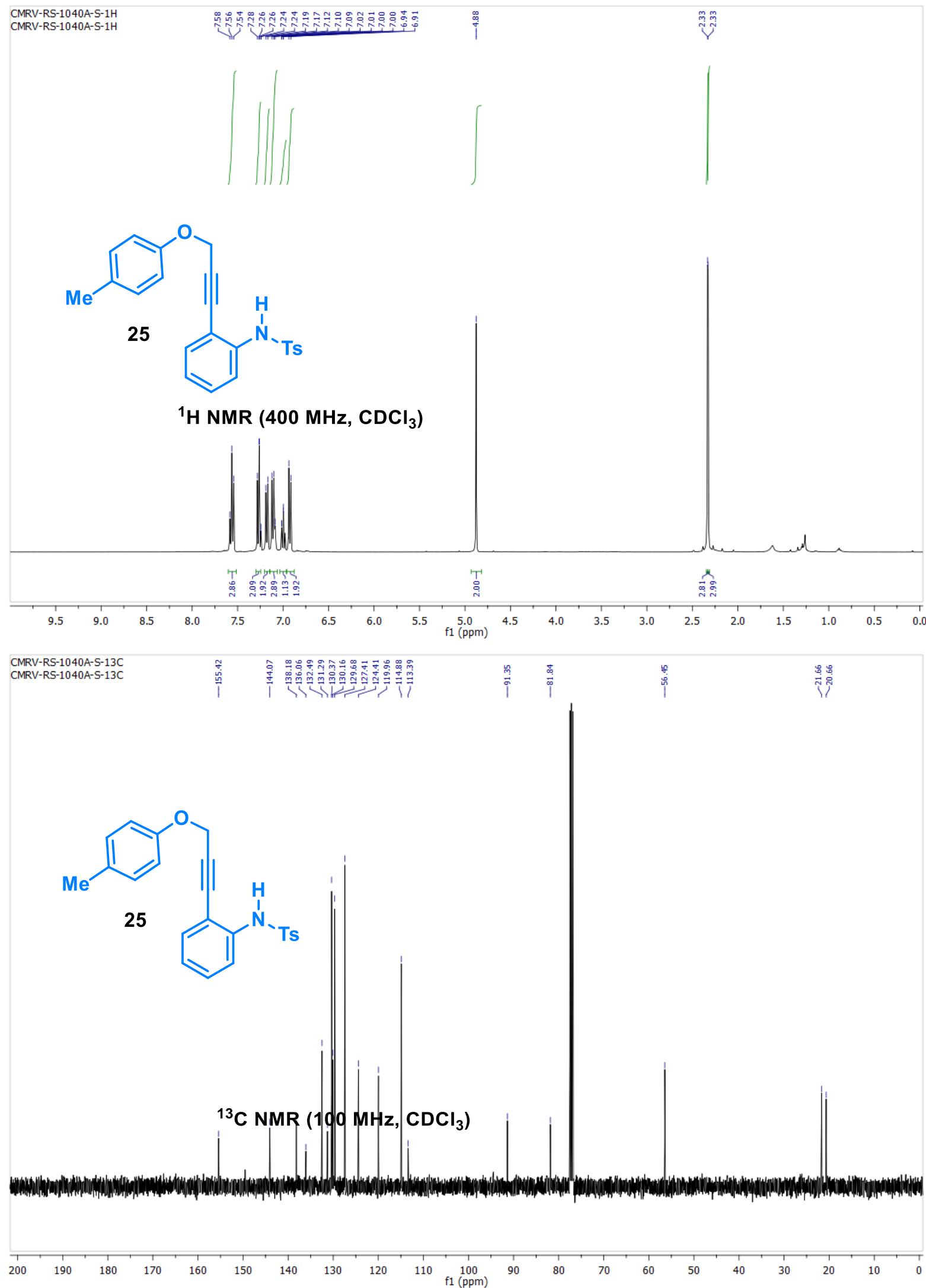


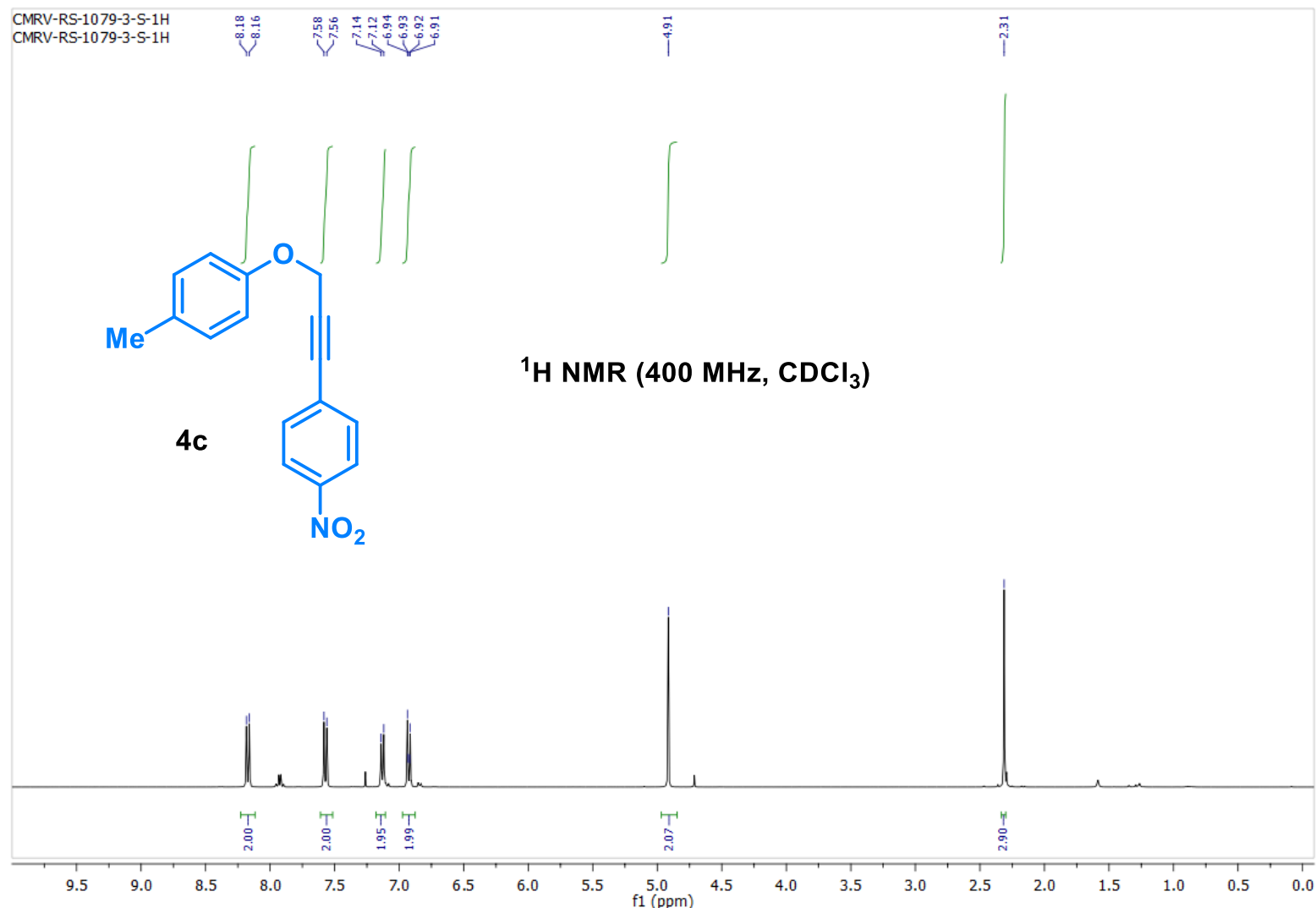

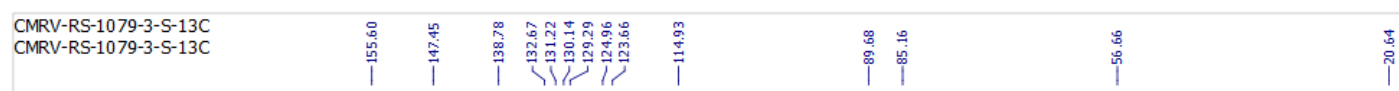

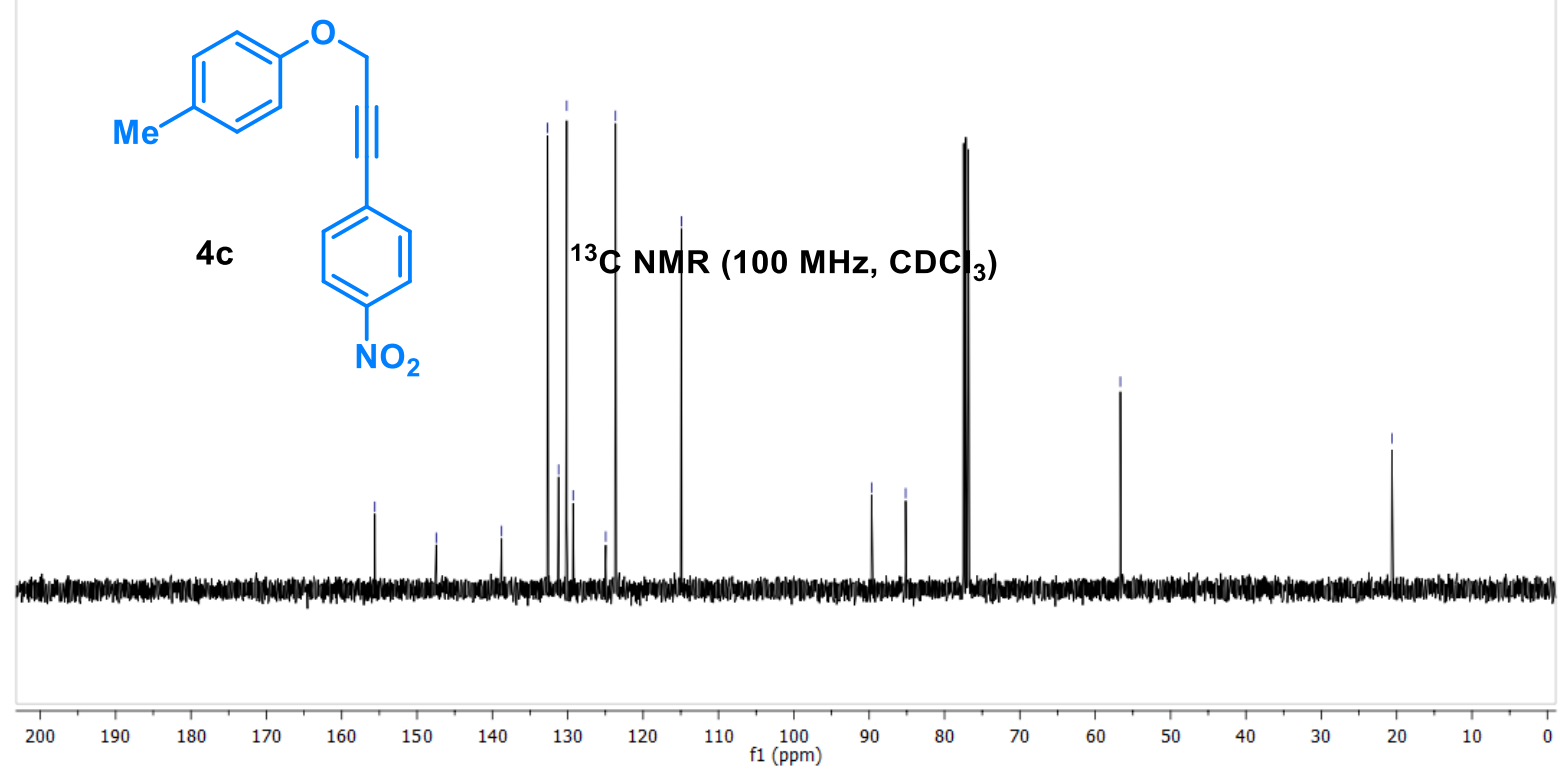



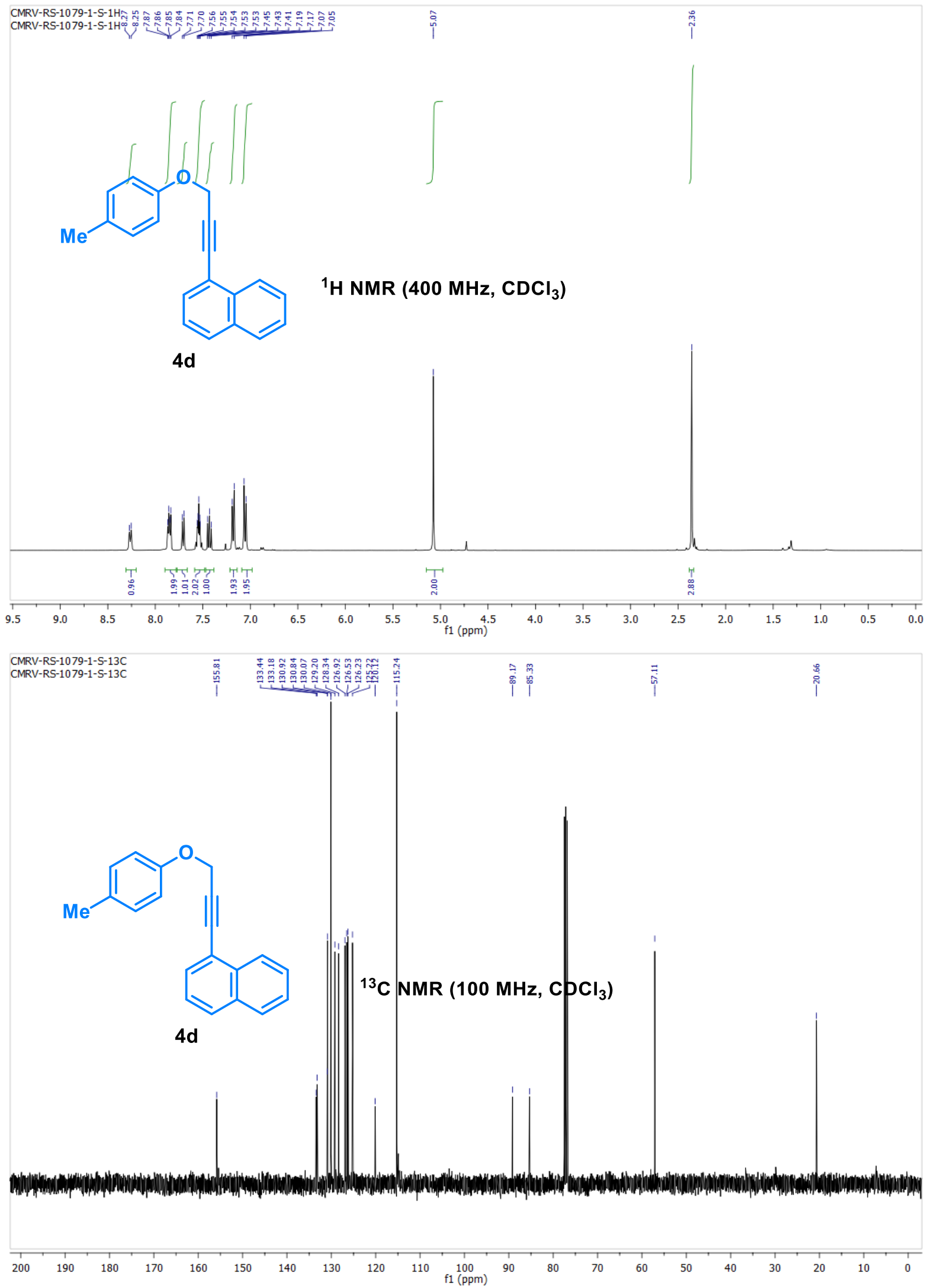


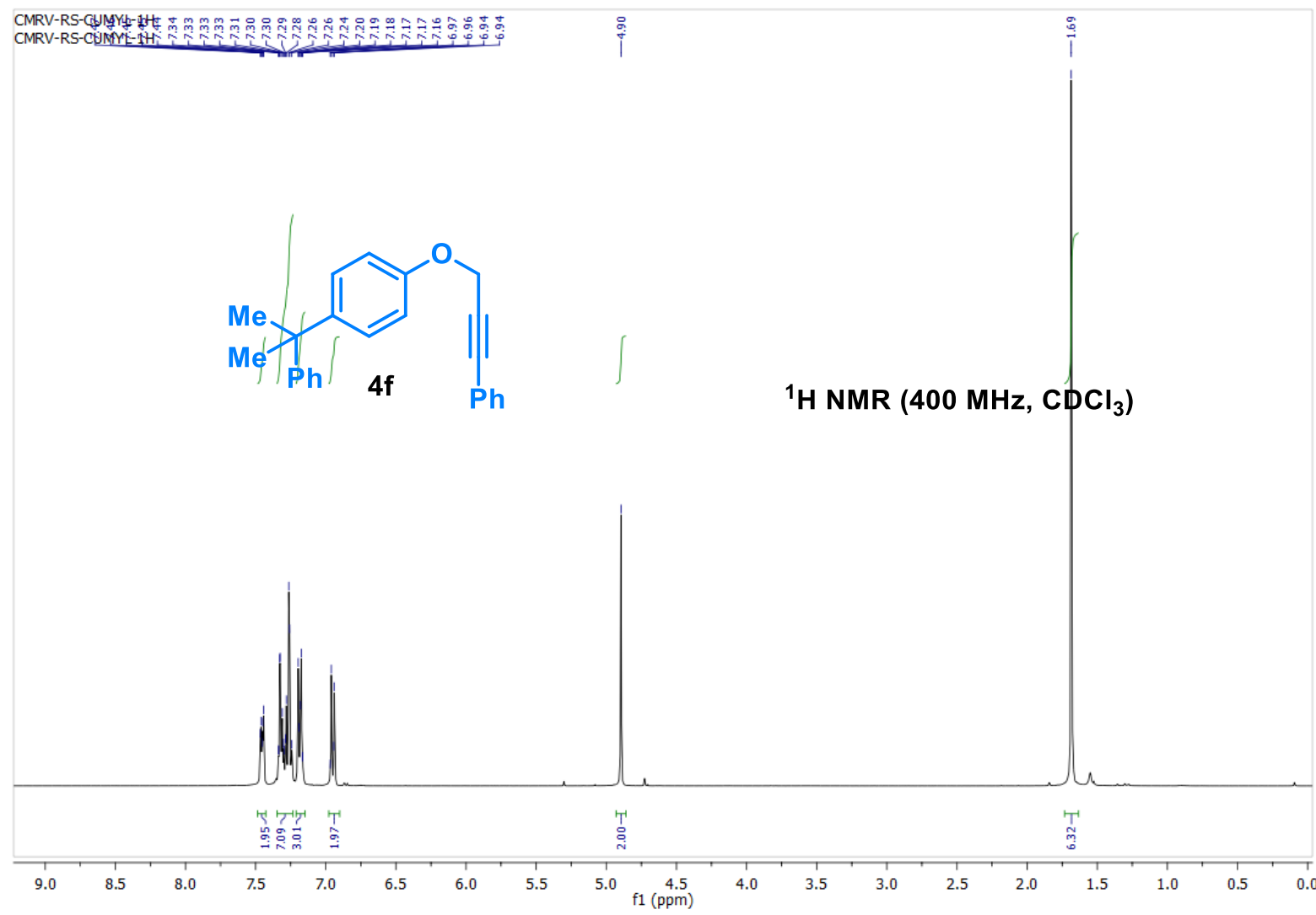

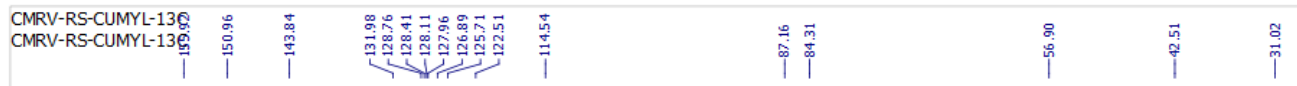<smiles>CC(C)(c1ccccc1)c1ccc(OCC#CPc2ccccc2)cc1</smiles>
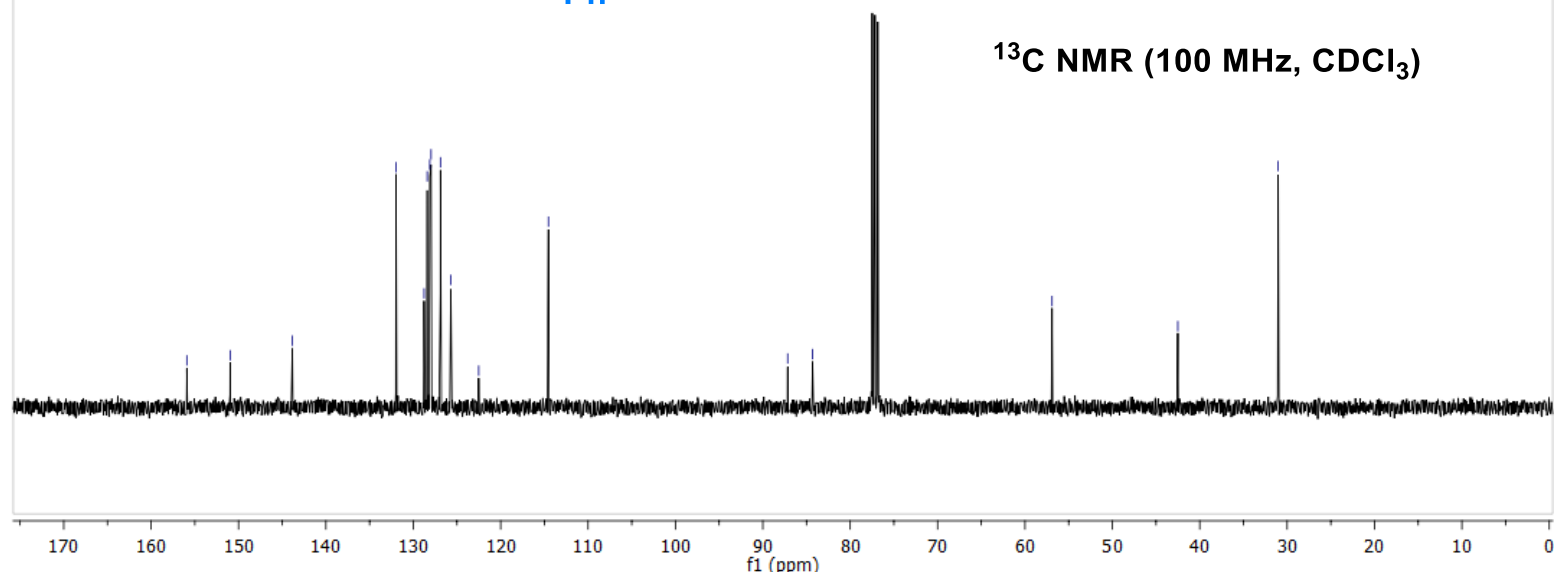

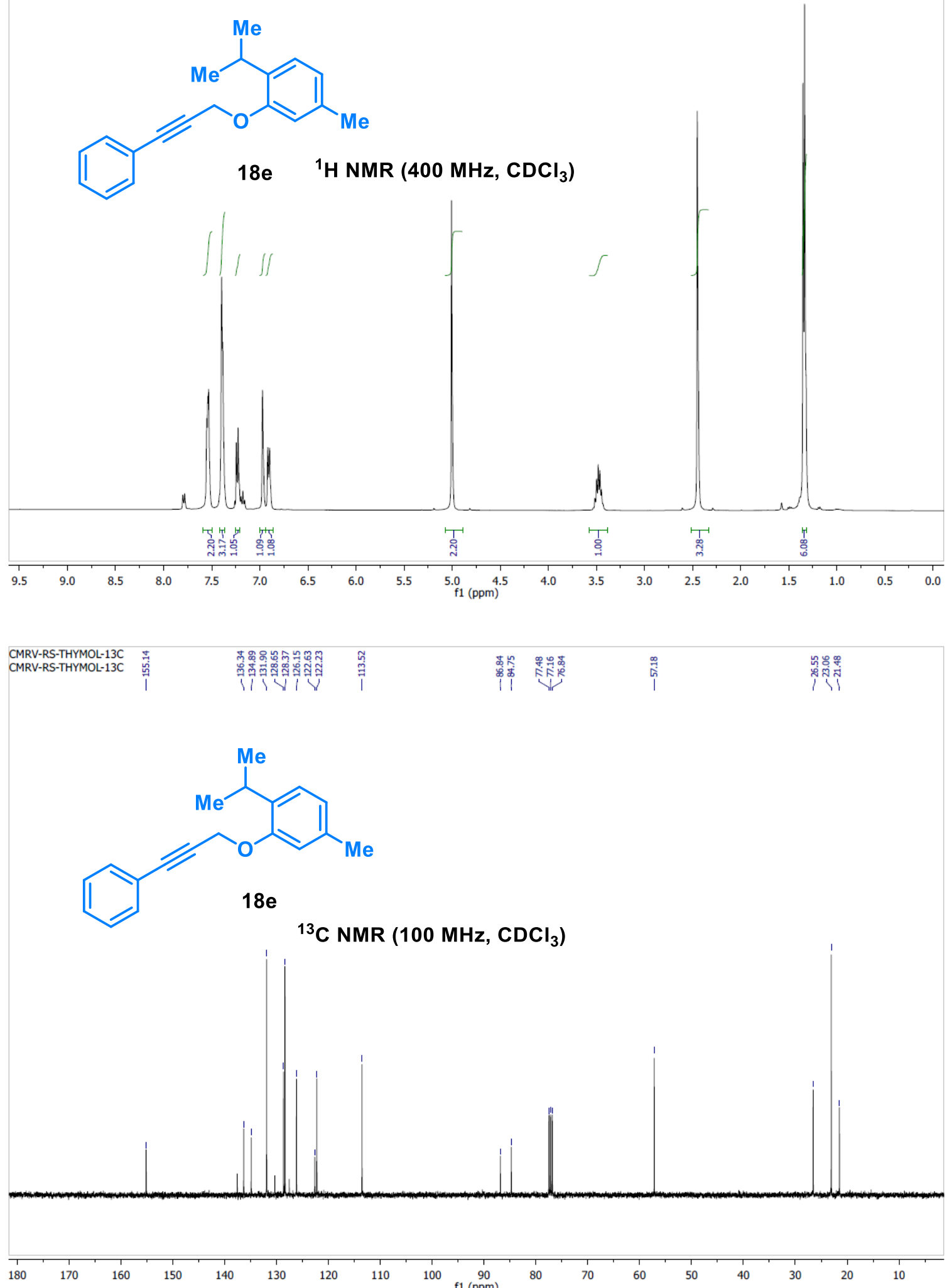

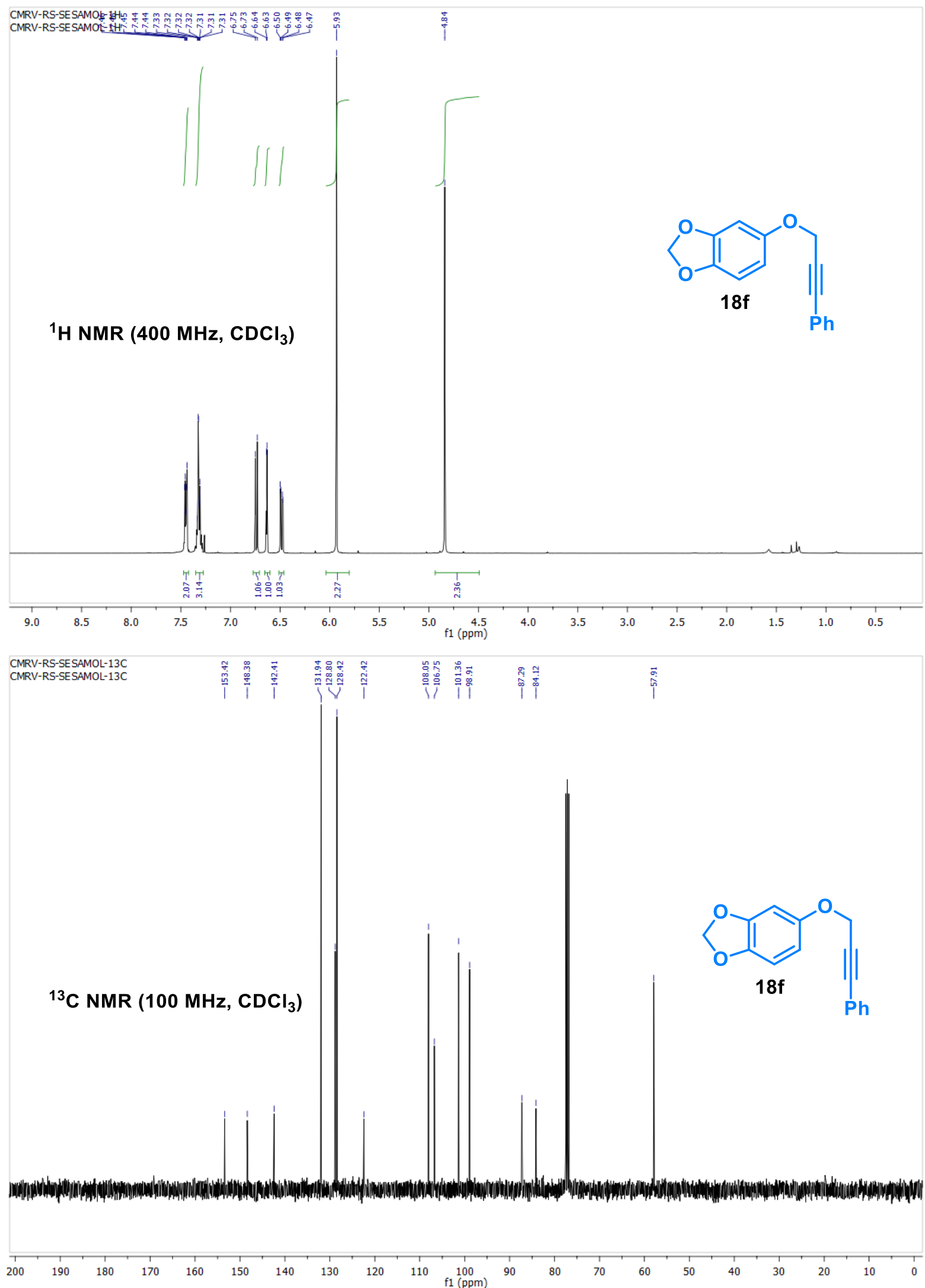

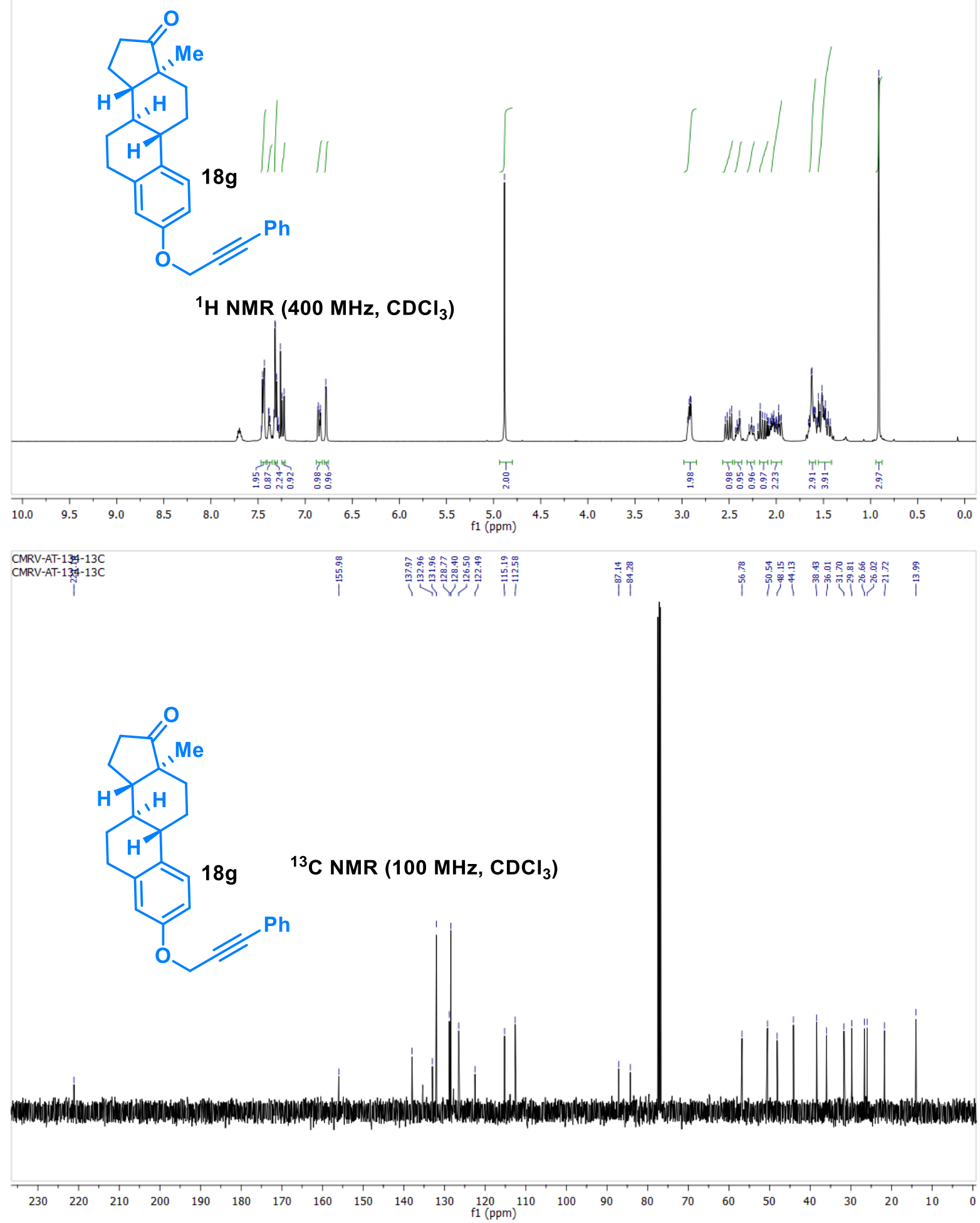

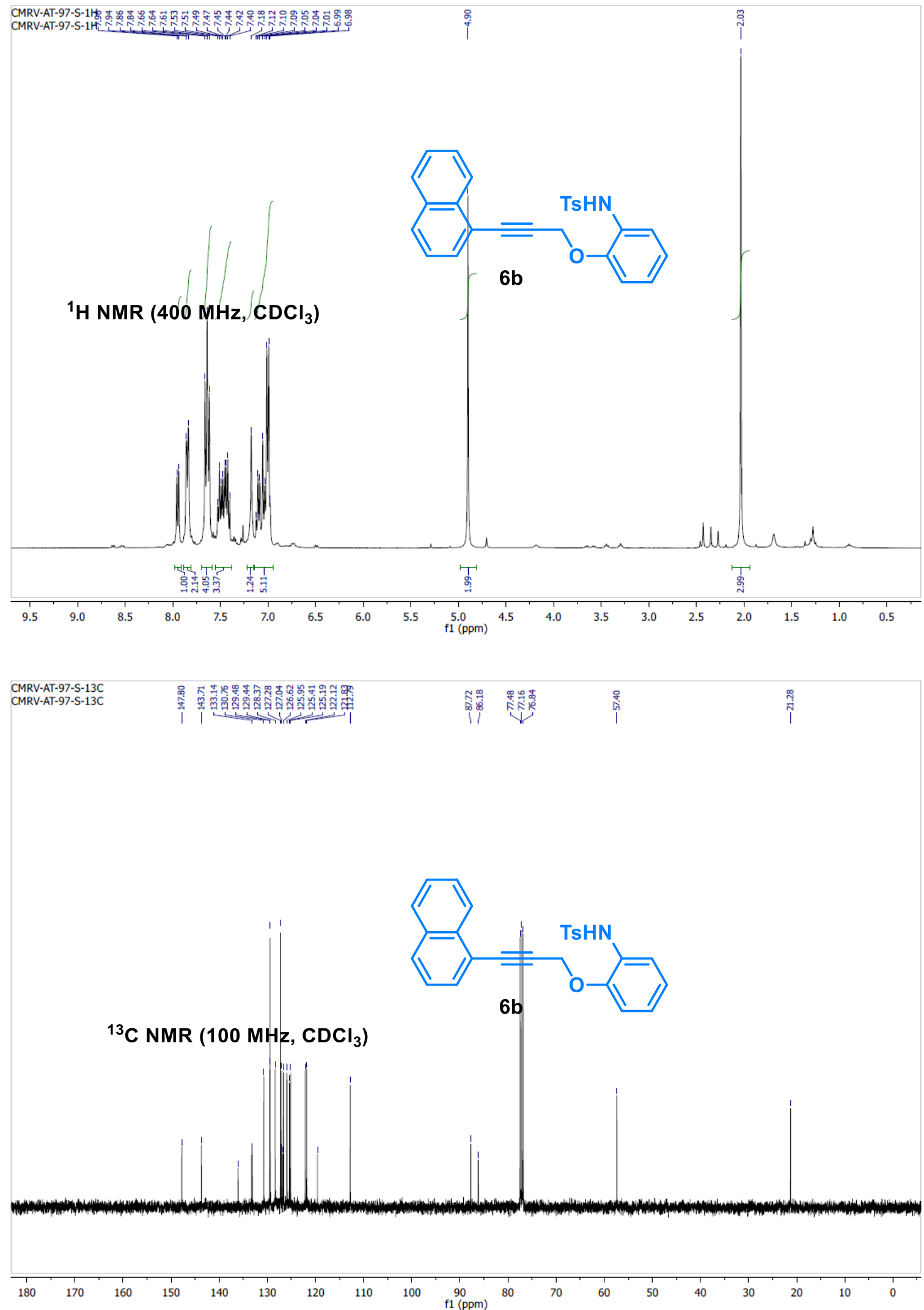

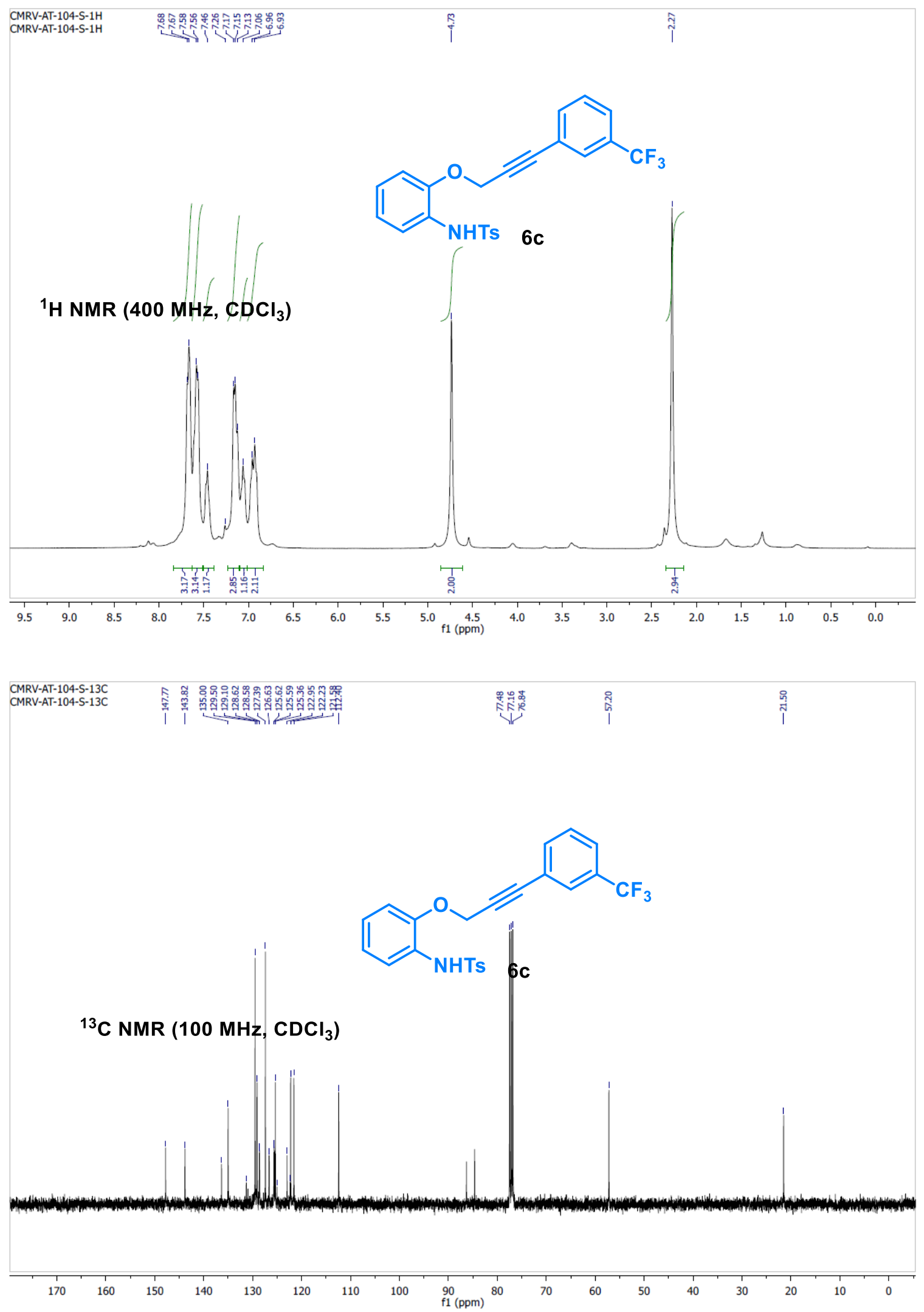

S68 

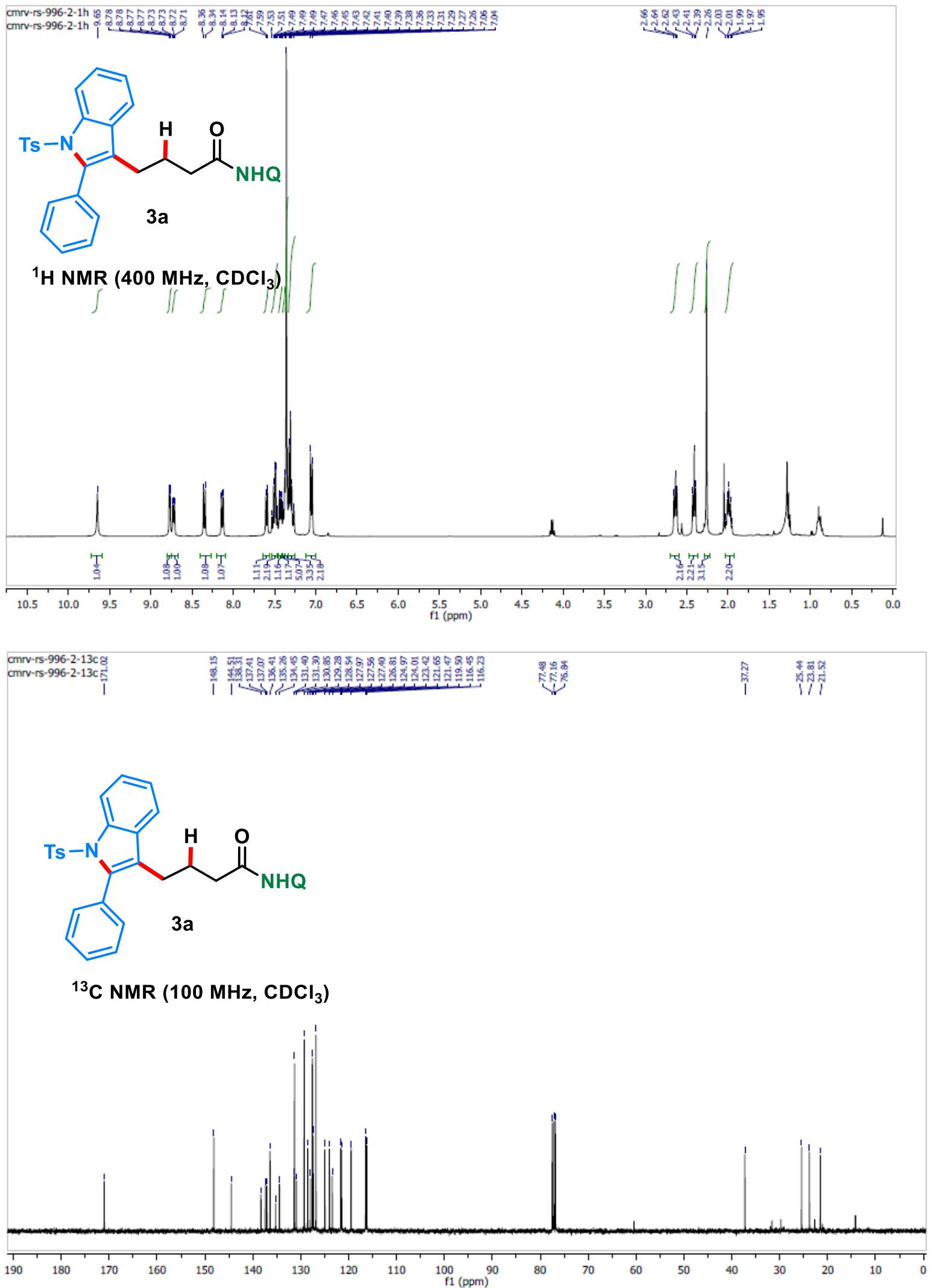

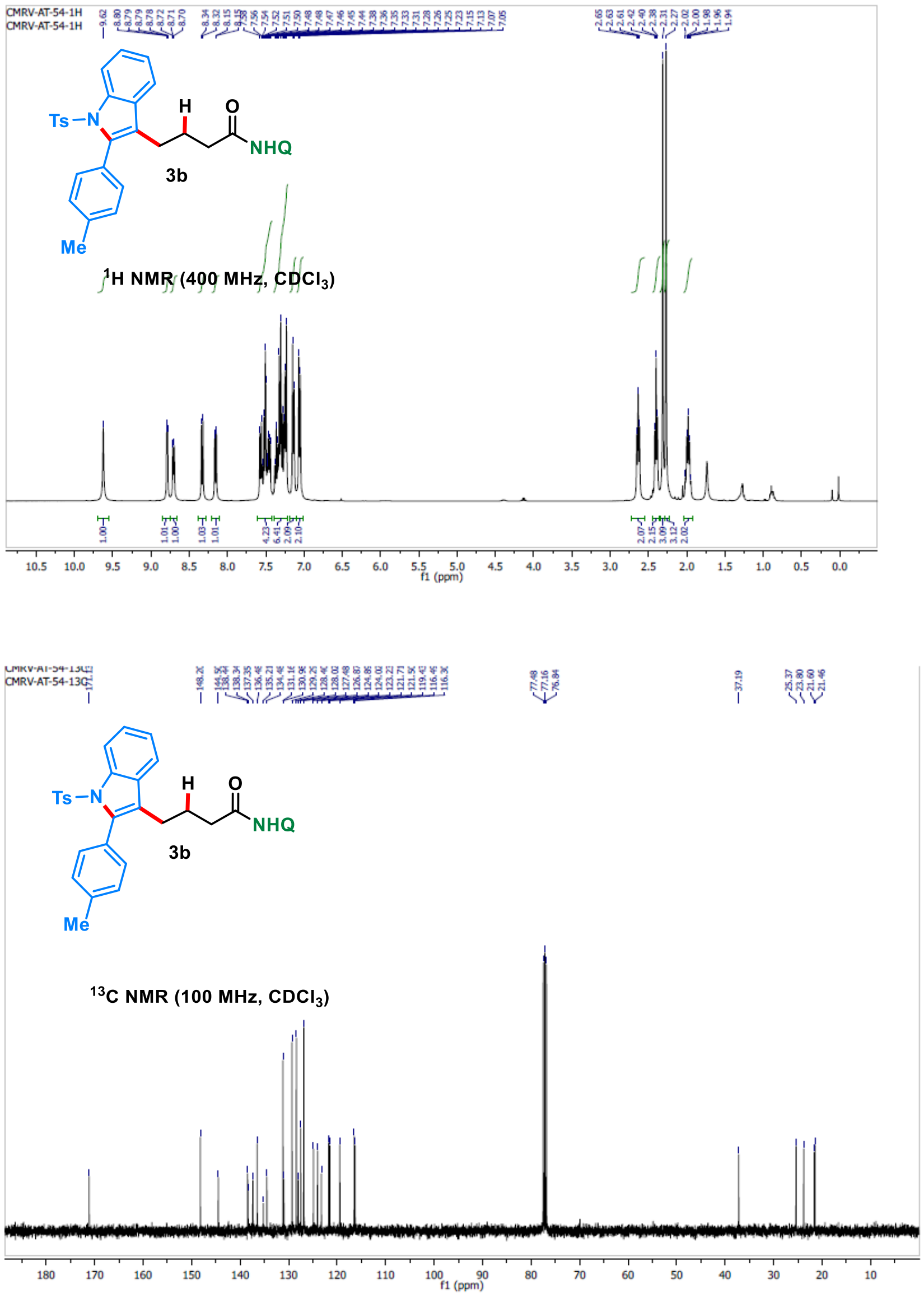

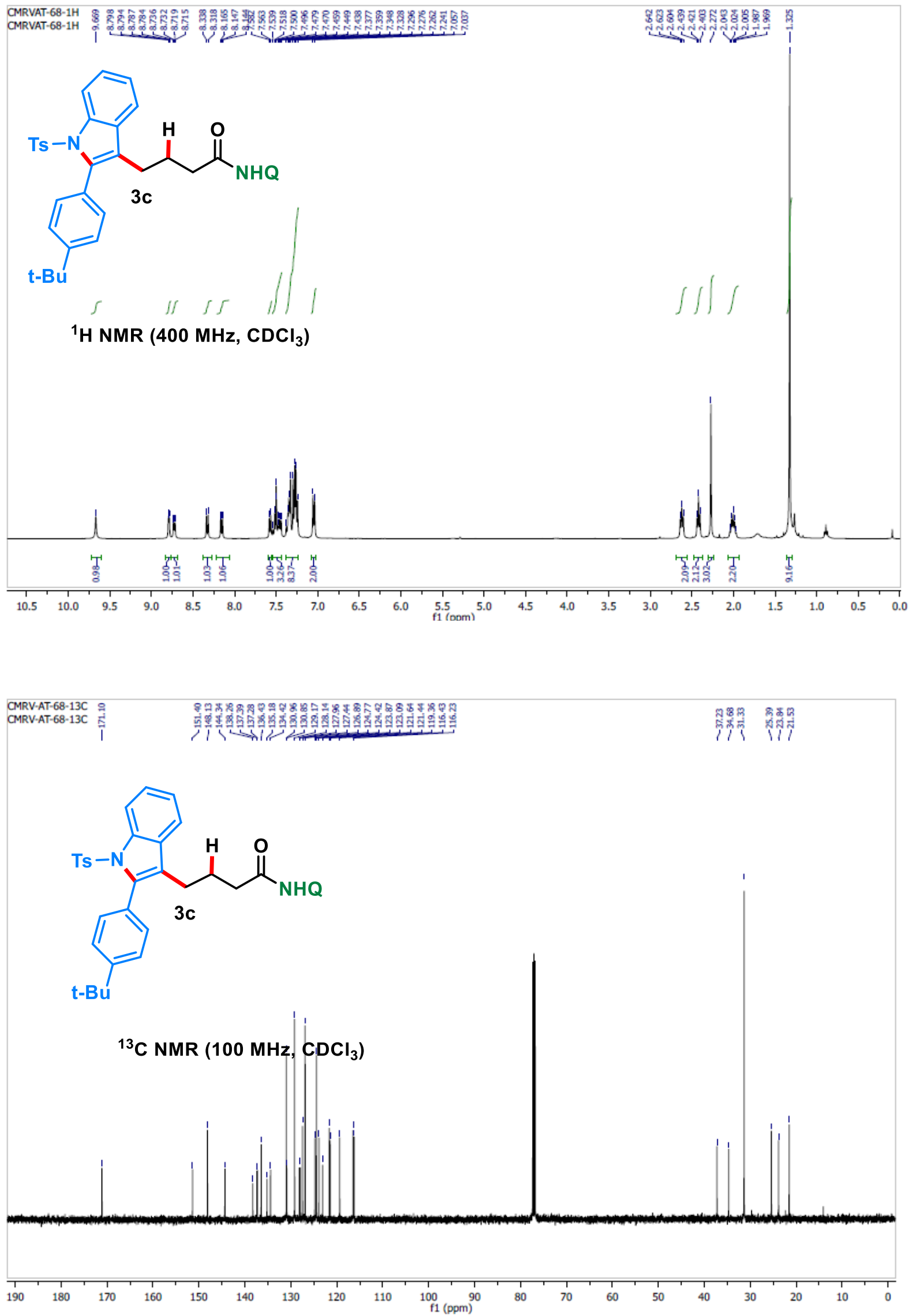

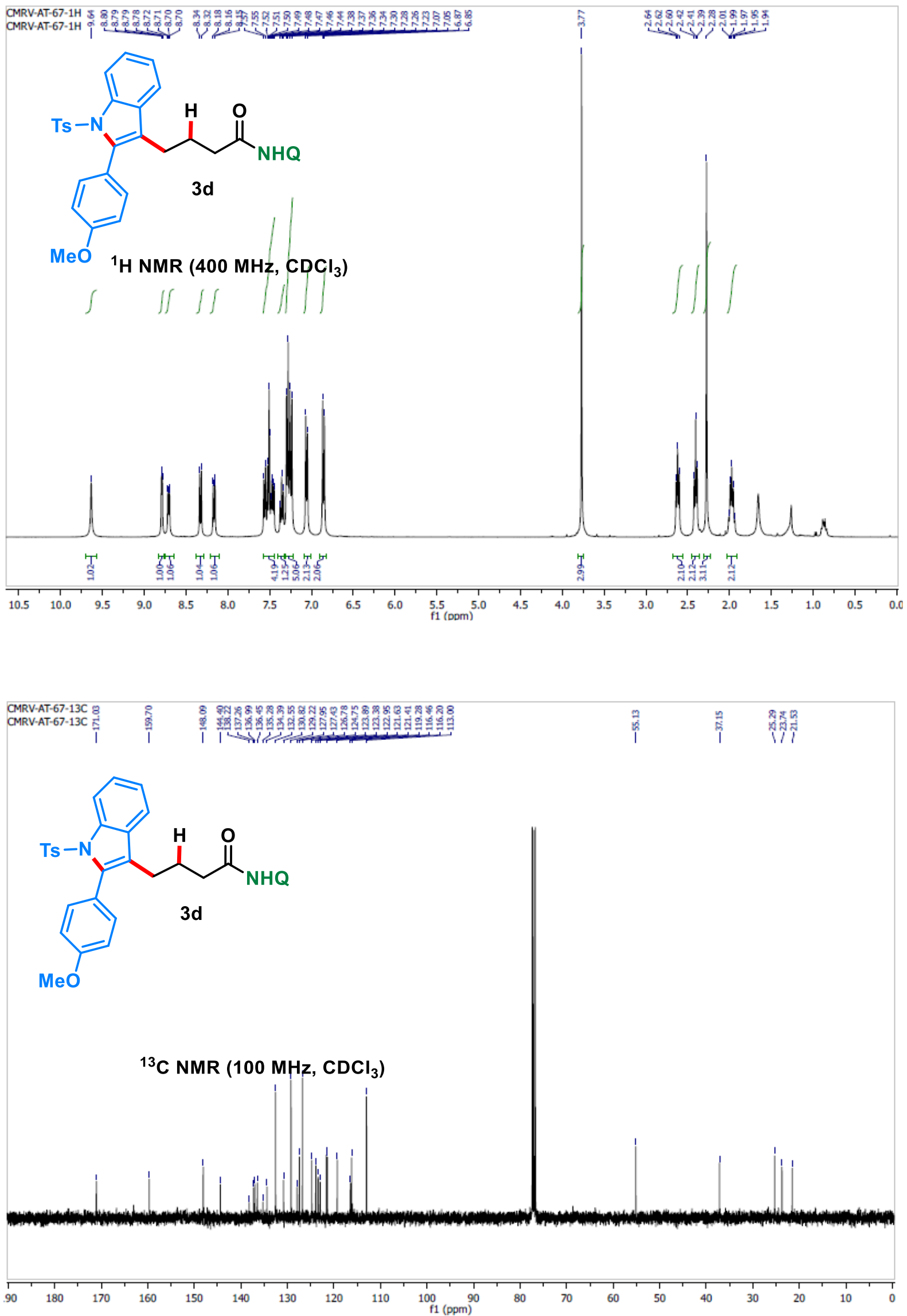

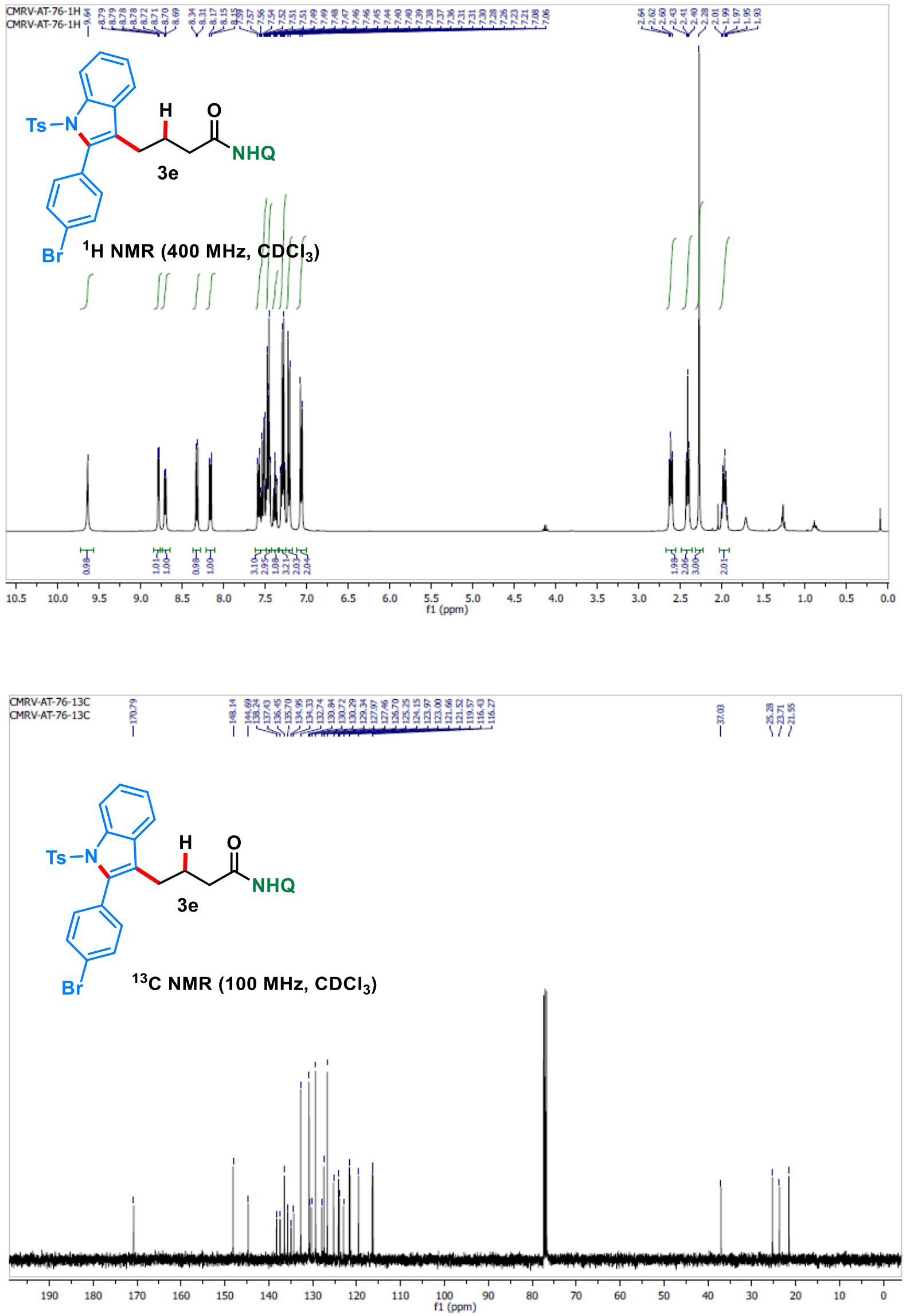

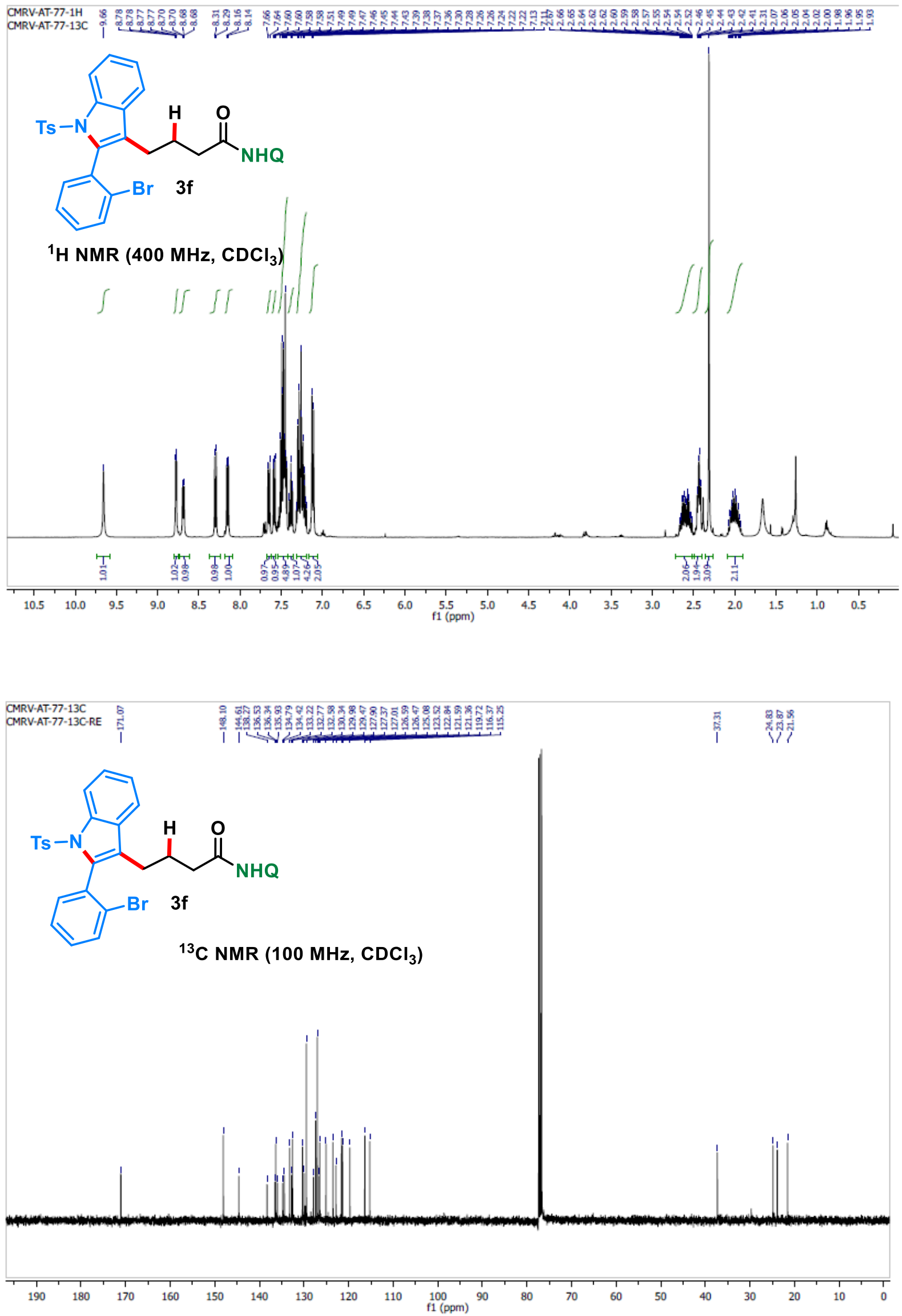

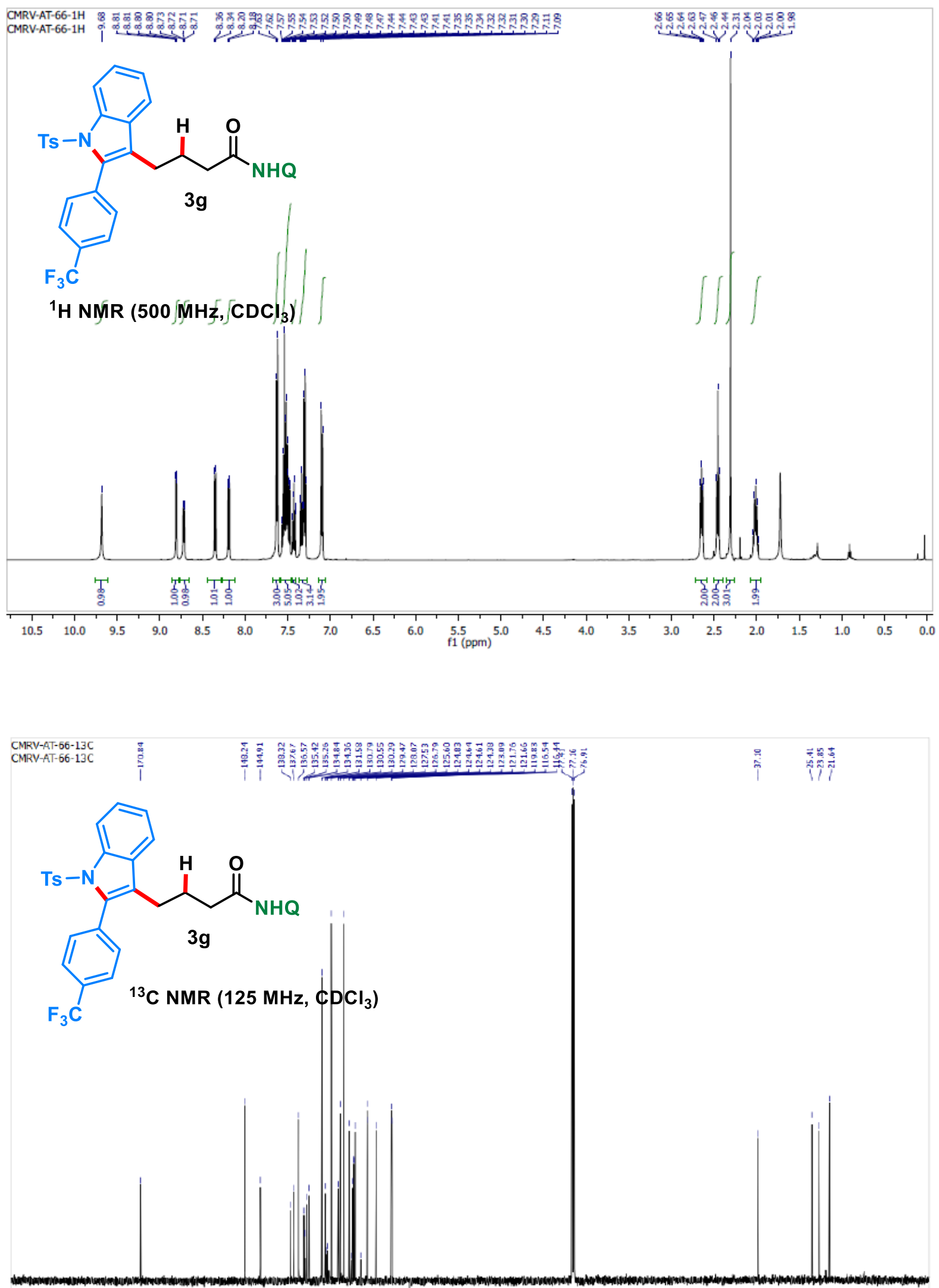

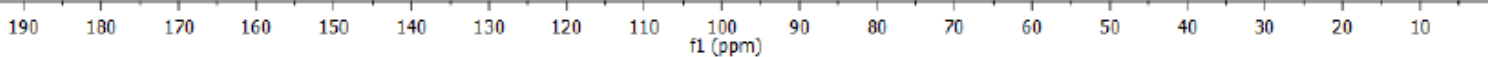



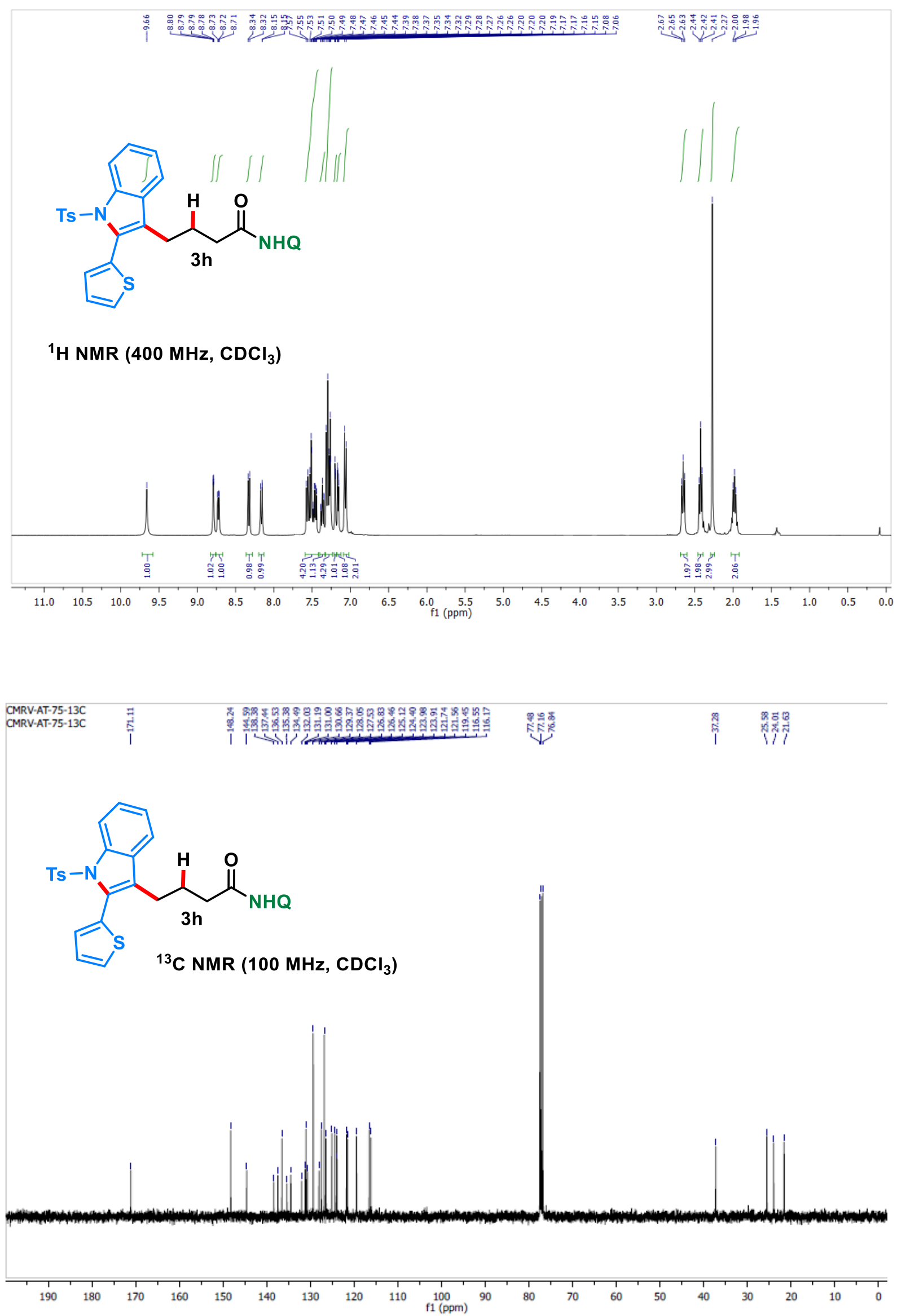

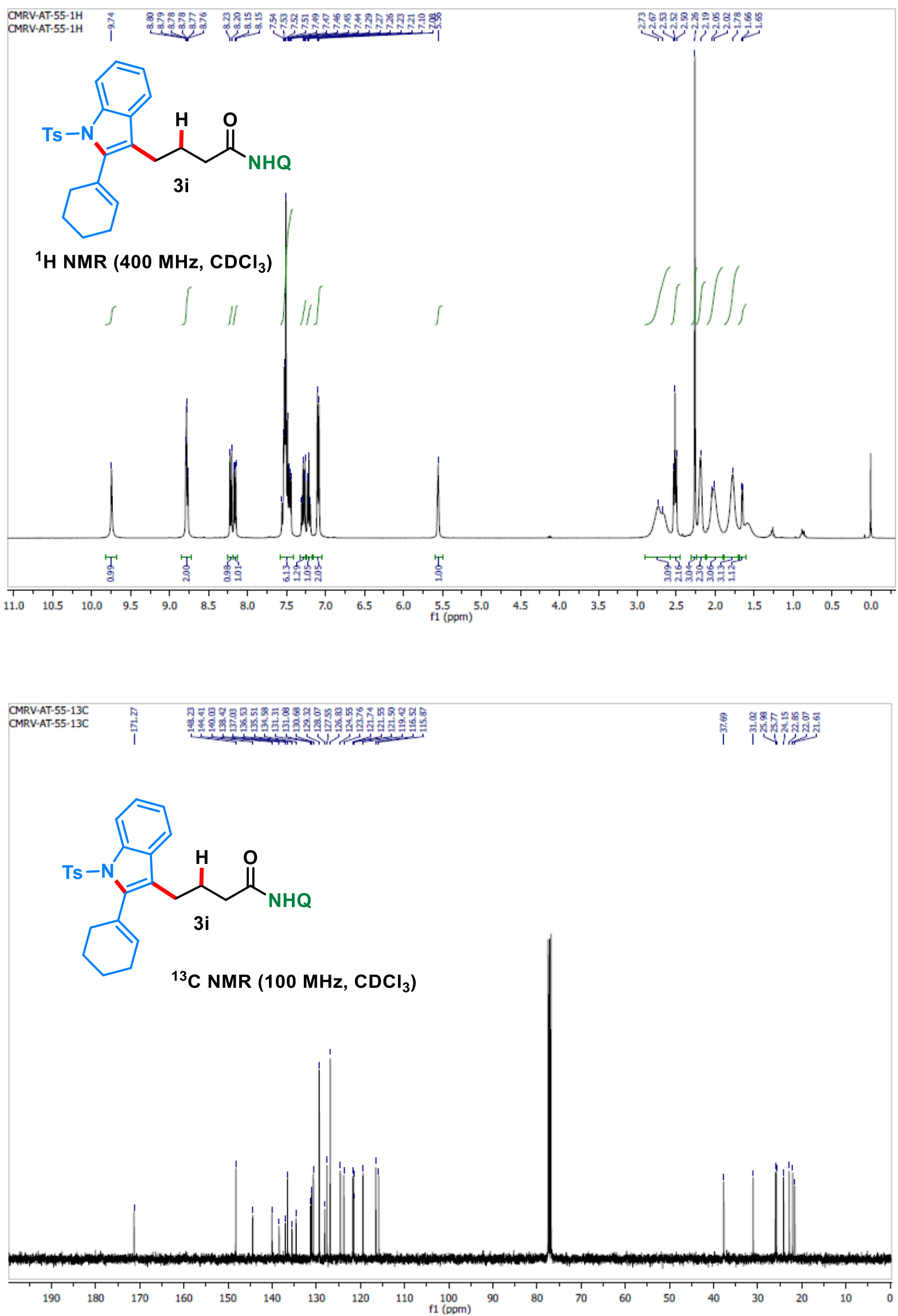

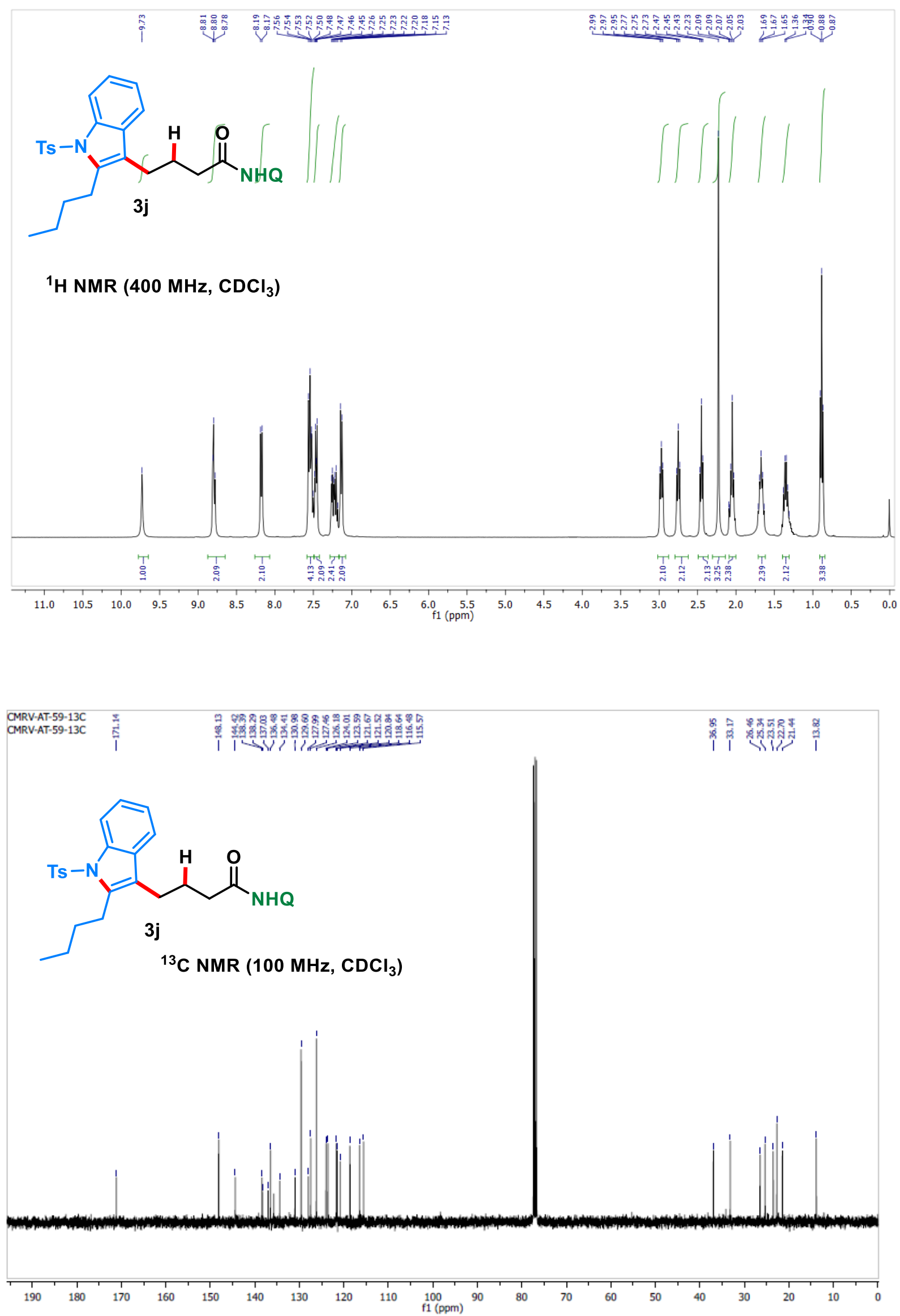

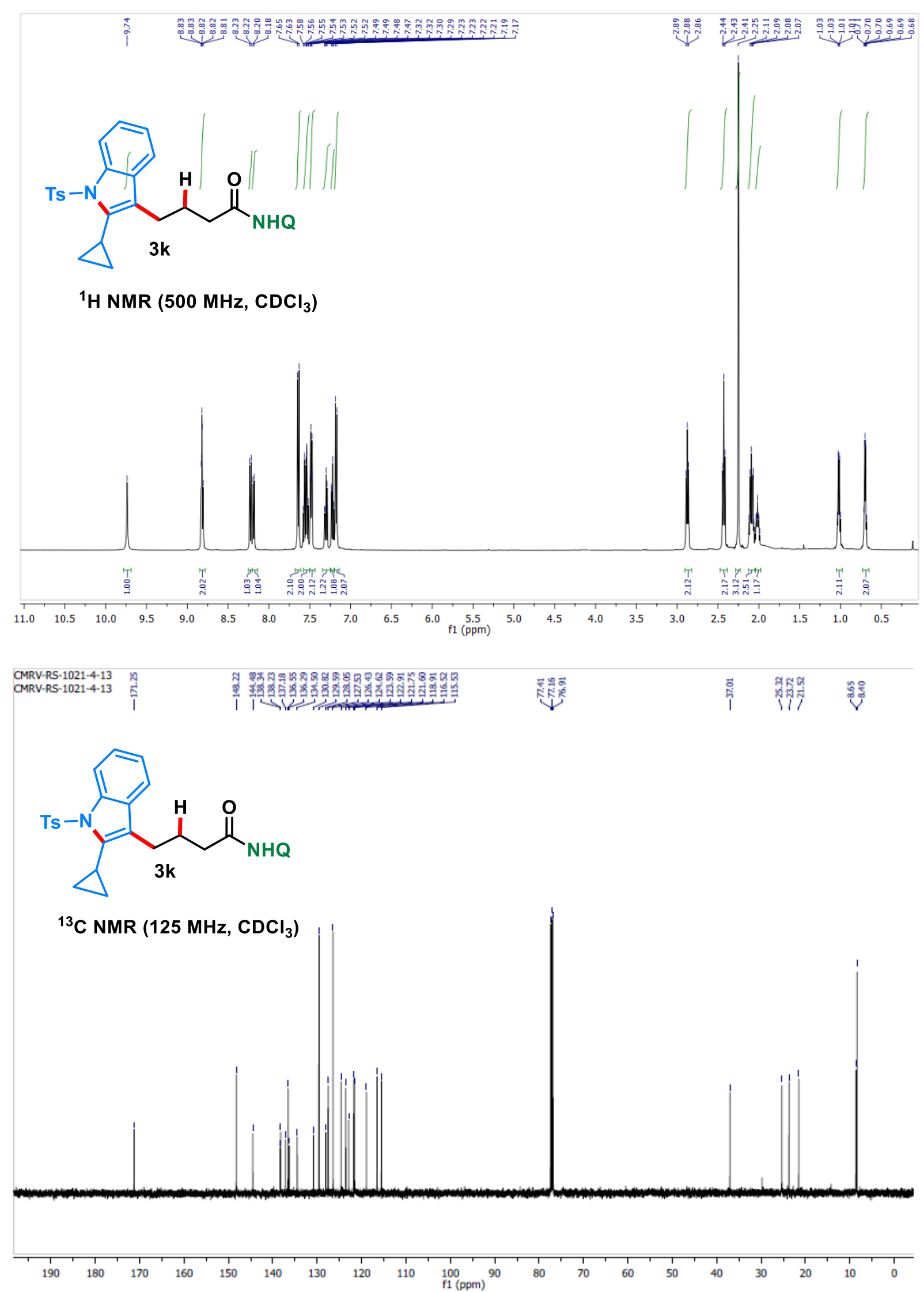

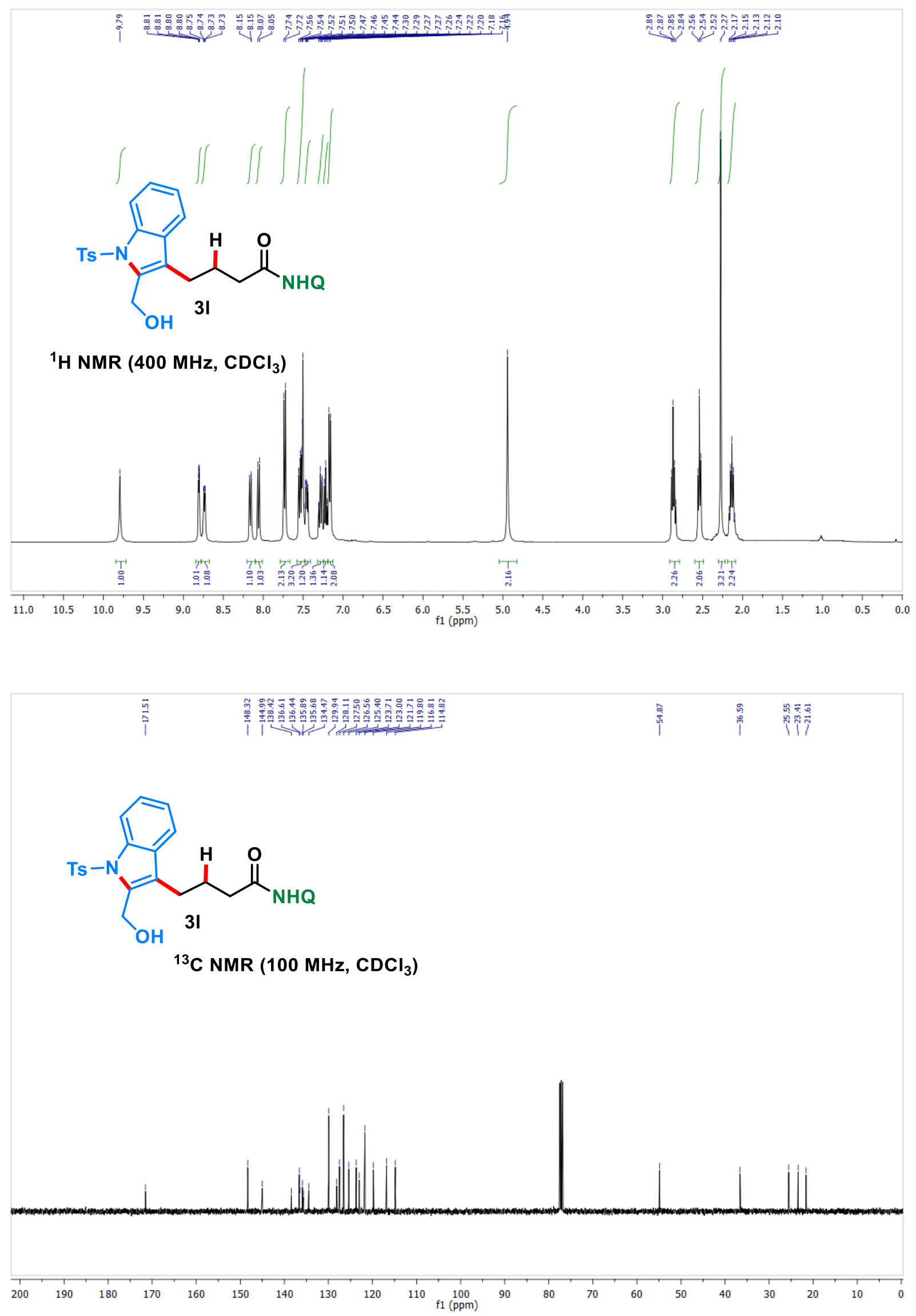

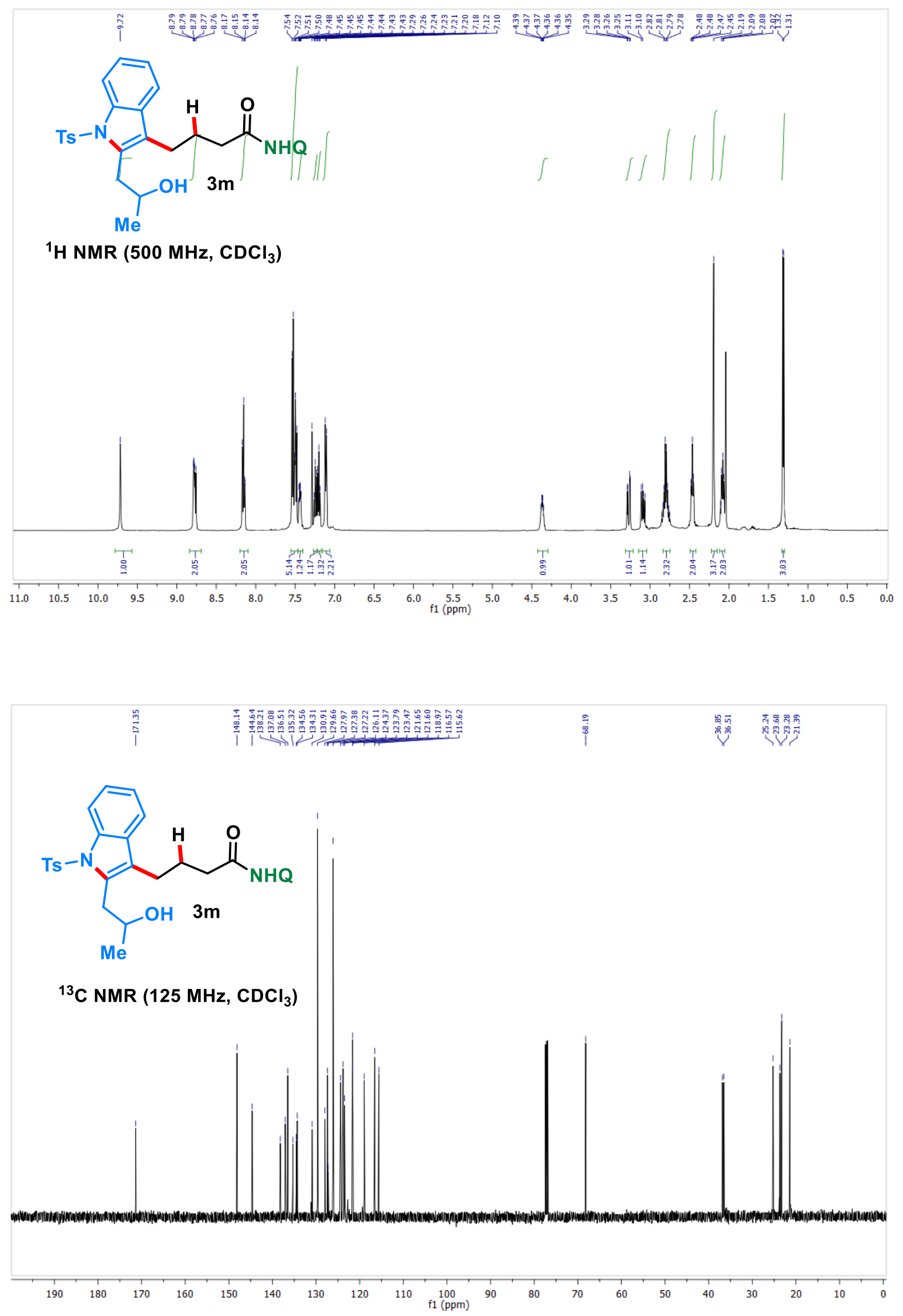

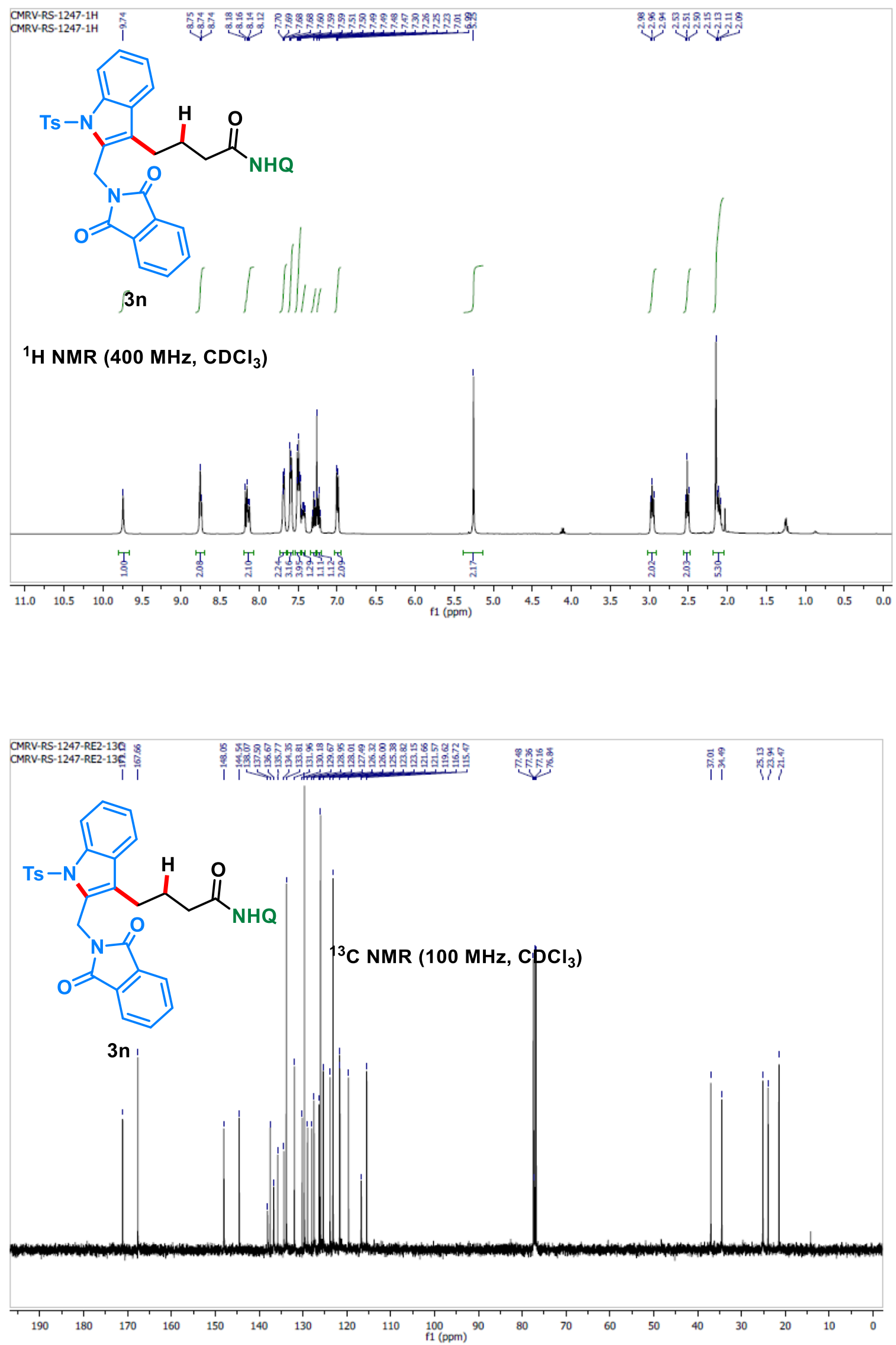

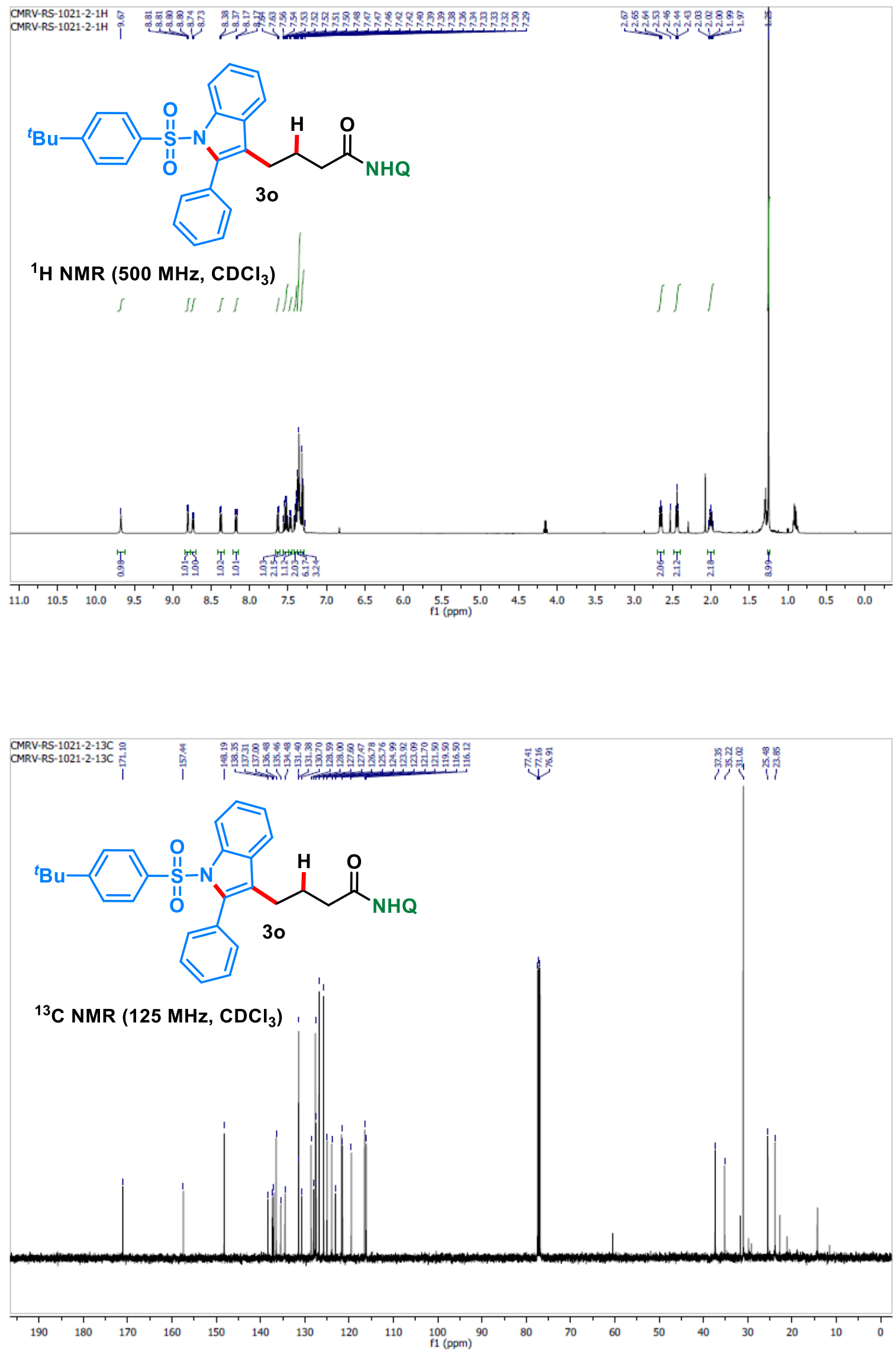


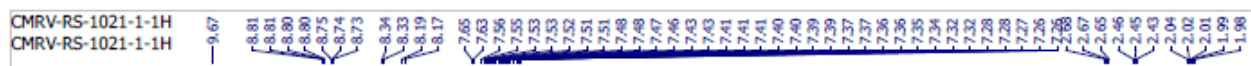
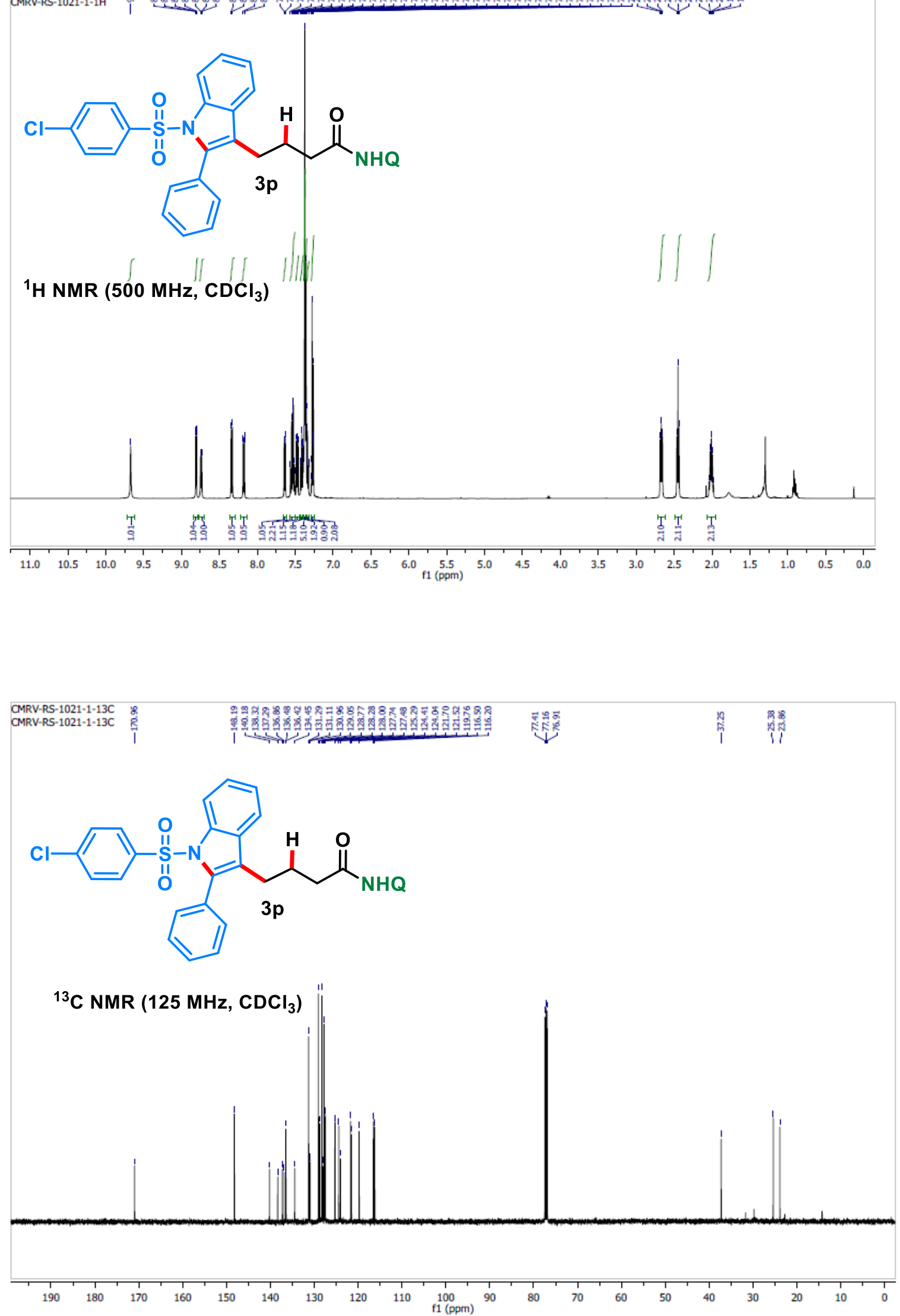


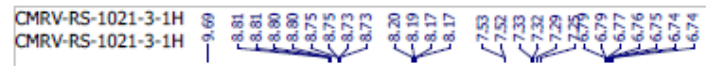

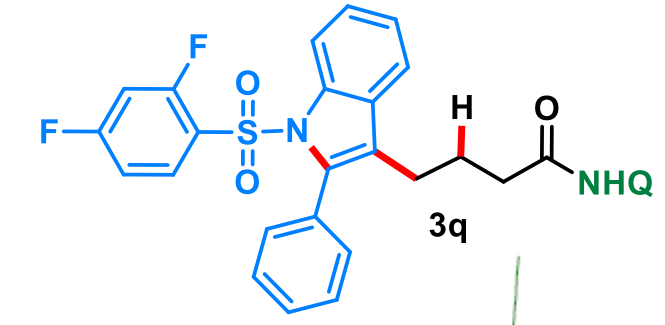

${ }^{1} \mathrm{H}$ NMR (500 MHz, $\left.\mathrm{CDCl}_{3}\right)$

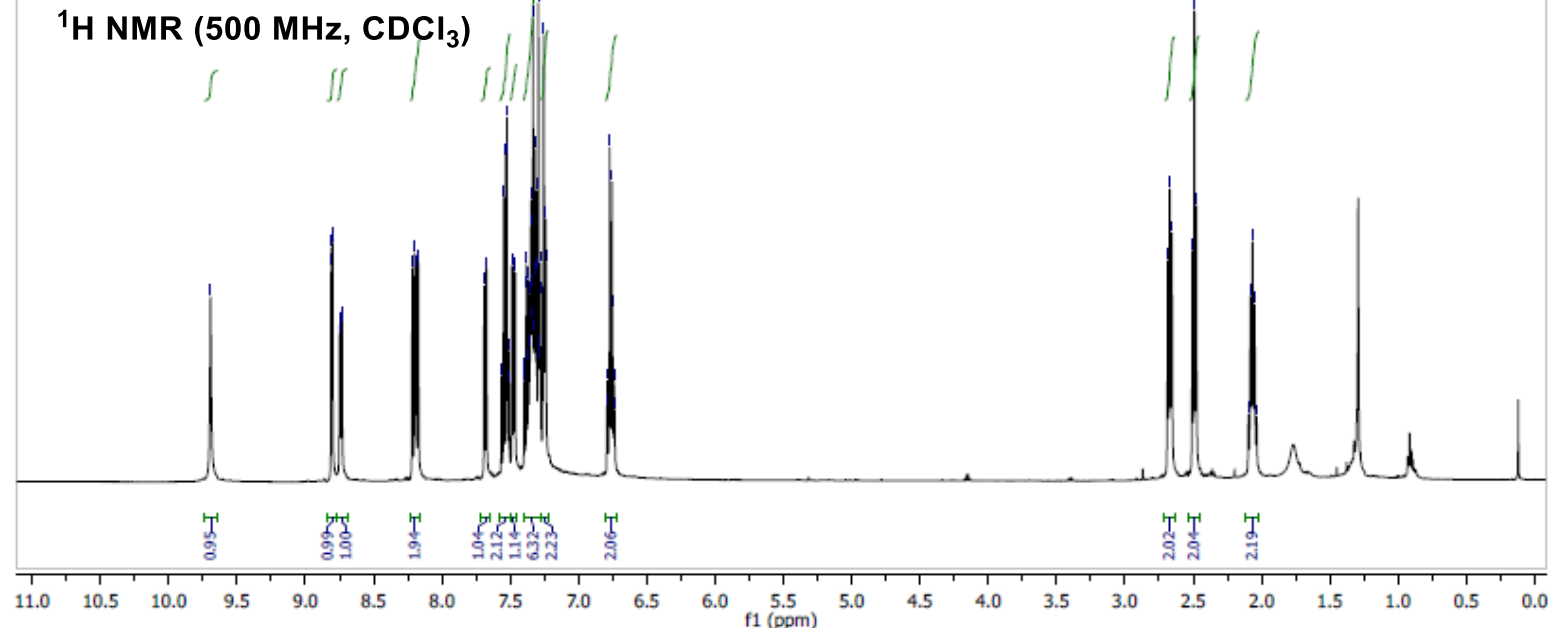

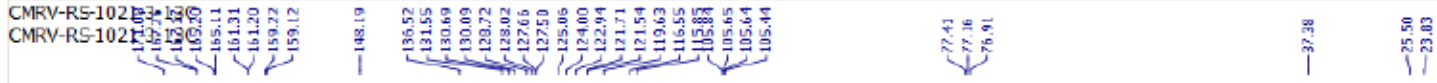<smiles>CC(CC(=O)NO)Cc1c(-c2ccccc2)n(S(=O)(=O)c2ccc(F)cc2F)c2ccccc12</smiles>

${ }^{13} \mathrm{C}$ NMR $\left(125 \mathrm{MHz}, \mathrm{CDCl}_{3}\right)$

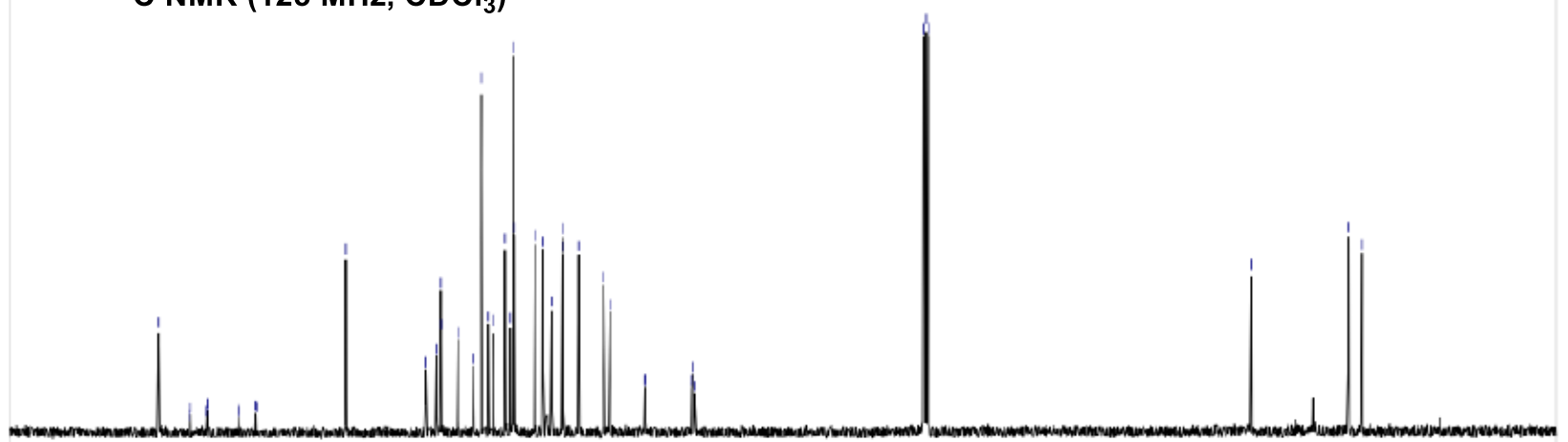

180

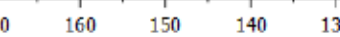

120

$110 \quad 100 \quad 90$

80 


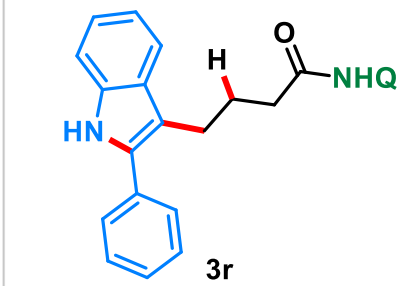

${ }^{1} \mathrm{H}$ NMR (400 MHz, $\left.\mathrm{CDCl}_{3}\right)$
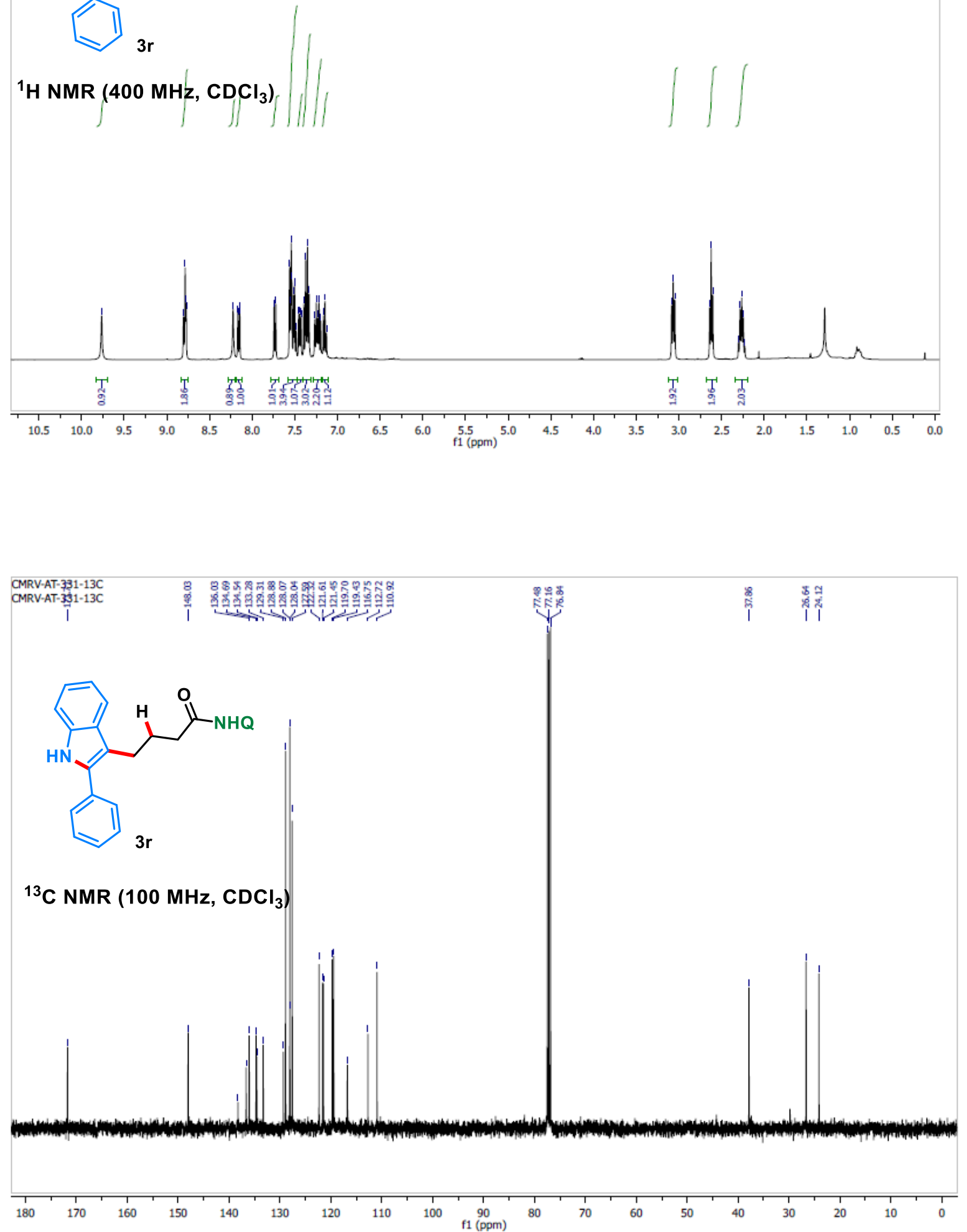
<smiles>[Y9]C(CC(=O)NO)c1c(C2=CC=CC2)n([13CH3])c2ccccc12</smiles>

3s, $86 \%$

${ }^{1} \mathrm{H}$ NMR $\left.(400 / \mathrm{MHz}, C \mathrm{CDCl})_{3}\right) \int \Downarrow /$

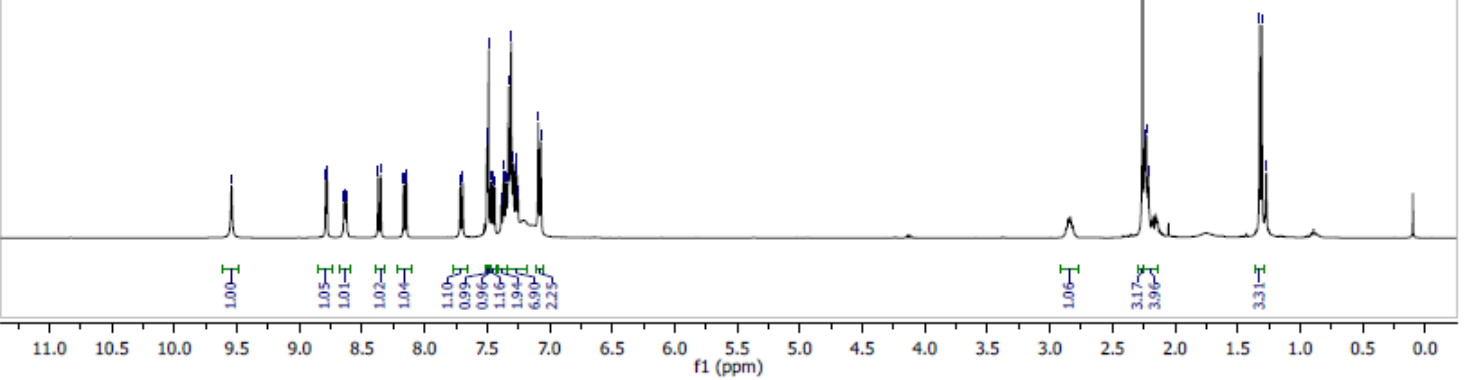

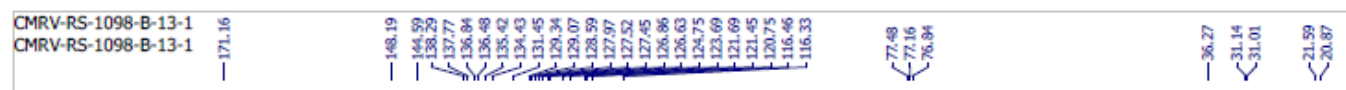

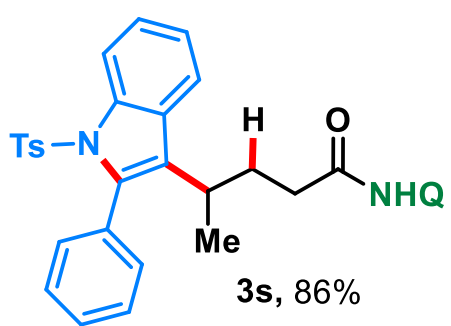

${ }^{13} \mathrm{C}$ NMR $\left(100 \mathrm{MHz}, \mathrm{CDCl}_{3}\right)$
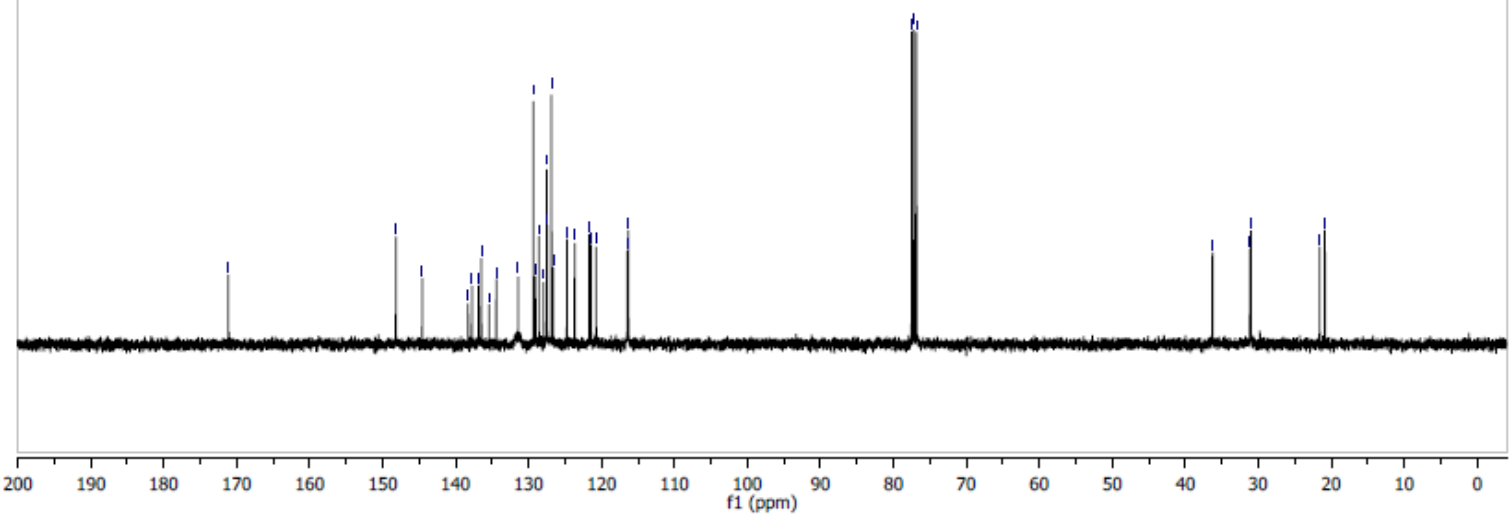


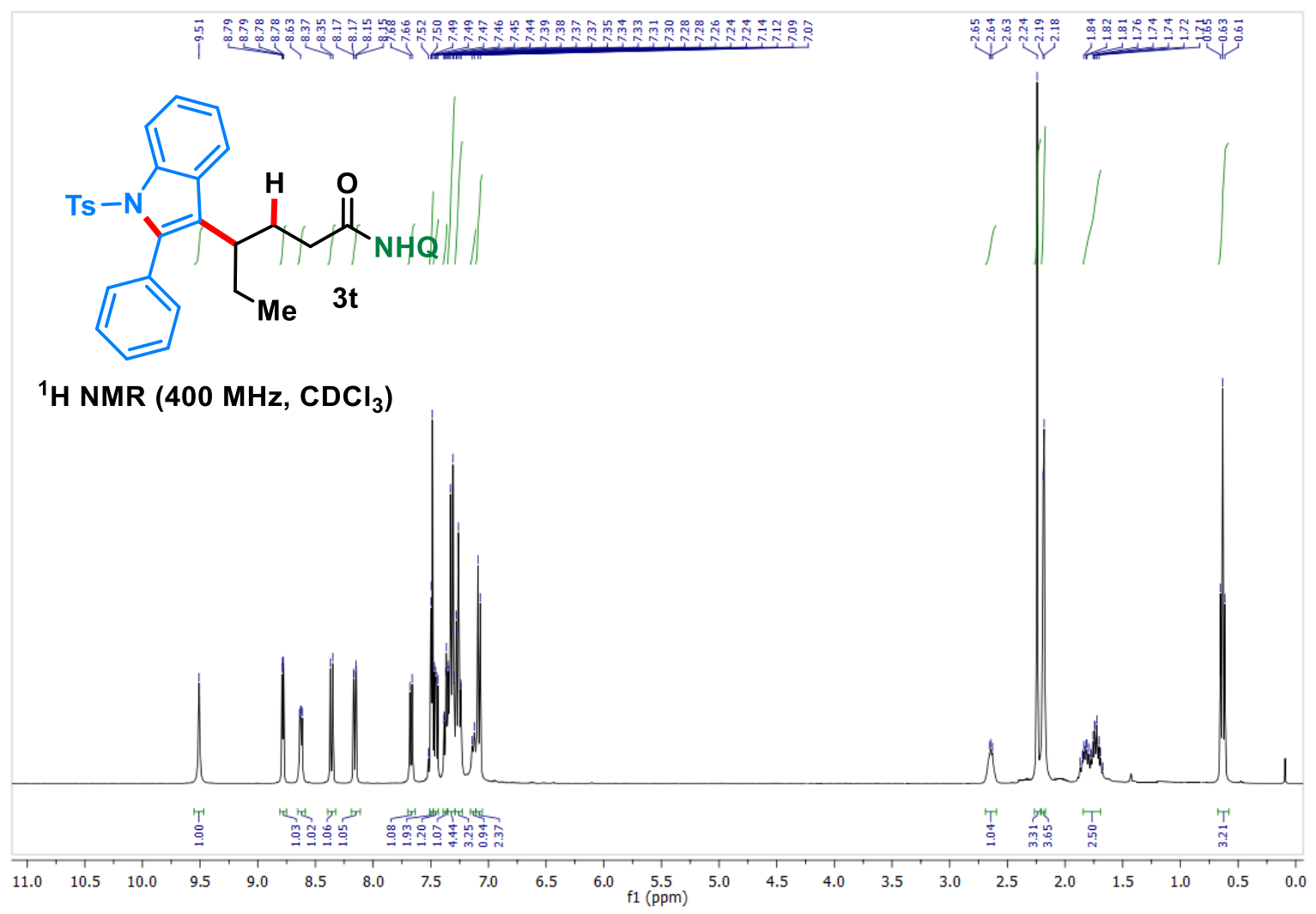

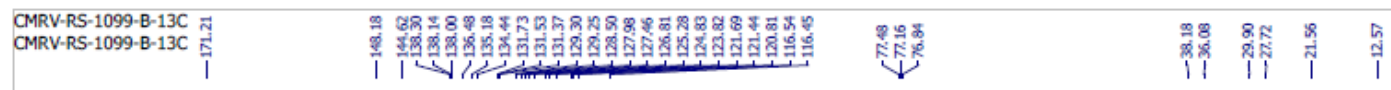<smiles>CCC(CC(=O)NO)C(CC)c1c(-c2ccccc2)n([125I])c2ccccc12</smiles>

${ }^{13} \mathrm{C} \mathrm{NMR}\left(100 \mathrm{MHz}, \mathrm{CDCl}_{3}\right)$

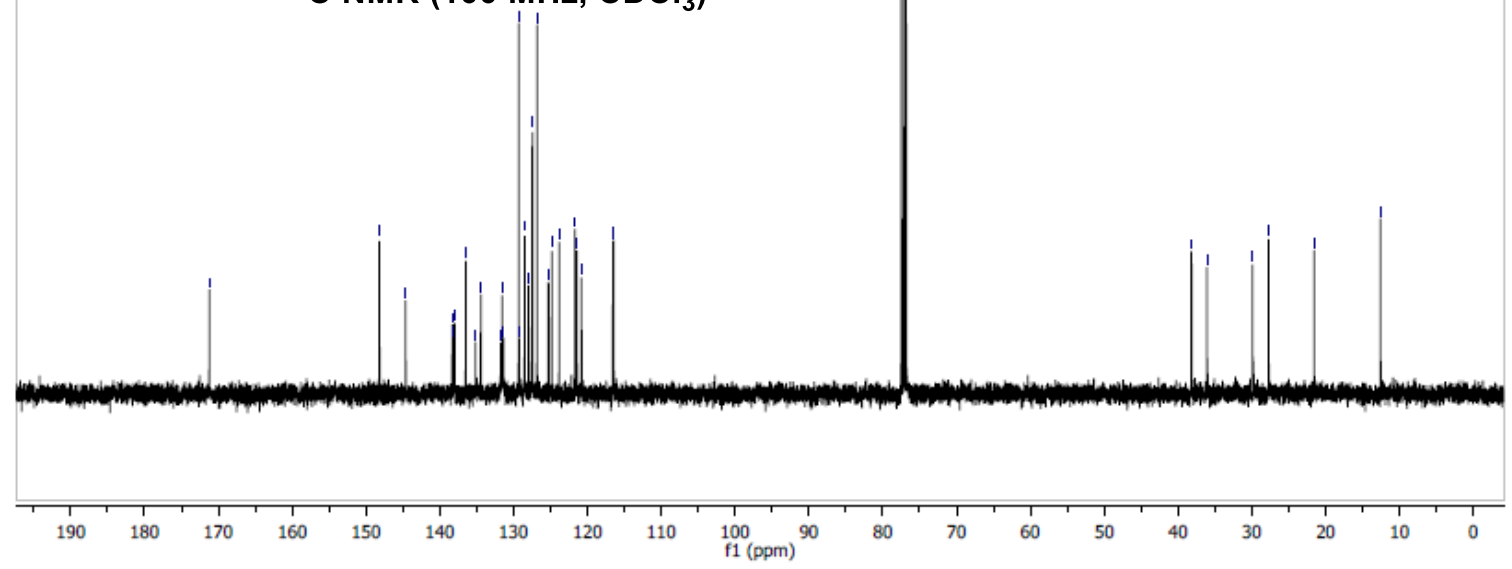




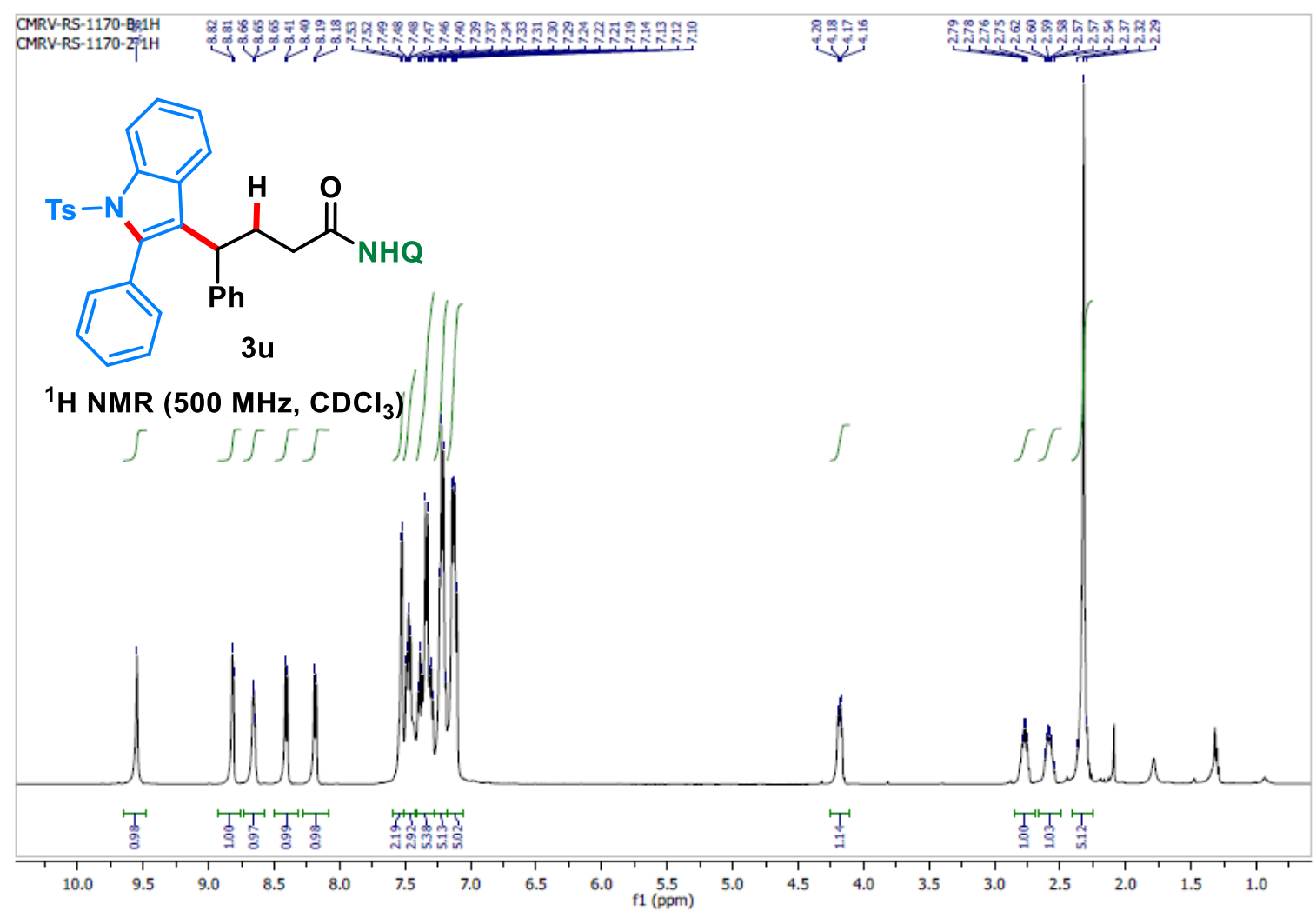

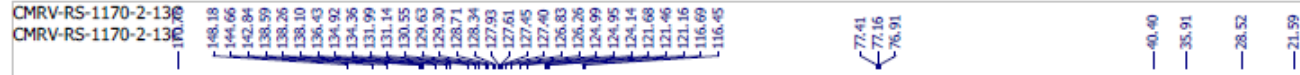<smiles>O=C(CC(c1ccccc1)c1c(-c2ccccc2)n([135I])c2ccccc12)NO</smiles>

${ }^{13} \mathrm{C}$ NMR (125 MHz, $\mathrm{CDCl}_{3}$ )
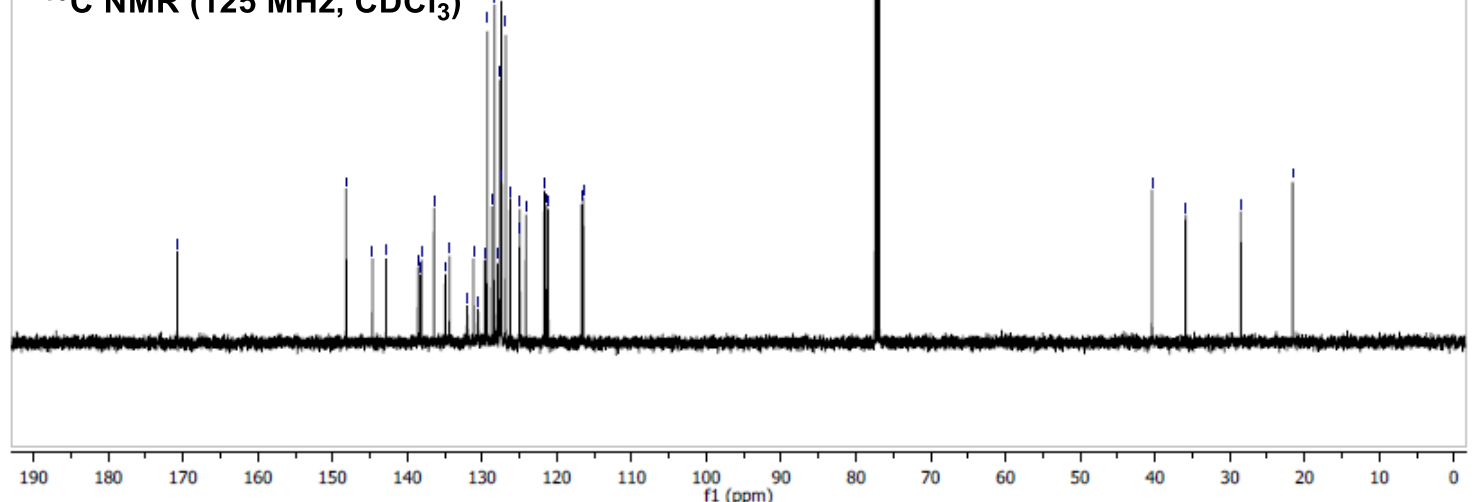

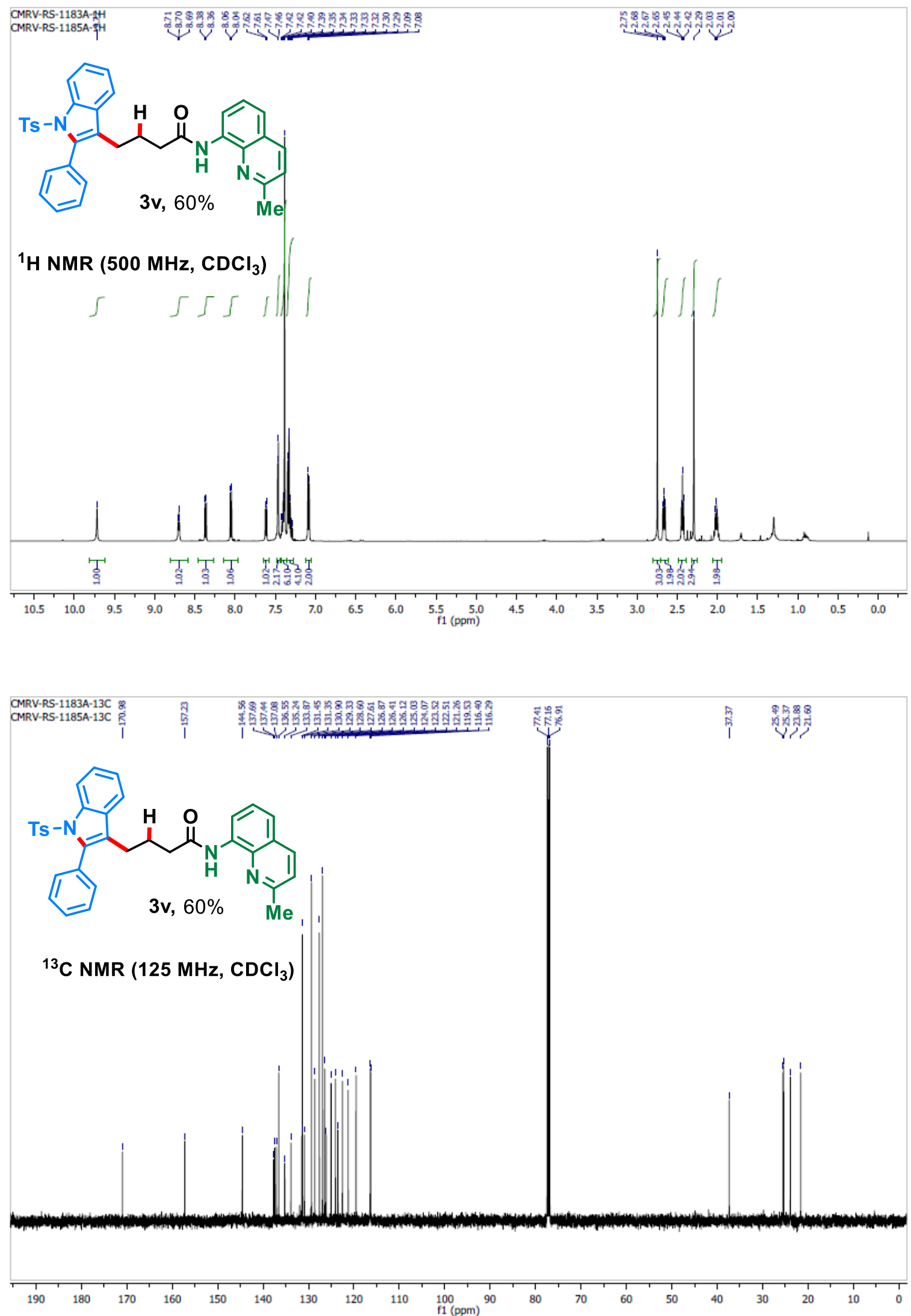


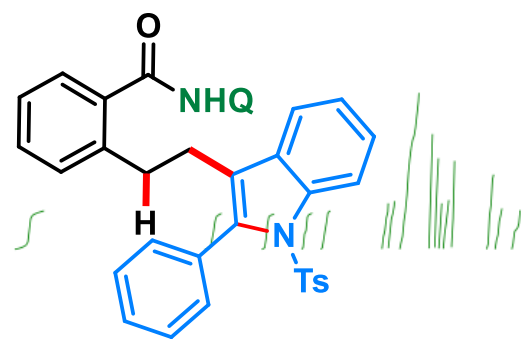

$3 w, 30 \%$

${ }^{1} \mathrm{H}$ NMR $\left(400 \mathrm{MHz}, \mathrm{CDCl}_{3}\right)$

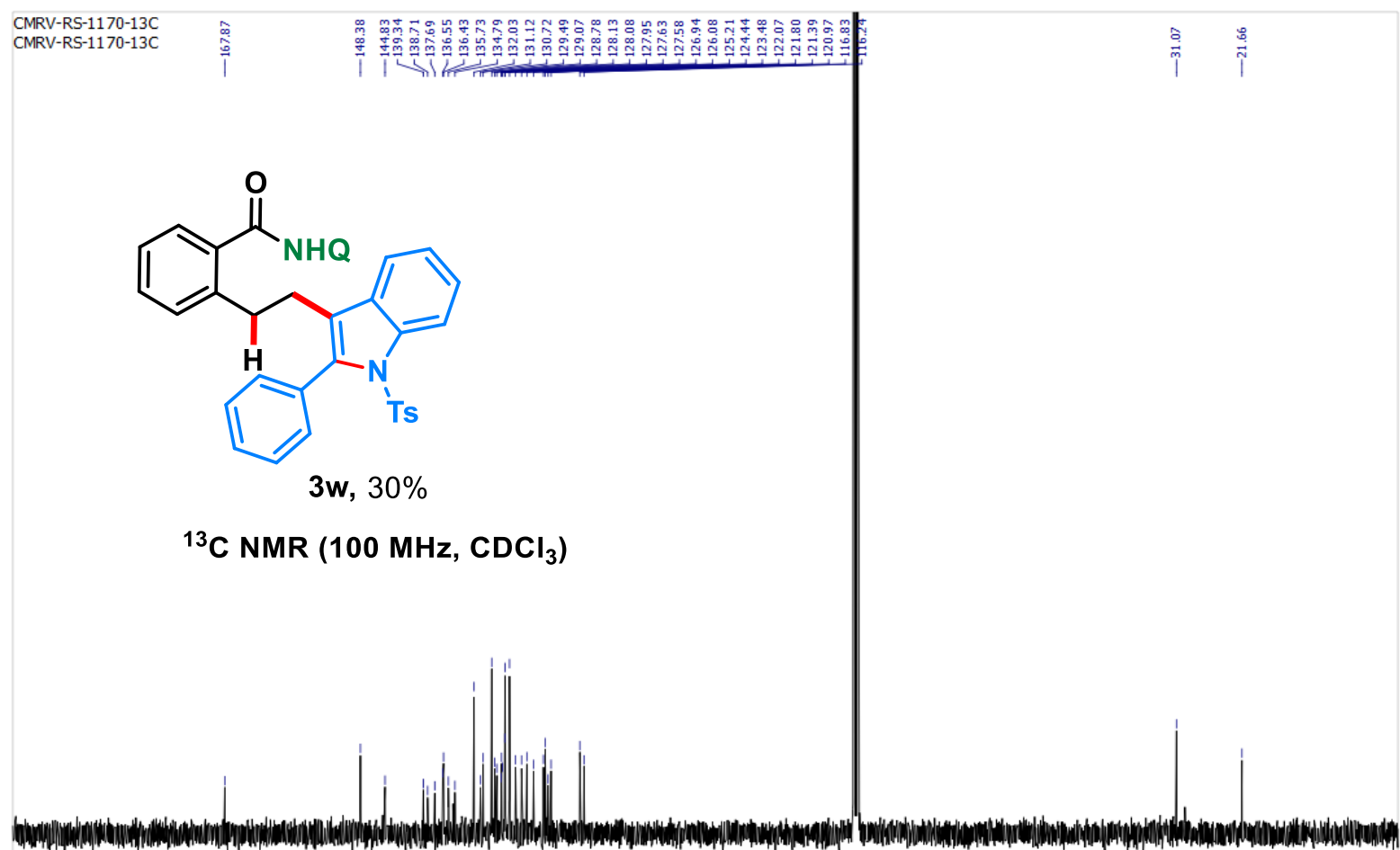

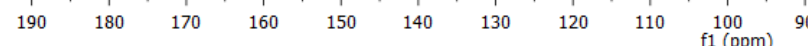




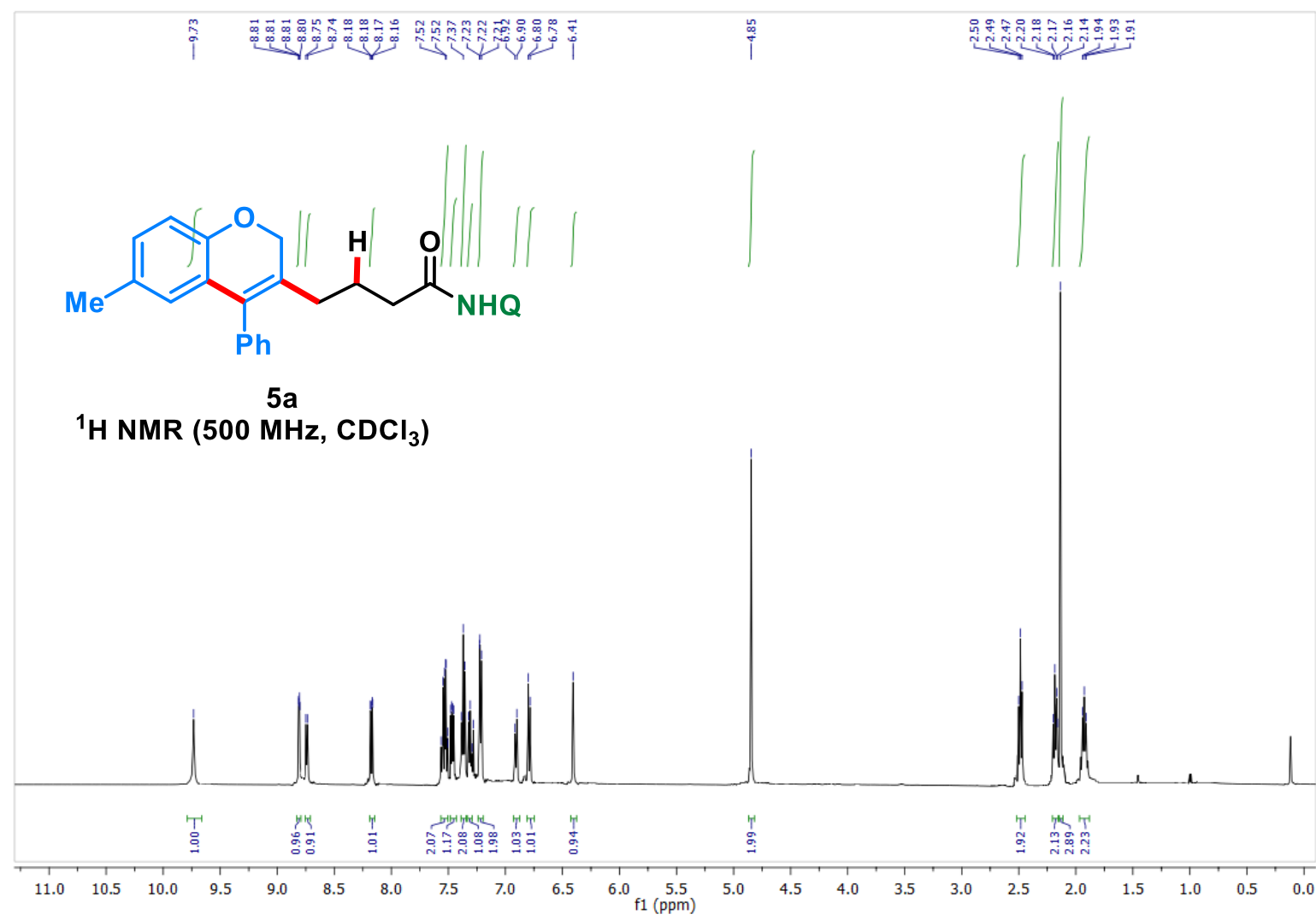

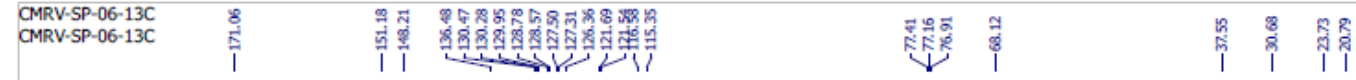<smiles>Cc1ccc2c(c1)C(c1ccccc1)=C(CCC(=O)NO)CO2</smiles>

$5 a$
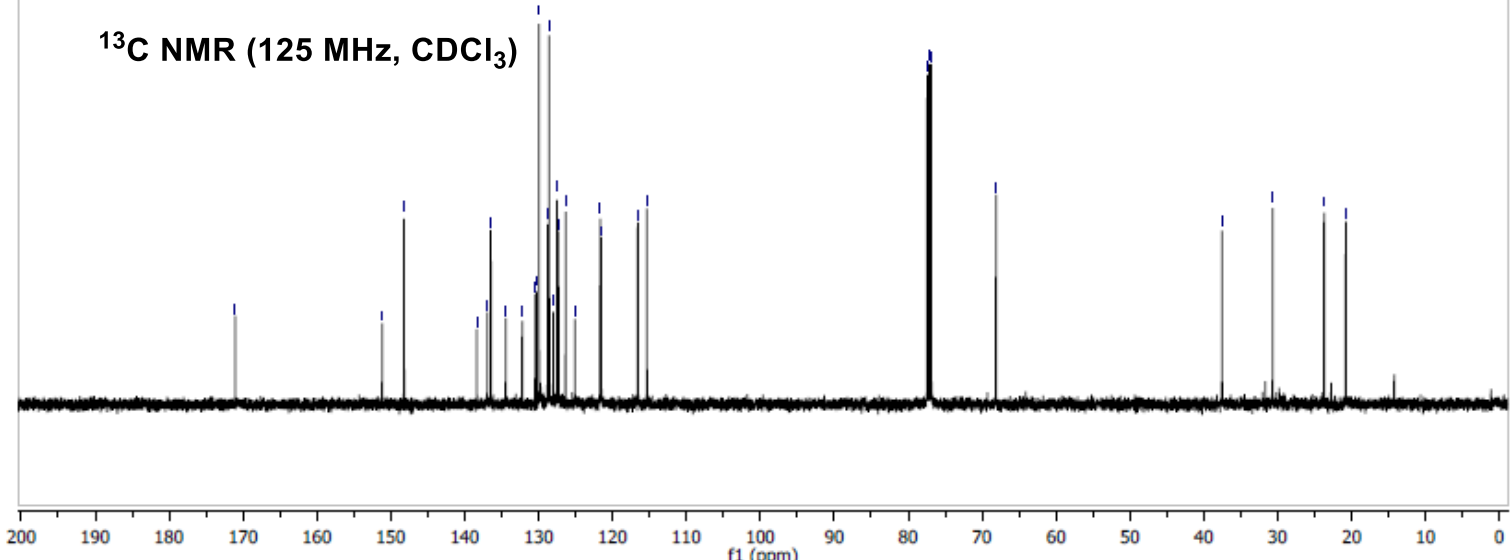
CMRV-RS-1264-1H
CMRV-RS-1264-1H<smiles>Cc1ccc2c(c1)C(c1ccccc1C)=C(CCC(=O)NO)CO2</smiles>

$5 b$

${ }^{1} \mathrm{H}$ NMR $\left(400 \mathrm{MHz}, \mathrm{CDCl}_{3}\right)$
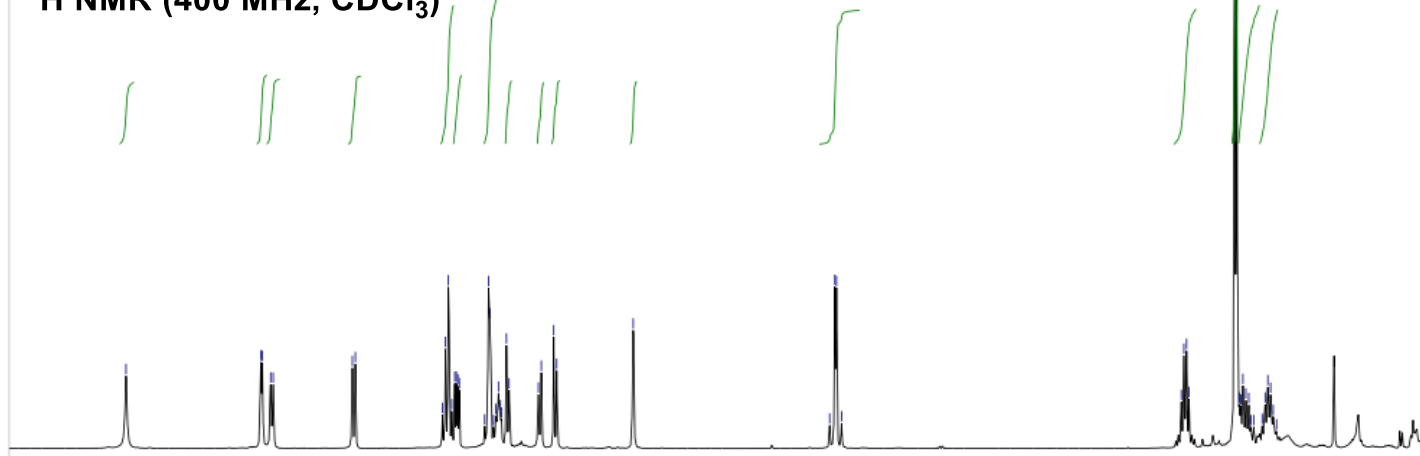

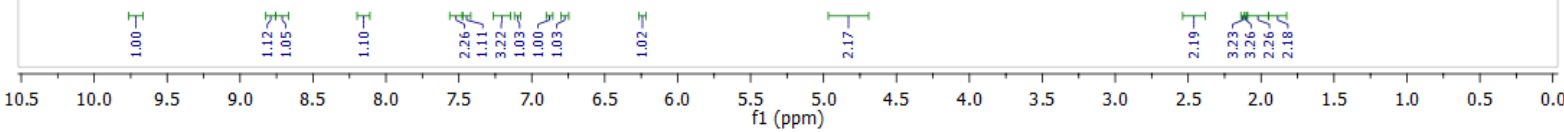

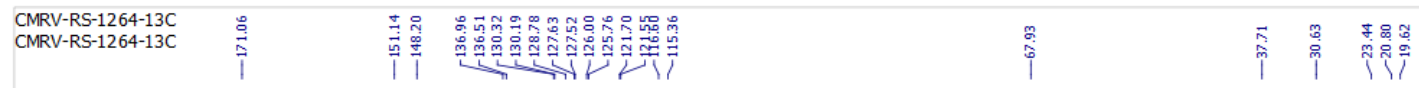<smiles>Cc1ccc2c(c1)C(c1ccccc1C)=C(CCC(=O)NO)CO2</smiles>

${ }^{13} \mathrm{C}$ NMR $\left(100 \mathrm{MHz}, \mathrm{CDCl}_{3}\right)$

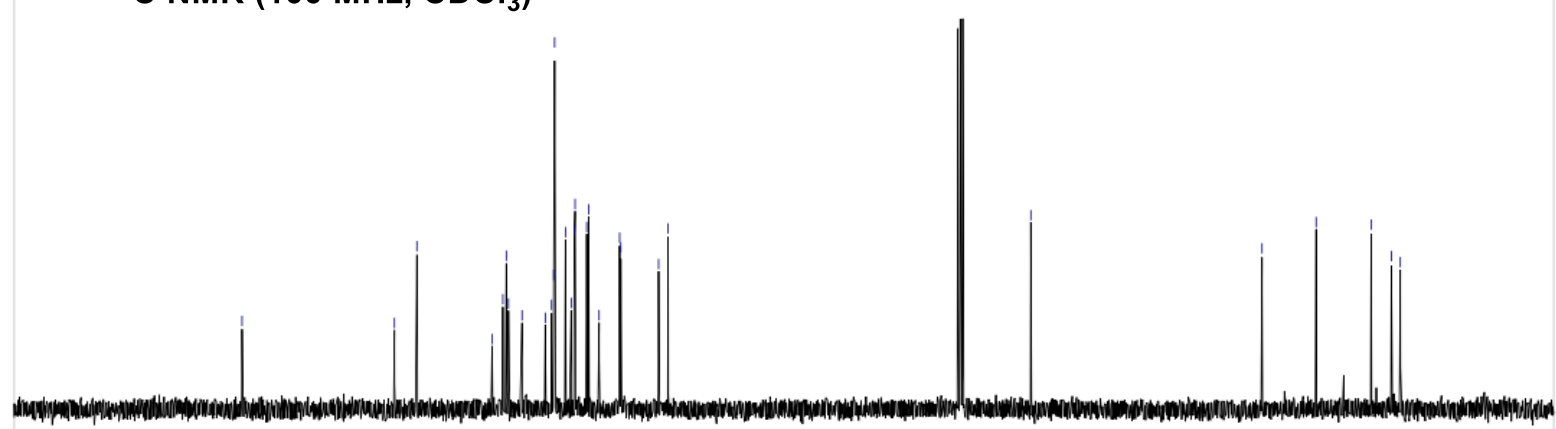

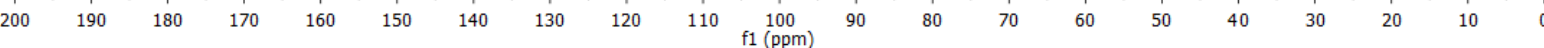



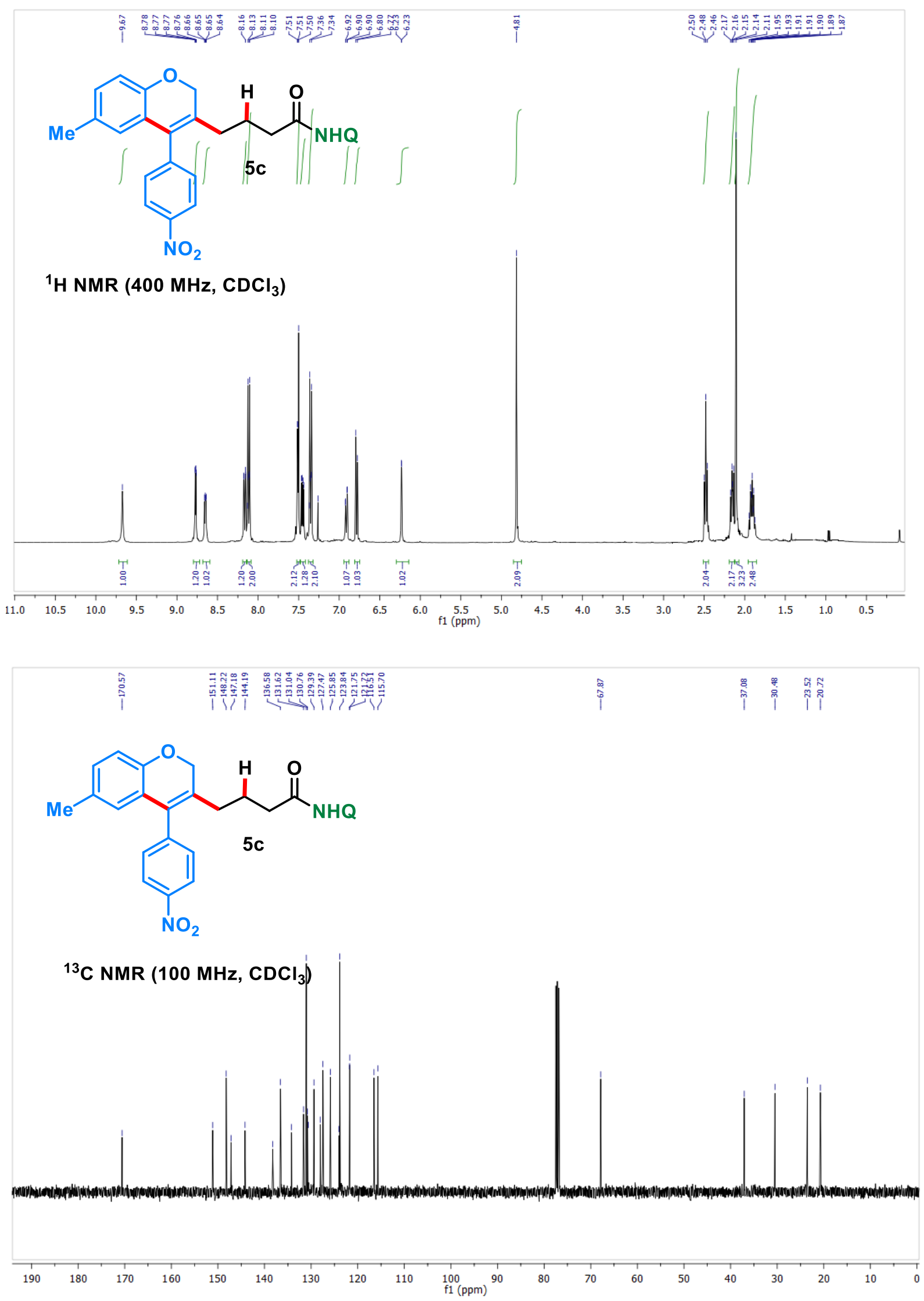


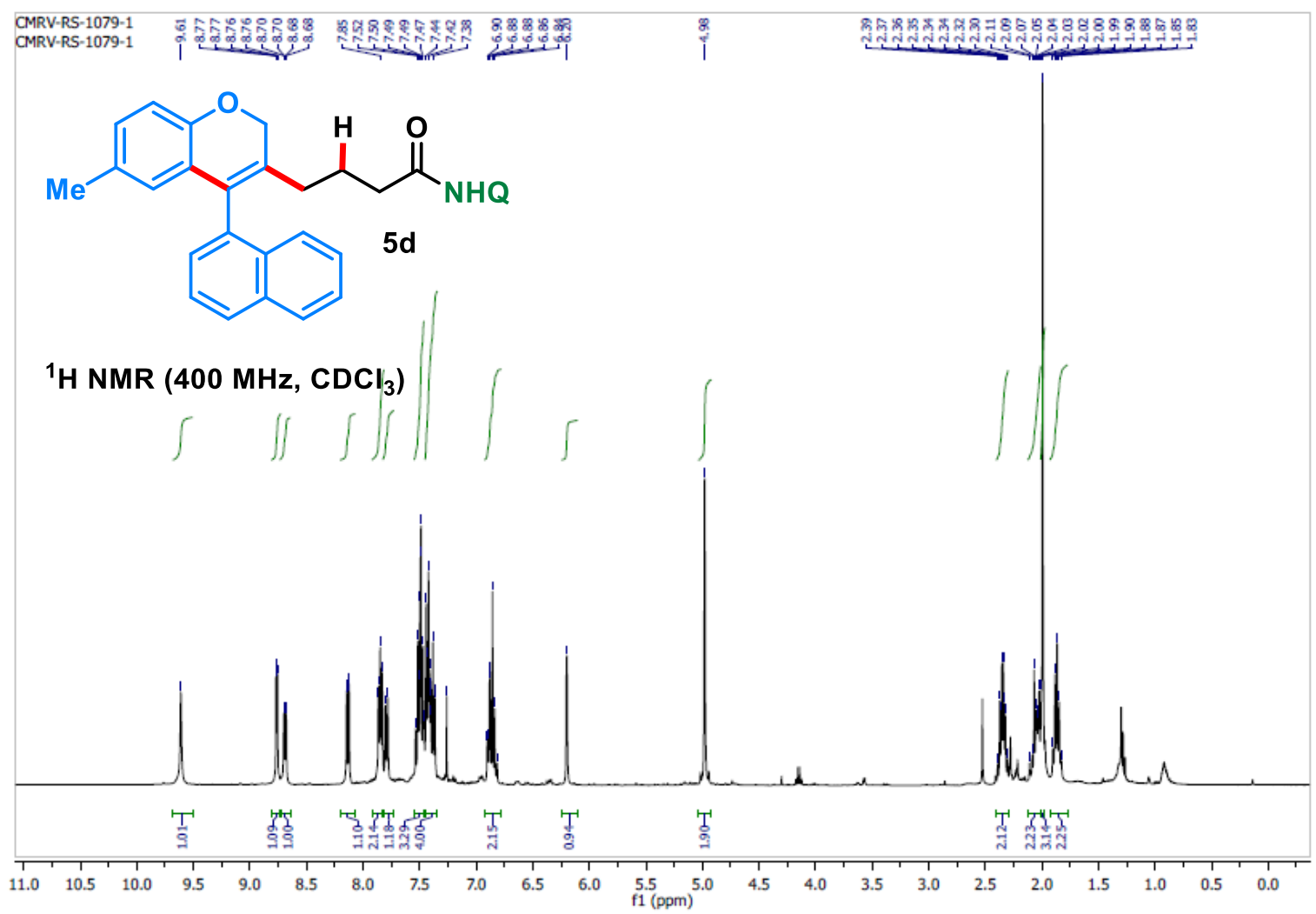

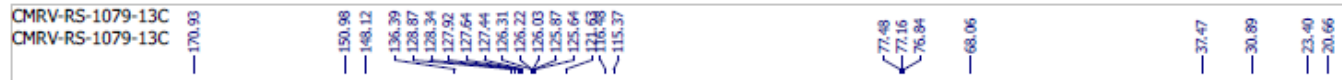<smiles>Cc1ccc2c(c1)C(c1cccc3ccccc13)=C(CCC(=O)NO)CO2</smiles>

${ }^{13} \mathrm{C}$ NMR $\left(100 \mathrm{MHz}, \mathrm{CDCl}_{3}\right)$
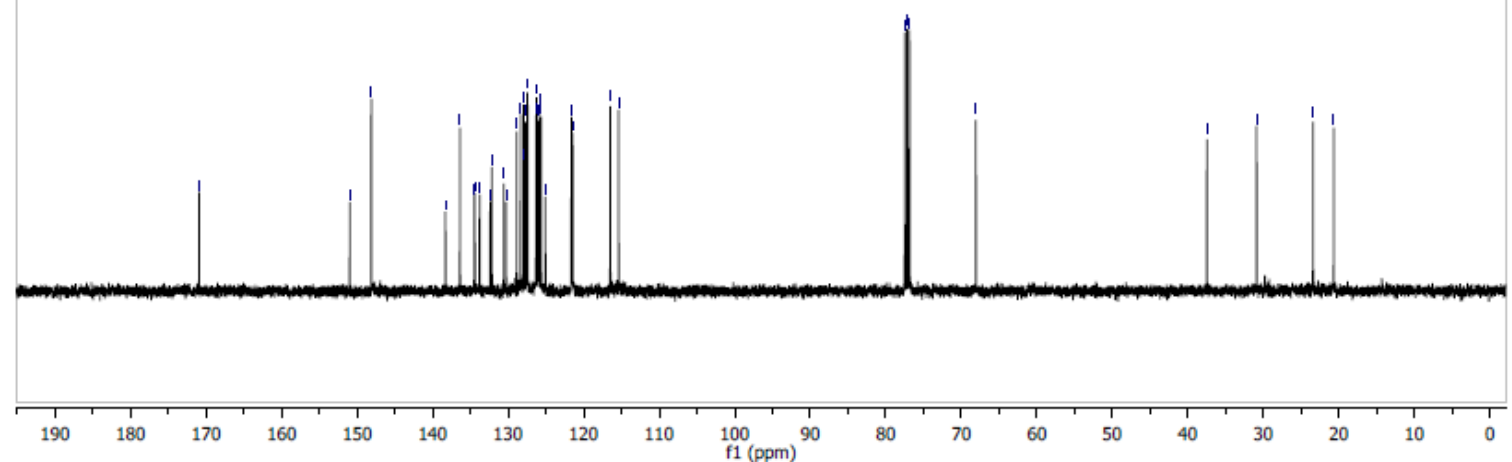

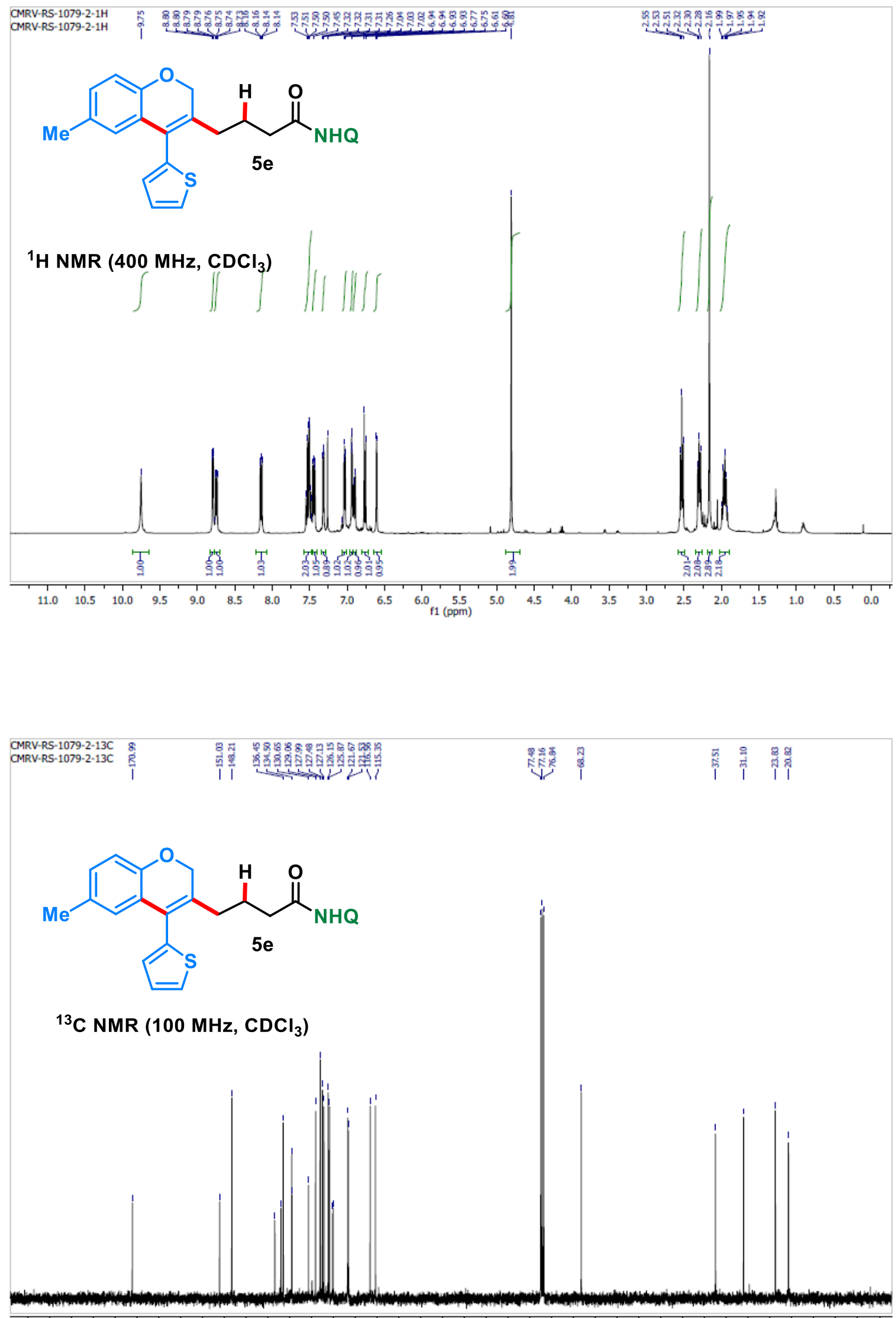

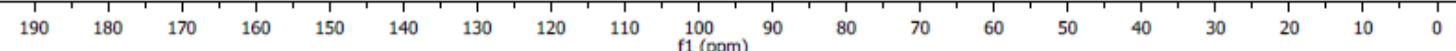



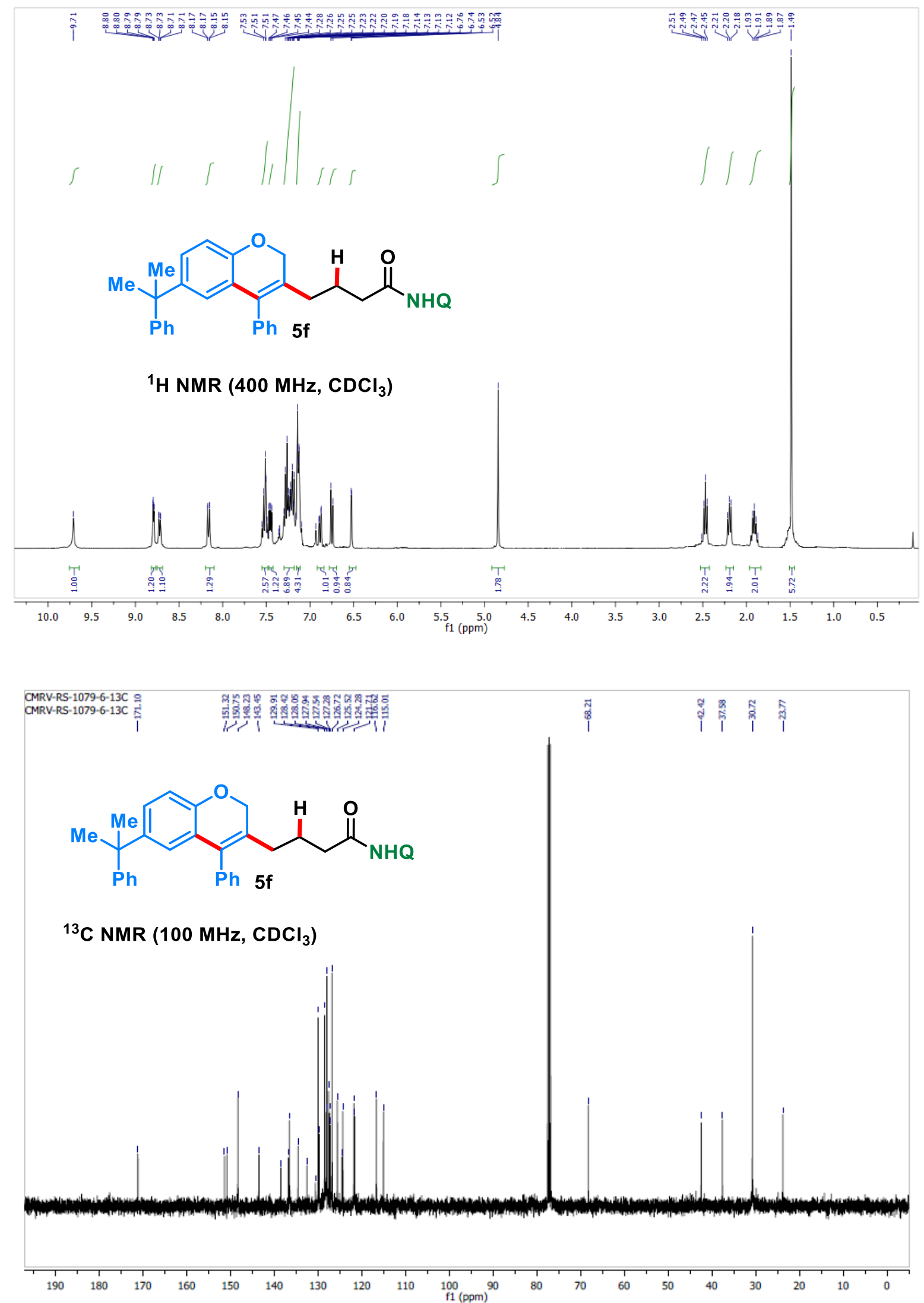

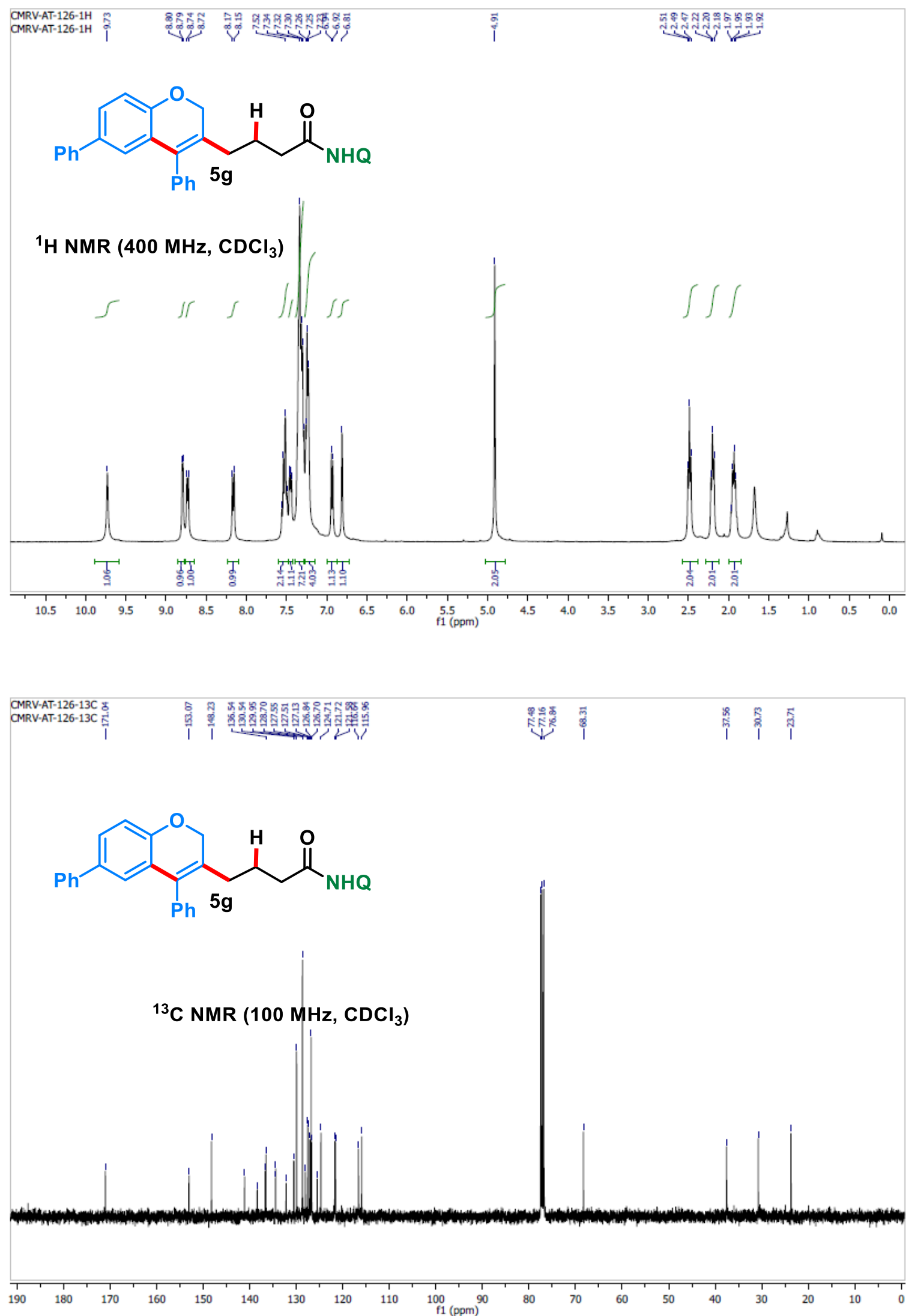

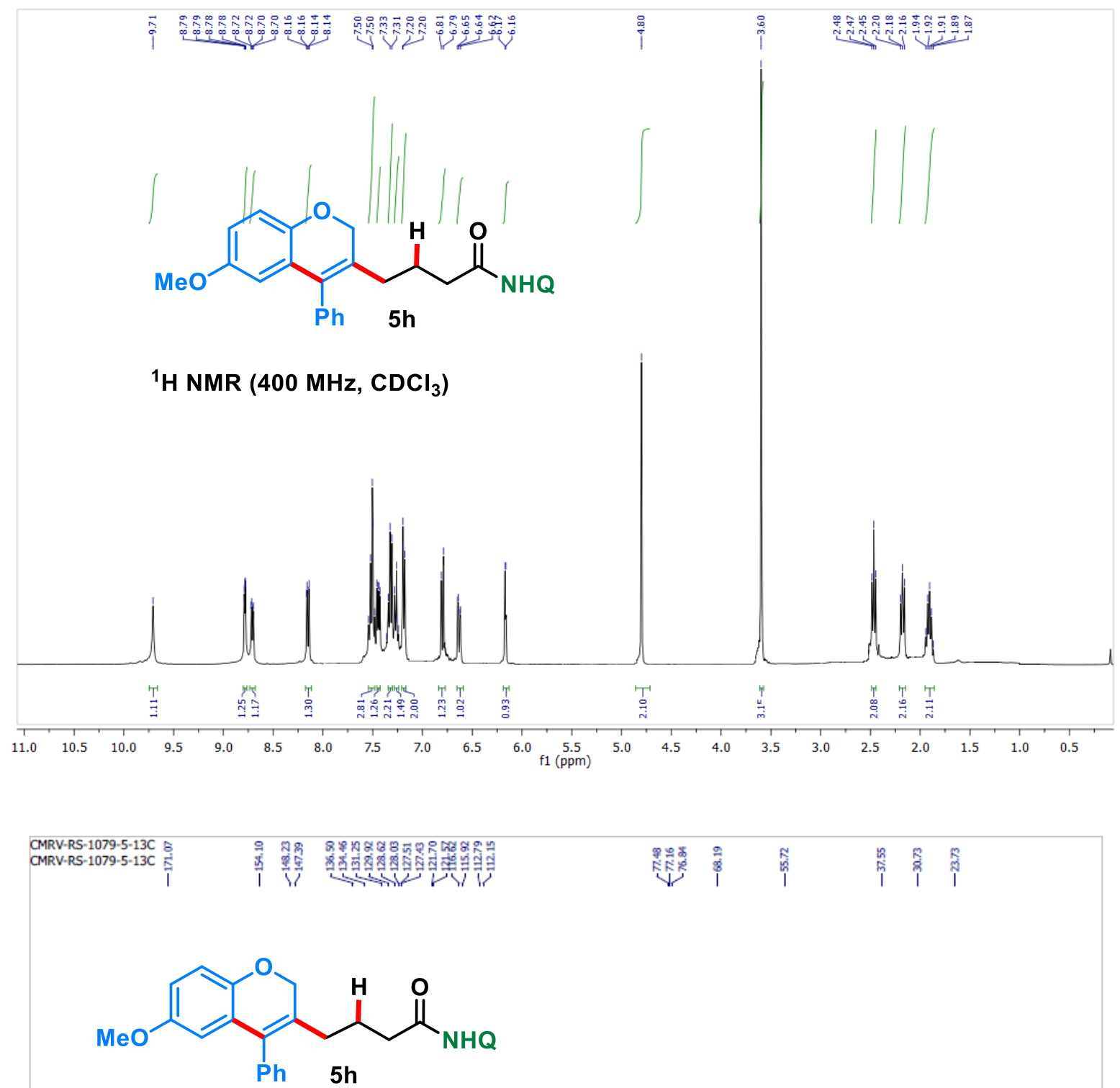

${ }^{13} \mathrm{C}$ NMR $\left(100 \mathrm{MHz}, \mathrm{CDCl}_{3}\right)$
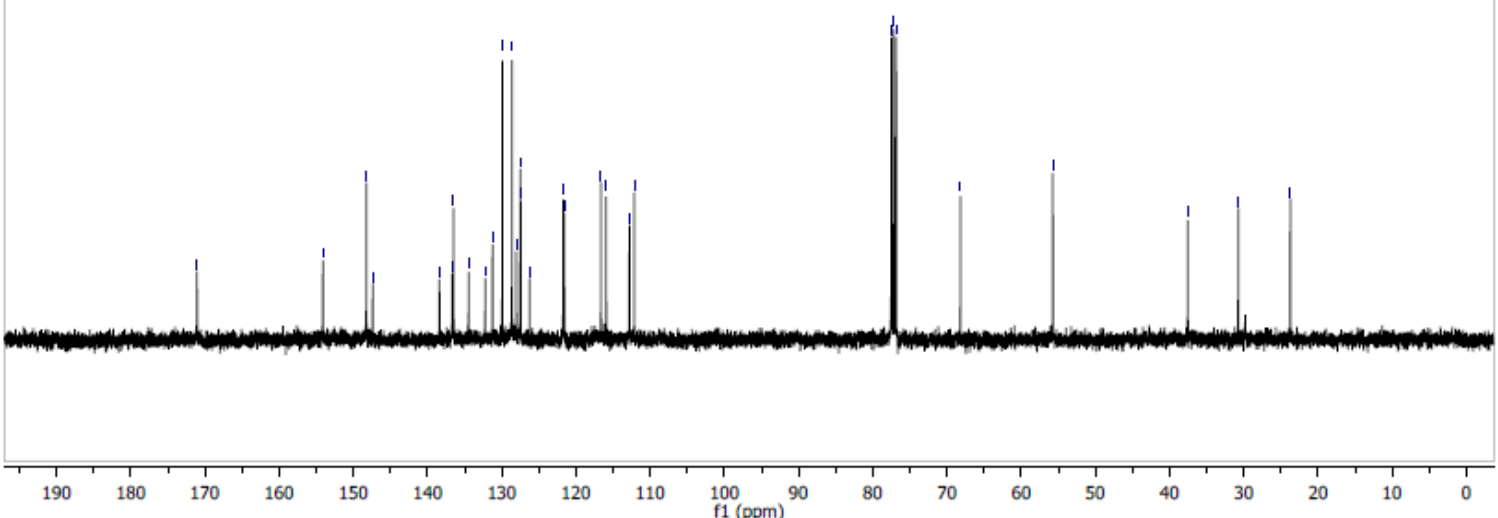

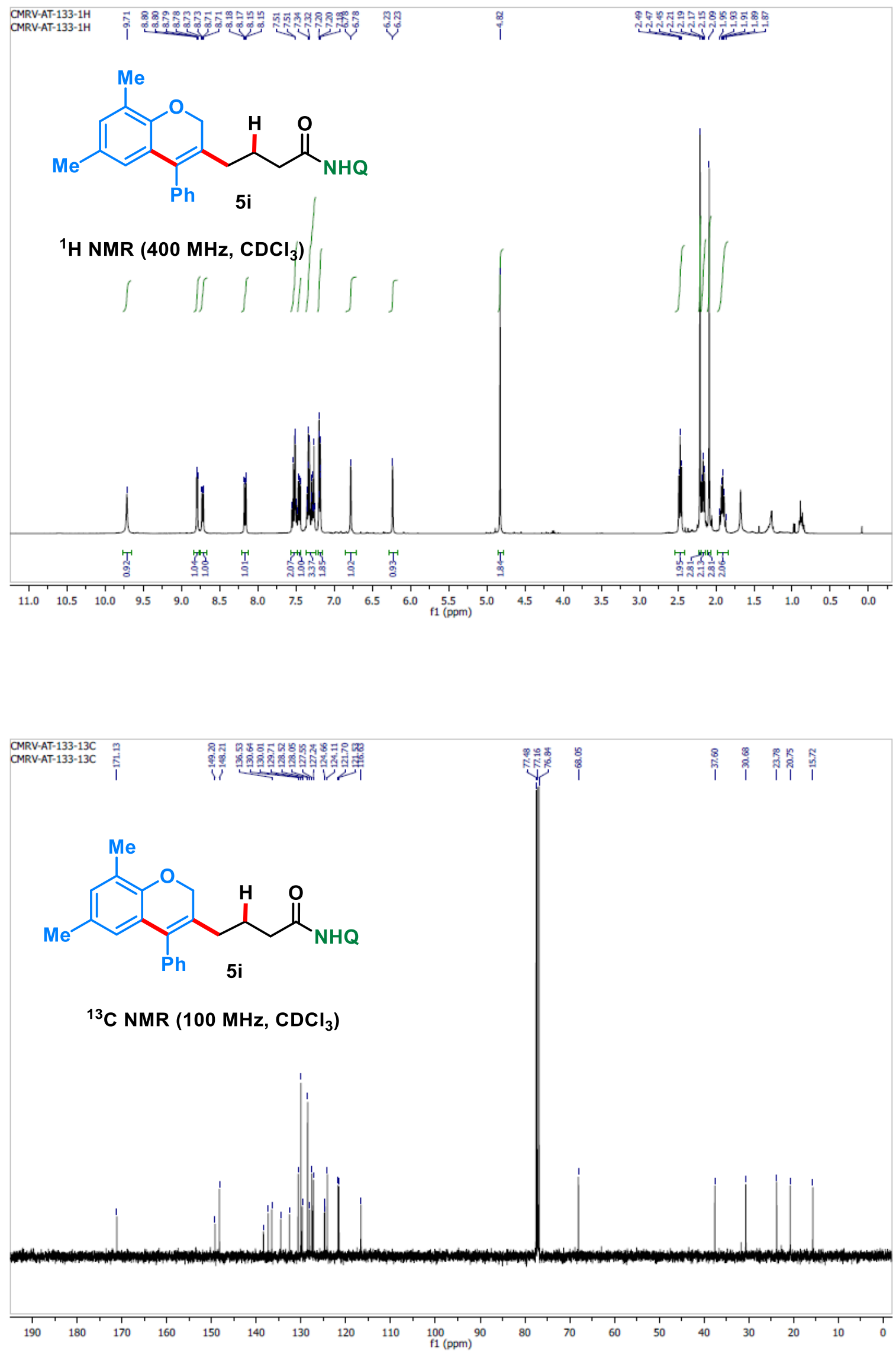
<smiles>O=C(CC1=C(c2ccccc2)c2c(ccc3ccccc23)OCC1CC(=O)NO)NO</smiles>

${ }^{1} \mathrm{H}$ NMR $\left(400 \mathrm{MHz}, \mathrm{CDCl}_{3}\right)$
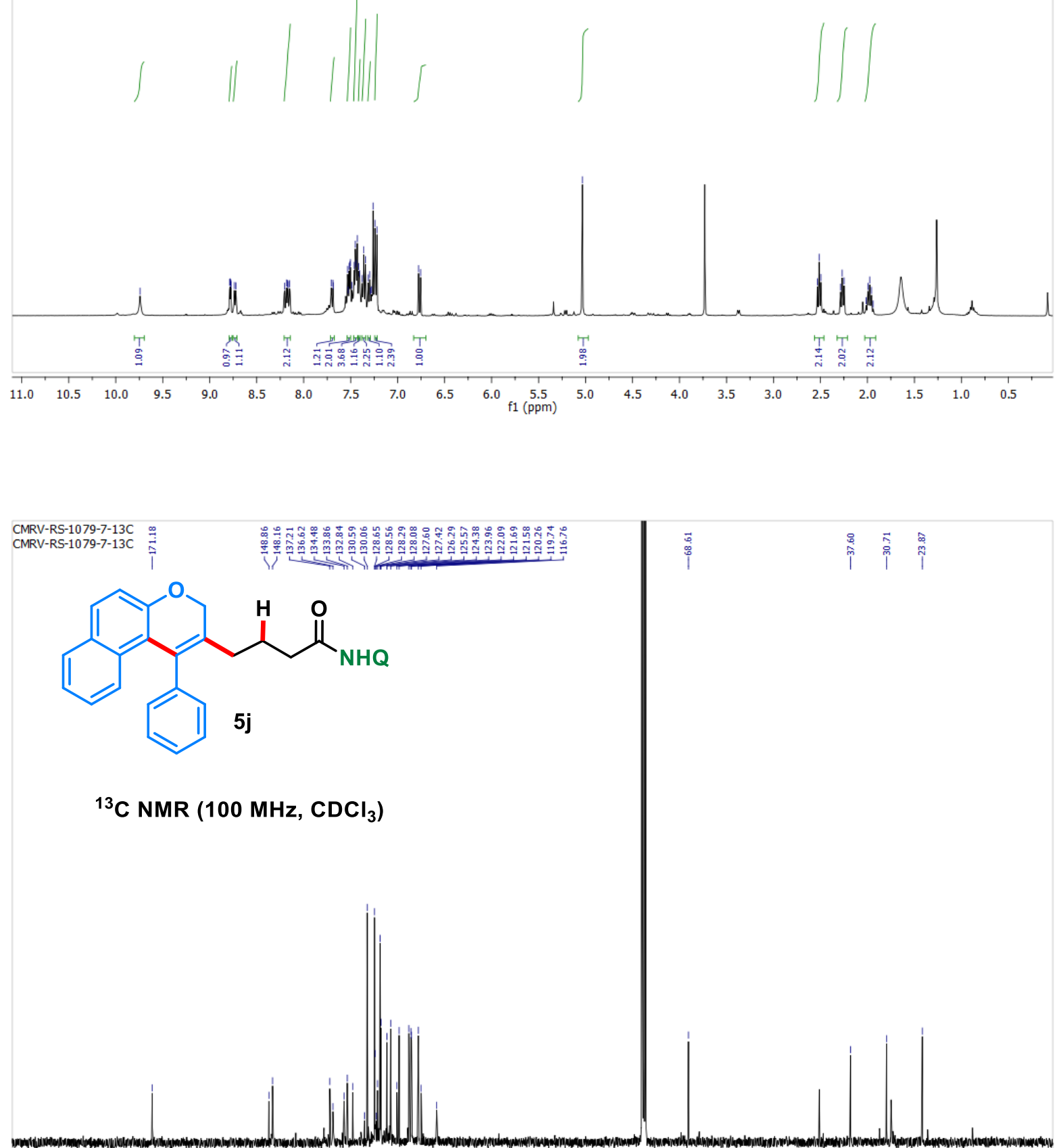

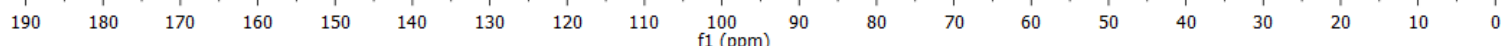



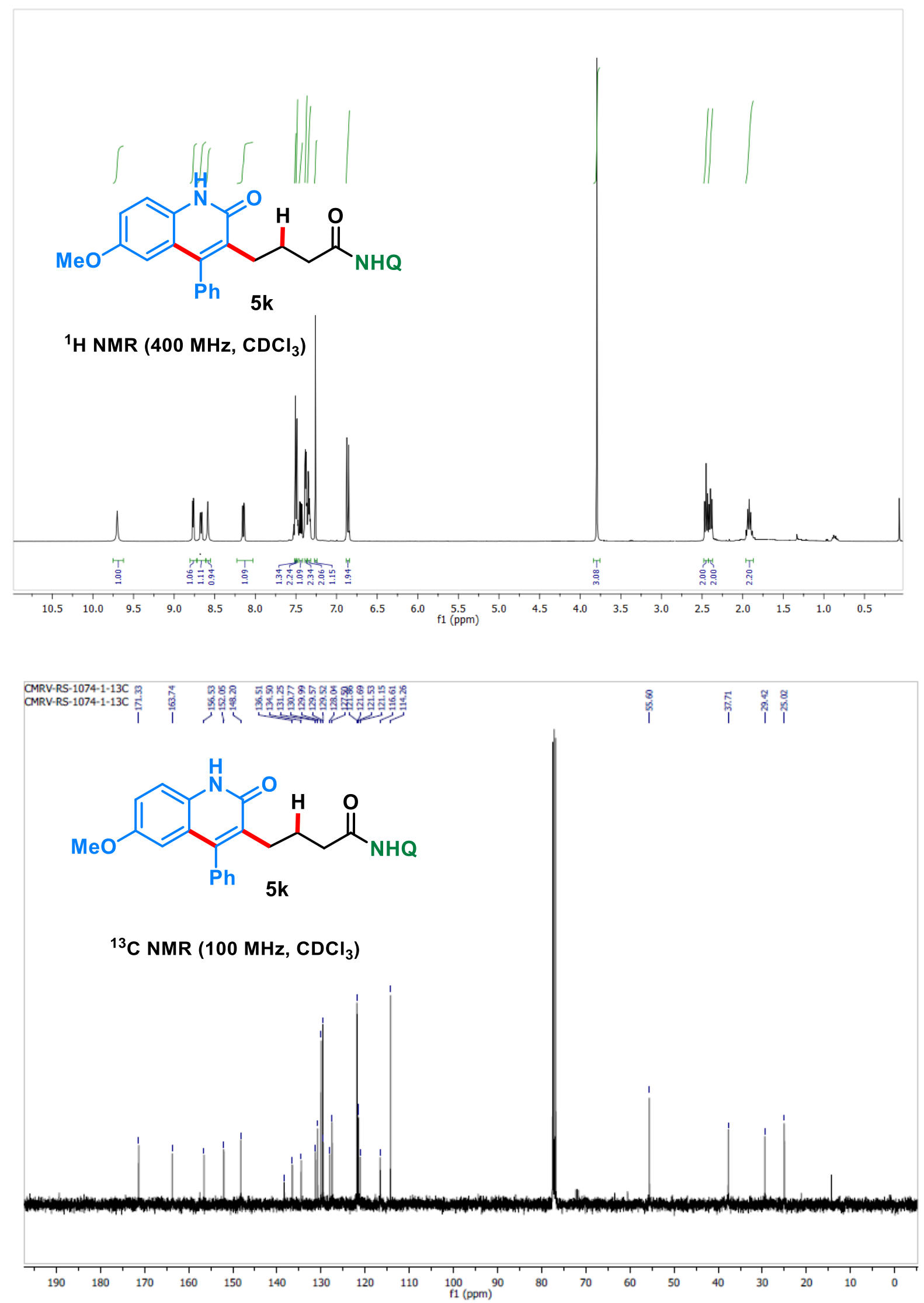


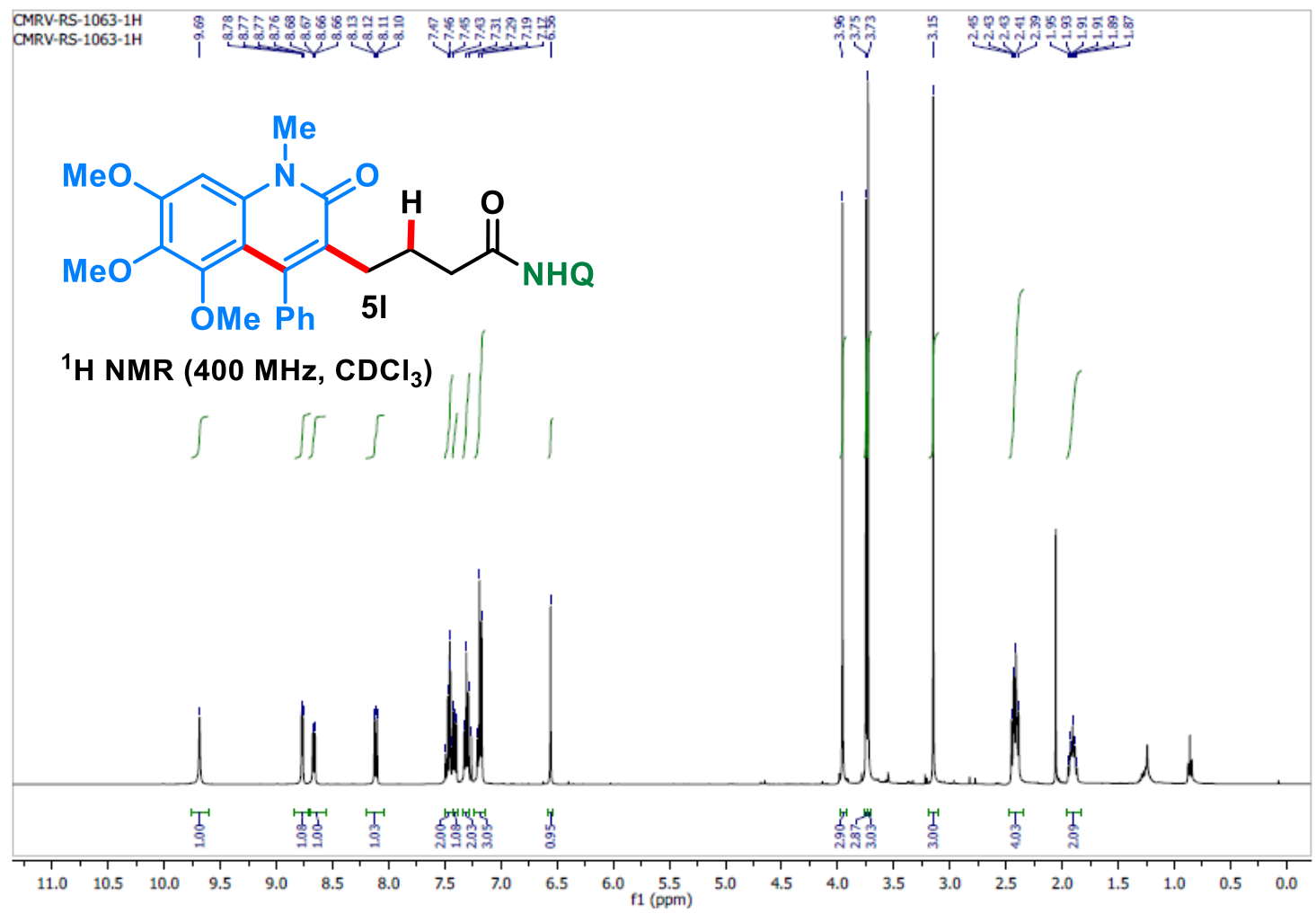

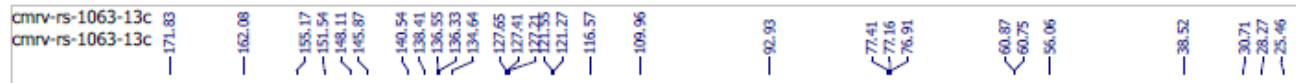<smiles>COc1cc2c(c(OC)c1OC)c(-c1ccccc1)c(CCC(=O)NO)c(=O)n2C</smiles>

${ }^{13} \mathrm{C}$ NMR (125 MHz, $\mathrm{CDCl}_{3}$ )
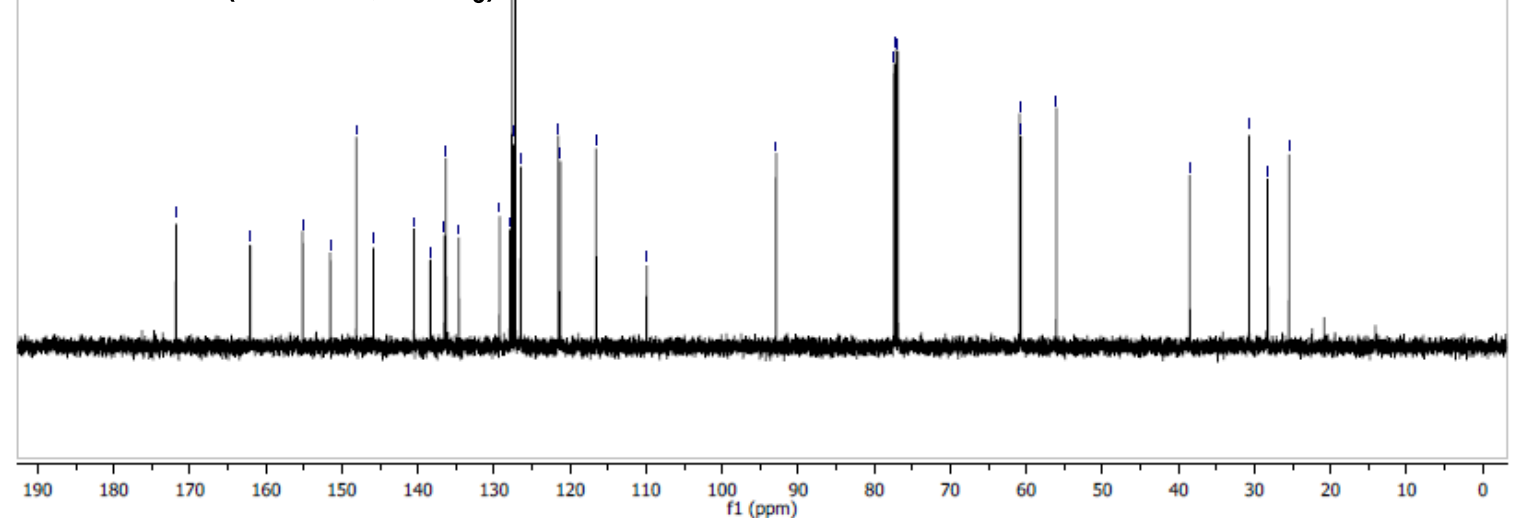


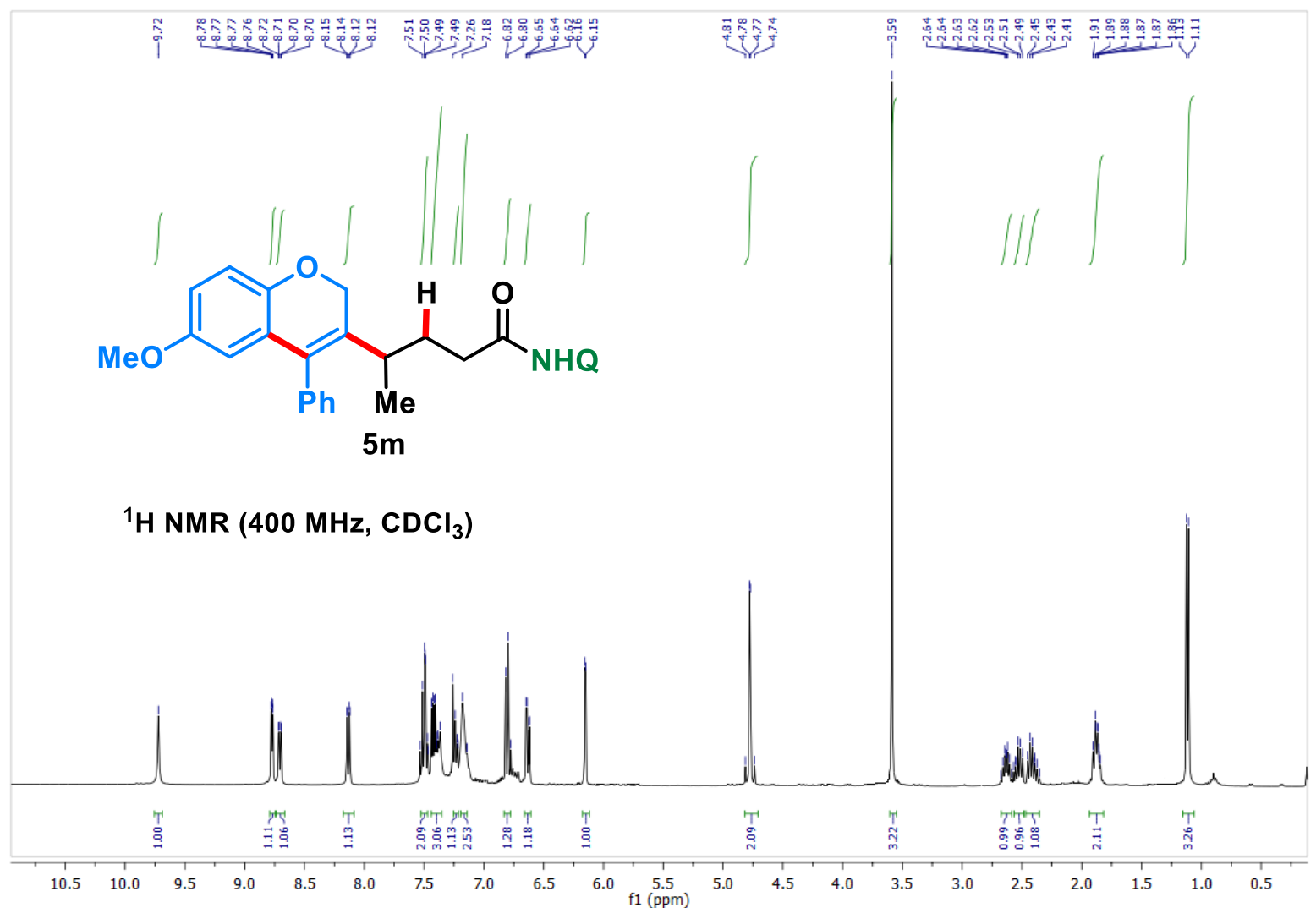

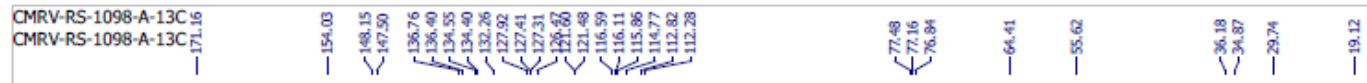<smiles>COc1ccc2c(c1)C(c1ccccc1)=C(C(C)CCC(=O)NO)CO2</smiles>

$5 \mathrm{~m}$

${ }^{13} \mathrm{C}$ NMR $\left(100 \mathrm{MHz}, \mathrm{CDCl}_{3}\right)$

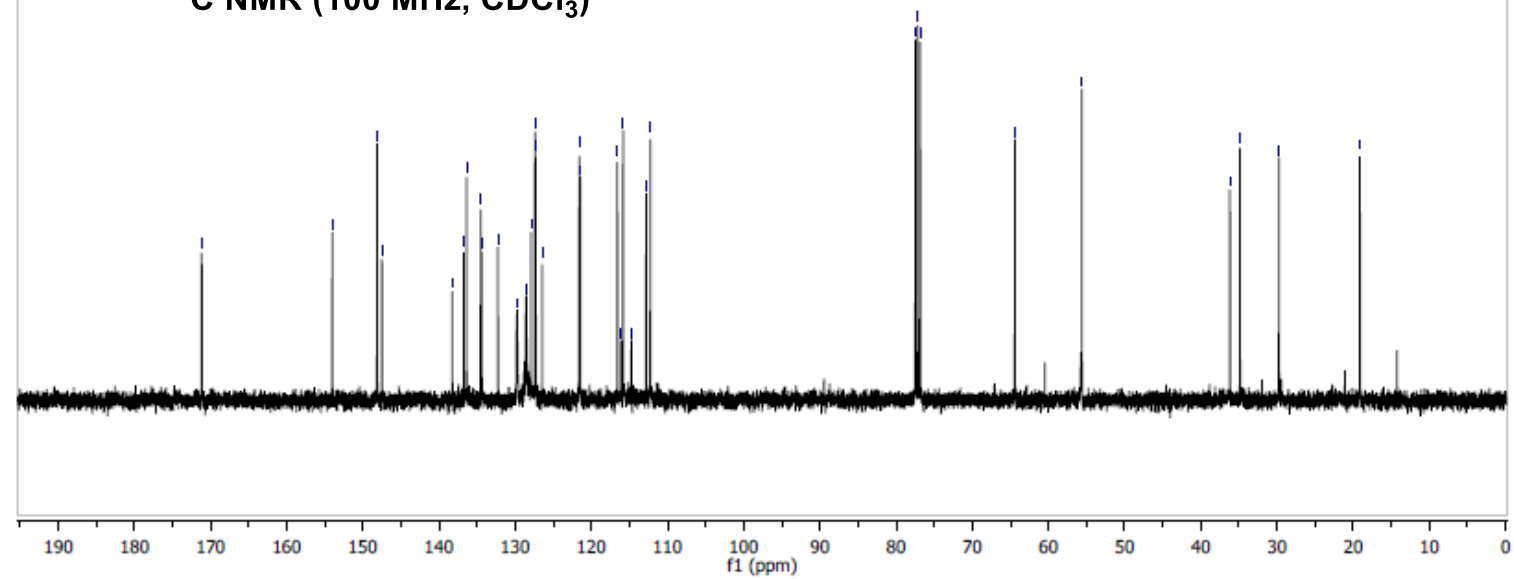



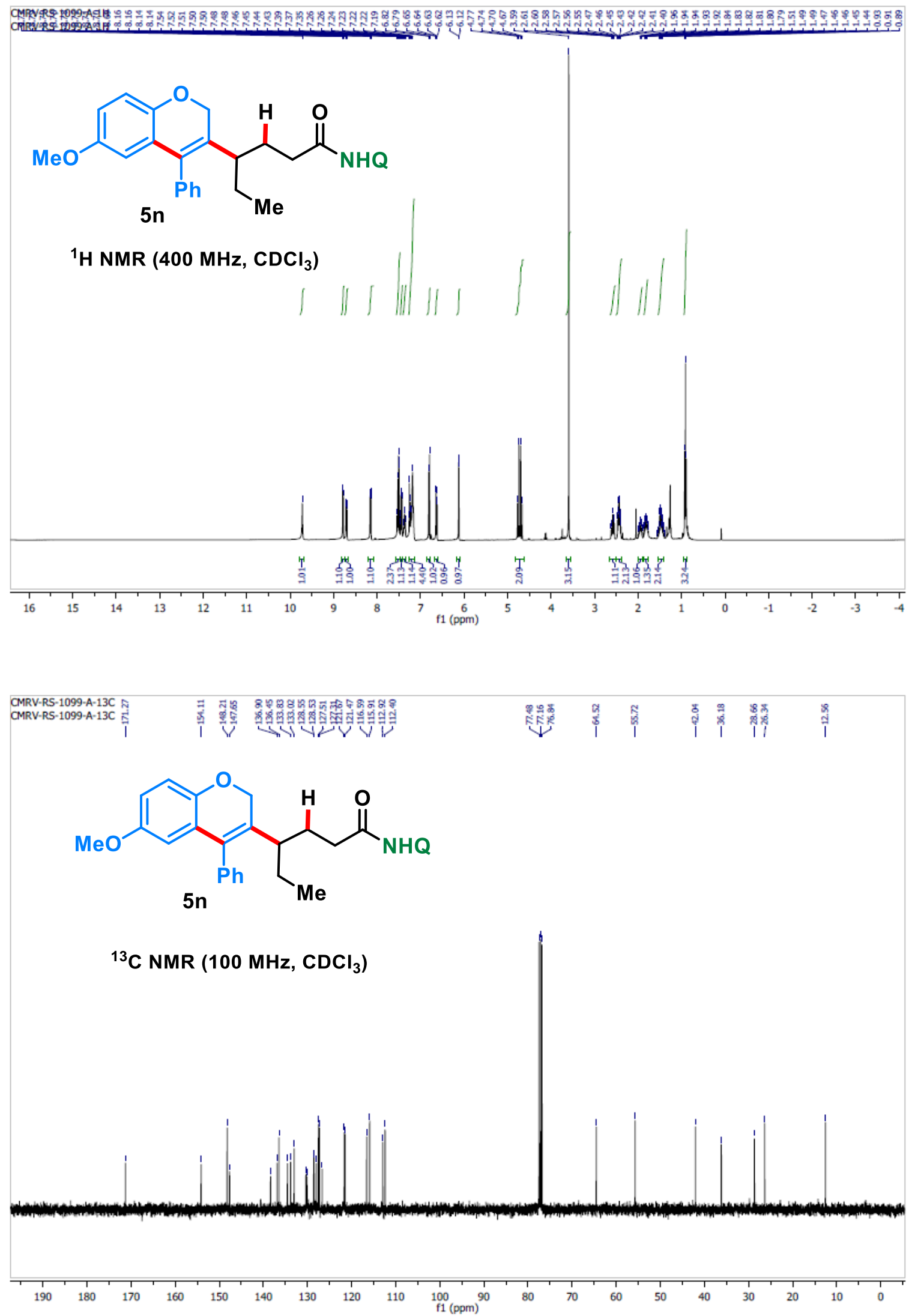

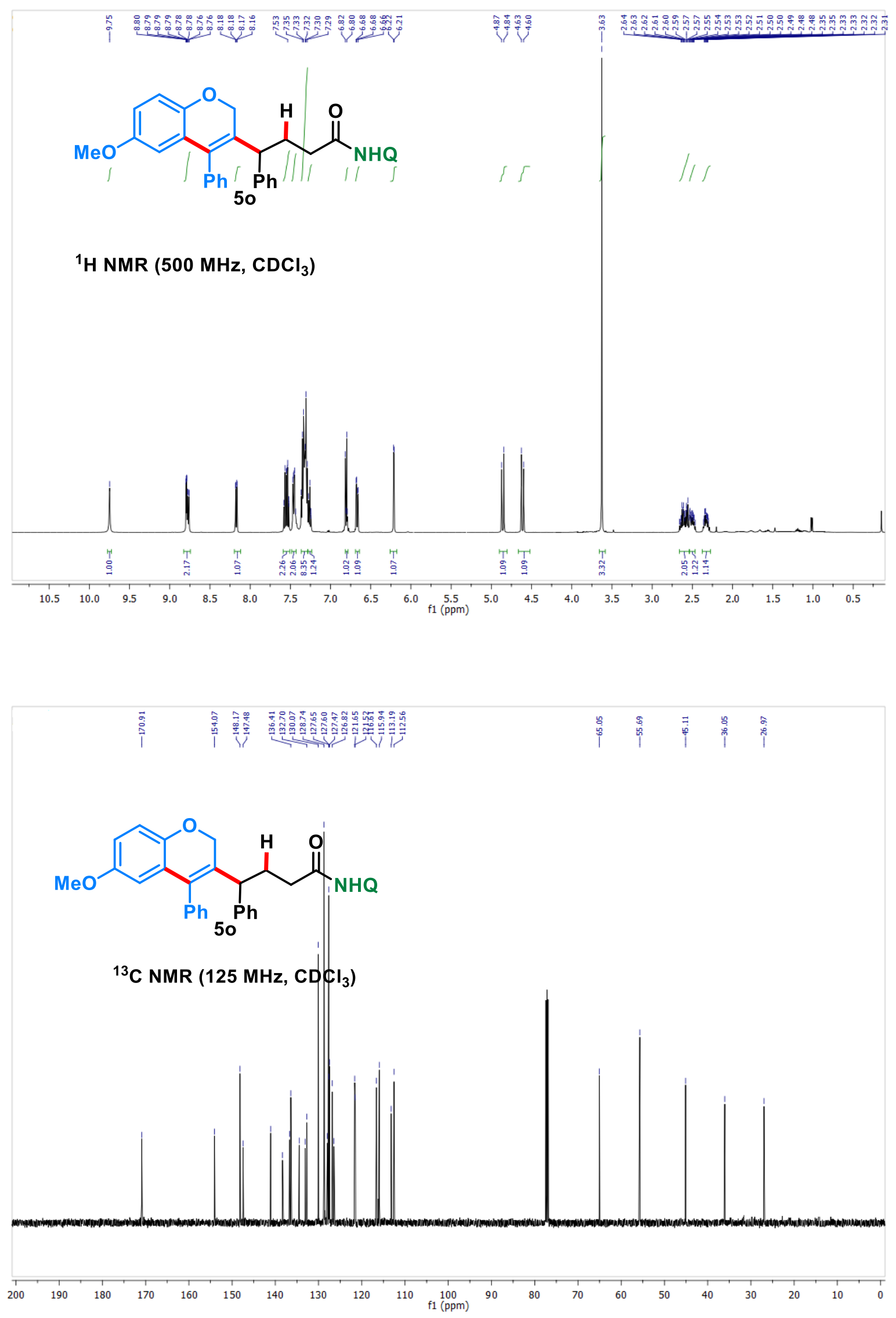

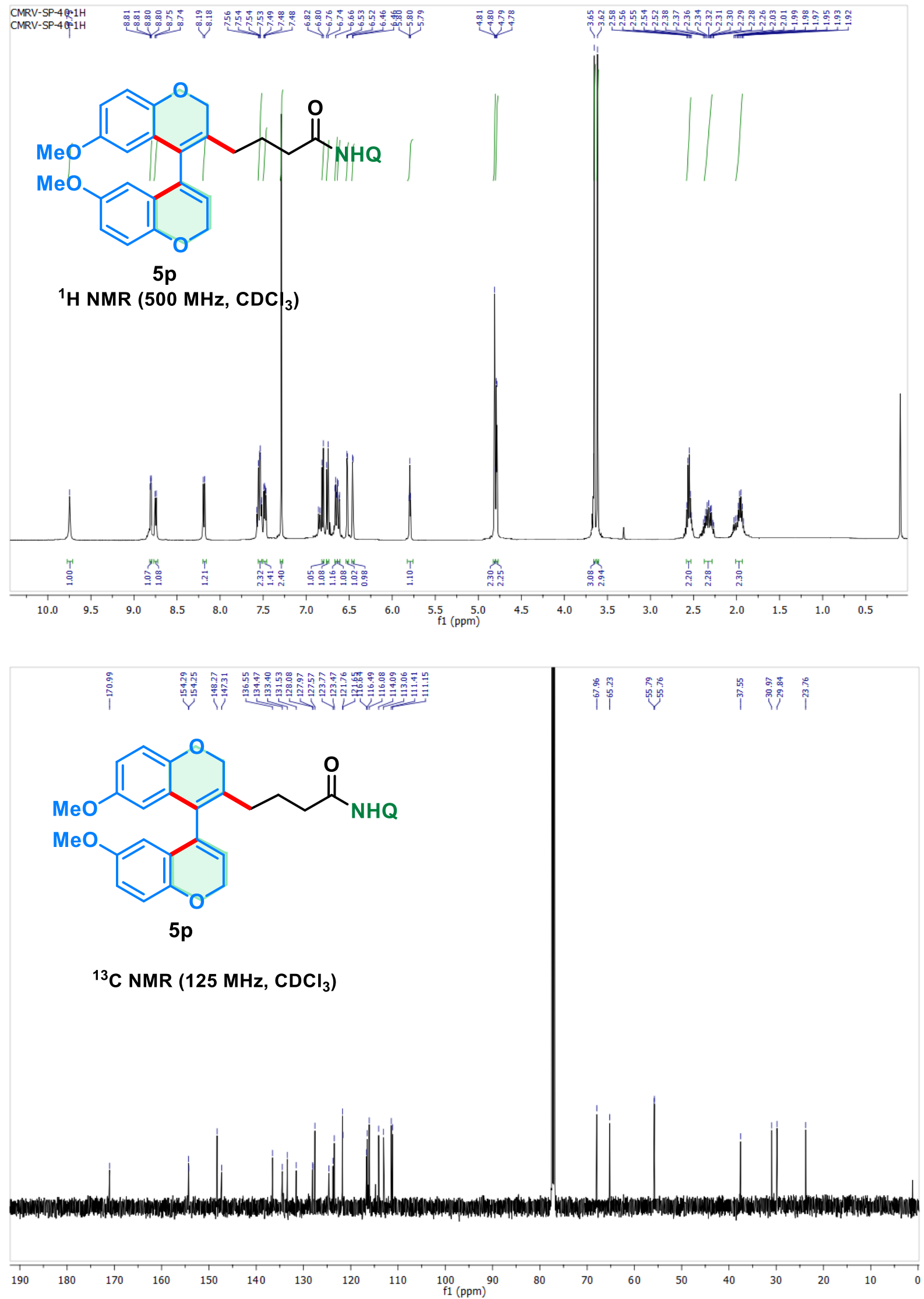

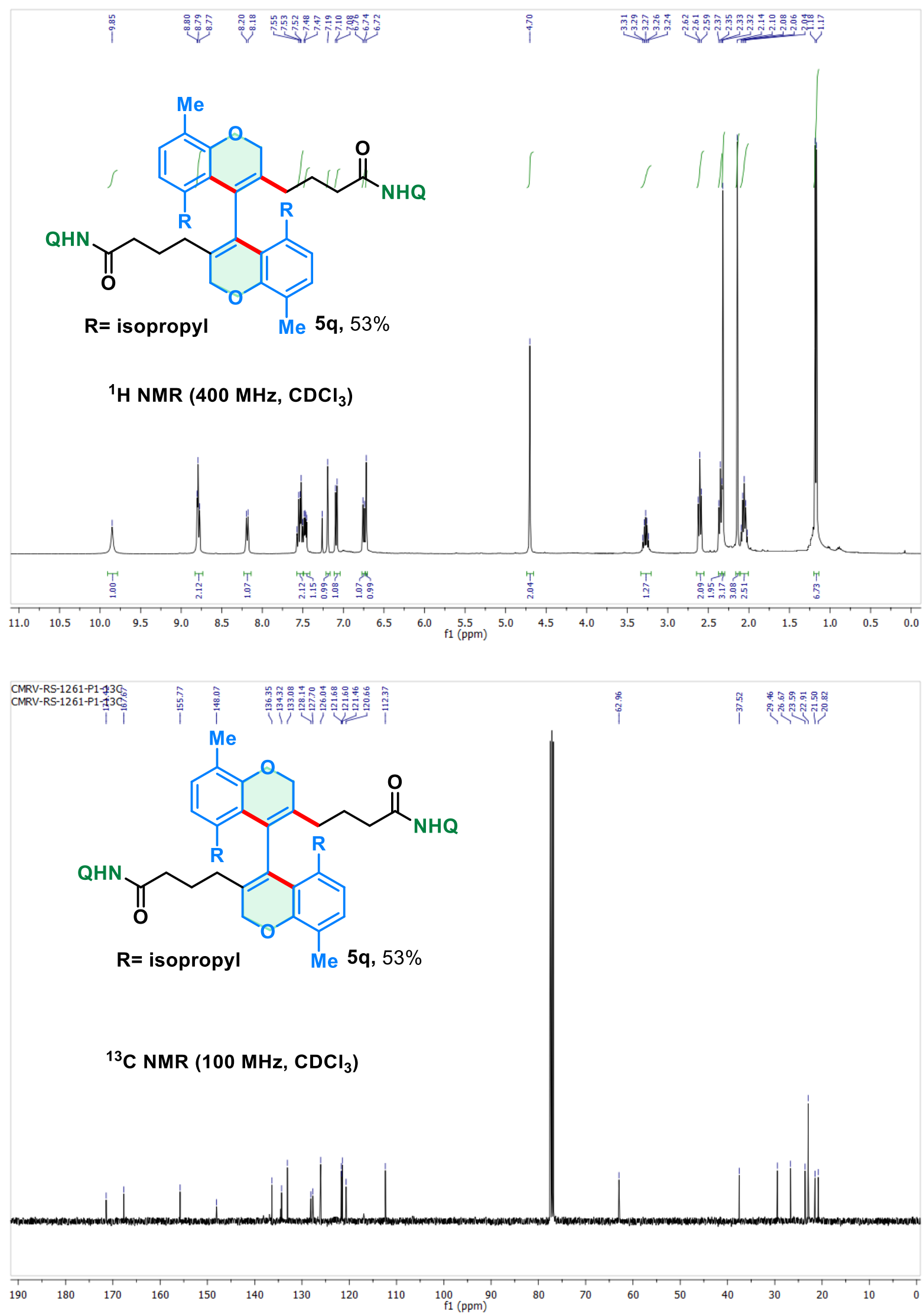

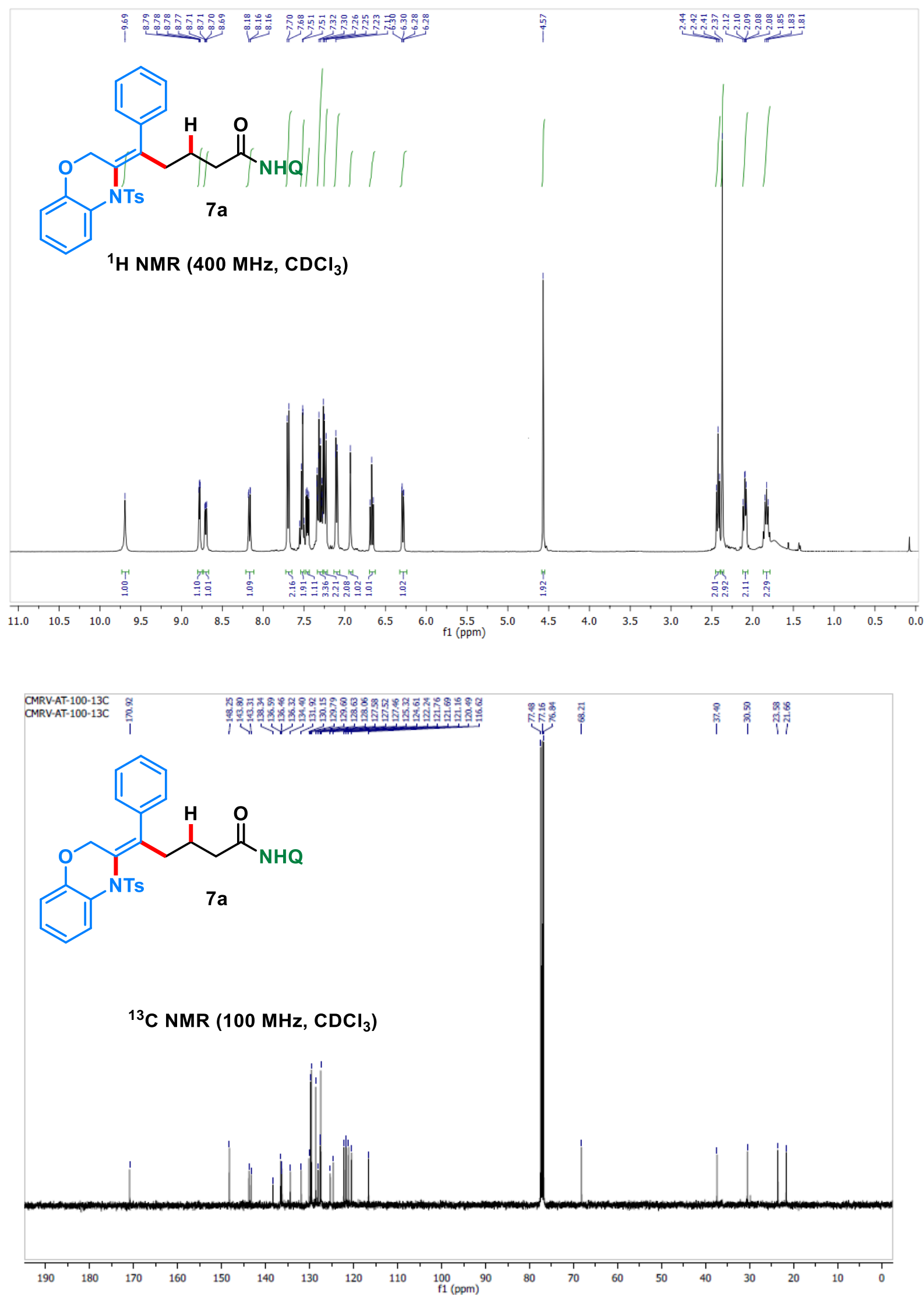


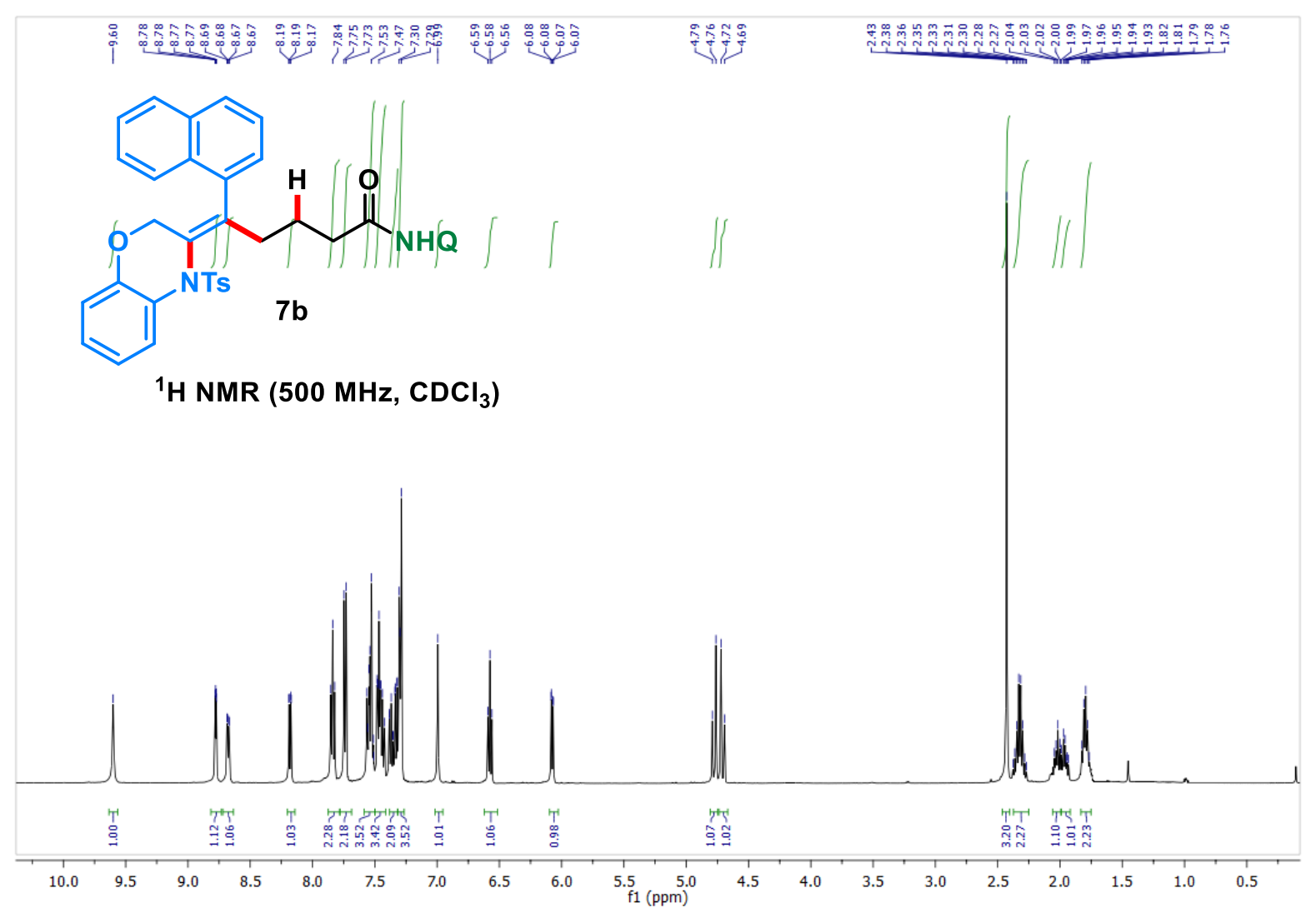

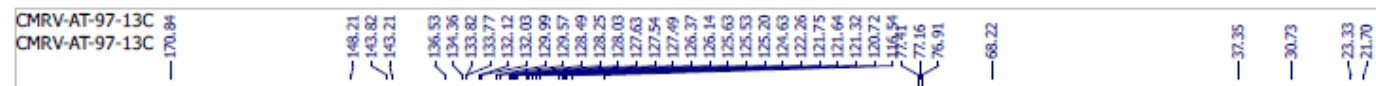<smiles>CC(C)(C)[Mg]</smiles>
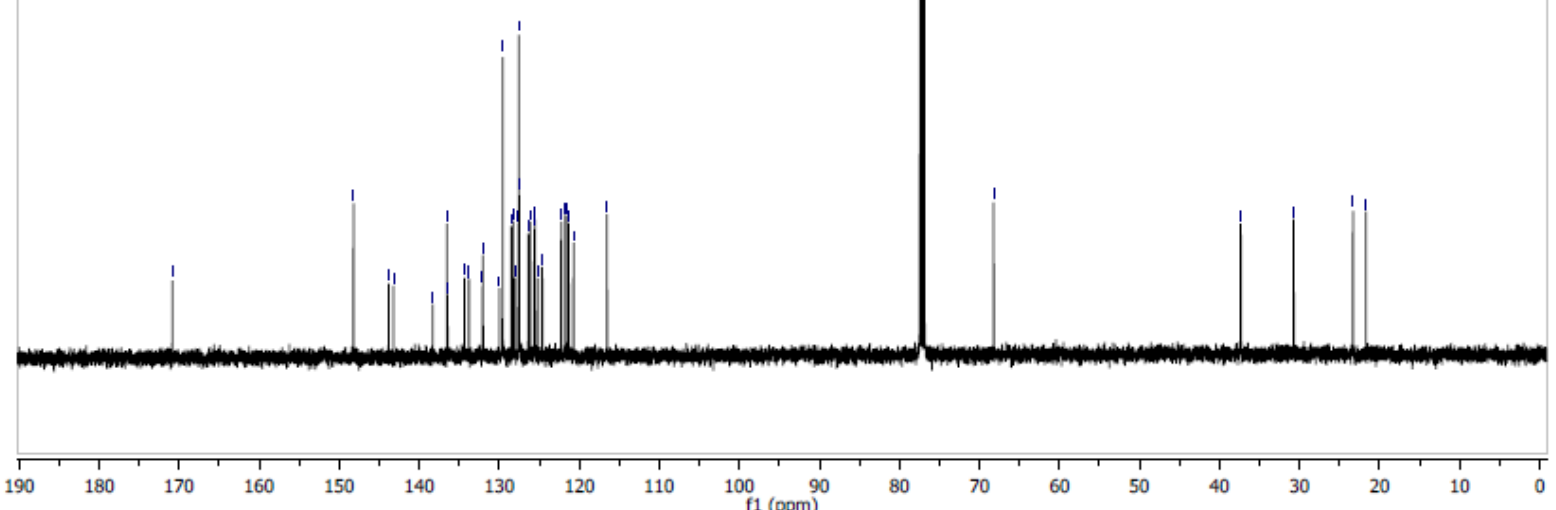


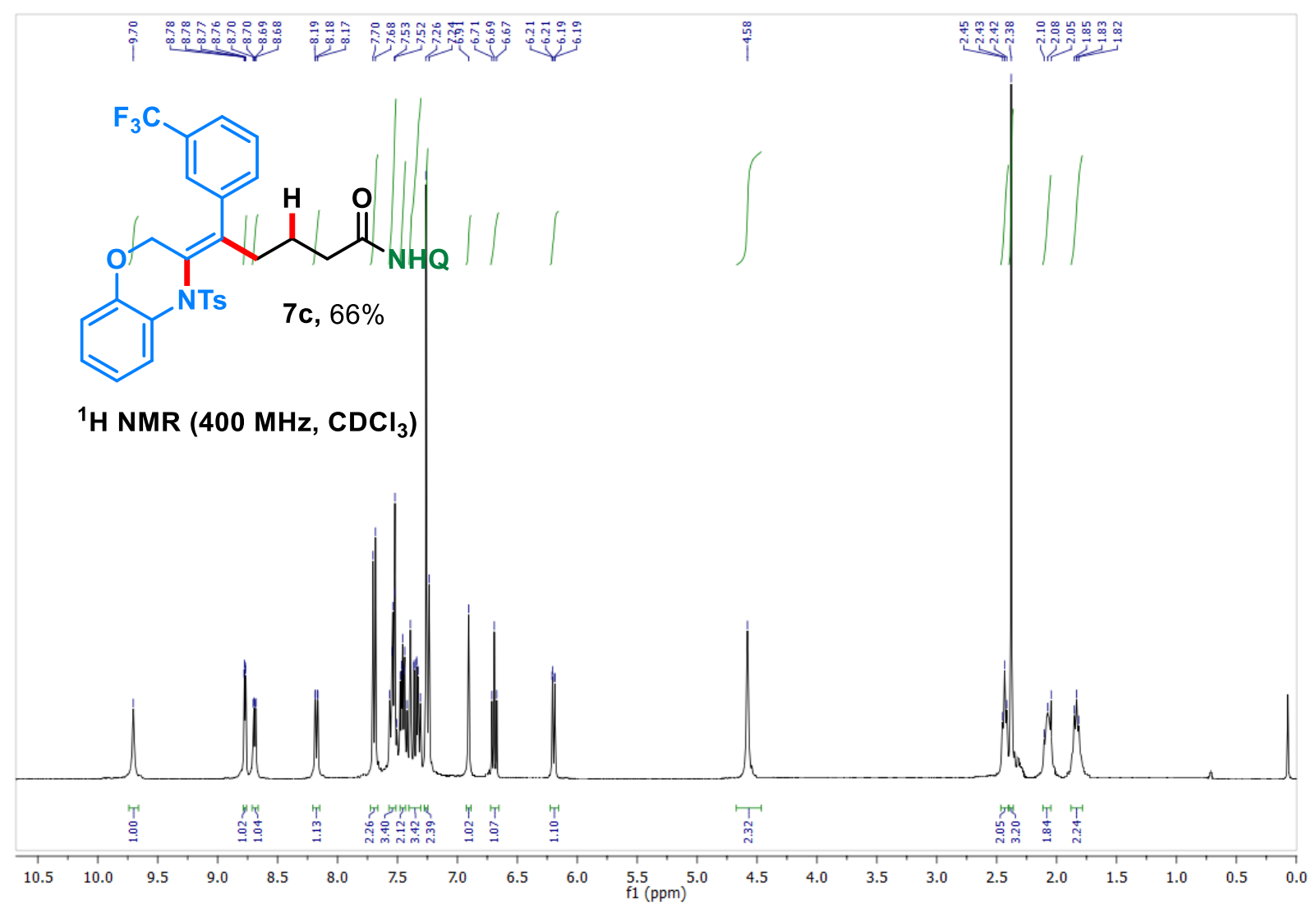

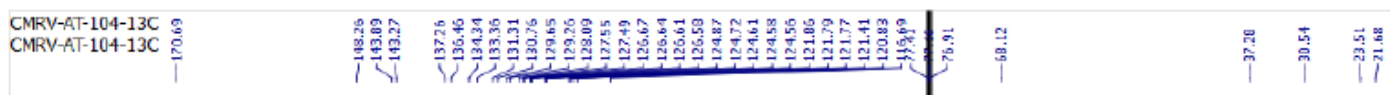

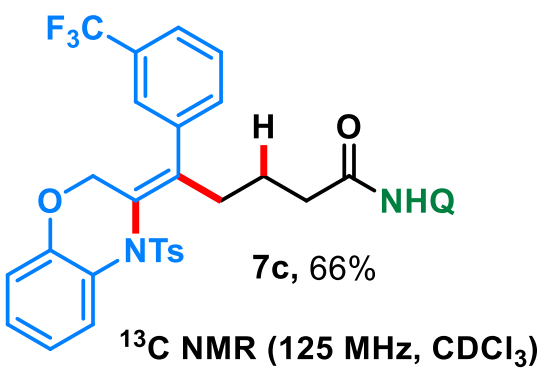

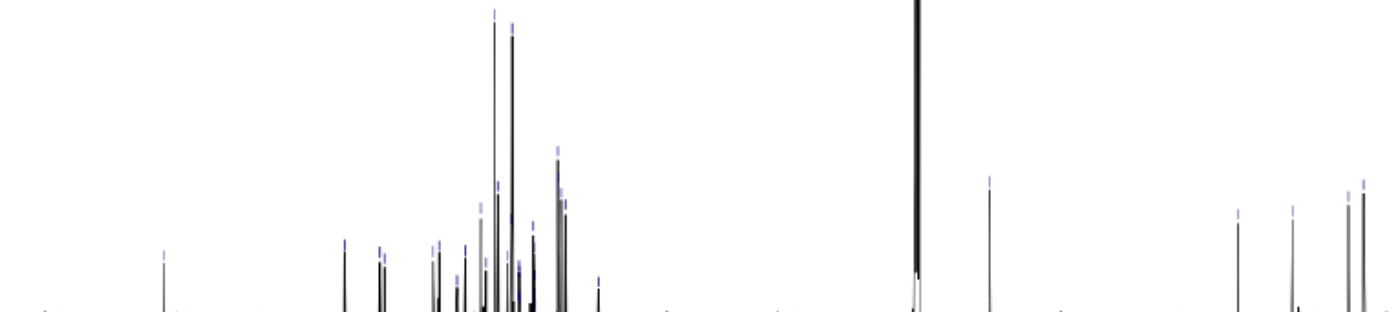

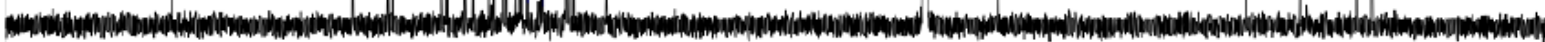

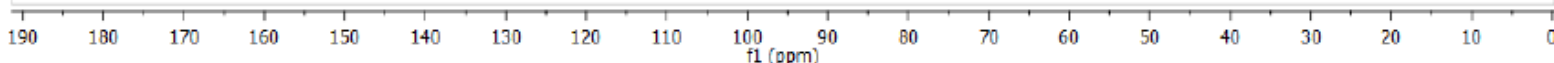




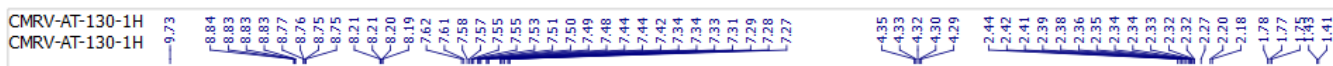<smiles>[3H]N1C(c2ccccc2)=C(CCC(N)=O)CC1C</smiles>

${ }^{1} \mathrm{H}$ NMR $\left(500 \mathrm{MHz}, \mathrm{CDCl}_{3}\right)$
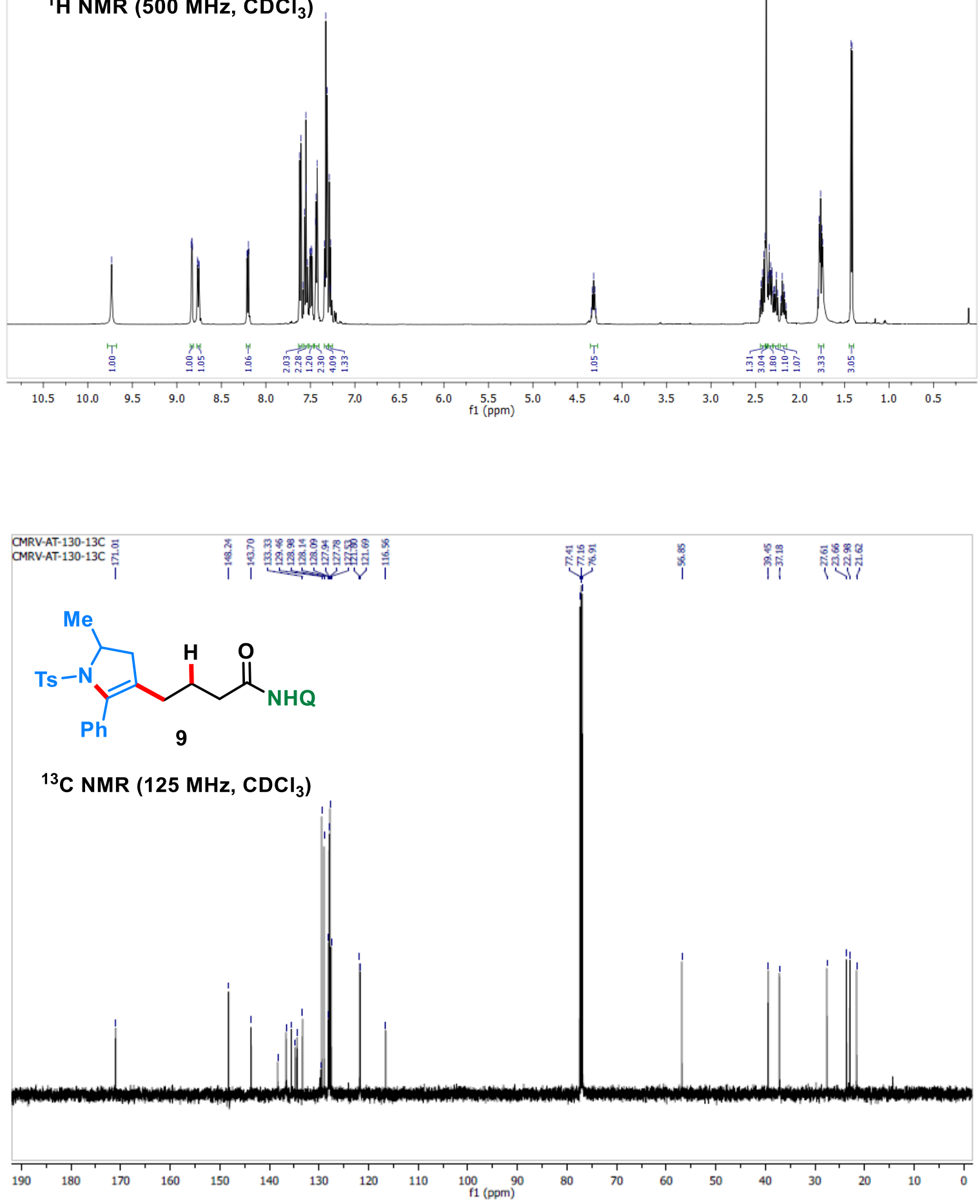

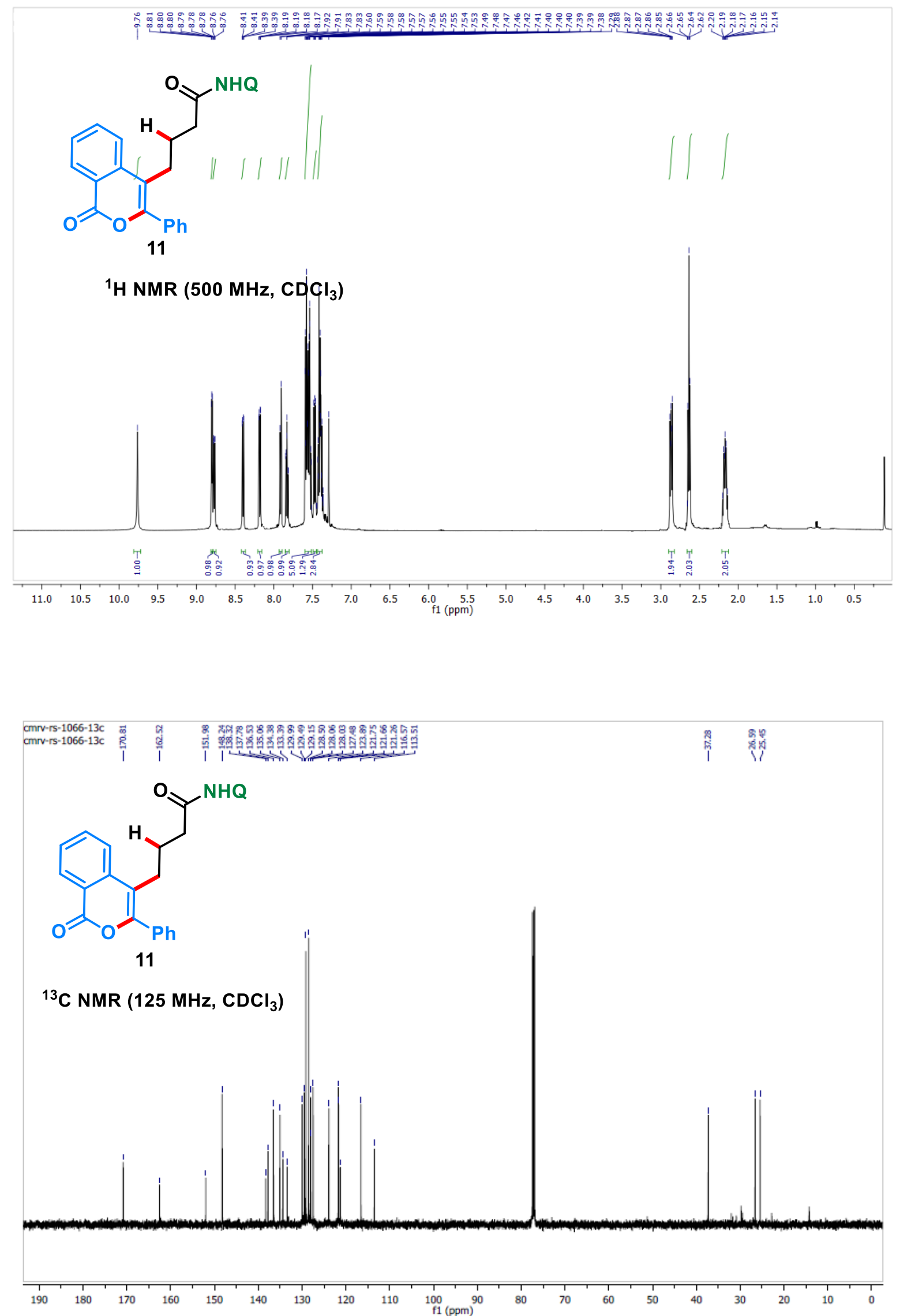

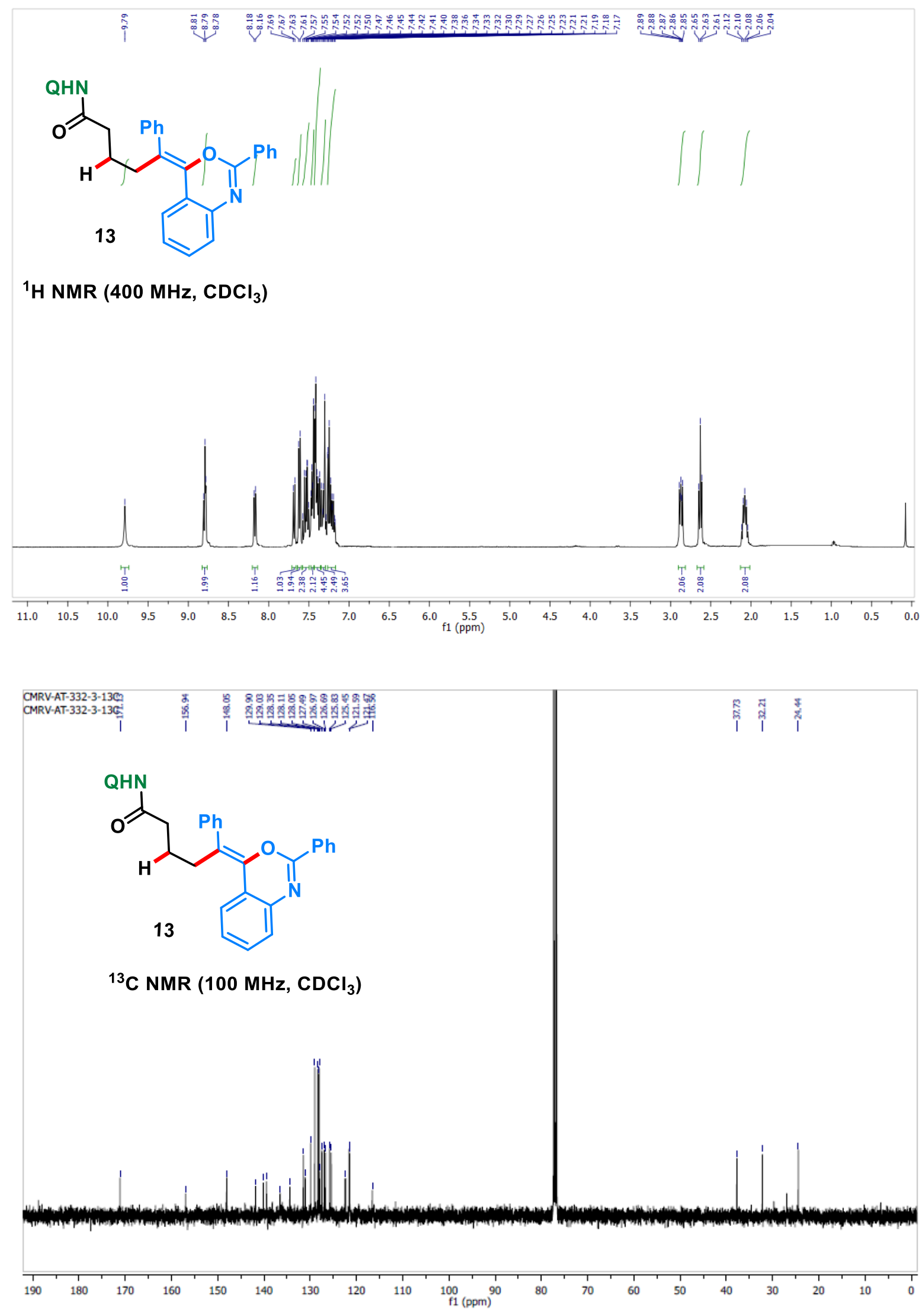

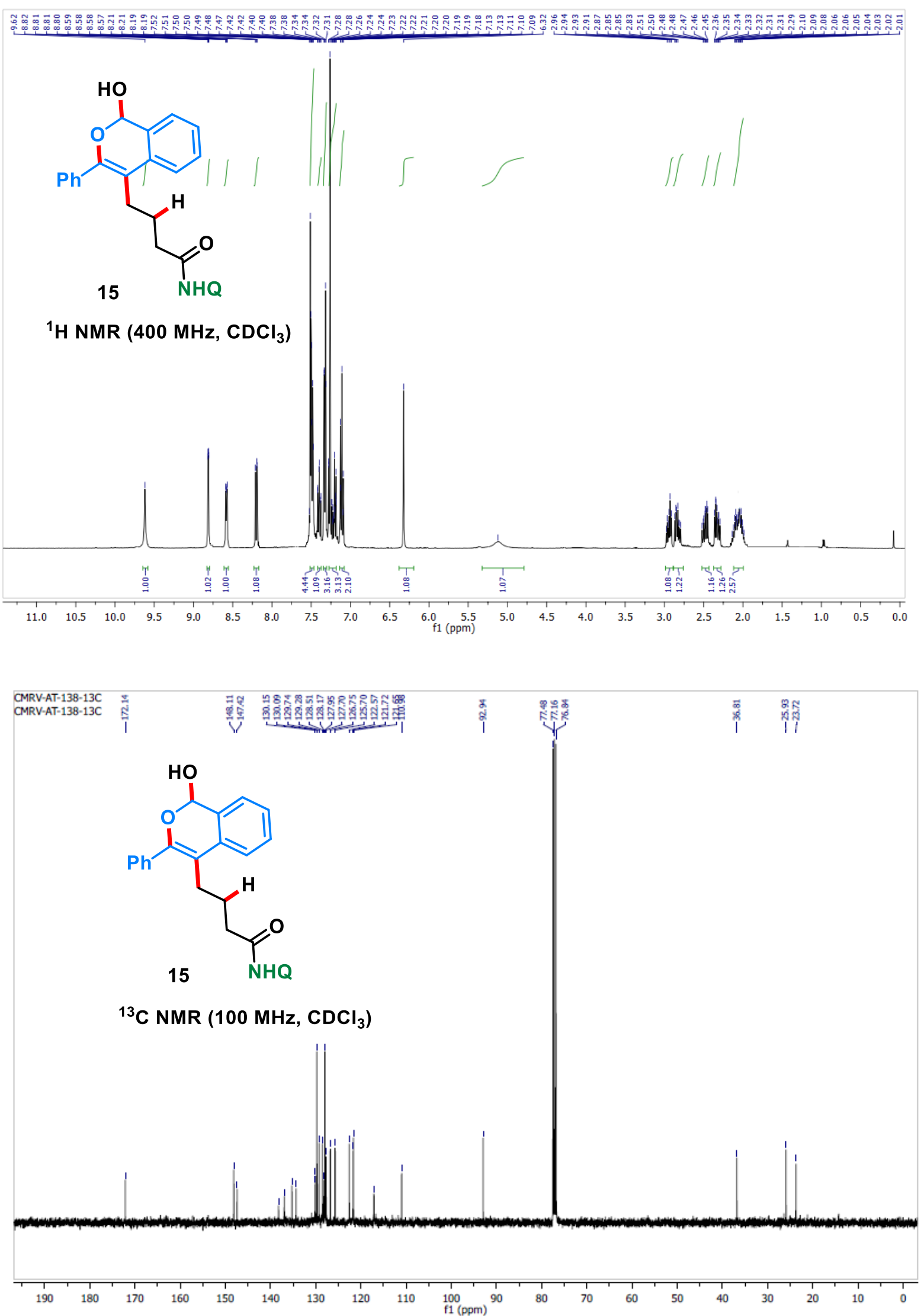
CMRV-AT-153-1H
CMRV-AT-153-1H

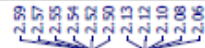<smiles>O=C1C=CC2(C=C1)OC(=O)C(CCC(=O)NO)=C2c1ccccc1</smiles>

${ }^{1} \mathrm{H}$ NMR $\left(400 \mathrm{MHz}, \mathrm{CDCl}_{3}\right)$
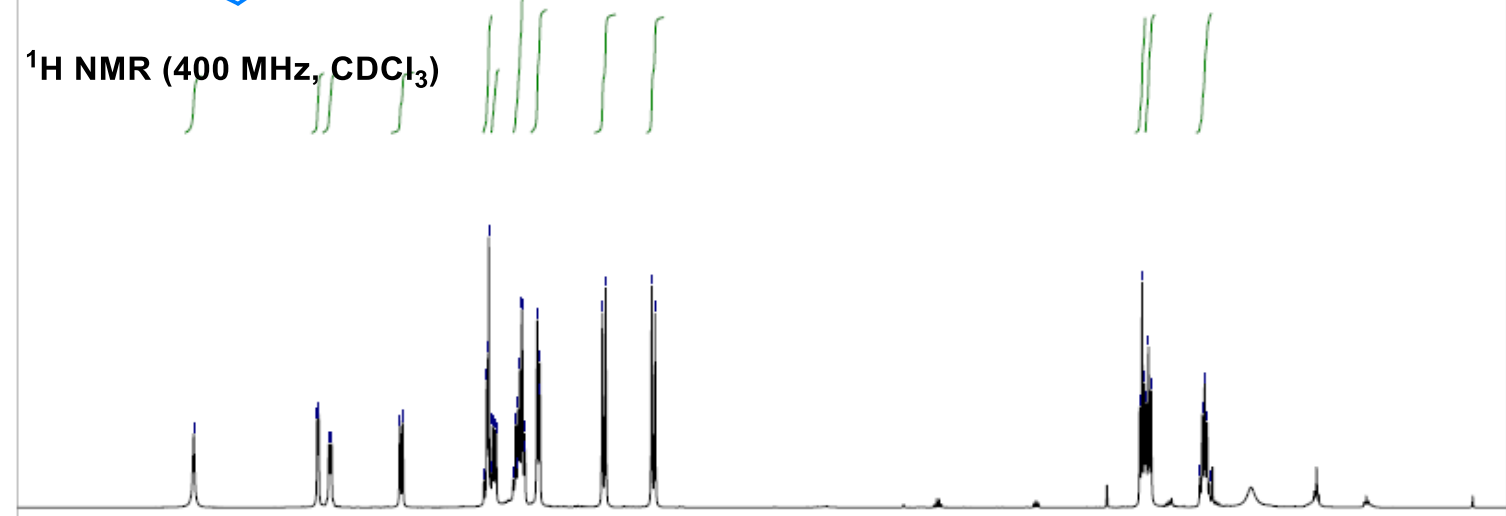

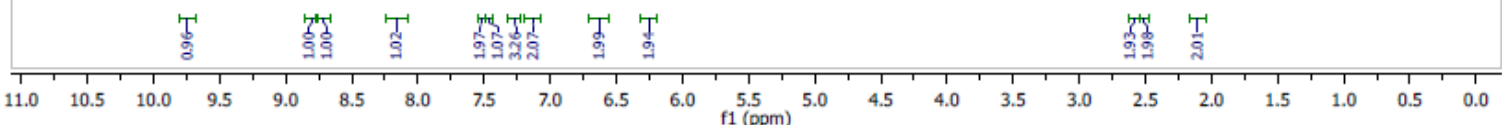

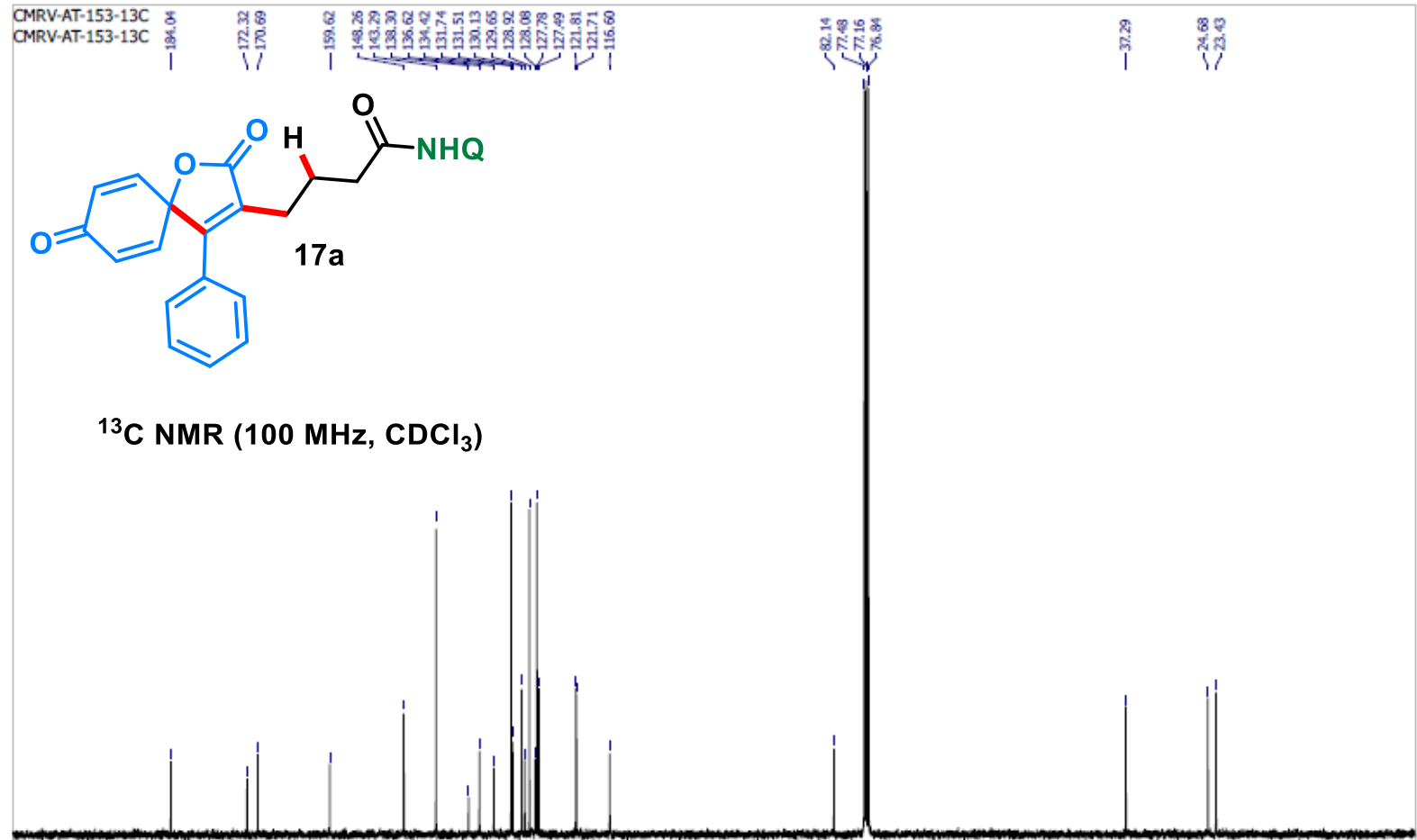

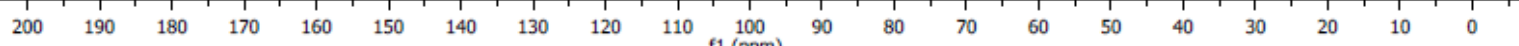



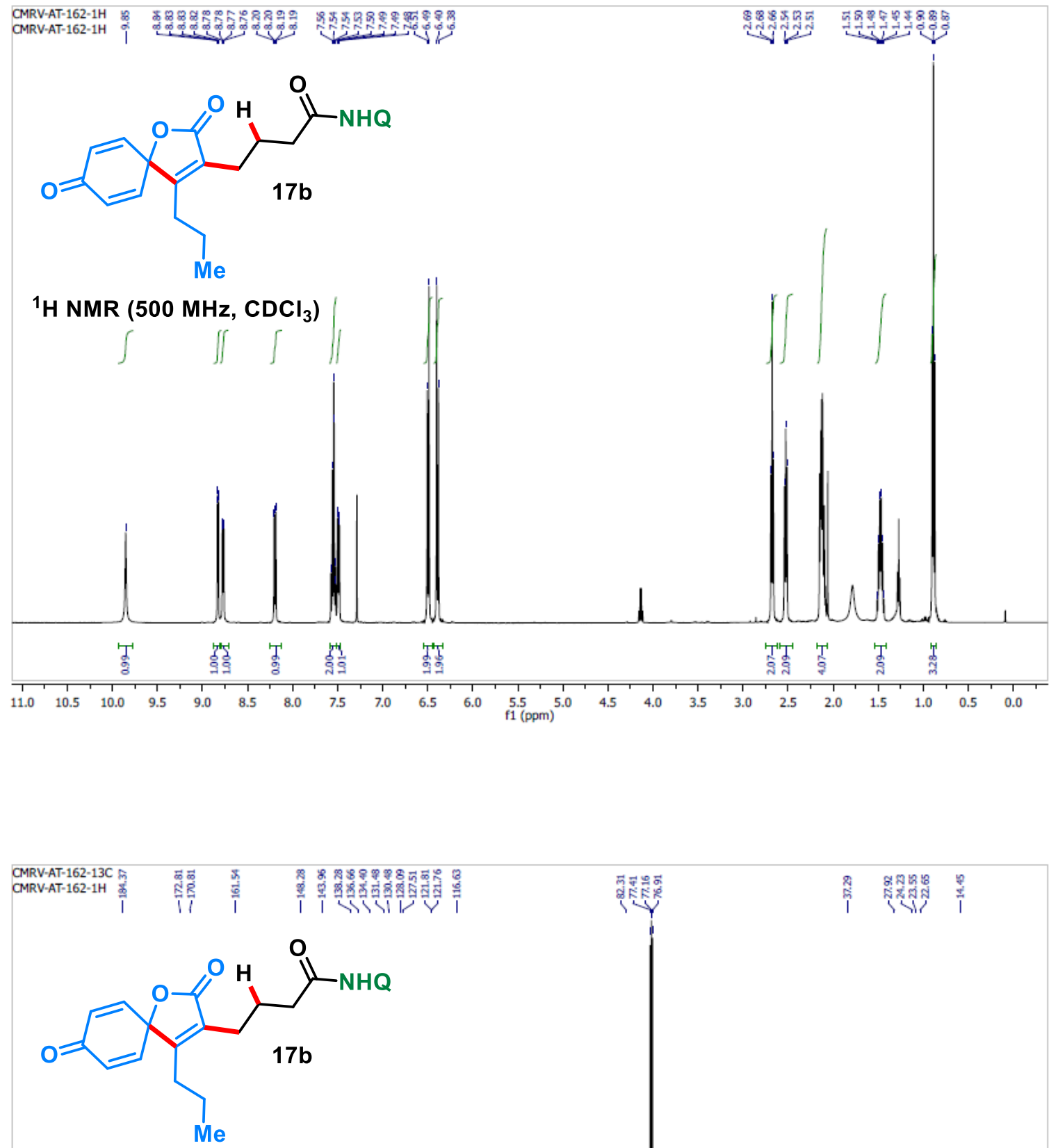

${ }^{13} \mathrm{C}$ NMR (125 MHz, $\mathrm{CDCl}_{3}$ )

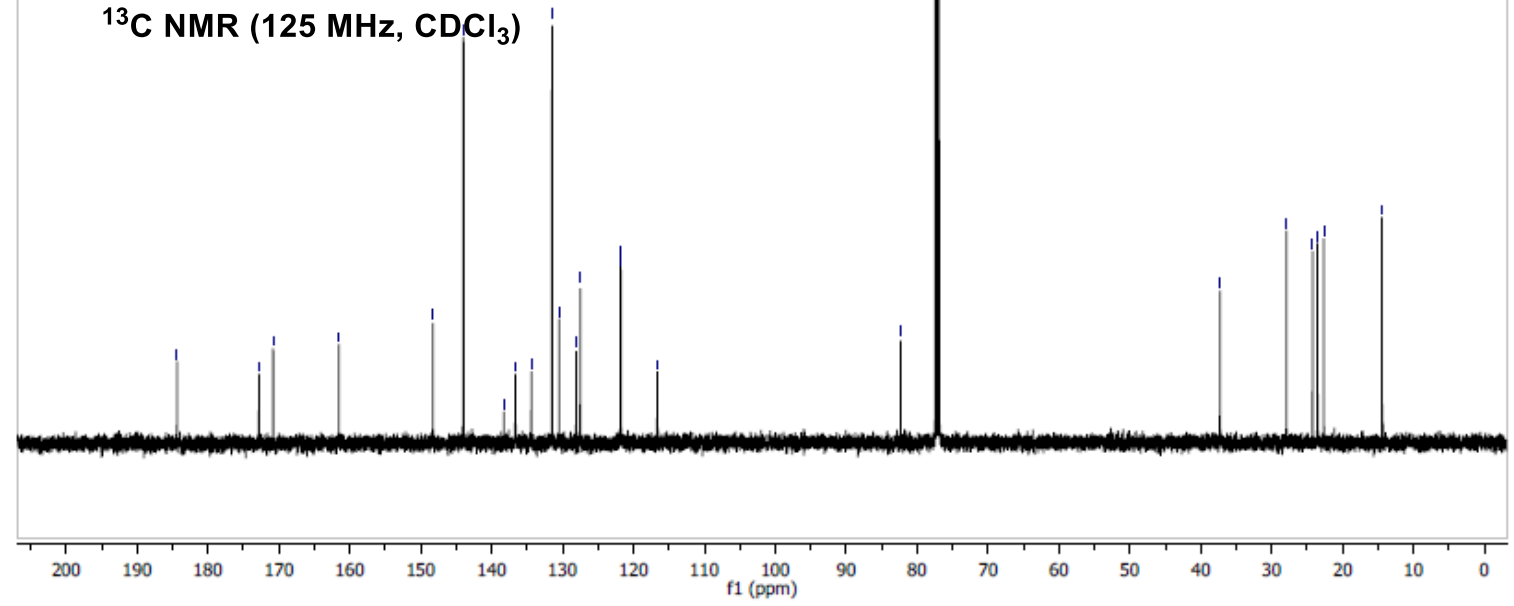




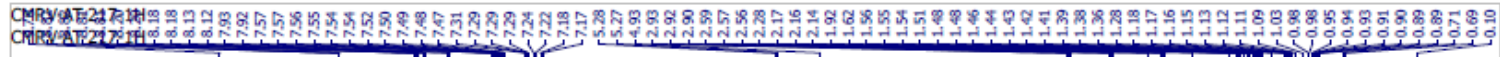
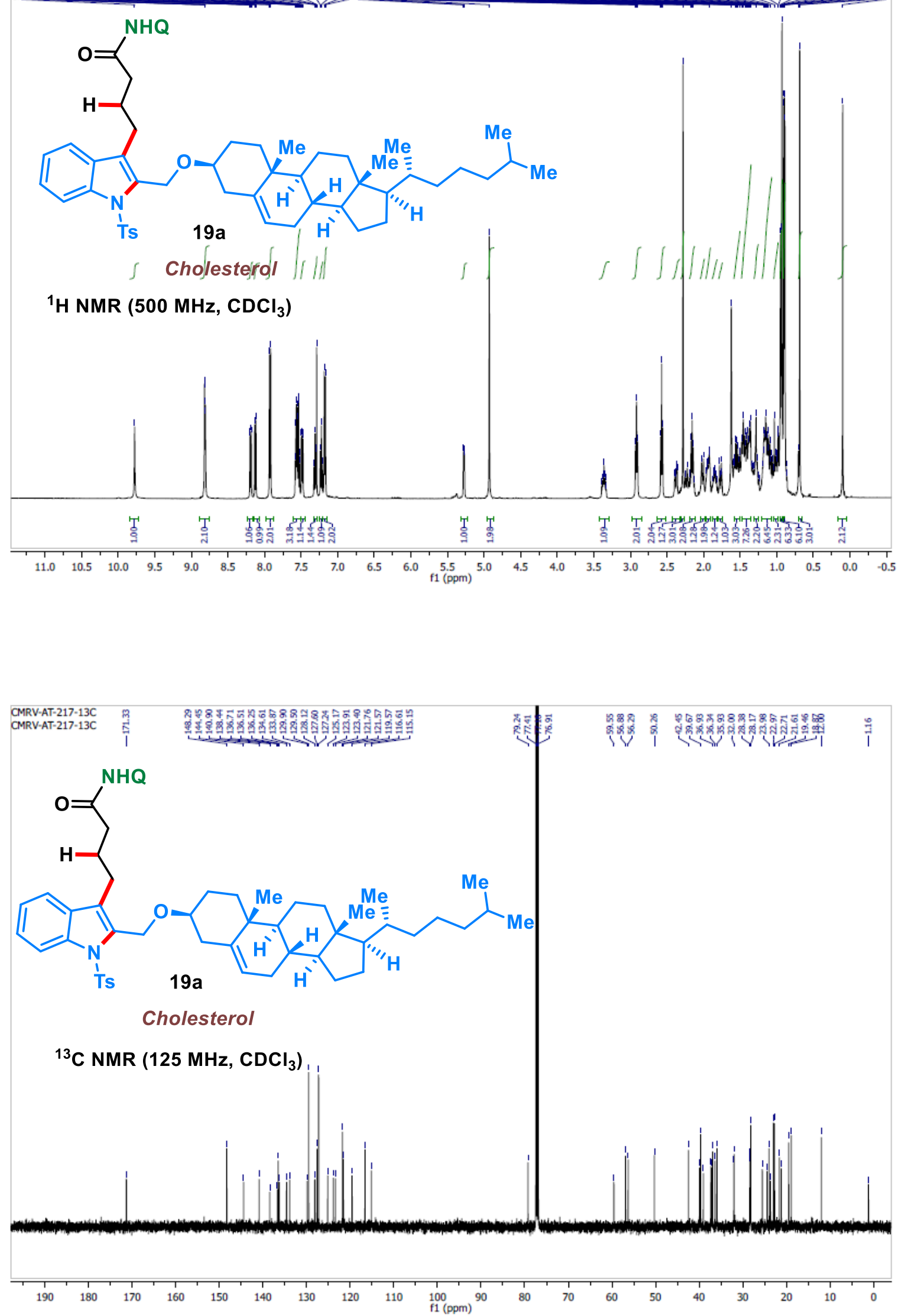


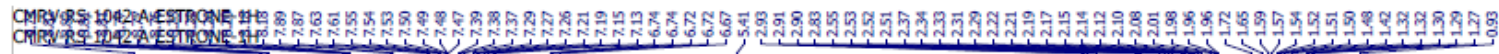
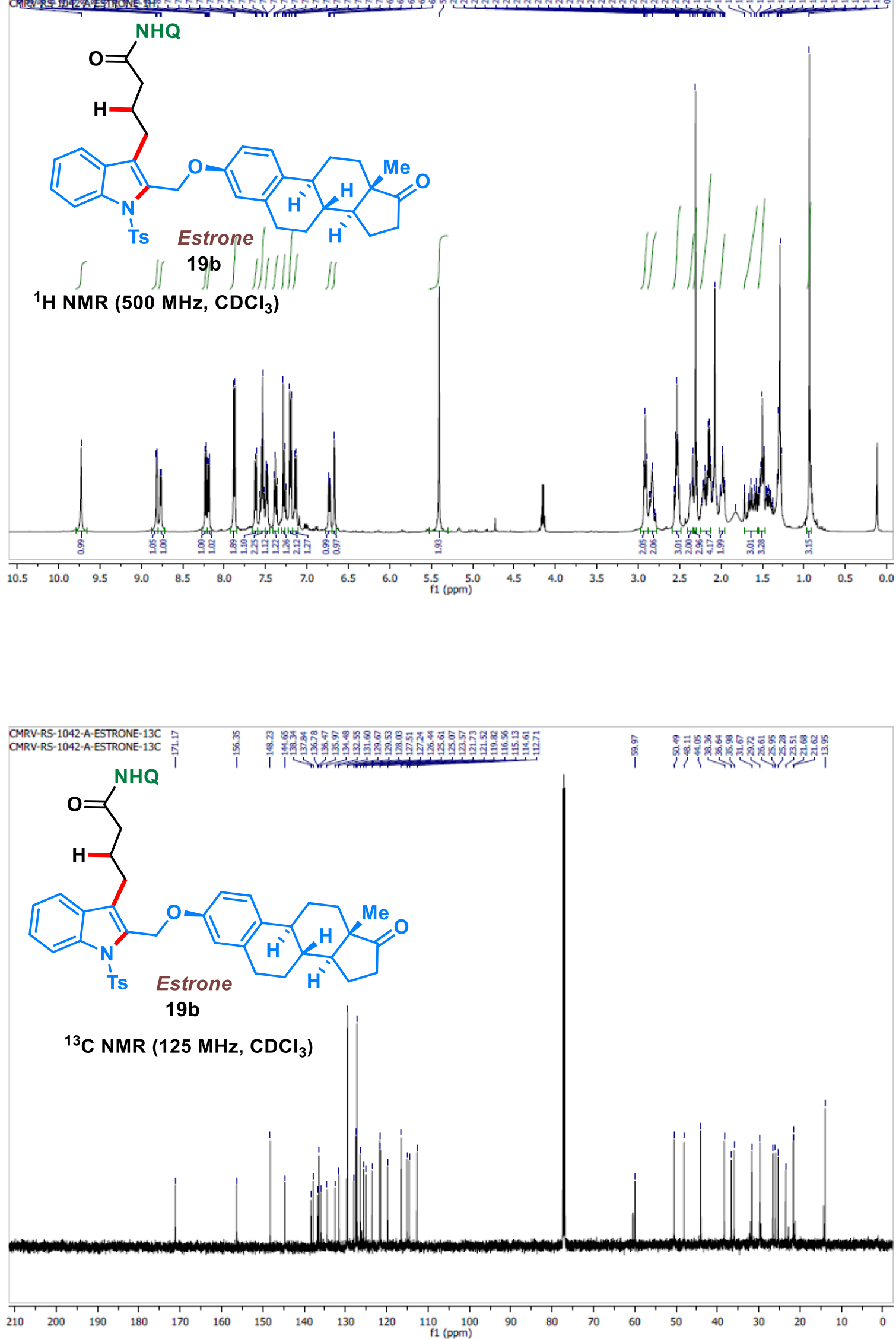


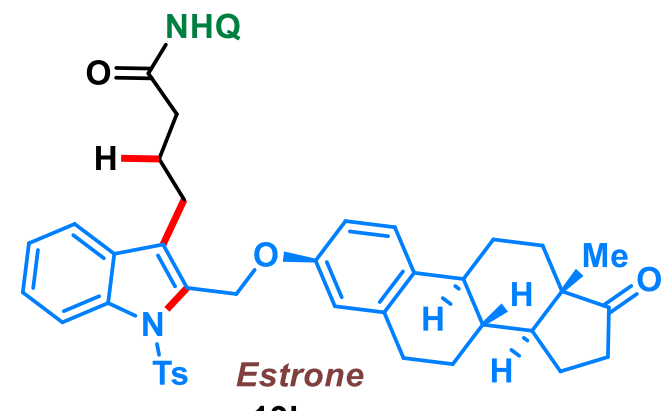

19b

${ }^{13} \mathrm{C}$ NMR (125 MHz, CDCl $)$

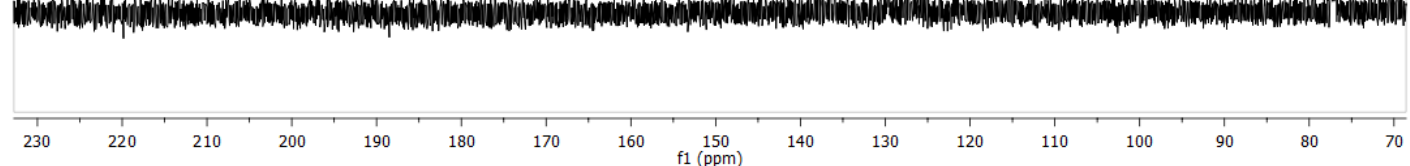




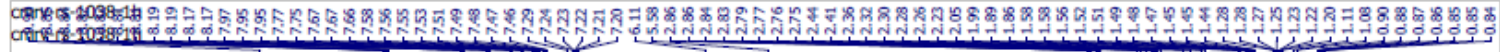

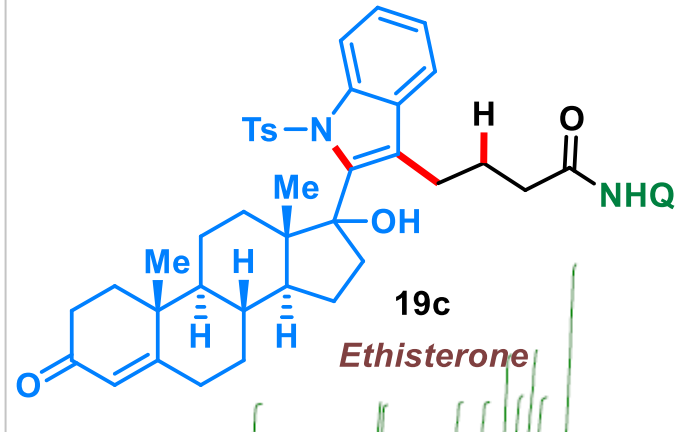

${ }^{1} \mathrm{H}$ NMR $\left(500 \mathrm{MHz}, \mathrm{CDCl}_{3}\right)$
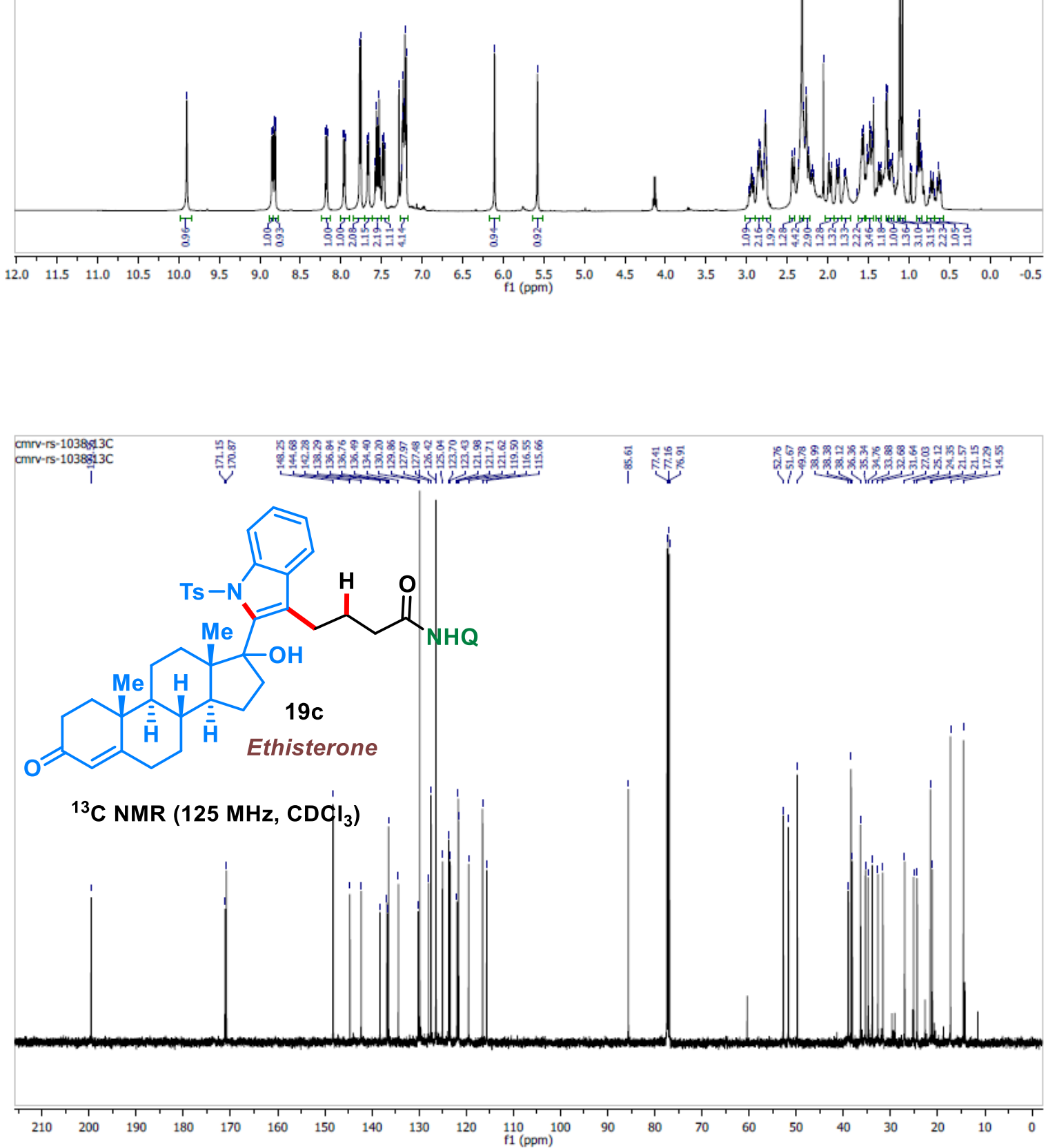

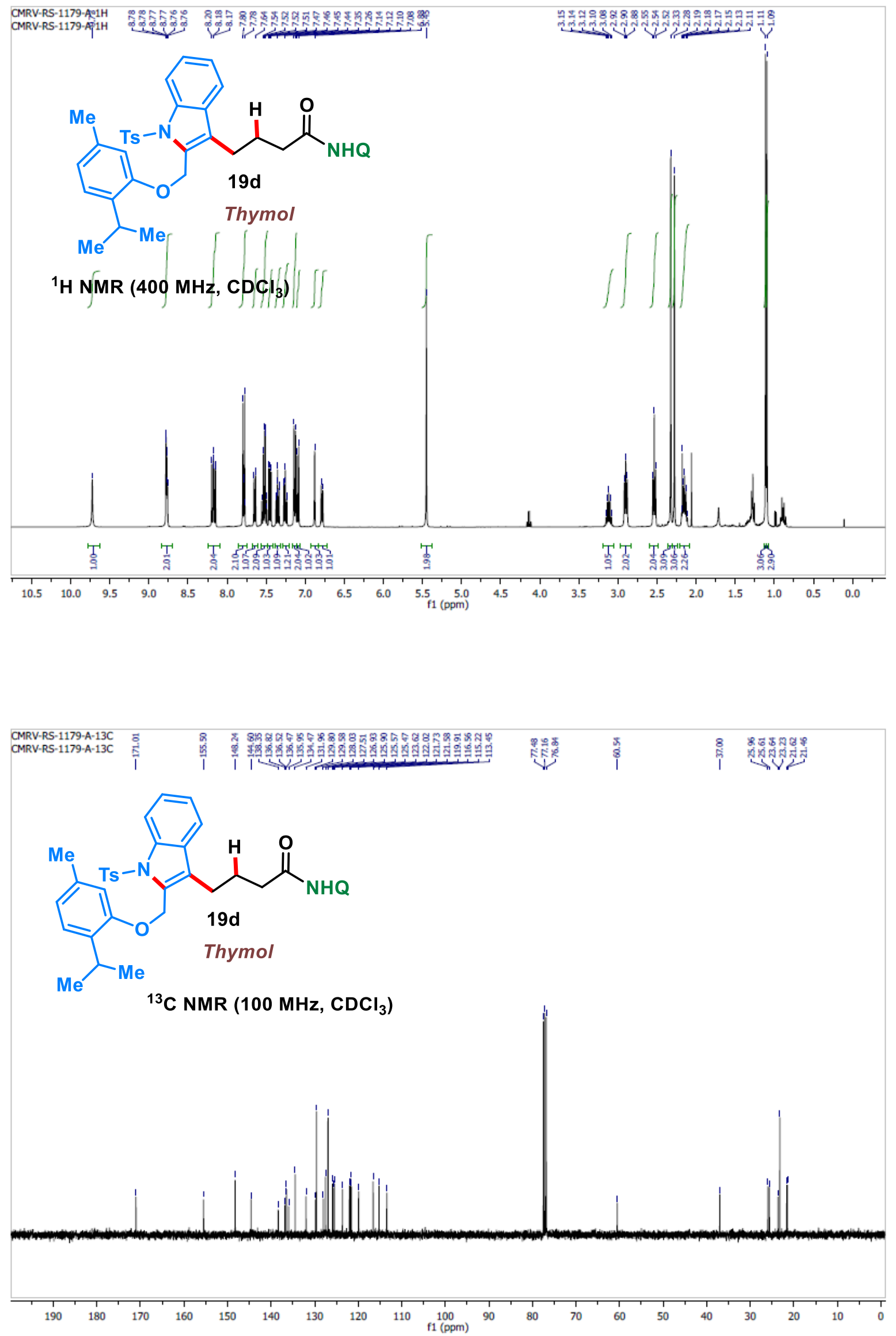

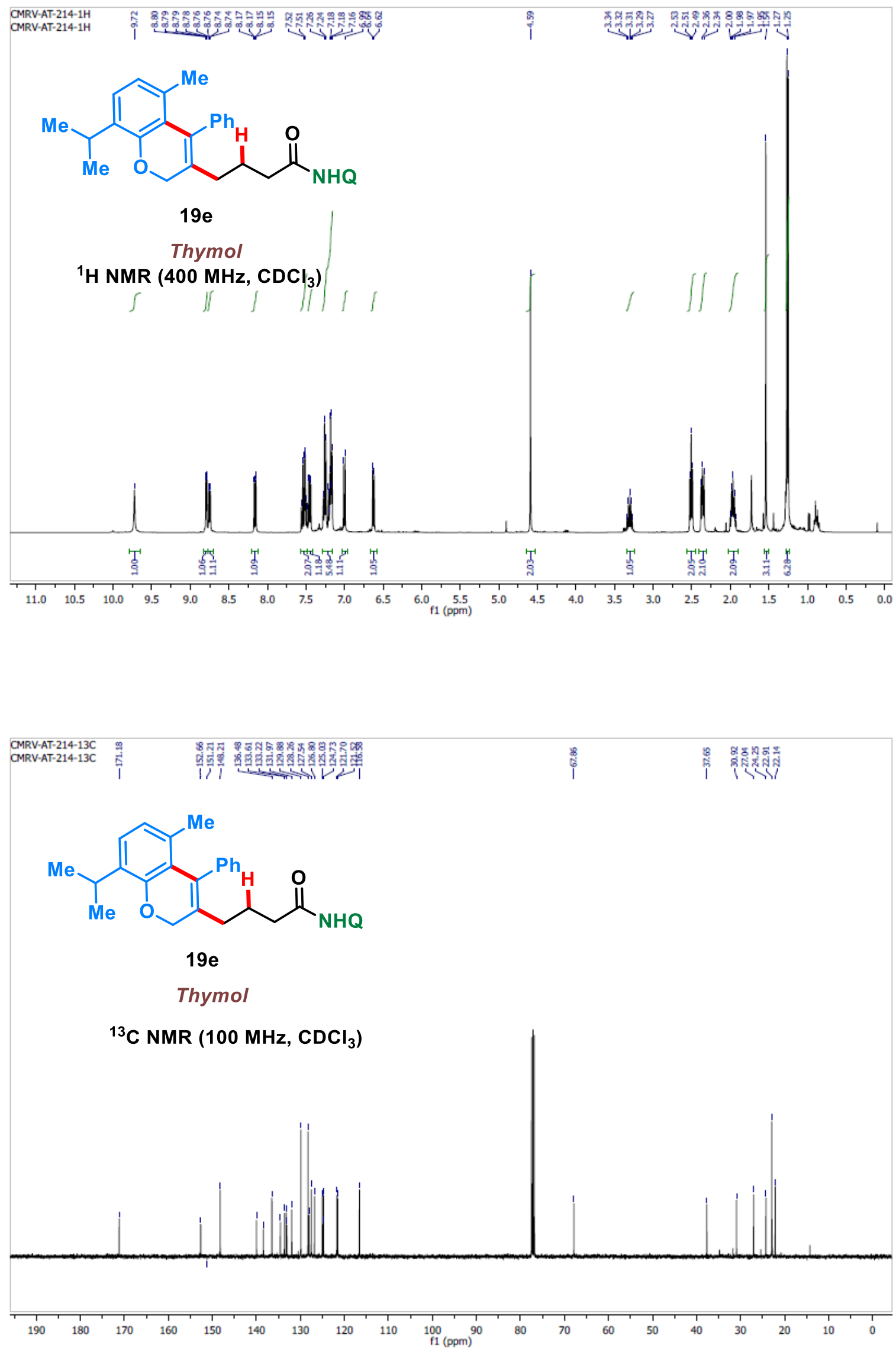

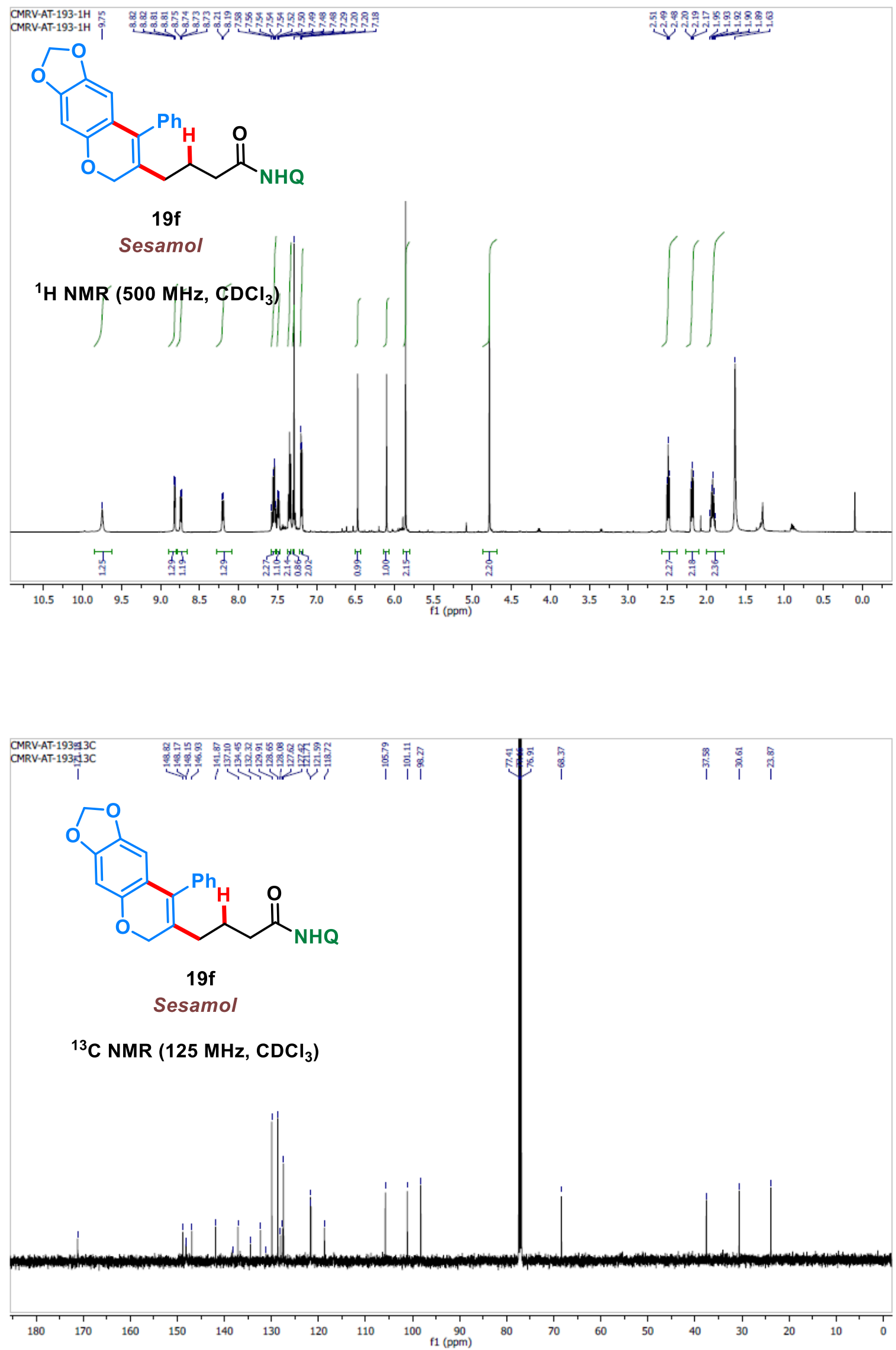


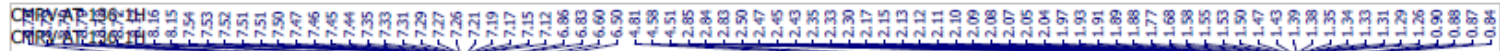
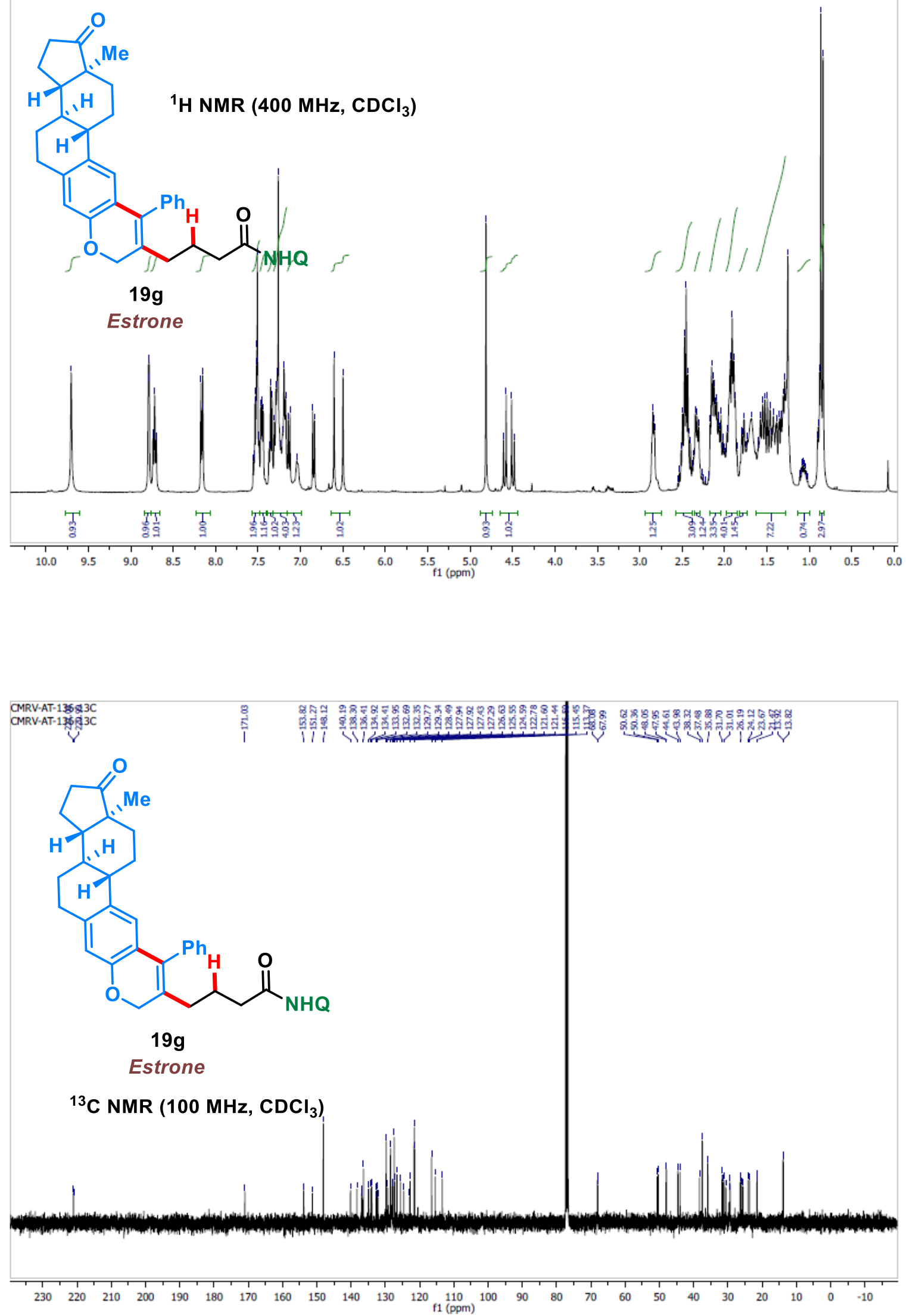

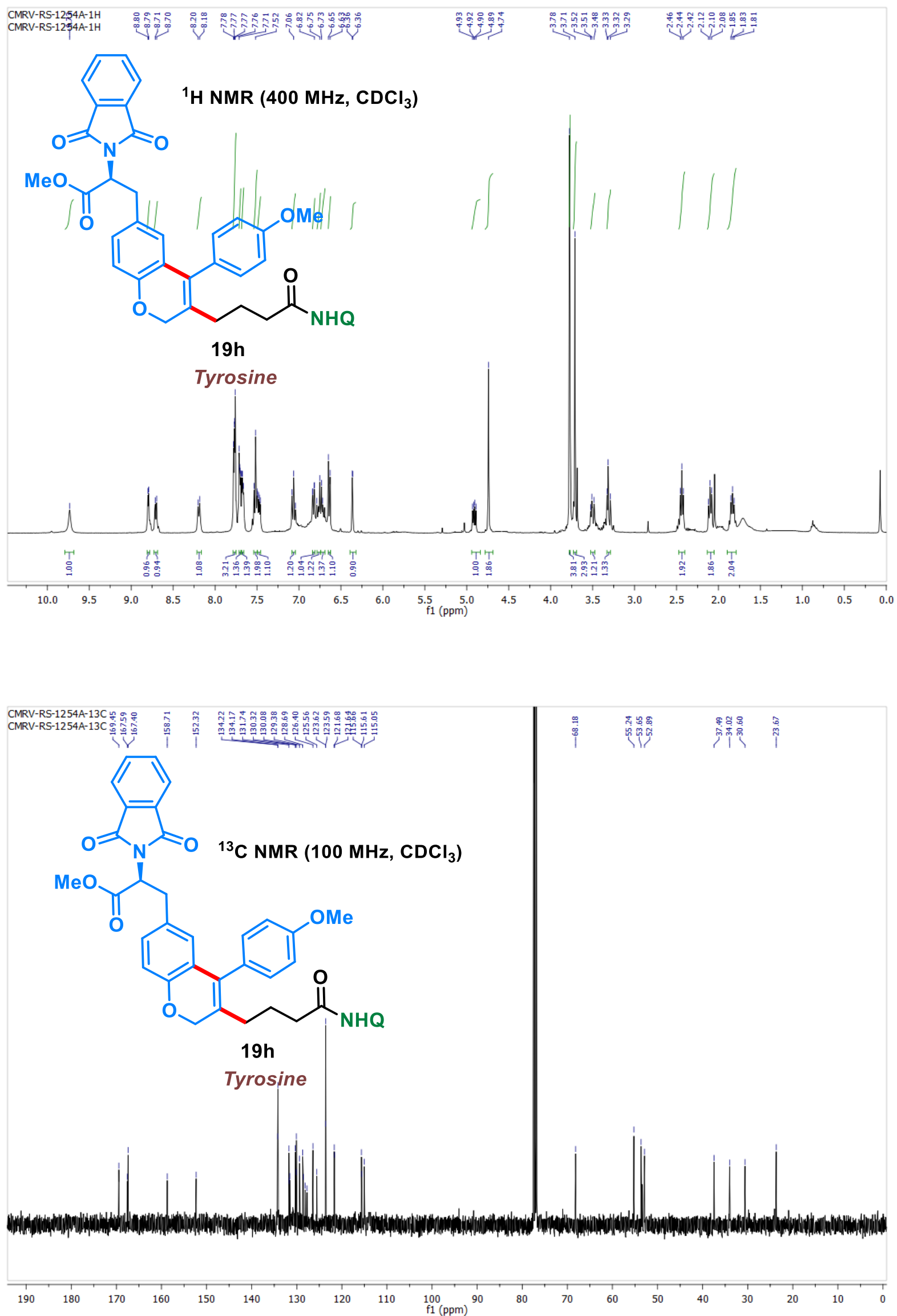

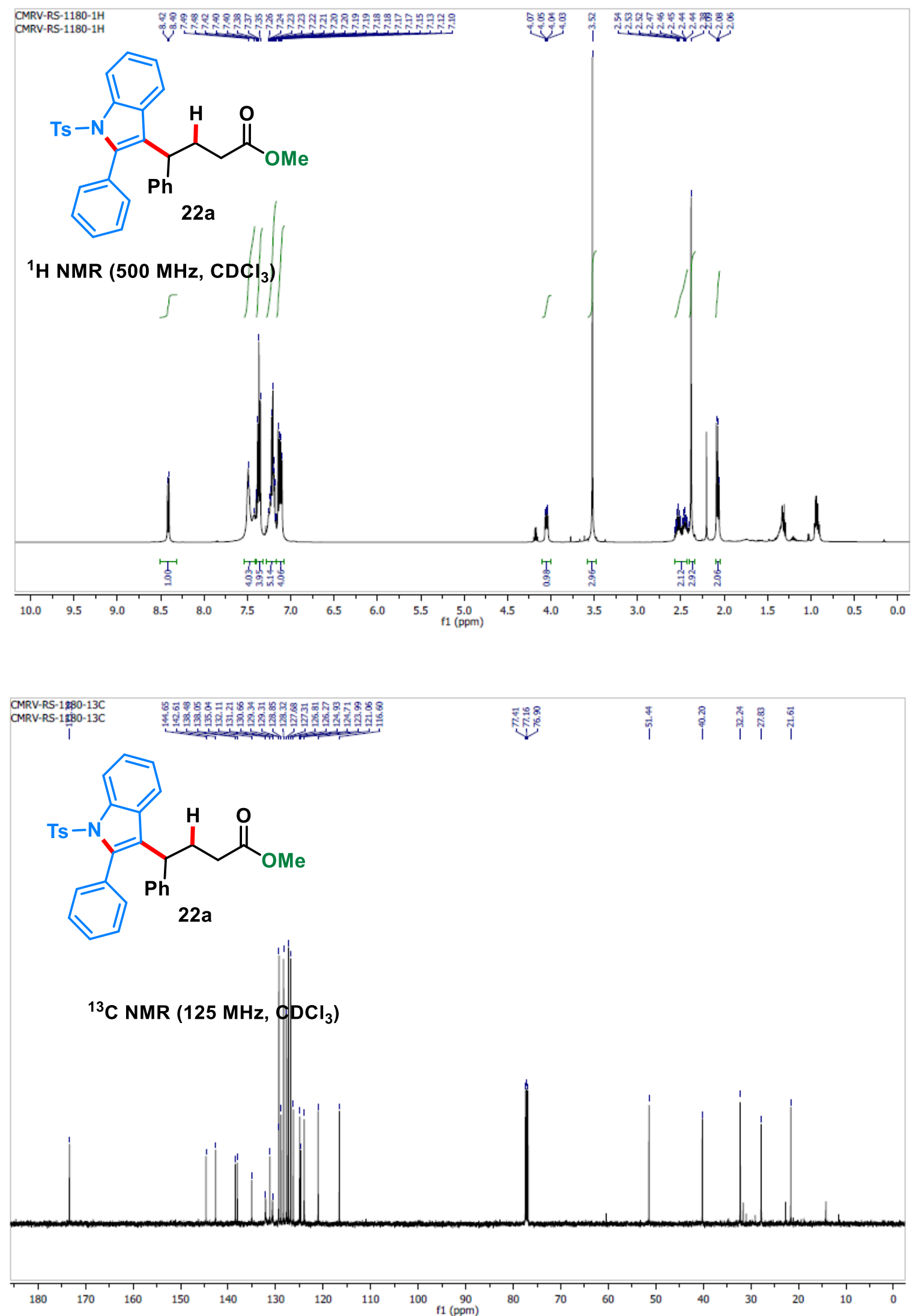

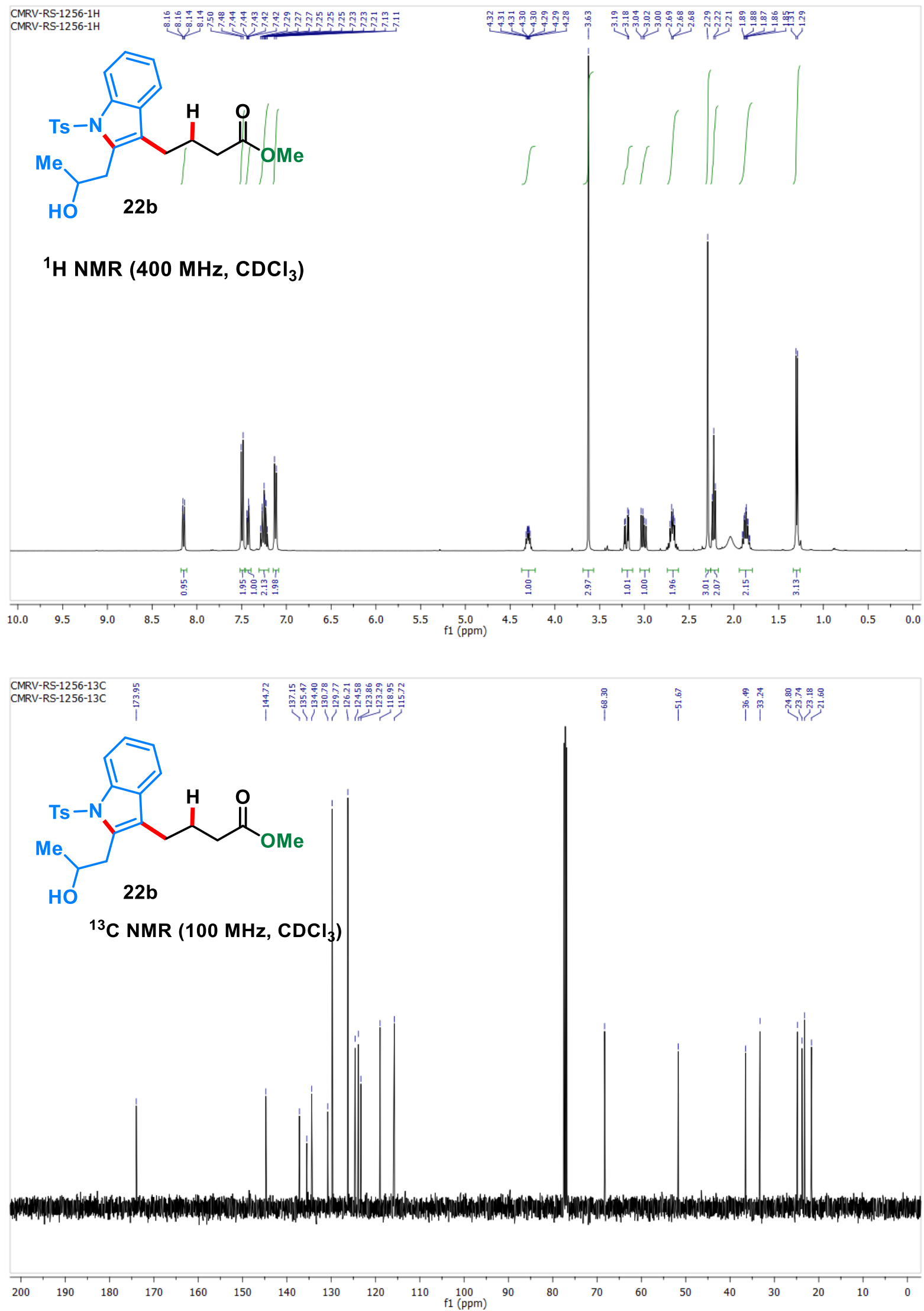

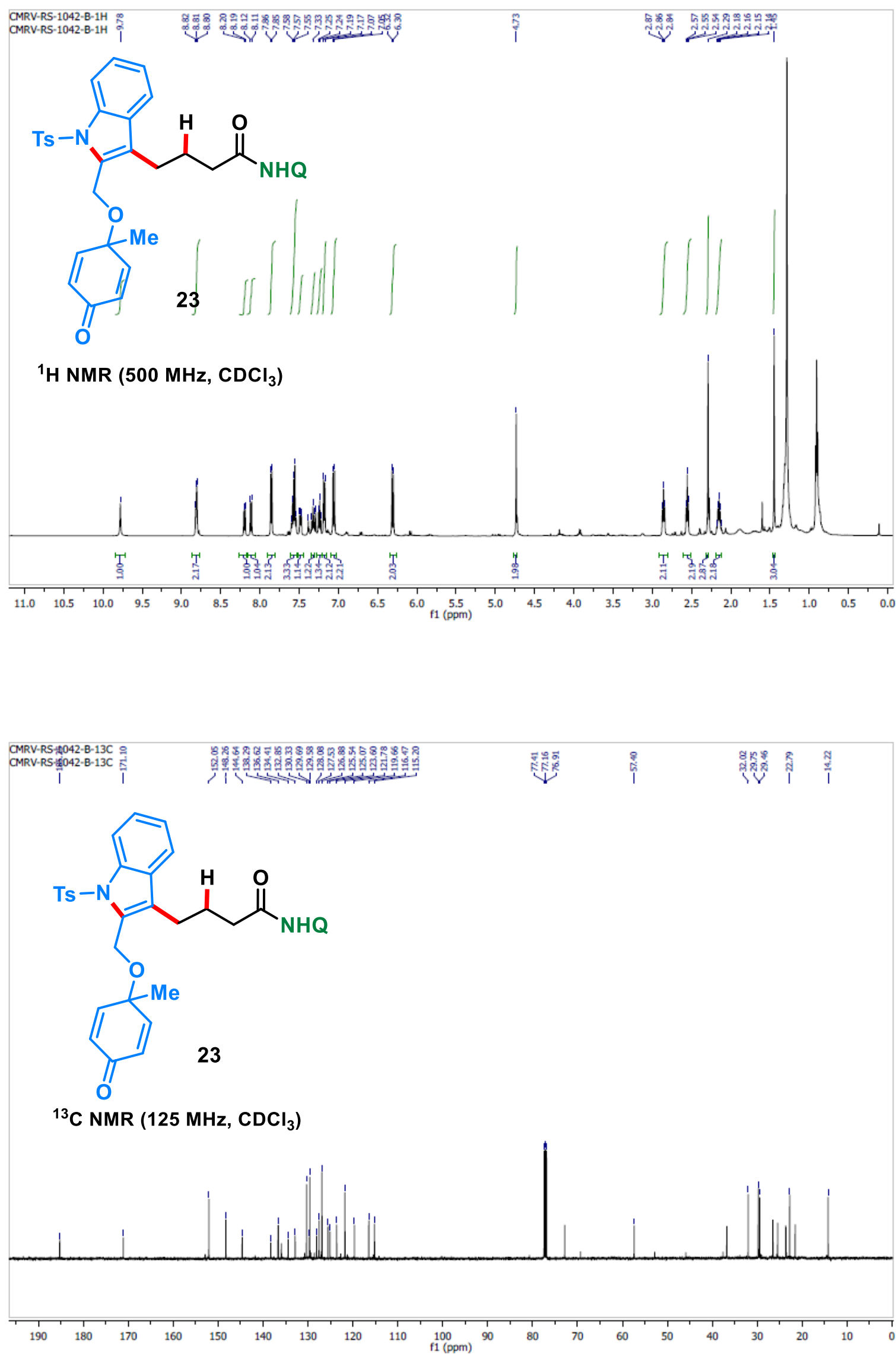

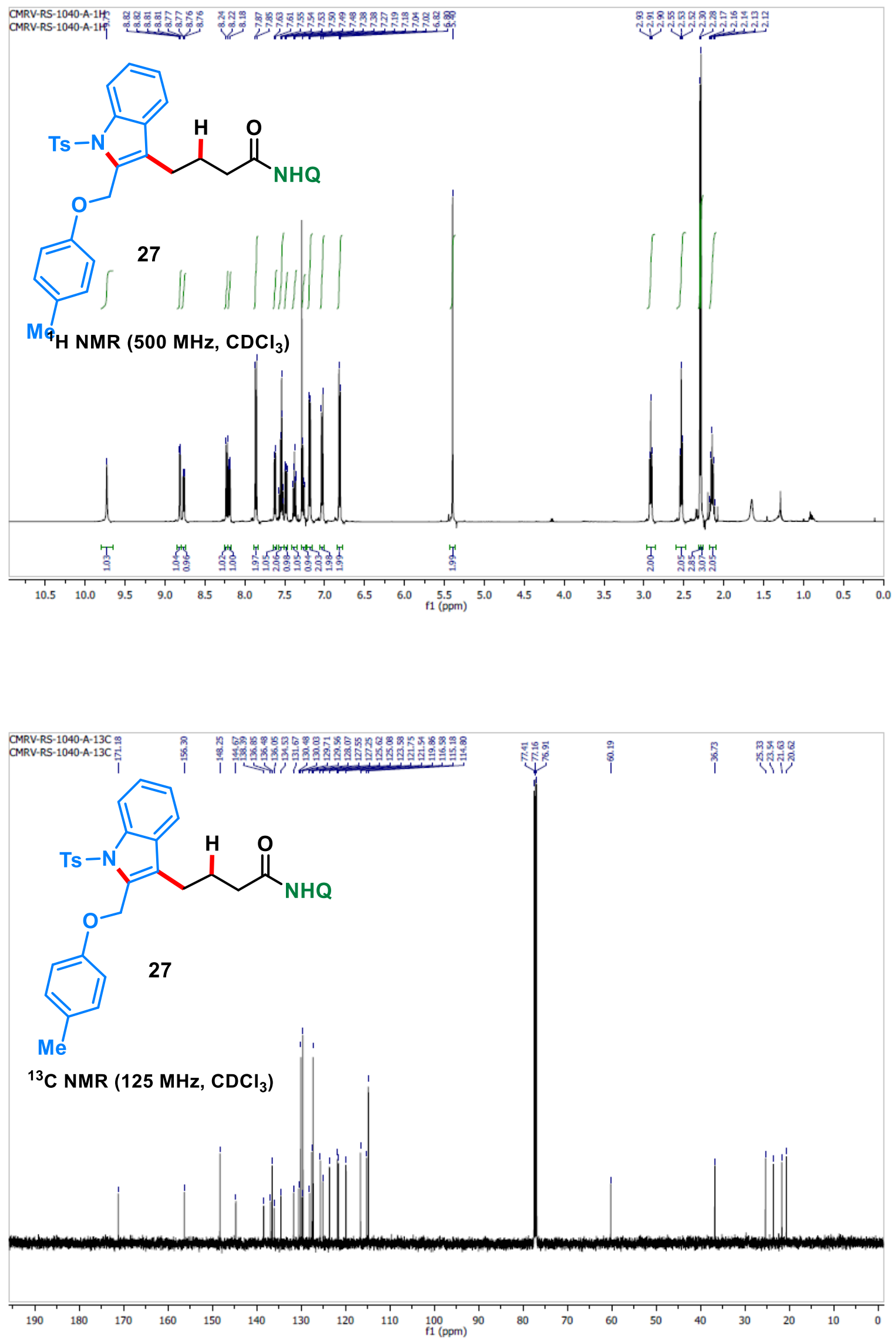


\section{DEPARTMENT OF CHEMISTRY, I.I.T.(B)}

Analysis Info

Analysis Name

Method

Sample Name

Comment
D:IDatalMAY 2021lcmrv-rs-pds2.d

Naformat_pos_1000a.m

cmrv-rs-pds2

C34H28N303PdS+
Acquisition Date $\quad 5 / 25 / 20218: 41: 37$ PM

Operator PG-SRD-OUT

Instrument maXis impact 282001.00081

\section{Acquisition Parameter}

Source Type ESI

Focus

Scan Begin

ESI

$50 \mathrm{~m} / \mathrm{z}$

$1000 \mathrm{~m} / \mathrm{z}$

Ion Polarity

Set Capillary

Set Corona

$2000 \mathrm{~V}$

Set Nebulizer

Set Divert Valve Source

\begin{tabular}{ccc} 
Set Corona & $\mathrm{OnA}$ & Set APCl Heater \\
\hline
\end{tabular}

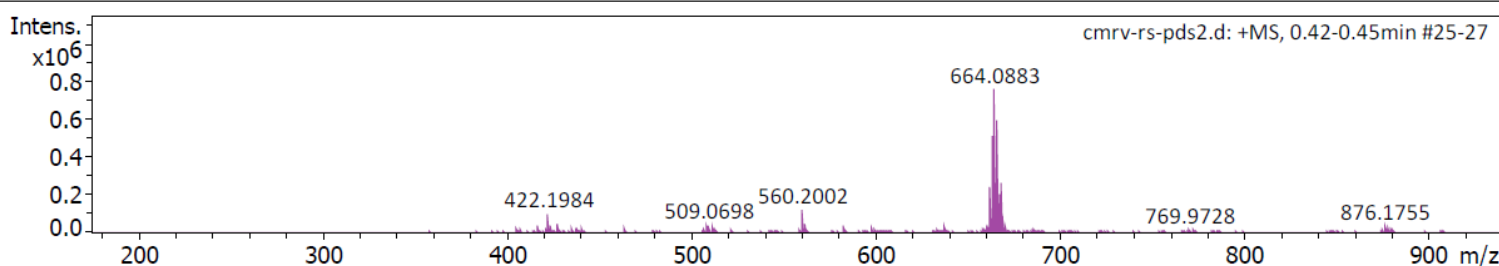

200300

$400 \quad 500 \quad 600$

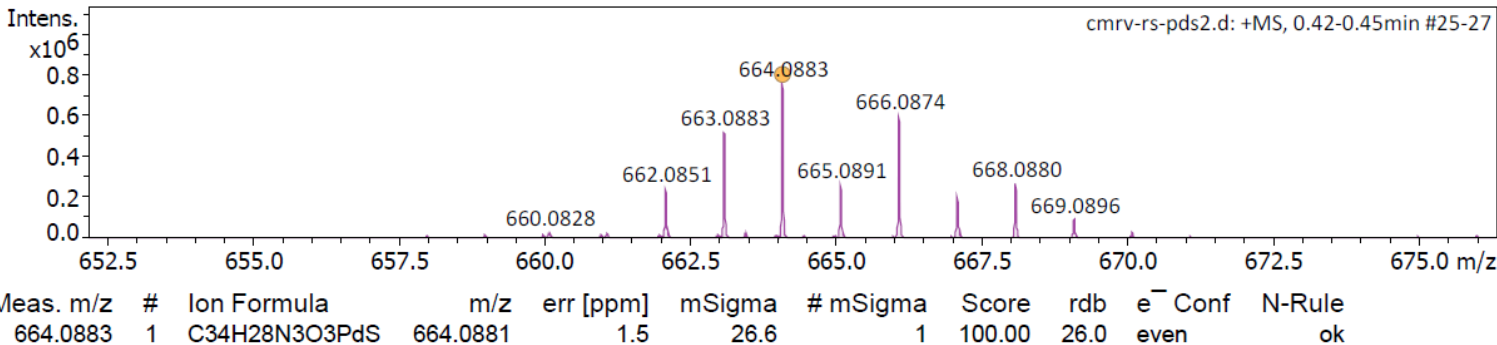

$\begin{array}{llllllllll}664.0883 & 1 & \mathrm{C} 34 \mathrm{H} 28 \mathrm{~N} 303 P d S & 664.0881 & 1.5 & 26.6 & 1 & 100.00 & 26.0 & \text { even }\end{array}$

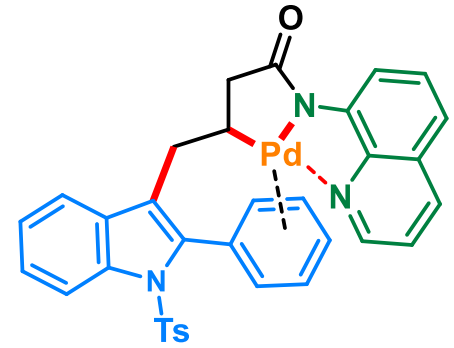

Chemical Formula: $\mathrm{C}_{35} \mathrm{H}_{30} \mathrm{~N}_{3} \mathrm{O}_{3} \mathrm{PdS}$

Pyridine was dissociated during measurement of HRMS, we got $\mathrm{M}+\mathrm{H}$ 